\title{
Nuclear models for exotic nuclei of relevance for astrophysics applications
}

\author{
S. Goriely \\ Institut d'Astronomie et d'Astrophysique - Brussels University
}


- Nuclear astrophysics needs

- the $r$ - and p-process nucleosynthesis

- Modelling nuclear reactions and the nuclear needs

- Statistical model of Hauser-Feshbach

- Pre-equilibrium model

- Direct capture

- Nuclear ingredients for reaction calculations

- Ground state properties

- $\gamma$-ray strength

- Nuclear Level Densities

- Optical Potential

- Fission

Conclusions: many more open questions than answers 


\section{Nuclear needs for nucleosynthesis applications}

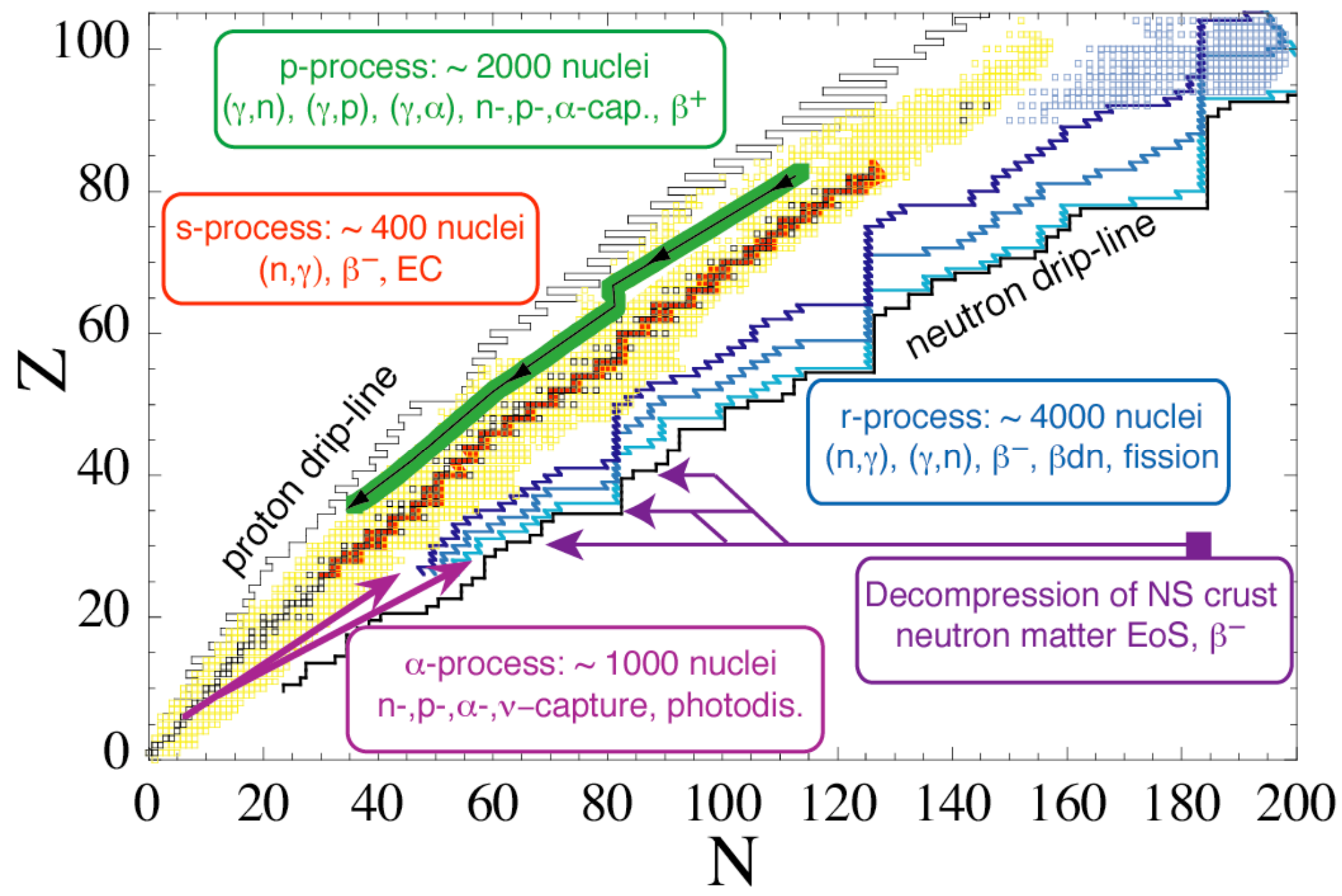

$\odot$ Large number of nuclei and properties involved; Exotic species

$\because$ Still large uncertainties in astrophysics modelling (hence nuclear needs !) 


\section{The r-process nucleosynthesis}

one of the still unsolved puzzles in astrophysics

... the r-process site remains unknown ...

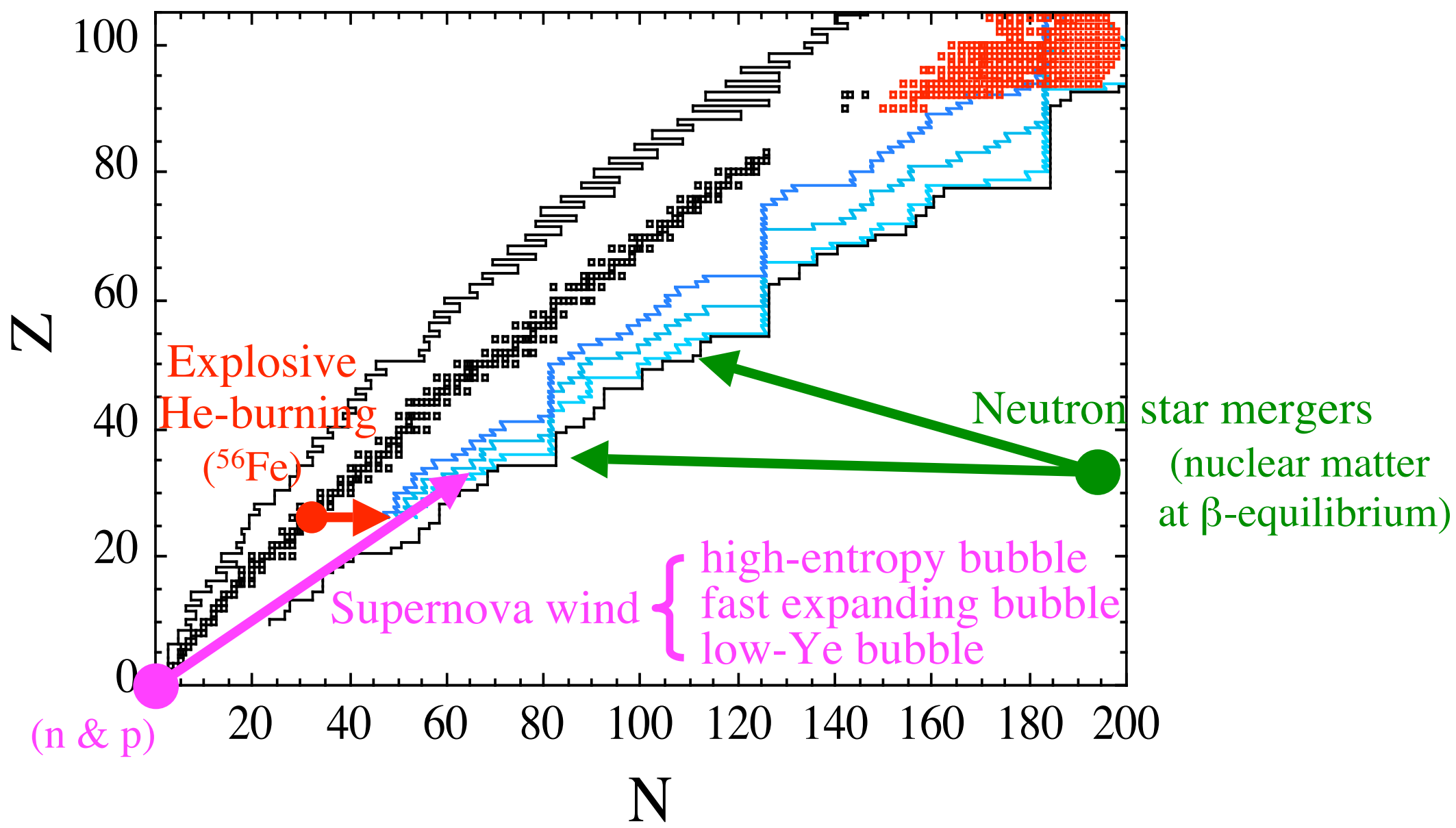

Many subjective interpretations, unconfirmed speculations, fast conclusions, ... 


\section{Possible origin of the r-process nuclei:}

$\underline{\text { Model }}$

"hot" $v$-driven wind

"cold" $v$-driven wind

Ext. inner crust of NS

Outer crust of NS $\underline{\text { Mechanisms }}$

$(n, \gamma)-(\gamma, n)$ equilibrium

n-capture and $\beta$-decays competition

nuclear and $\beta$-equil.

at non-zero $\mathrm{T}$ $\beta$-decays and "masses"

$+v$-nucleus interaction

$+(\mathrm{n}, \gamma)$ rates $(?)$

$\underline{\text { Nuclear needs }}$

$\beta$-decay $\&(\mathrm{n}, \gamma)$ rates $\& v$-int EoS of asymmetric NM

Masses, EoS, Coulomb

$\mathrm{EC}$ rates

Production by fission recycling (A 120-150 \& A>206)

Efficient $v$-driven wind Int. inner crust of NS
Fission products

Decay chains $\beta$-decay \& $(n, \gamma)$ rates fission rates \& FP distr. $\alpha$-decay - Spont. fission 


\section{Nuclear Physics associated with the r-process}

(Still large uncertainties in astrophysics modelling, hence nuclear needs !)

Competition between

- radiative neutron capture $(n, \gamma)$

- photo-neutron emission $(\gamma, \mathrm{n})$

- $\beta$-decay

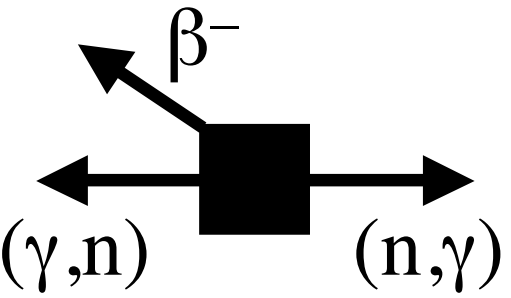

- fission (n-induced, $\beta$-delayed, spont.) for the heaviest species

- $v$-nucleus interaction properties

FOR POTENTIALLY ALL NUCLEI ( 5000) FROM THE VALLEY OF STABILITY TO THE NEUTRON DRIP LINE (not only for the so-called "waiting points")

We know these quantities will enter the problem but as long as the r-process site remains unknown, we cannot use astro simulation to judge

- quantitatively about the importance of a given ingredient, hence - even less about the quality of the nuclear input (from astro simulations) 


\section{P-process in Ne/O-rich layers during SNII explosion of massive stars}

1. s-process during core He-burning by ${ }^{22} \mathrm{Ne}(\alpha, n)^{25} \mathrm{Mg}$
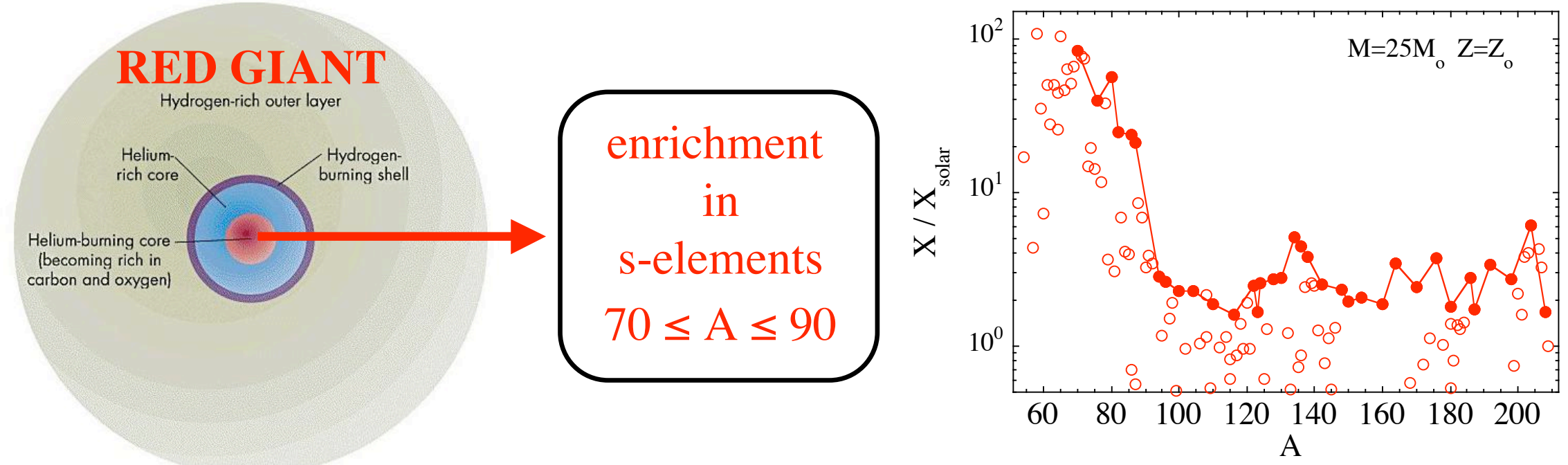

2. p-process in $\mathrm{O} / \mathrm{Ne}$ layers

(hydrostatic pre-supernova as well as explosive supernova phases)

Heating at $\mathrm{T}=2-310^{9} \mathrm{~K}$ of the s-enriched \& r-seeds $\left(\sim 0.7 \mathrm{M}_{\mathrm{o}}\right)$

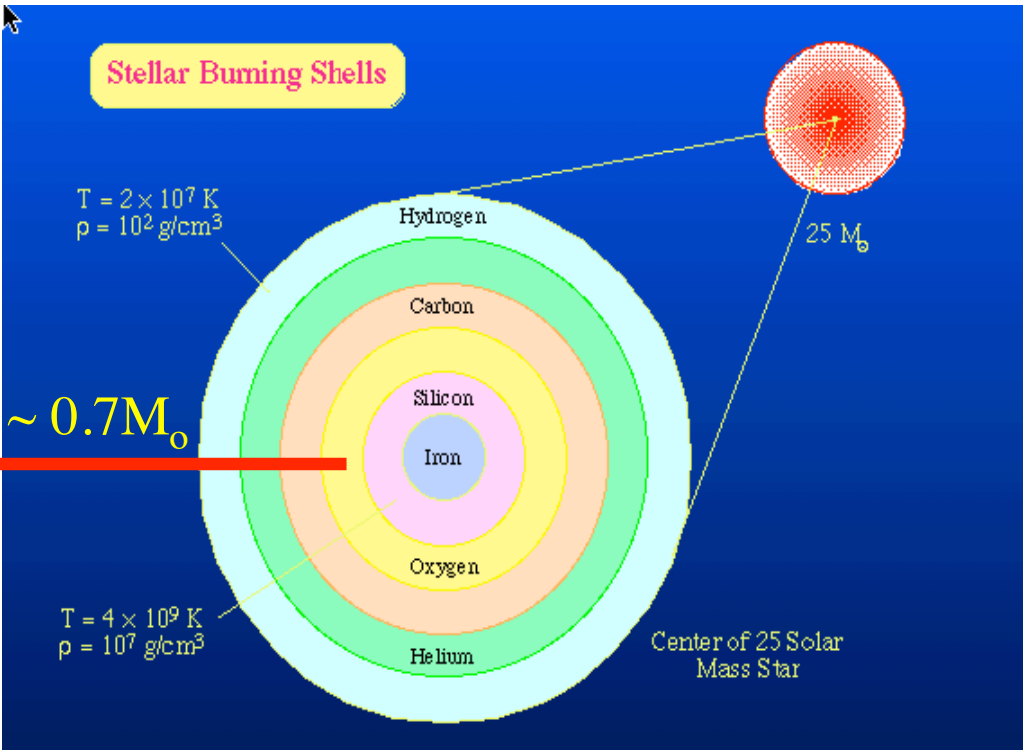




\section{Accreting White Dwarf models for type Ia Supernovae}

Matter accreted onto the surface of a White Dwarf (possibly enriched in selements during the AGB phase) from its binary companion causes regions in its interior to become unstable to thermonuclear runaway.

L

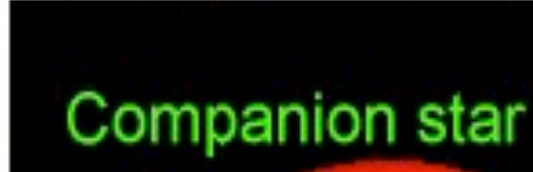

\section{Companion star}

Carbon deflagration and/or detonation (3D models available !!)

$\rightarrow$

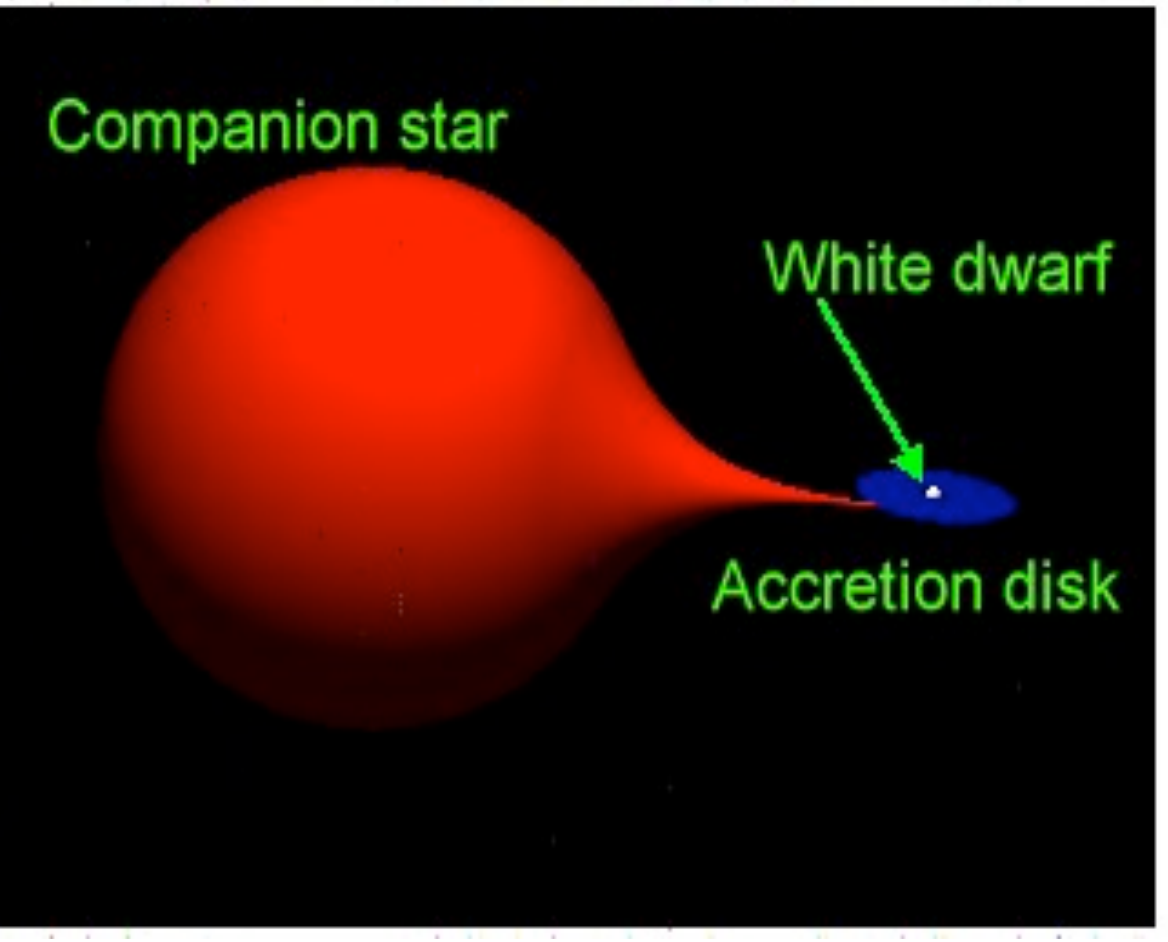

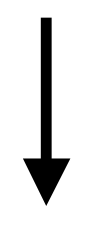

p-process nucleosynthesis in layers heated at $\mathrm{T}=2-310^{9} \mathrm{~K}$ (initial composition $\mathrm{C}+\mathrm{O}+\mathrm{Ne}$ ) 
A schematic representation of the p-processes

p-Process Reaction Path

Sn

In

Cd

Ag

Pd

$\mathrm{Rh}$

Ru

Tc

Mo 92

$\mathrm{Nb}$

95,96

$\mathrm{Zr} 9{ }_{90} 91,92-\square 94-\square 96$ 


\section{Nuclear needs for $\mathbf{p}$-process calculations}

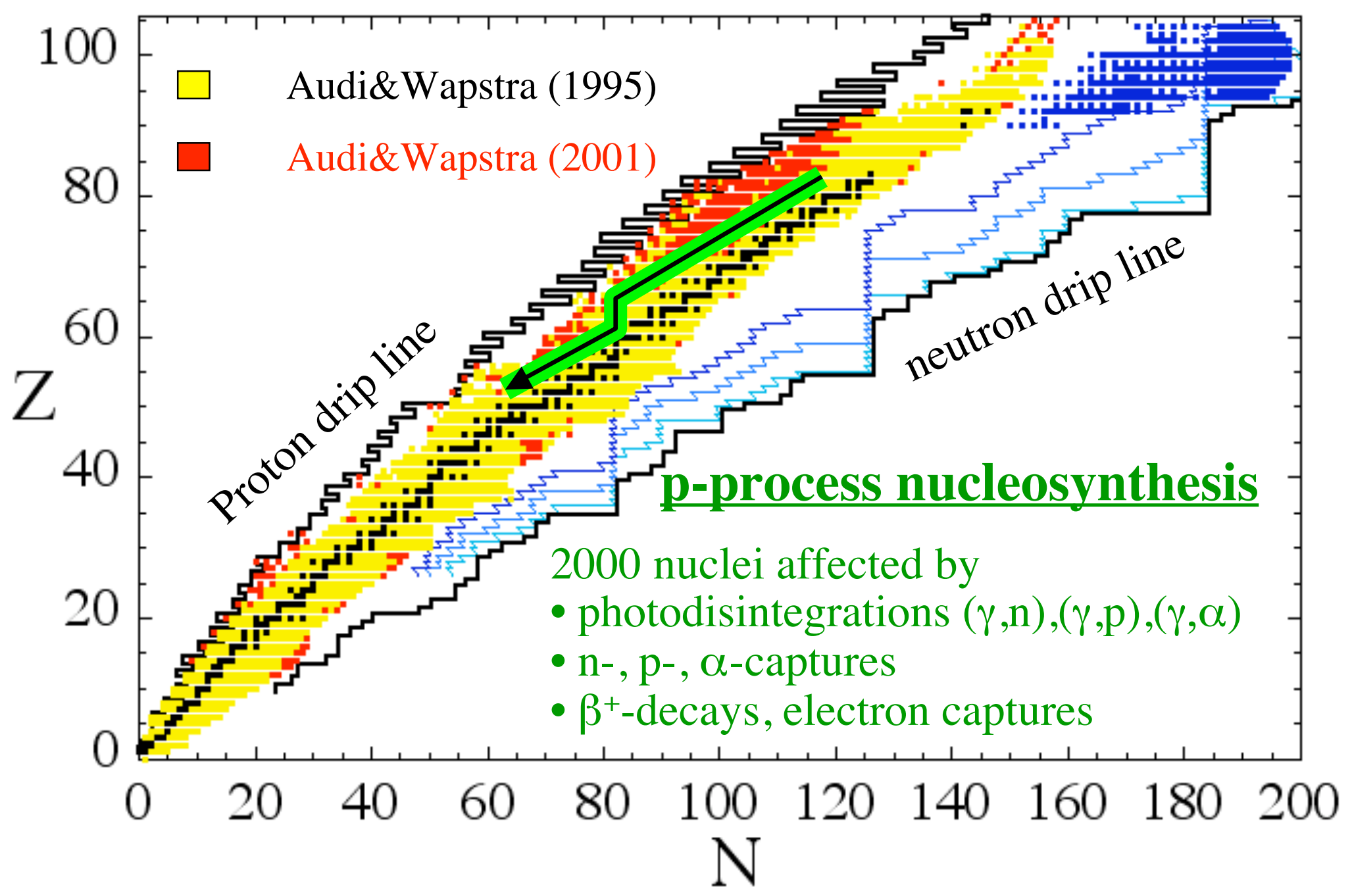

(-) Many nuclear masses and $\beta^{+}$-decay rates known experimentally

$\odot$ Almost no $\mathrm{p}$ - and $\alpha$-capture rates known experimentally 


\section{Impact of the nuclear uncertainties on the p-nuclide production}

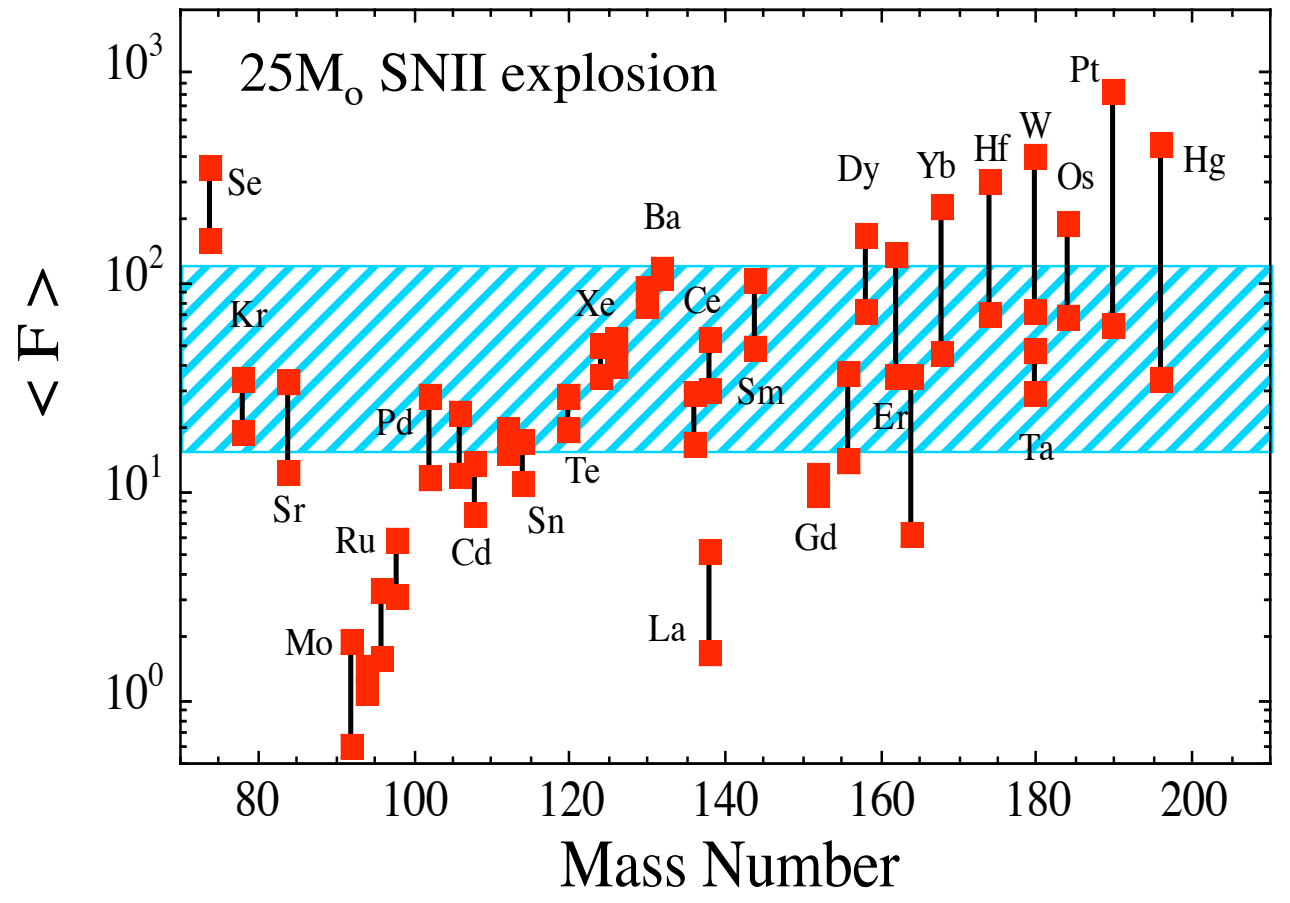

Major nuclear uncertainties from

- GLOBAL alpha-nucleus optical potentials (heavy A>150 p-nuclides)

- GLOBAL nucleon-nucleus potential, NLD, $\gamma$-strength (light $<90$ p-nuclides)

(The ${ }^{92,94} \mathrm{Mo},{ }^{96,98} \mathrm{Ru}$ discrepancies are most probably not related to nuclear issues)

- p- and $\alpha$-captures: new measurements (Demokritos, Debrecen, Kalrsruhe, ...) but still not enough contraints on global potential -

- $\gamma$-ray strengths: new experimental information (Konan, Oslo, Duke, GSI, ...), but still open debate on the low-energy tail and extra dipole strength at low energies 
Reactions of interest in nucleosynthesis applications

\section{Strong interaction}

- n-captures: $(n, \gamma),(n, p),(n, \alpha)$

- p-captures: $(p, \gamma),(p, n),(p, \alpha)$

- $\alpha$-captures: $(\alpha, \gamma),(\alpha, n),(\alpha, p)$

- fission: (n,f)

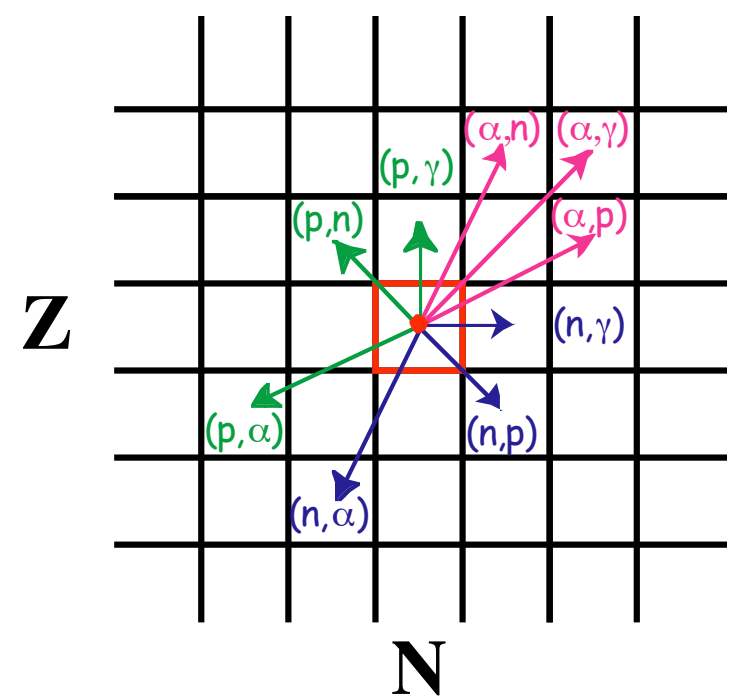

\section{Photodisintegrations}

$(\gamma, \mathbf{n}),(\gamma, \mathbf{p}),(\gamma, \alpha),(\gamma, \mathbf{f})$

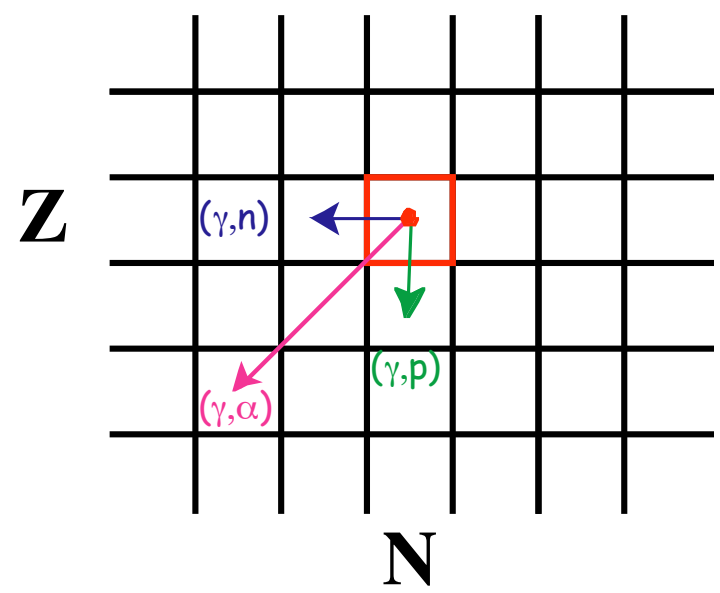

\section{Weak interaction}

$\beta$-decays: $\beta^{-}, \beta \mathrm{dn}, \beta^{+}, \mathrm{EC}, \beta \mathrm{dff}$ $v$-captures:

$$
\begin{aligned}
& (Z, A)+v_{e} \rightarrow(Z+1, A)^{*}+e^{-} \\
& (Z, A)+\bar{v}_{e} \rightarrow(Z-1, A)^{*}+e^{+} \\
& (Z, A)+v_{i} \rightarrow(Z, A)^{*}+v_{i}
\end{aligned}
$$

+ possible $\mathrm{n}^{-}, \mathrm{p}-\mathrm{,}, \alpha$-emission

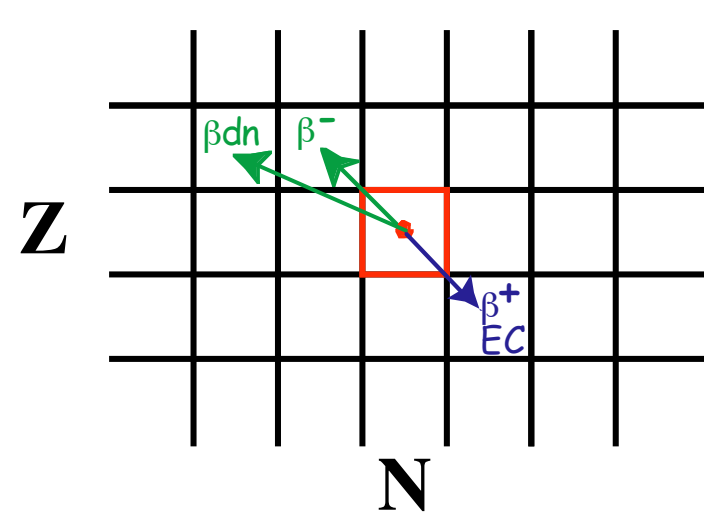

for $\sim 8000$ nuclei ranging from $H$ to $Z=110$ (?) lying between the $p$ - and $n$-driplines 
Astrophysics needs for nuclear data are defined by the sensitivity

of the astrophysics predictions to the nuclear inputs

Different types of astrophysics models

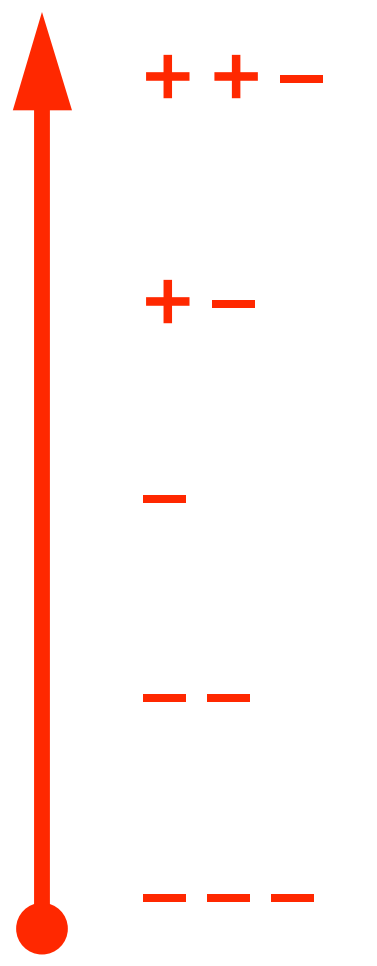

State of the art: 3D ( $\sim$ self-consistent $)$ models

p-process in SNIa explosions

Realistic 1D ( self-consistent) models

p- and s-processes in Massive Stars

Parametrized (semi-realistic) 1D models

s-process in AGB Stars, r-process in NSM

Parametrized (unrealistic) 1D models

$r$-process in v-driven wind

Phenomenological parametrized site independent models

Canonical s- and r-processes

No astrophysical model is free from uncertainties !

Obvious need for accurate and reliable nuclear data, ...

But the uncertainties in the astrophysics models most of the time prevail Astrophysics models cannot be used to extract nuclear properties for exotic nuclei ! 


\section{Reaction mechanisms}

In general terms, reactions mechanisms are divided in two major components:

- peripheral collision corresponding to the so-called direct contribution

- head-on collision corresponding to the so-called compound nucleus contribution

\section{DIRECT REACTIONS}

The projectile interacts primarily with nucleons at nuclear surface energy and mass transfer are small

Masses and energies of outgoing particles related to initial ones

Single-step process:

$$
a+A \rightarrow b+B
$$

Timescales $\sim 10^{-22} \mathrm{~s}$ (transit time through target nucleus)

Typical reactions: elastic and inelastic scattering transfer reactions (e.g. stripping, pick-up,...)

Processes more likely at high energies

$\lambda \sim 1 / E \Rightarrow \lambda$ decreases

$\Rightarrow$ interaction more likely with single nucleon

\section{COMPOUND NUCLEUS REACTION}

Incident particle has impact parameter $b<R$ (nuclear size) $\Rightarrow$ many nucleon-nucleon collisions inside composite system

= compound nucleus

Two-step process:

$$
\text { 1) } a+A \rightarrow C^{*} \quad \text { (formation of } C N \text { ) }
$$$$
\text { 2) } C^{*} \rightarrow b+B \quad(\text { decay of } \mathrm{CN} \text { ) }
$$

Initial energy of projectile share among many nucleons If (after many collisions) nucleon(s) acquire enough energy $\Rightarrow$ particles can be emitted $=$ PARTICLE EVAPORATION

Timescales $\sim 10^{-16}--10^{-18} \mathrm{~s}$ 
Direct reaction versus Compound Nucleus reaction

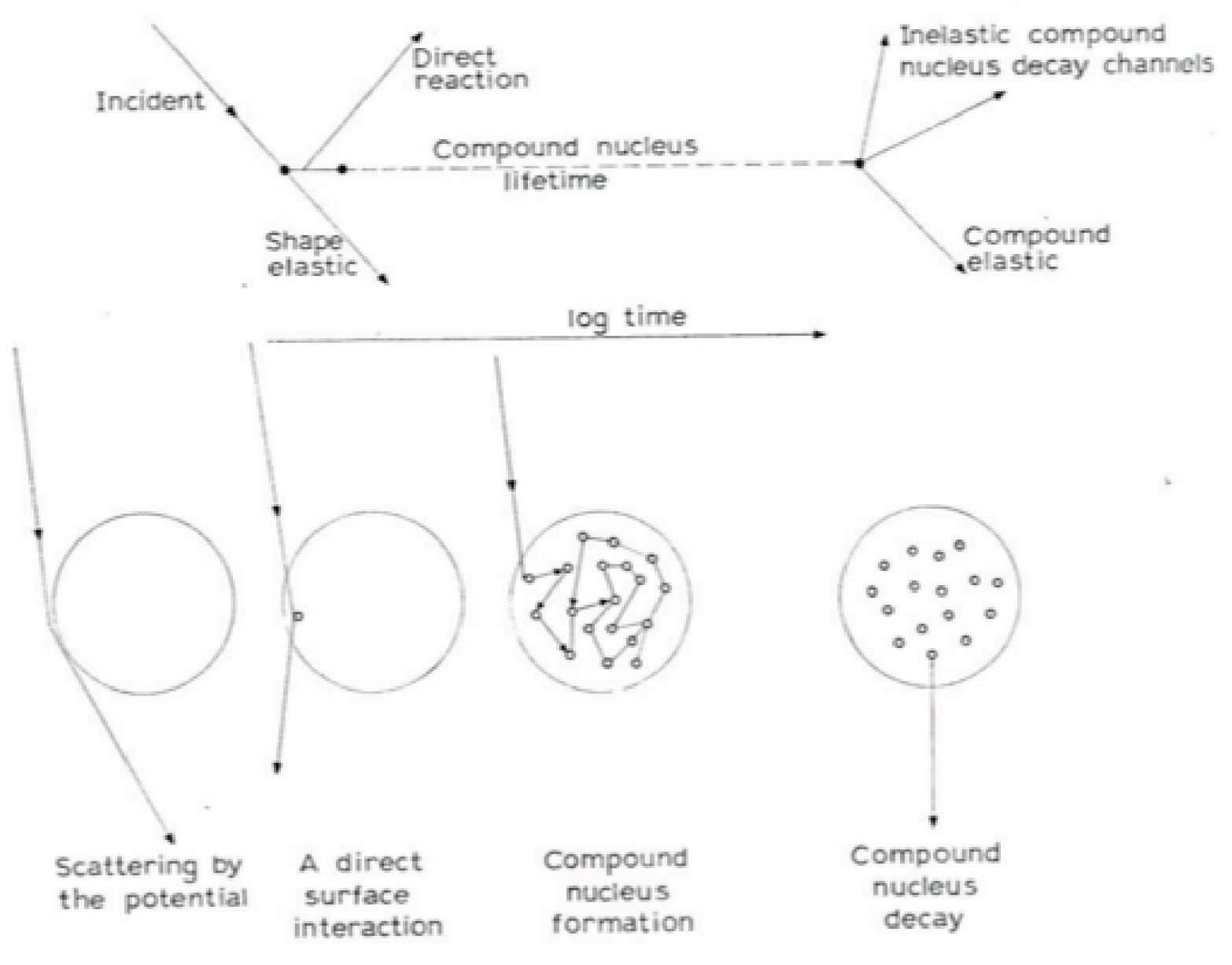




\section{Continuum or statistical theory of the compound nucleus}

In light nuclei, a reaction proceeds either directly to a bound state or through an isolated narrow resonance. With increasing excitation energies in the compound nucleus, or for medium- or heavymass nuclei, the resonances become broader and are located closer together. There is a continuous transition from sharp, isolated levels to the so-called continuum where levels overlap so much that little structure remains in the cross section. In other words, the cross section varies smoothly with energy. The reaction cross section needs to be averaged over all the existing levels which are described in terms of a level density (i.e the number of levels per energy interval) rather than individual levels.

The reaction cross section, averaged over many resonances, can be derived through the reaction model called the Hauser-Feshbach theory.

The final cross section is factorized in terms of a cross section for compound nucleus formation through the channel $\alpha$ and a branching ratio for decay into the channel $\alpha$ '

$$
\begin{gathered}
\sigma_{\left(\alpha, \alpha^{\prime}\right)}^{J \pi}=\sigma_{\alpha C}^{J \pi} \frac{G_{\alpha^{\prime}}^{J \pi}}{\sum_{\alpha^{\prime \prime}} G_{\alpha^{\prime \prime}}^{J \pi}} \\
\sigma_{\left(\alpha, \alpha^{\prime}\right)}=\frac{\pi}{k_{\alpha}^{2}} \sum_{J \pi} \frac{2 J+1}{\left(2 I_{1}+1\right)\left(2 I_{2}+1\right)} \frac{\sum_{s l} \hat{T}_{l}^{J}(\alpha) \times \sum_{s^{\prime} l^{\prime}} \hat{T}_{l^{\prime}}^{J}\left(\alpha^{\prime}\right)}{\sum_{\alpha^{\prime \prime}} \sum_{s^{\prime \prime} l^{\prime \prime}} \hat{T}_{l^{\prime \prime}}^{J}\left(\alpha^{\prime \prime}\right)}
\end{gathered}
$$


More schematically

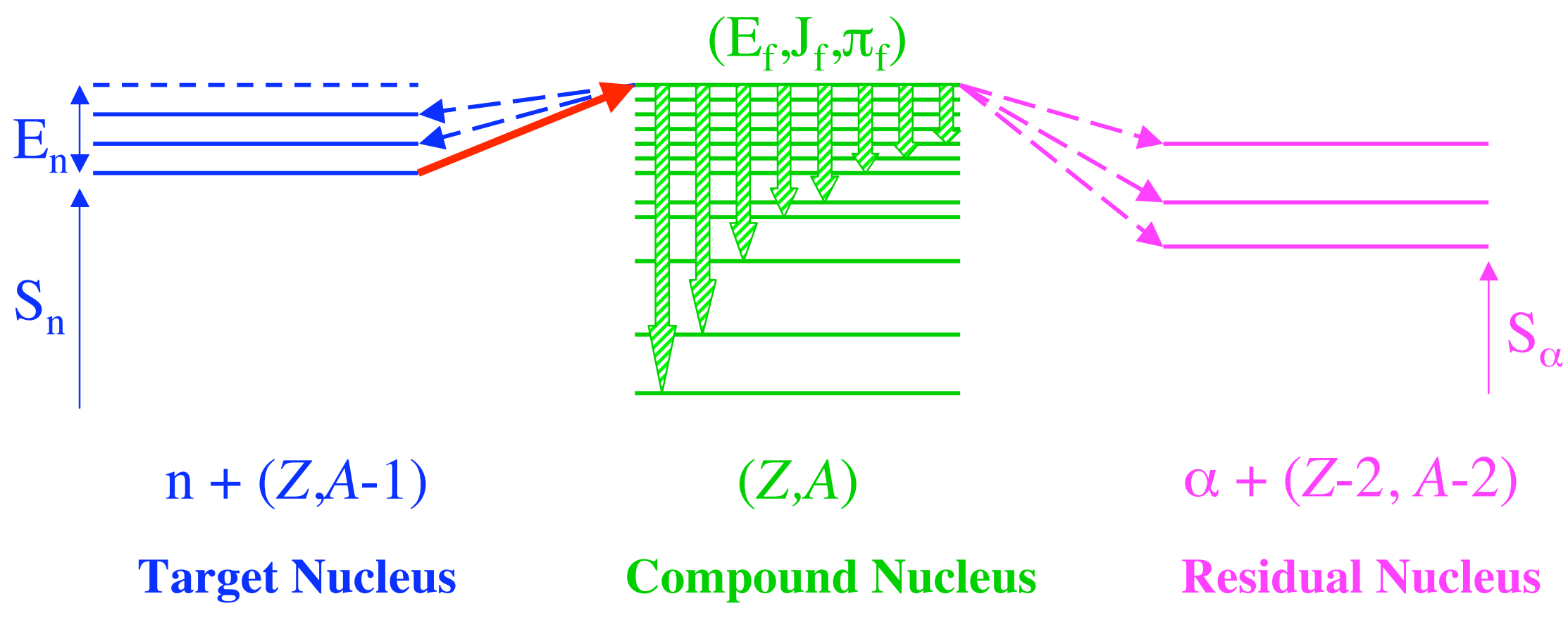

$$
\sigma_{(a, b)} \propto \sum_{J, \pi} \frac{T_{a}\left(J^{\pi}\right) T_{b}\left(J^{\pi}\right)}{T_{a}\left(J^{\pi}\right)+T_{b}\left(J^{\pi}\right)}
$$

$\boldsymbol{T}$ : Transmission coefficient, i.e the probability to favour a given channel $(\boldsymbol{a}, \boldsymbol{b}=\mathrm{n}, \mathrm{p}, \alpha, \gamma)$ 
The uncertainties involved in any HF cross section calculation are not related to the model of formation and de-excitation of the compound nucleus itself (except through the width fluctuation correction), but rather to the evaluation of the nuclear quantities necessary for the calculation of the transmission coefficients.

-The knowledge of the ground state properties (masses, deformations, matter densities) of the target and residual nuclei is indispensable.

- The excited state properties have also to be known. Experimental data may be scarce above some excitation energy, and especially so for nuclei located far from the valley of nuclear stability. This is why frequent resort to a level density prescription is mandatory.

- The transmission coefficients for particle emission are calculated by solving the Schrödinger equation with the appropriate optical potential for the particle-nucleus interaction.

-The photon transmission coefficient is calculated assuming the dominance of dipole E1 transitions (the M1 transitions are usually included as well, but do not contribute significantly). Reaction theory relates the $\gamma$-transmission coefficient for excited states to the ground state photoabsorption assuming the Giant Dipole Resonance to be built on each excited state. These resonances are classically estimated within a Lorentzian representation, at least for medium- and heavy-mass nuclei. Experimental photoabsorption data confirm the simple semi-classical prediction of a Lorentzian shape at energies around the resonance energy.

Note that the hypothesis of an equilibrium compound nucleus underlying the Hauser-Feschbach equation implies that its formation and decay are independent except for the basic requirements of conservation of energy and of the relevant quantum numbers. This may not be fully satisfied, particularly in cases where a few strongly and many weakly absorbing channels are mixed. As an example, the HauserFeschbach expression is known to fail when applied to the elastic channel for which the transmission coefficients for the entrance and exit channels are identical, hence correlated. To account for these deviations, so-called width fluctuation corrections can be introduced in the Hauser-Feshbach formalism by different approximate expressions. 


\section{Nuclear Ingredients required to calculate transmission coefficients}

1. Ground \& Excited state properties

- Ground state mass, equilibrium deformation, density distribution, shell energy, pairing energy, spl scheme, etc...

- Excited spectrum $(E, J, \pi)$ - Nuclear Level Densities $\rho(E, J, \pi)$

- Energy surfaces - Fission barrier \& width or fission path

2. Interaction properties

- Nucleon-nucleus optical potential

- Alpha-nucleus interaction potential

Solve Schrödinger equation with an

- $\gamma$-strength function: Giant Resonance Properties linked to photoabsorption

- Fission dynamics (neutron-induced, spontaneous fission)

$\longrightarrow$ Nuclear Ingredients from

(1) direct experimental data

(2) indirect (model-dep) exp. data

(3) theoretical models

For exotic nuclei, no data exists and calculation have to rely exclusively on theoretical models 


\section{Multiple Hauser-Feshbach}

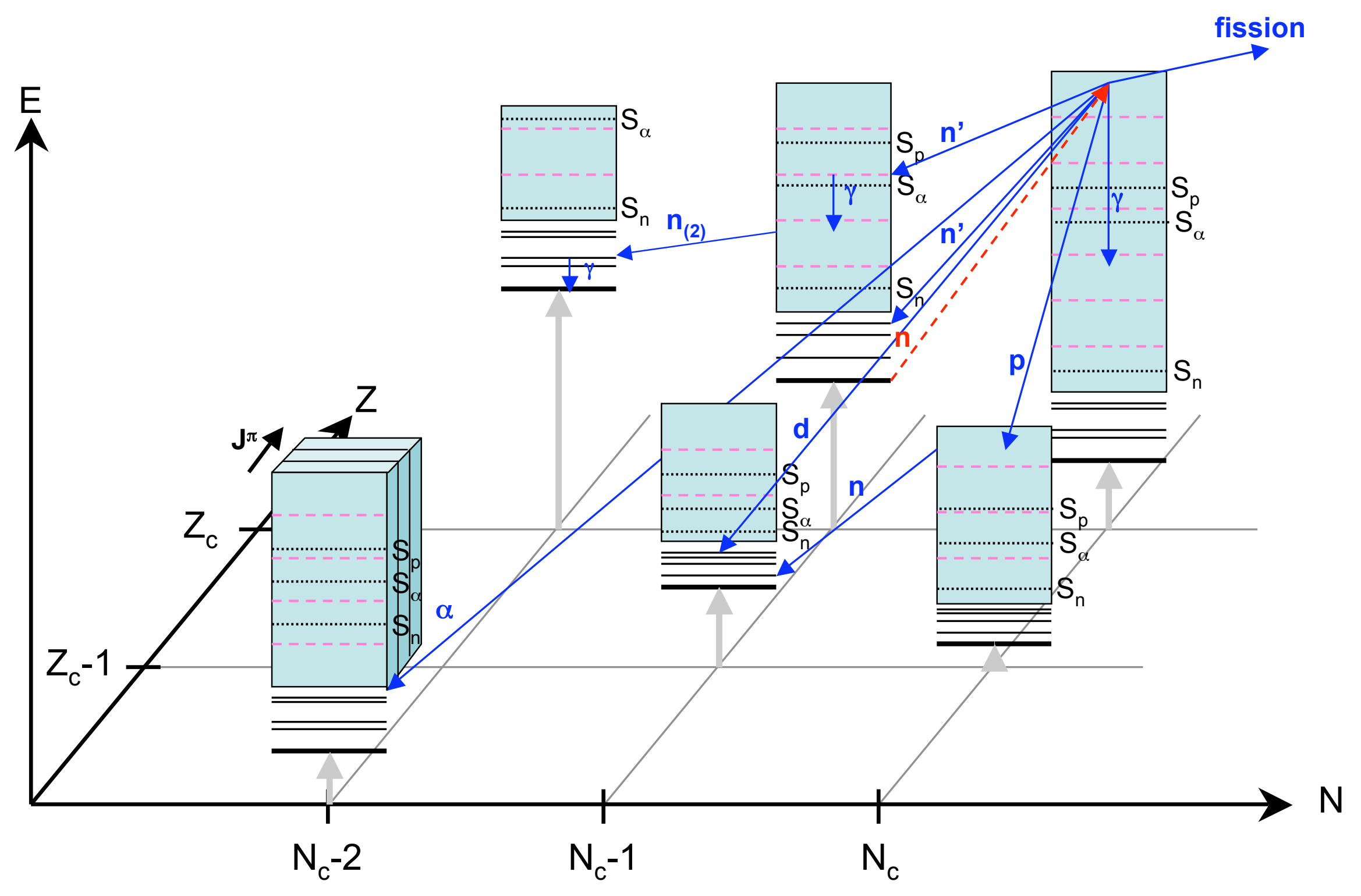




\section{Reaction rates in a thermalized plasma}

For a Maxwell-Boltzmann distribution of the relative energies at a temperature $T$, the rate is obtained by integrating the cross section over a Maxwell-Boltzmann distribution of energies $E$ at the given temperature. In addition, in hot astrophysical plasmas, a target nucleus exists in its ground as well as excited states, so that

$$
N_{\mathrm{A}}\langle\sigma v\rangle_{j l}^{*}(T)=\left(\frac{8}{\pi m}\right)^{1 / 2} \frac{N_{\mathrm{A}}}{(k T)^{3 / 2} G_{I}(T)} \int_{0}^{\infty} \sum_{\mu} \frac{\left(2 J_{I}^{\mu}+1\right)}{\left(2 J_{I}^{0}+1\right)} \sigma_{j l}^{\mu}(E) E \exp \left(-\frac{E+\varepsilon_{I}^{\mu}}{k T}\right) d E
$$

where $k$ is the Boltzmann constant, $m$ the reduced mass of the $I^{0}+j$ system, $N_{\mathrm{A}}$ the Avogadro number, and $G(T)$ the temperature-dependent normalised partition function given by

$$
G_{I}(T)=\sum_{\mu} \frac{2 J_{I}^{\mu}+1}{2 J_{I}^{0}+1} \exp \left(-\frac{\varepsilon_{I}^{\mu}}{k T}\right)
$$

Reverse reactions can be estimated with the use of the reciprocity theorem. In particular, the stellar photo-dissociation rates (in $\mathrm{s}^{-1}$ ) are classically derived from the reverse radiative capture rates by

$$
\lambda_{(\gamma, j)}^{*}(T)=\frac{\left(2 J_{I}^{0}+1\right)\left(2 J_{j}+1\right)}{\left(2 J_{L}^{0}+1\right)} \frac{G_{I}(T)}{G_{L}(T)}\left(\frac{m k T}{2 \pi \hbar^{2}}\right)^{3 / 2}\langle\sigma v\rangle_{(j, \gamma)}^{*} \mathrm{e}^{-Q_{j \gamma} / k T}
$$

where $Q_{j \gamma}$ is the $Q$-value of the $I^{0}(j, \gamma) L^{0}$ capture reaction.

Note that, in stellar conditions, the reaction rates for targets in thermal equilibrium obey reciprocity since the forward and reverse channels are symmetrical, in contrast to the situation which would be encountered for targets in their ground states only.

Few reaction codes specifically adapted to estimate reaction rates: Smoker, Most, Non-Smoker, ...TALYS 


\section{TALYS reaction code}

(Koning \& Hilaire)

Added-values of the TALYS code in comparison with SMOKER, MOST, NON-SMOKER:

- design for nuclear applications (large energy range: $1 \mathrm{keV}-200 \mathrm{MeV}$ )

- inclusion of pre-equilibrium reaction mechanism

- the detailed description of the decay scheme, including the description of $\gamma$-delayed particle emission

- inclusion of multi-particle emission (--> calculation of the Maxwellianaveraged $(\mathrm{n}, 2 \mathrm{n})$ rate of relevance at $\left.T_{9}>2\right)$

- inclusion of detailed width fluctuation corrections

- inclusion of parity-dependent level densities (in the full jls HF scheme)

- state-of-the-art scattering calculations (ECIS 2006)

- inclusion of coupled channels description for deformed nuclei

- the inclusion of the fission channel for the compound as well as the residual nuclei.

Recent developments made

- to include the calculation of astrophysical rates

- to include the calculation of rates with "microscopic" ingredients

- to test approximations made by former codes

... and open source ... freely available at the website: http://www.talys.eu 
Comparaison of the known $(n, \gamma)$ reaction rates with the statistical model estimate

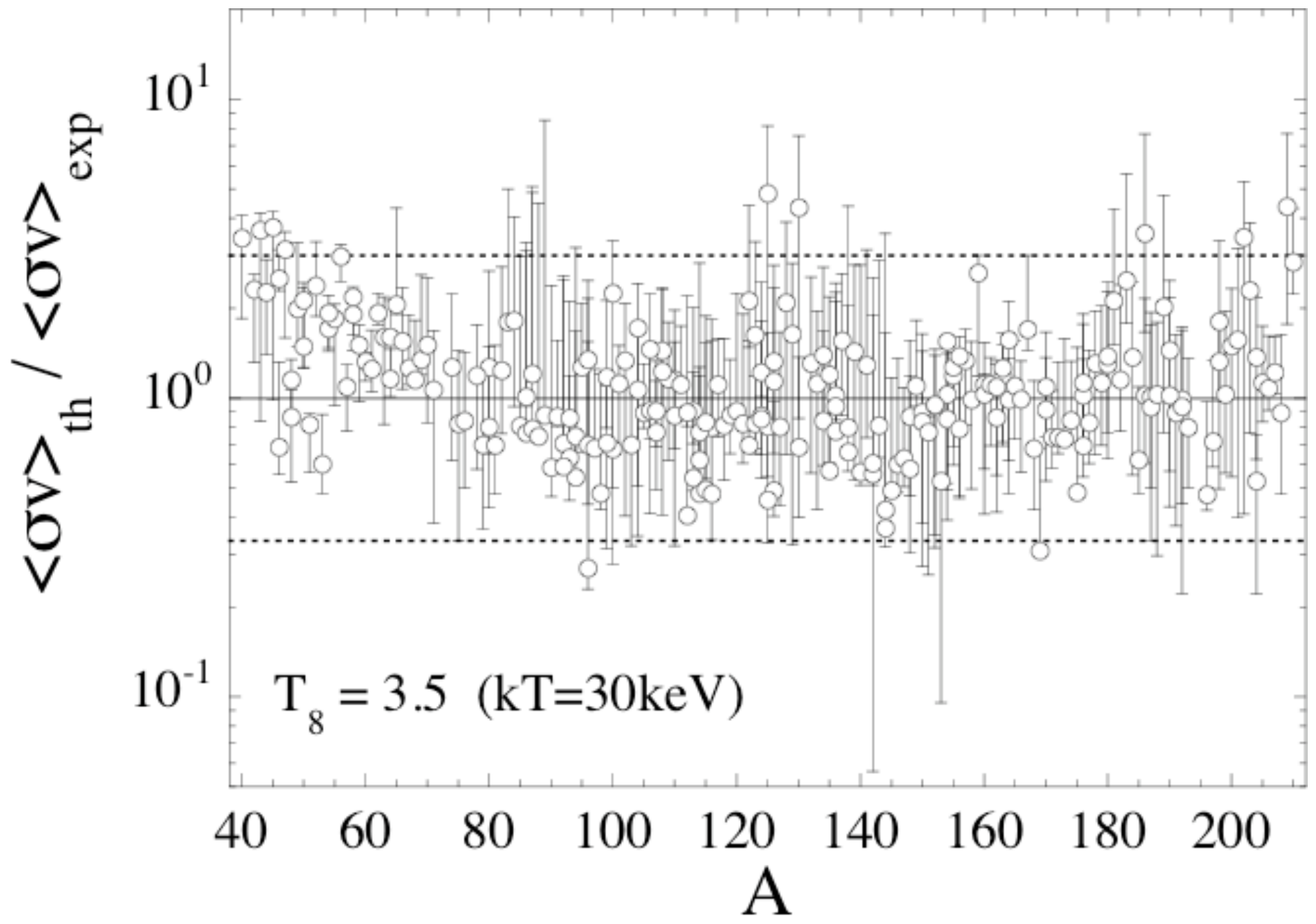


Comparaison of some $(p, \gamma)$ reaction cross section estimated within the statistical model
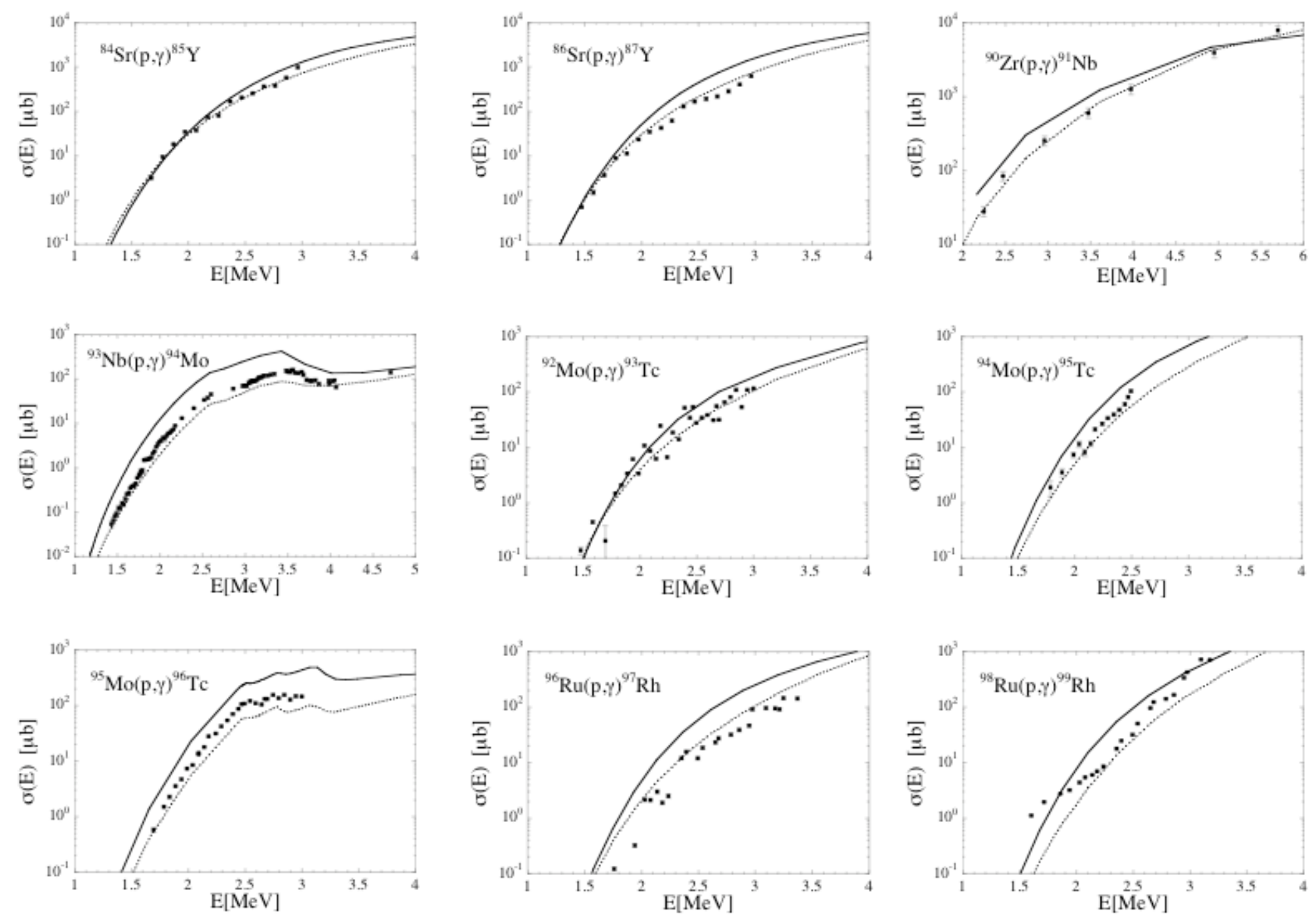

with 2 different proton-nucleus optical potentials: JLMB (solid line) and JLM (dotted line) 
Comparaison of some $(\alpha, \gamma)$ reaction cross section estimated within the statistical model
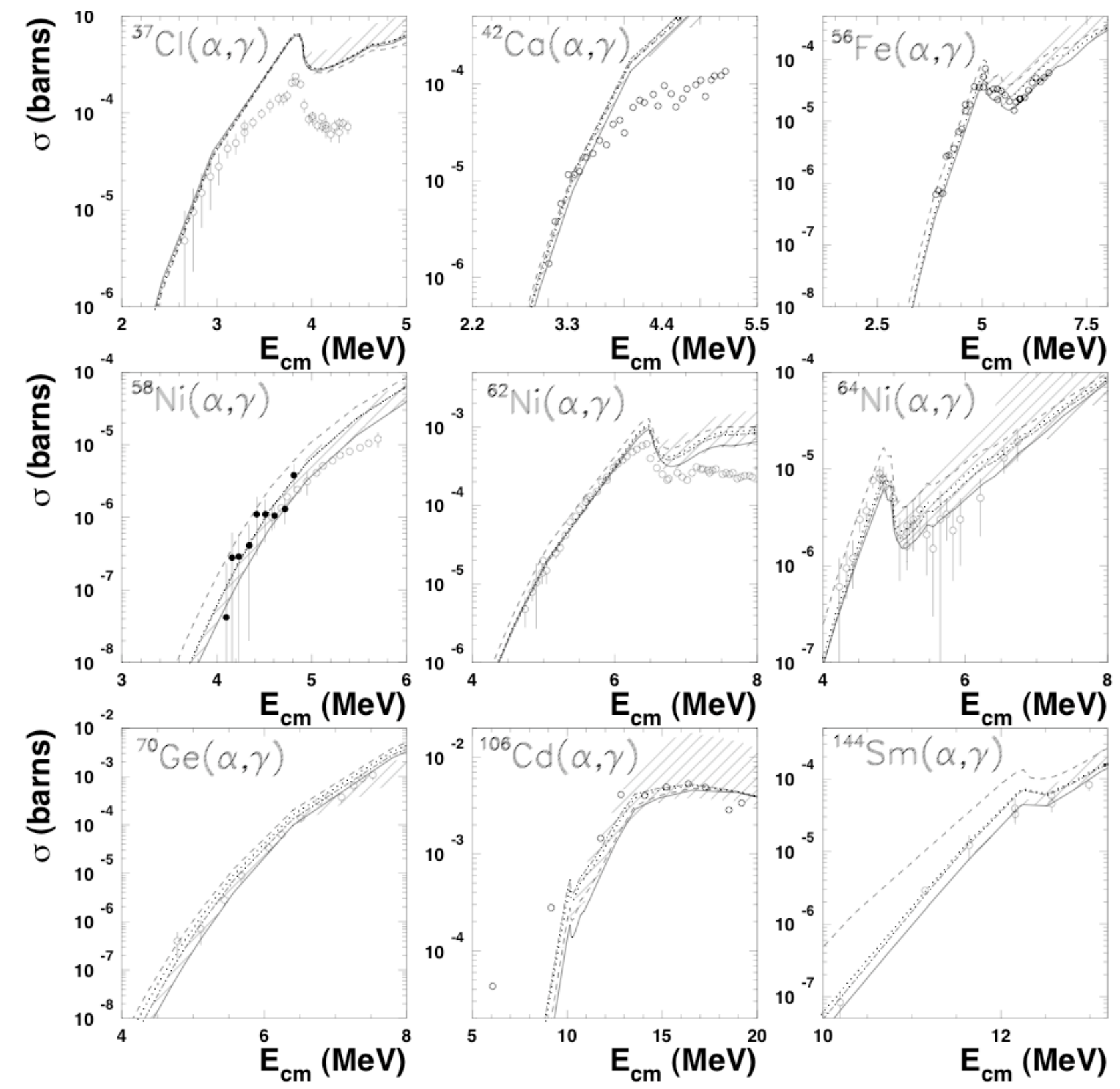


\section{The pre-equilibrium contribution to the reaction mechanism}




\section{Models sequence}

Shape elastic

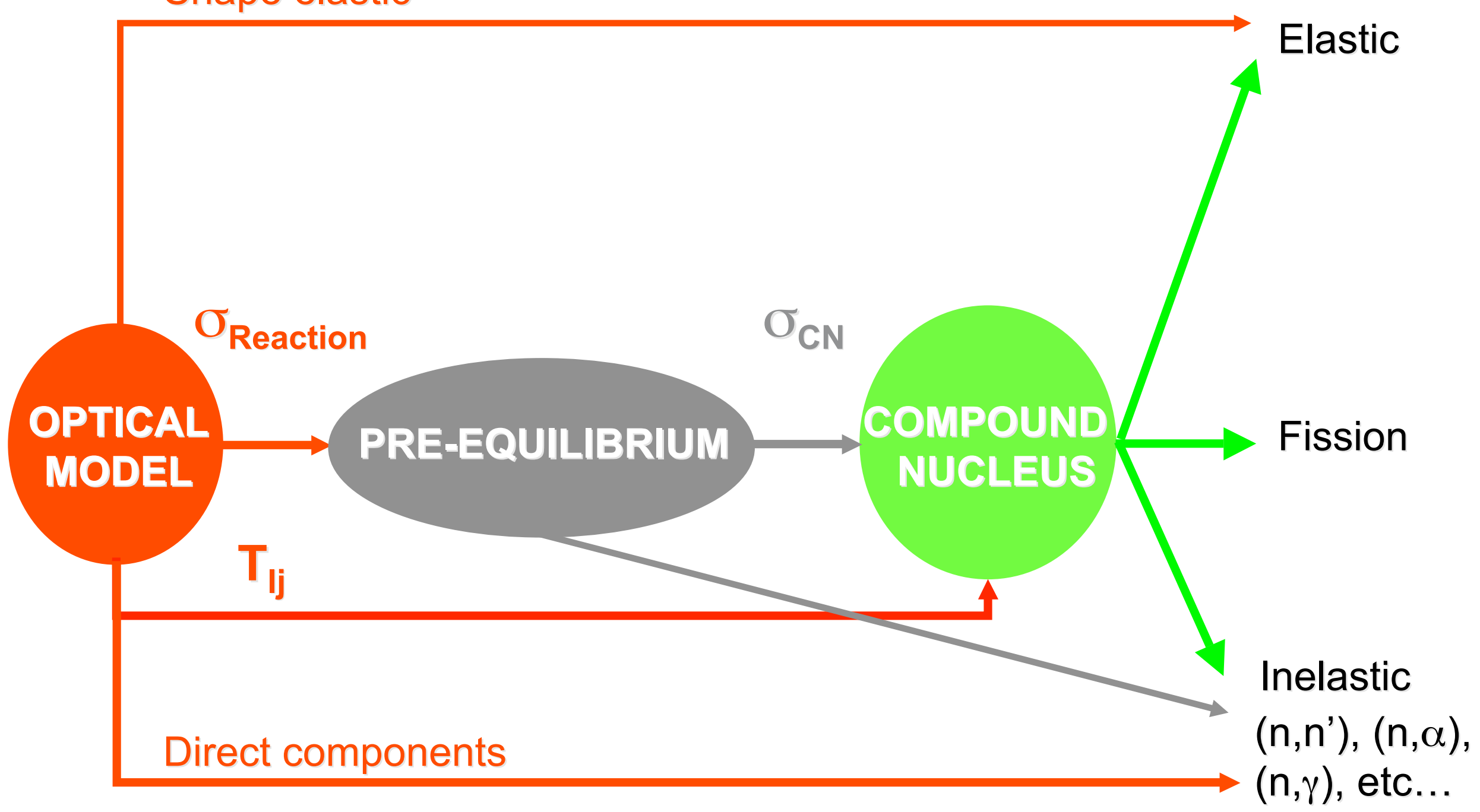




\section{Pre-equilibrium model(s)}

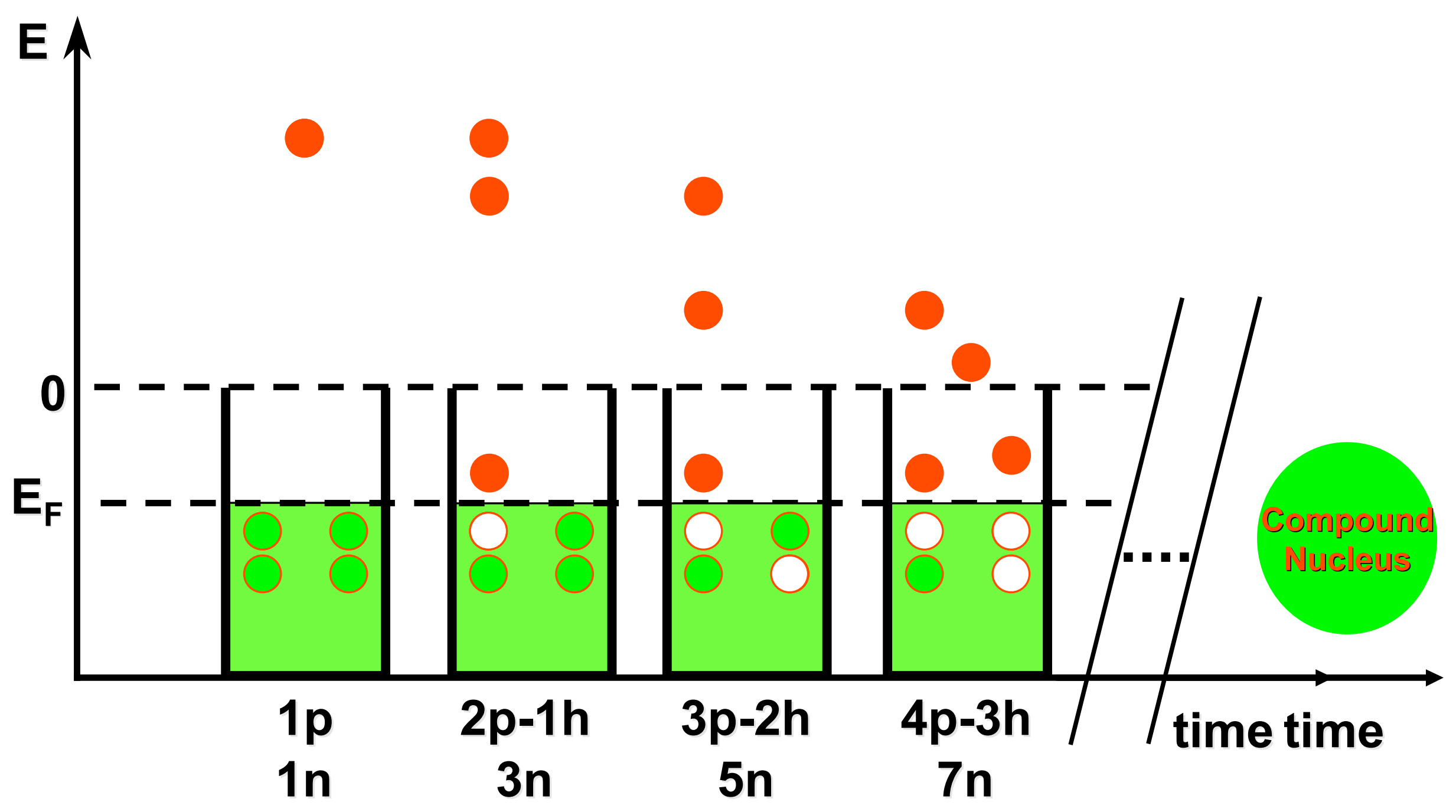




\section{Pre-equilibrium exciton model}

$P(n, E, t)=$ Probability to find at a given time $t$ the composite system with an energy $E$ and an excitons number $n$.

$\lambda_{a, b}(E)=$ Transition rate from an initial state a towards a state $b$ for a given energy $E$.

\section{Evolution equation}

$$
\begin{array}{r}
\frac{d P(n, E, t)}{d t}=P(n-2, E, t) \lambda_{n-2, n}(E)+P(n+2, E, t) \lambda_{n+2, n}(E) \\
-P(n, E, t)\left[\lambda_{n, n+2}(E)+\lambda_{n, n-2}(E)+\lambda_{n, \text { emiss }}(E)\right] \\
\text { Emission cross section in channel } C \\
\sigma_{c}\left(E, \varepsilon_{c}\right) d \varepsilon_{c}=\sigma_{R} \int_{0}^{t_{e q}} \sum_{n, \Delta n=2} P(n, E, t) \lambda_{n, c}(E) d t d \varepsilon_{c}
\end{array}
$$




\section{The pre-equilibrium contribution to the reaction mechanism}
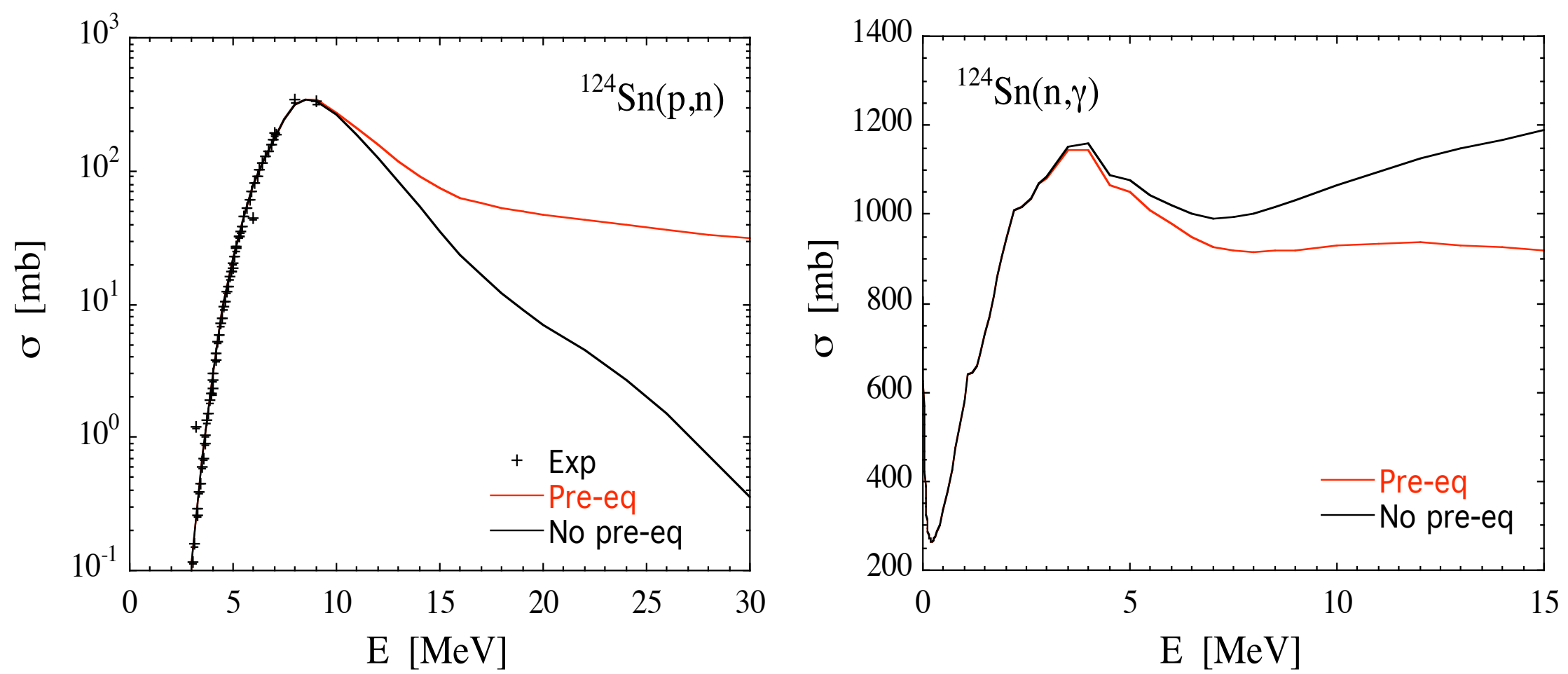


\section{Pre-equilibrium model}

$14 \mathrm{MeV}$ neutron $+{ }^{93} \mathrm{Nb}$

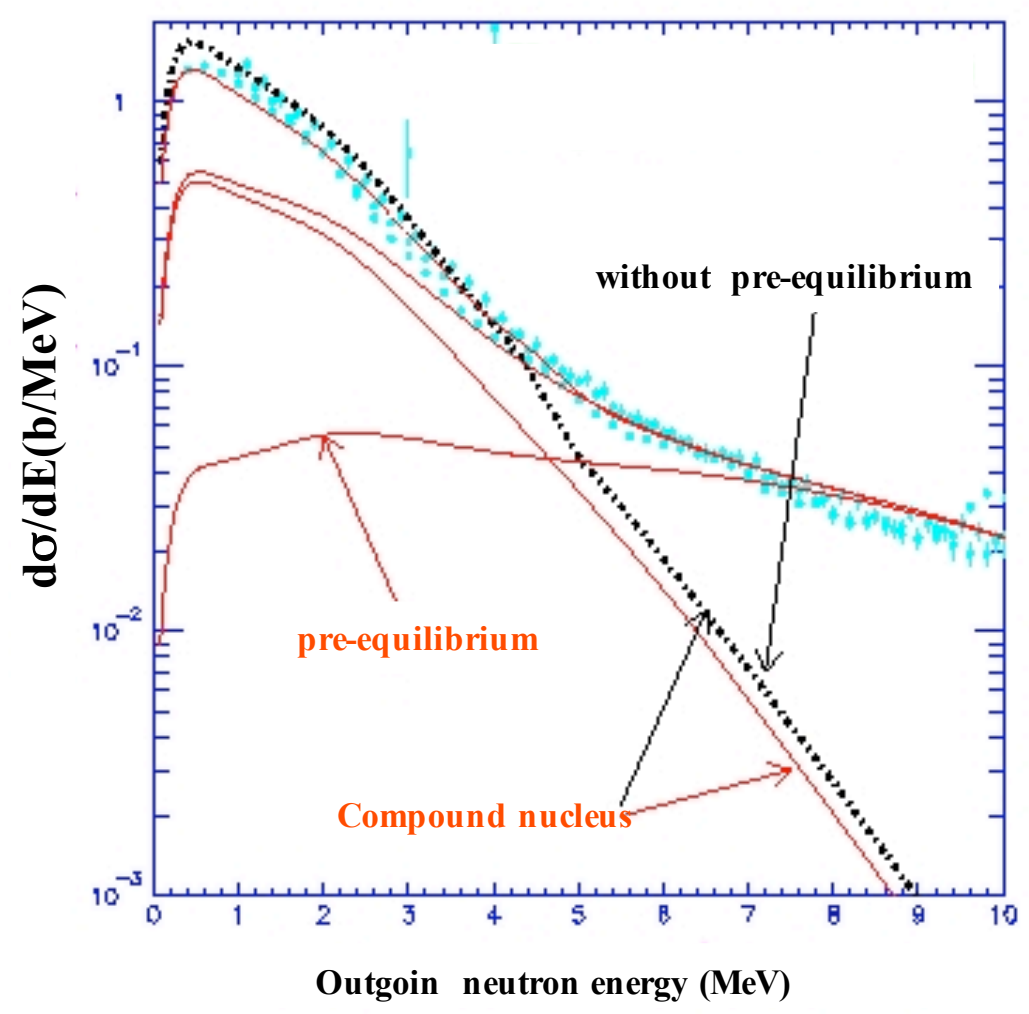

"n without pre-equilibrium

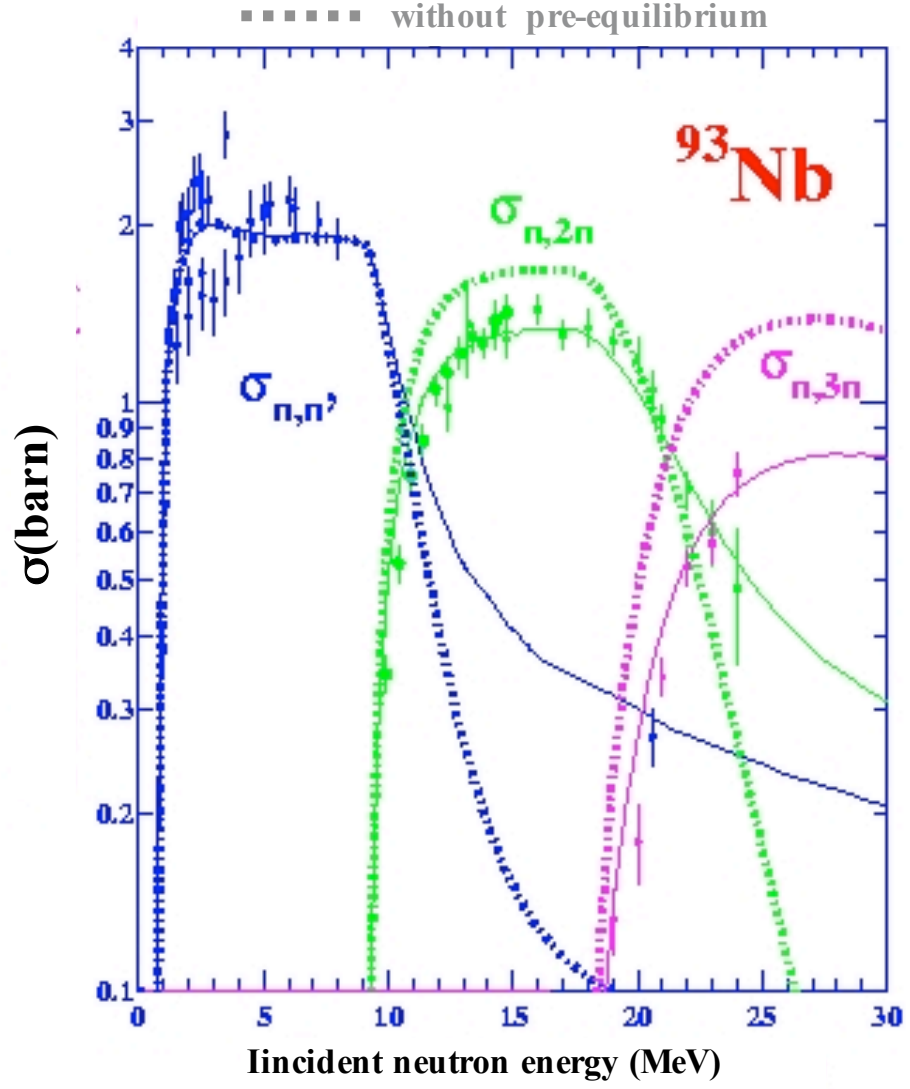




\section{The pre-equilibrium contribution to the reaction mechanism}

Pre-equilibrium emission takes place after the first stage of the reaction, long before statististical equilibrium is achieved. The incident neutron creates step-by-step more complex states and gradually loses the memory of its initial energy and direction. The pre-equilibrium contribution is responsible for the experimentally observed highenergy $(\sim 10 \mathrm{MeV})$ tail $[(\mathrm{p}, \mathrm{n}) ;(\mathrm{p}, \mathrm{pn})]$ and forward-peaked angular distribution $[(\mathrm{n}, \mathrm{xn})]$.
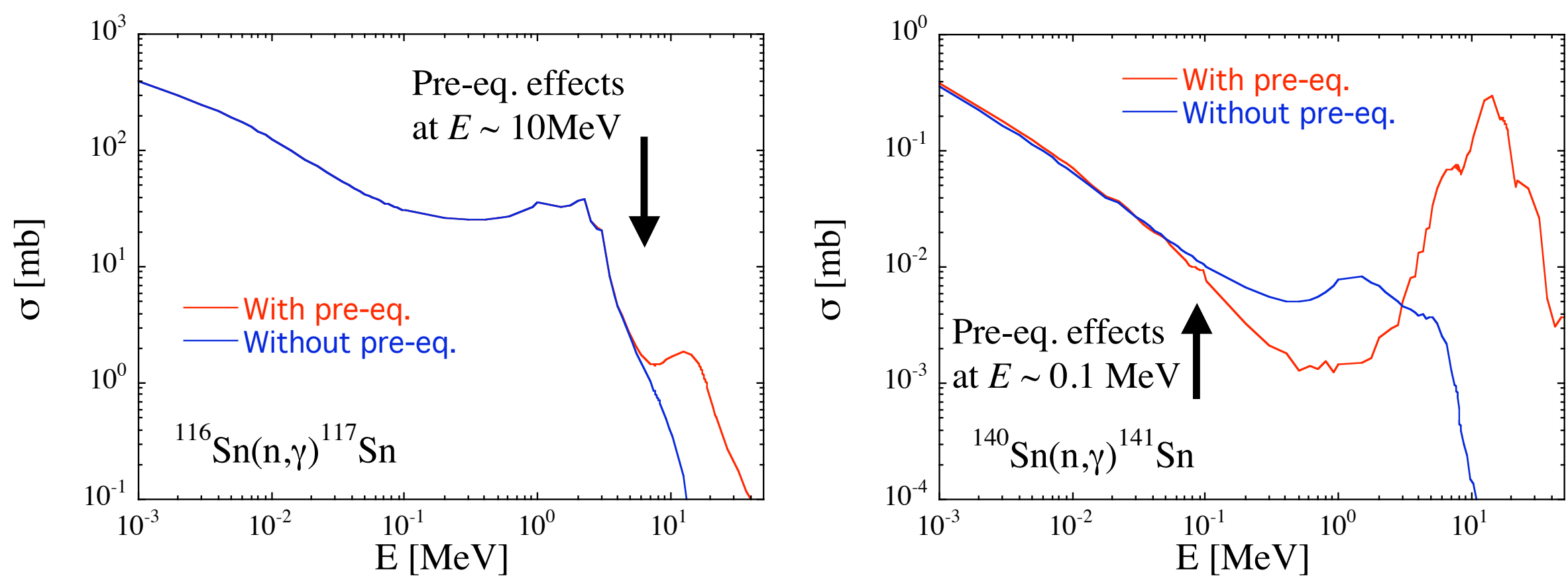

For stable nuclei, essentially contributes at energies around $\sim 10 \mathrm{MeV} \ldots$

... But for exotic nuclei, pre-equilibrium component can dominate the reaction mechanism already at low energies $(E \geq 100 \mathrm{keV})$ (thermodynamic equilibration cannot be reached). 
Impact of the pre-equilibrium contribution on the $(n, \gamma)$ rates

Neutron capture astrophysical rates for $\mathrm{Sn}$

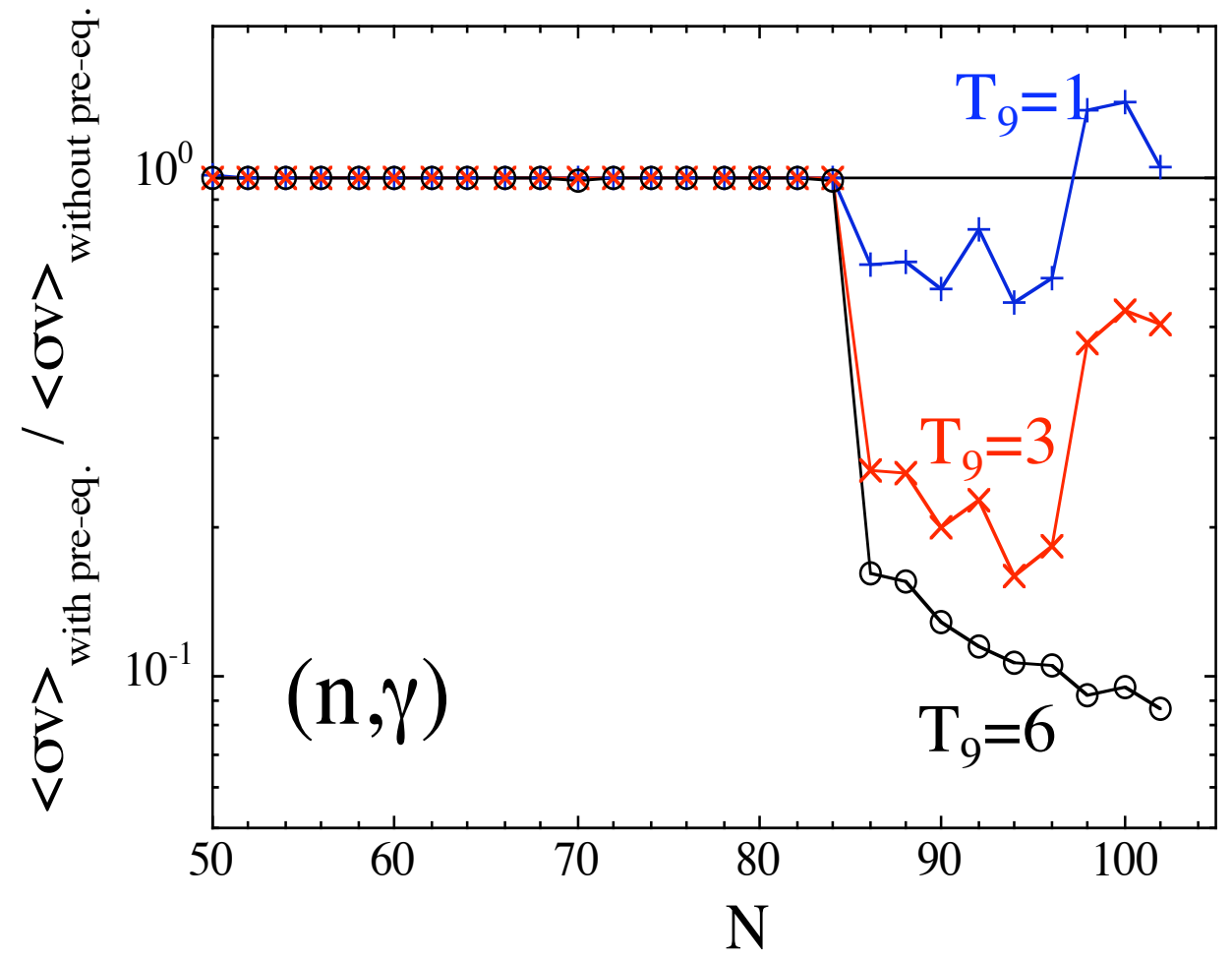

But still a lot of phenomenology in the pre-equilibrium model

$-->$ requires - particle-hole state density $\omega\left(\mathrm{p}_{\pi}, \mathrm{h}_{\pi}, \mathrm{p}_{v}, \mathrm{~h}_{v}, \mathrm{E}_{\mathrm{x}}\right)$

- transition rates expressed in terms of an effective square matrix element (effective residual interaction) or of the depth of the imaginary optical potential

Still requires further theoretical developments for exotic nuclei 


\section{Direct captures}

Direct scatter of incoming neutrons into a bound state without formation of a Compound Nucleus (particularly important for light and low- $\mathrm{S}_{\mathrm{n}} \mathrm{n}$-rich nuclei)

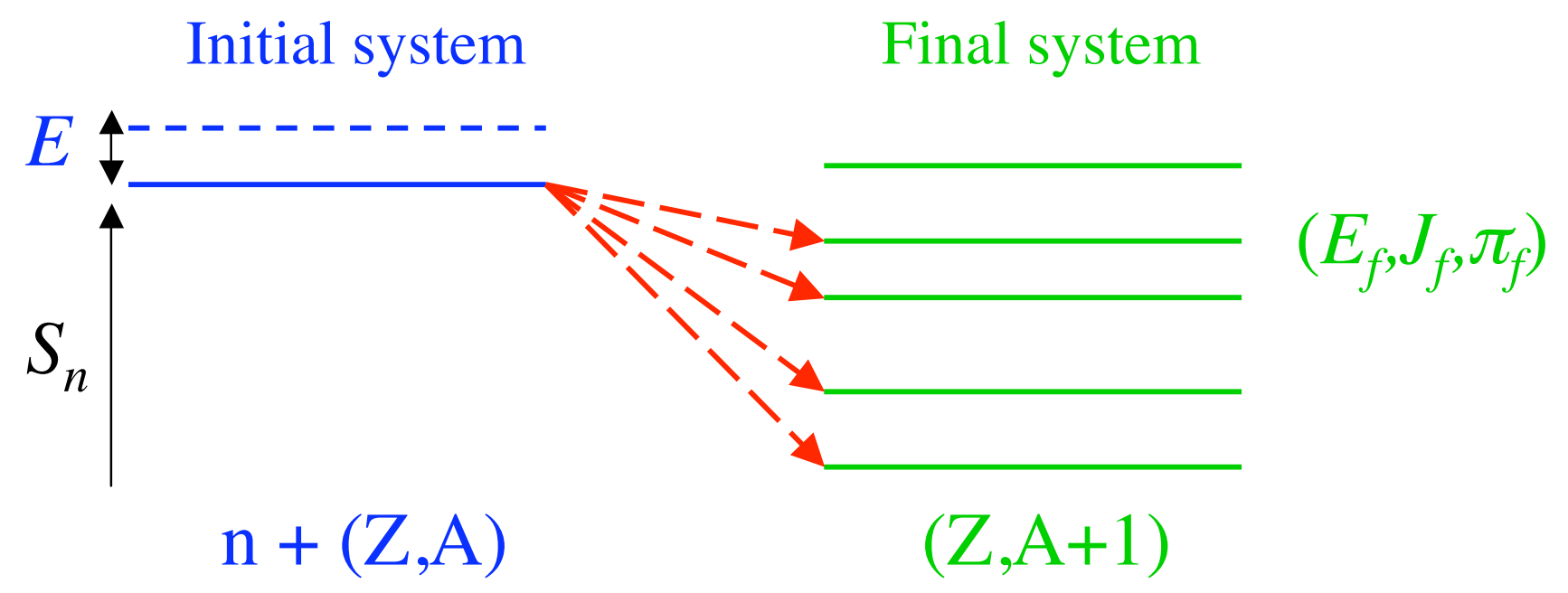

Direct capture cross section calculated within the potential model $\sigma_{f}^{D C}(E)=\frac{16 \pi}{9 \hbar} k_{\gamma}^{3} \bar{e}^{2}\left|Q_{i \rightarrow f}^{E 1}(E)\right|^{2} \quad$ with $\quad Q_{i \rightarrow f}^{E 1}(E)=\left\langle\Psi_{f}\left|T^{E 1}\right| \Psi_{i}(E)\right\rangle$ $\longrightarrow$ reliable model, but requires a proper description of

- n-nucleus potential

- excitation spectrum $\left(E_{f}, J_{f}, \pi_{f}\right)$

- spectroscopic factor $C^{2} S$

Overlap between the antisymmetrized wave function of the initial system $(Z, N)+n$ and the final state $f$ in $(Z, N+1)$ 


\section{Uncertainties from the determination of the spectrum $\left(E_{f}, J_{f}, \pi_{f}\right)$}

Unknown levels estimated with a Combinatorial NLD:

- 1 neutron p-h excitations $\left(C^{2} S=1\right)$

- full intrinsic (all n-p ph excitations) NLD with an average $\left\langle C^{2} S\right\rangle=0.5$

$$
\sigma^{D C}(E)=\sum_{f=0}^{x} C_{f}^{2} S_{f} \sigma_{f}^{D C}(E)+\sum_{E_{f}, J_{f}, \pi_{f}}\left\langle C^{2} S\right\rangle \mathcal{N}\left(E_{f}, J_{f}, \pi_{f}\right) \sigma_{f}^{D C}(E)
$$

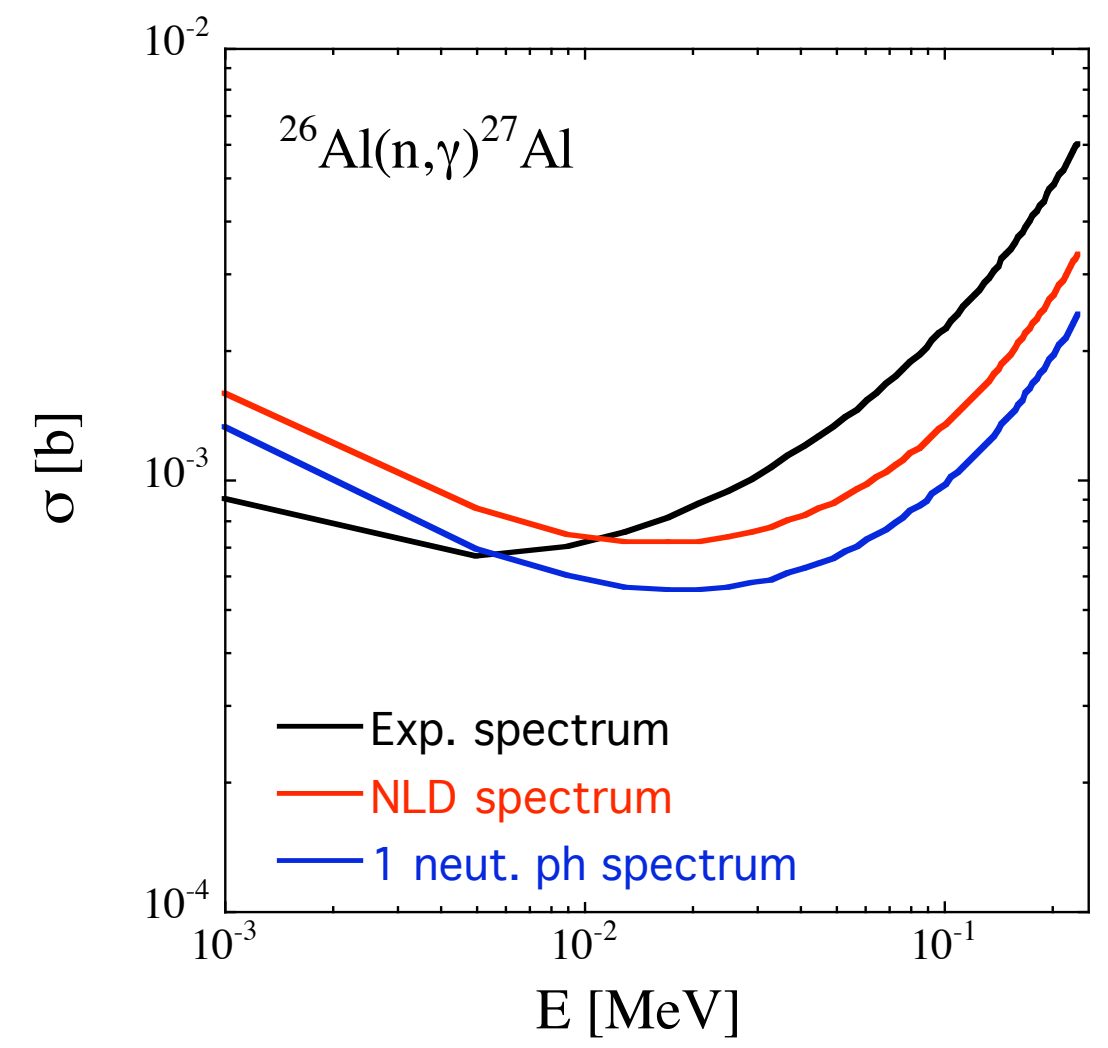

${ }^{27} \mathrm{Al}: 126$ exp. levels up to $S_{n}=13 \mathrm{MeV}$

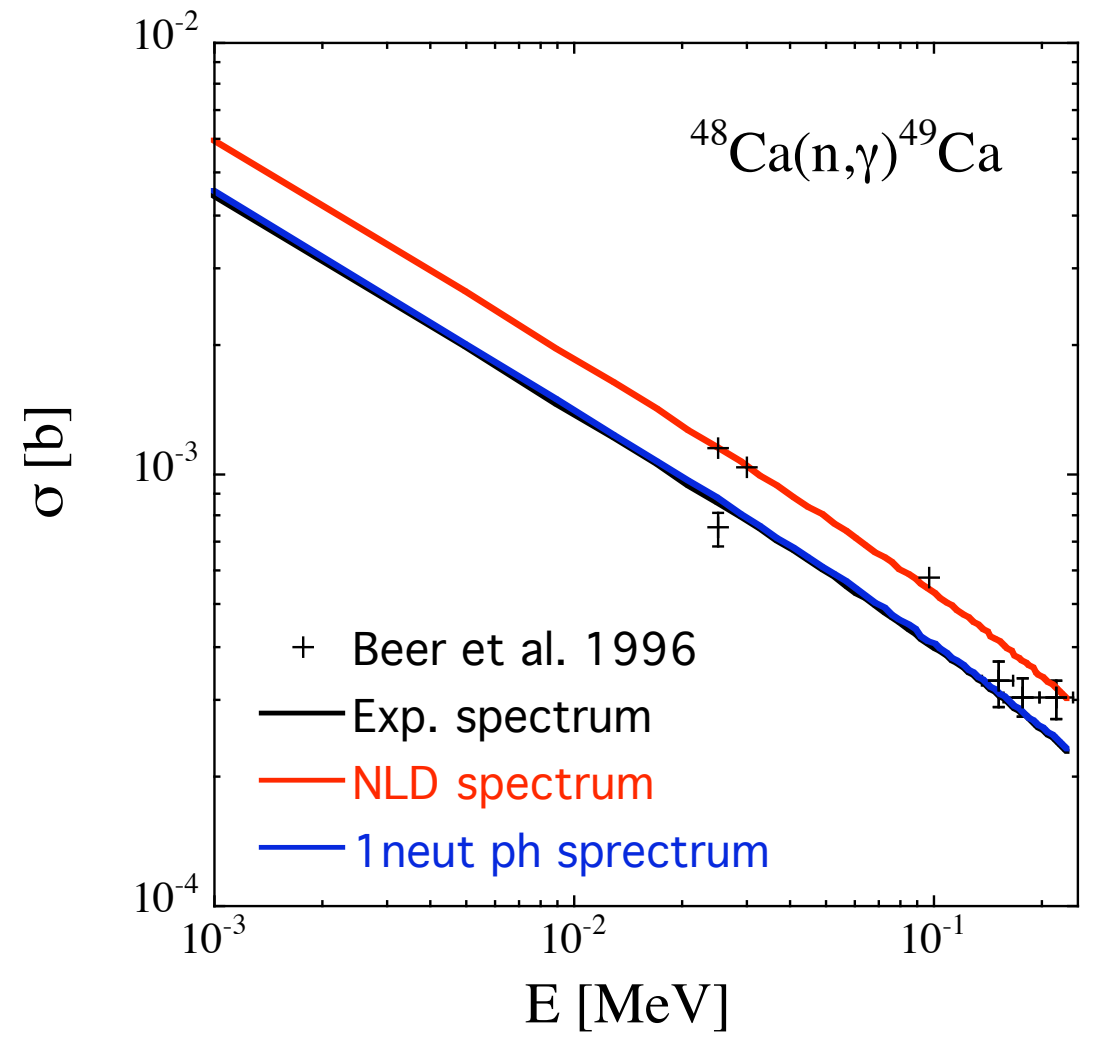

${ }^{49} \mathrm{Ca}: 13$ exp. levels up to $\mathrm{S}_{\mathrm{n}}=5.1 \mathrm{MeV}$ 


\section{Uncertainties from the determination of the spectrum $\left(E_{f}, J_{f}, \pi_{f}\right)$}

Unknown levels estimated with a Combinatorial NLD:

- 1 neutron p-h excitations $\left(C^{2} S=1\right)$

- full intrinsic (all n-p ph excitations) NLD with an average $\left\langle C^{2} S\right\rangle=0.5$

$$
\sigma^{D C}(E)=\sum_{f=0}^{x} C_{f}^{2} S_{f} \sigma_{f}^{D C}(E)+\sum_{E_{f}, J_{f}, \pi_{f}}\left\langle C^{2} S\right\rangle \mathcal{N}\left(E_{f}, J_{f}, \pi_{f}\right) \sigma_{f}^{D C}(E)
$$

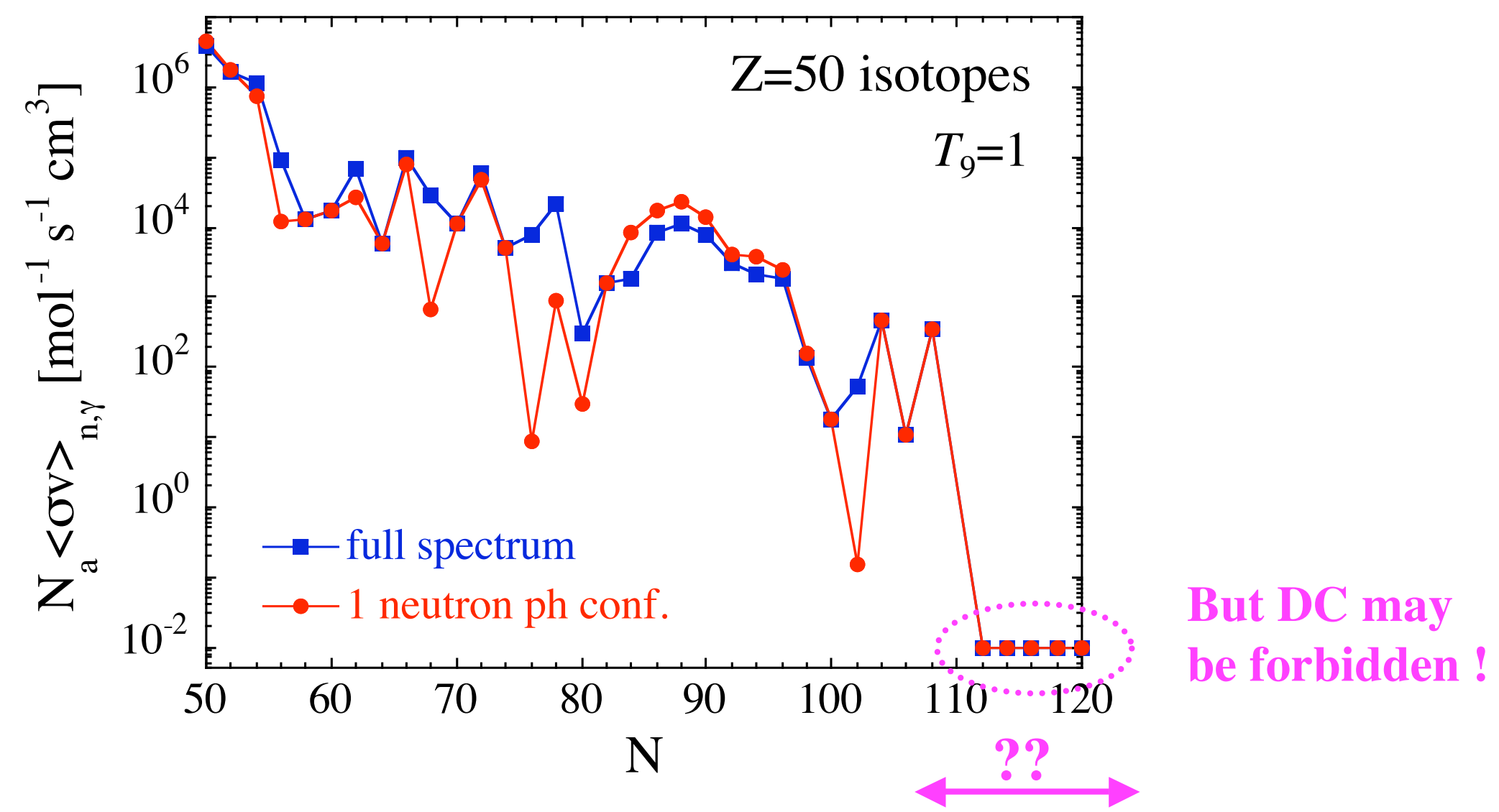


Impact of the n-nucleus potential on the DC rates

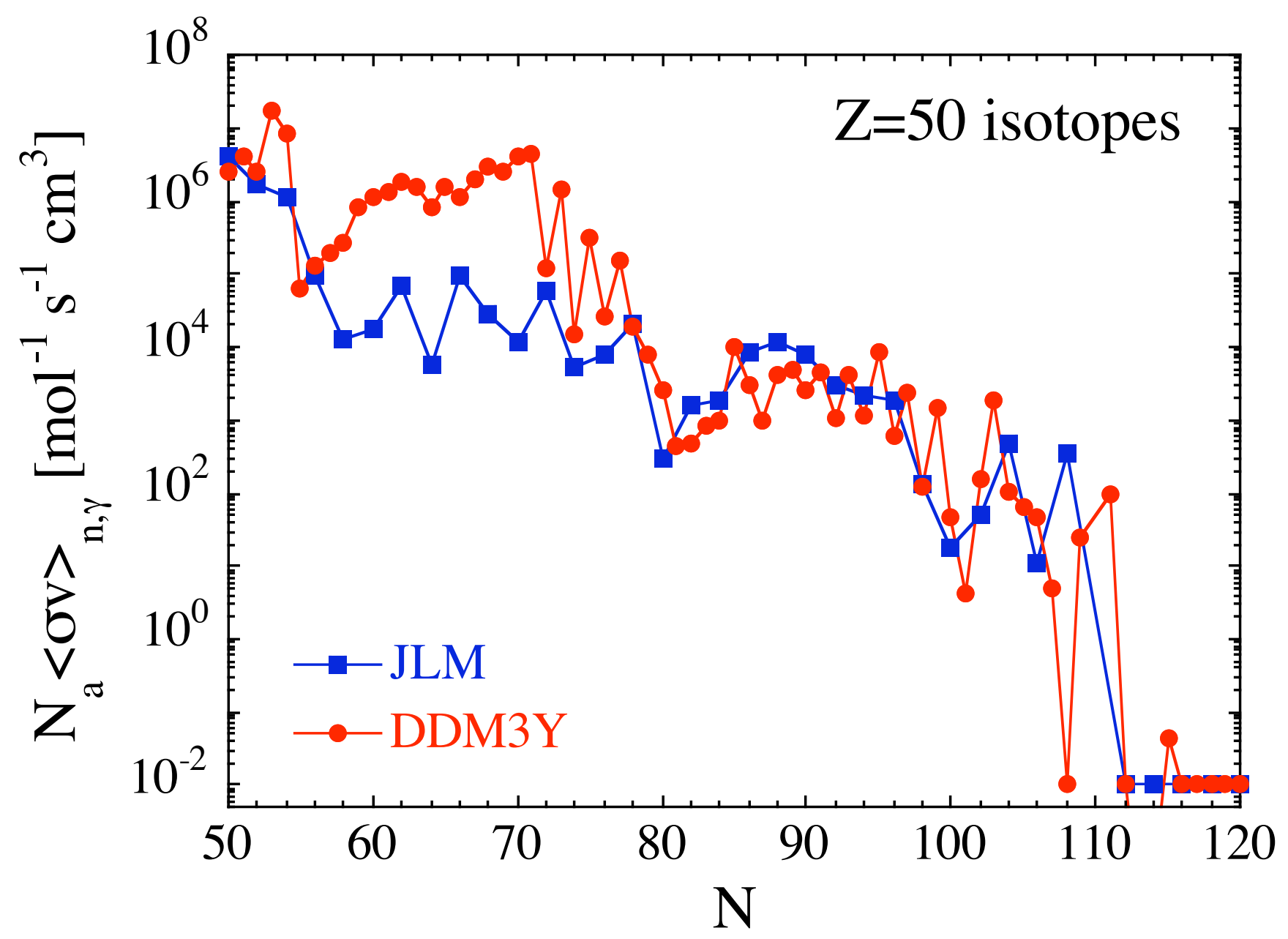


Spectroscopic factors extracted from $(\mathrm{d}, \mathrm{p})$ reactions (146 nuclei; 4270 nuclear levels)

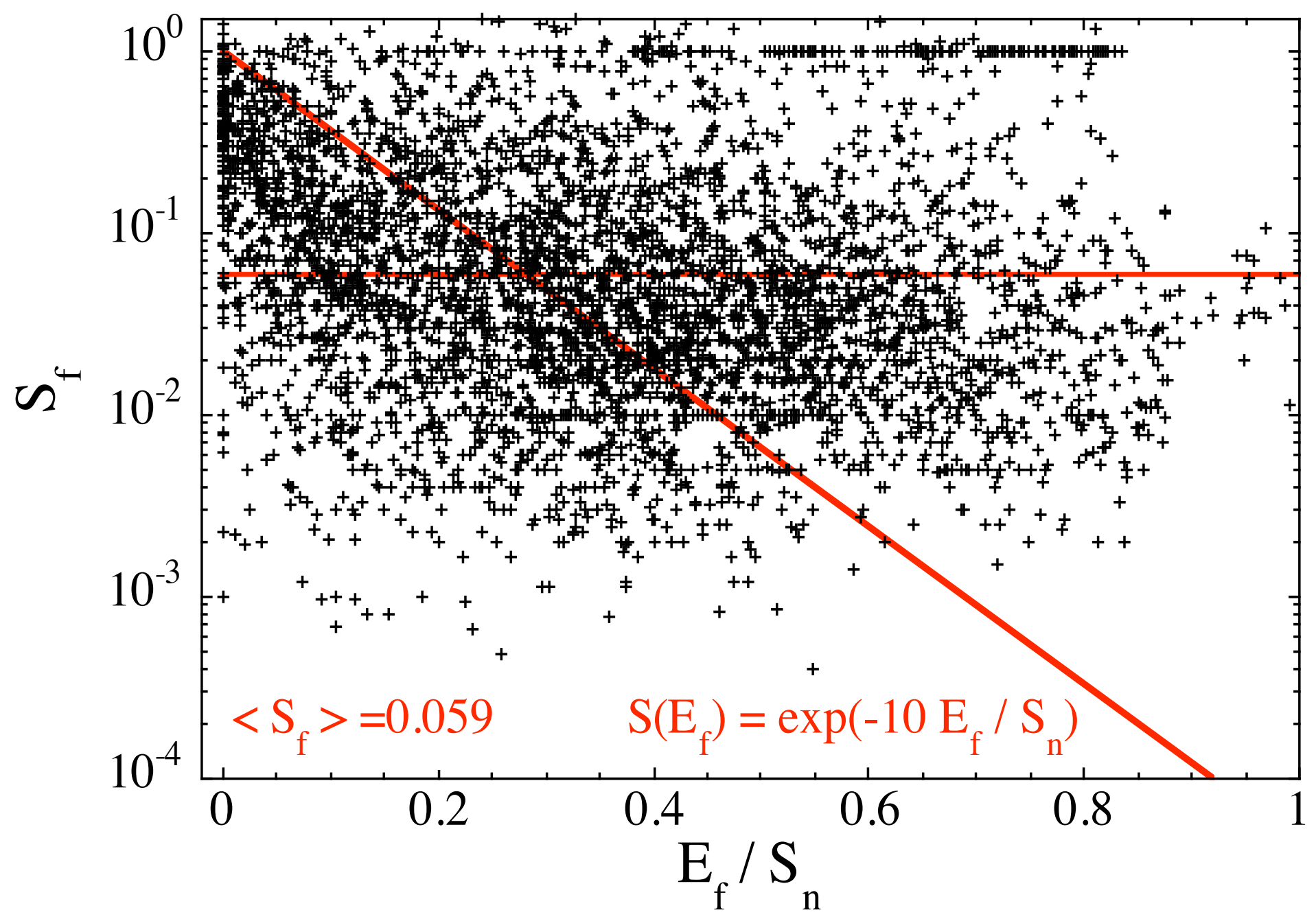

No global calculations so far to estimate systematically spectroscopic factors 
Final $(\mathbf{n}, \gamma)$ rates remain rather uncertain

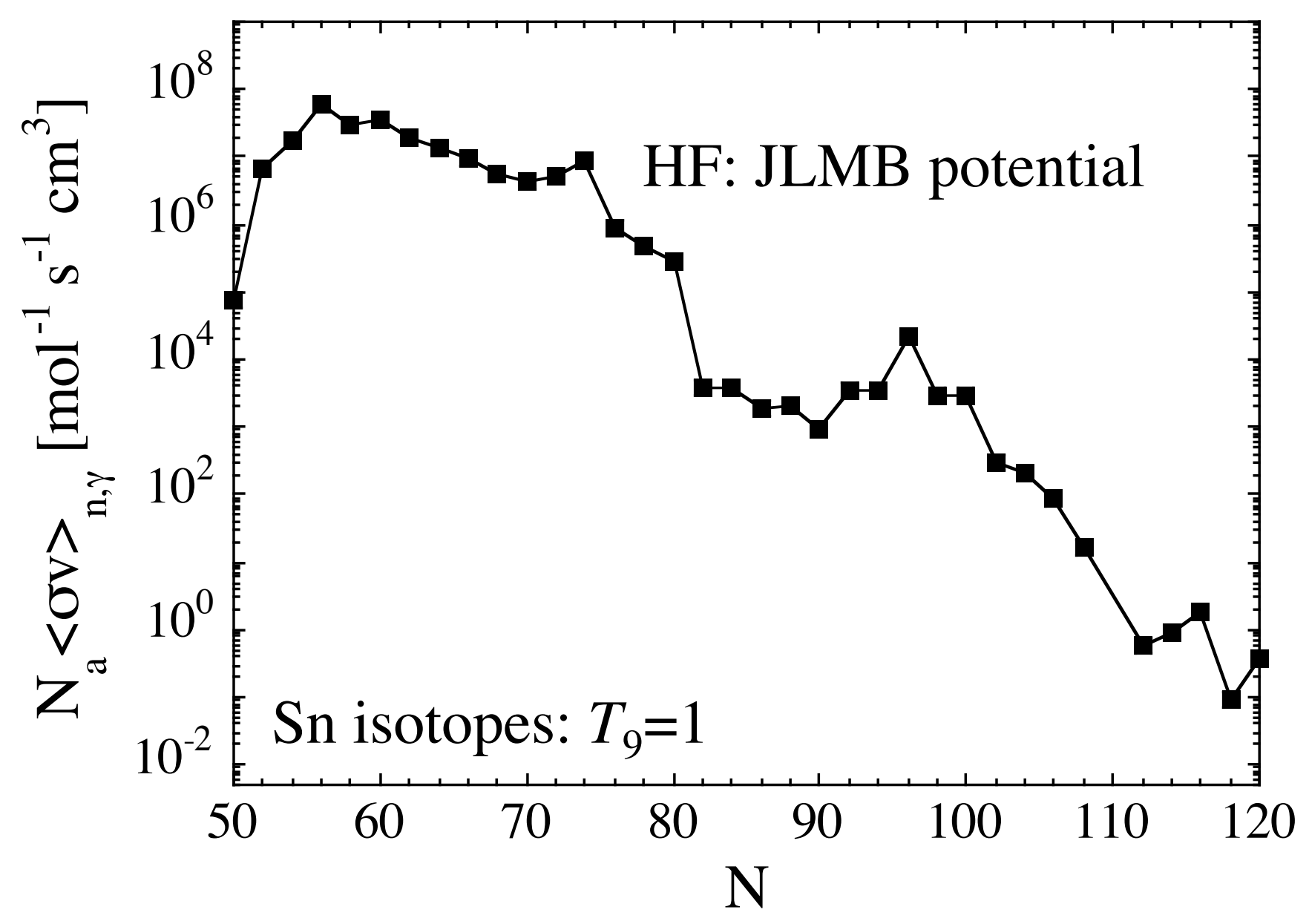




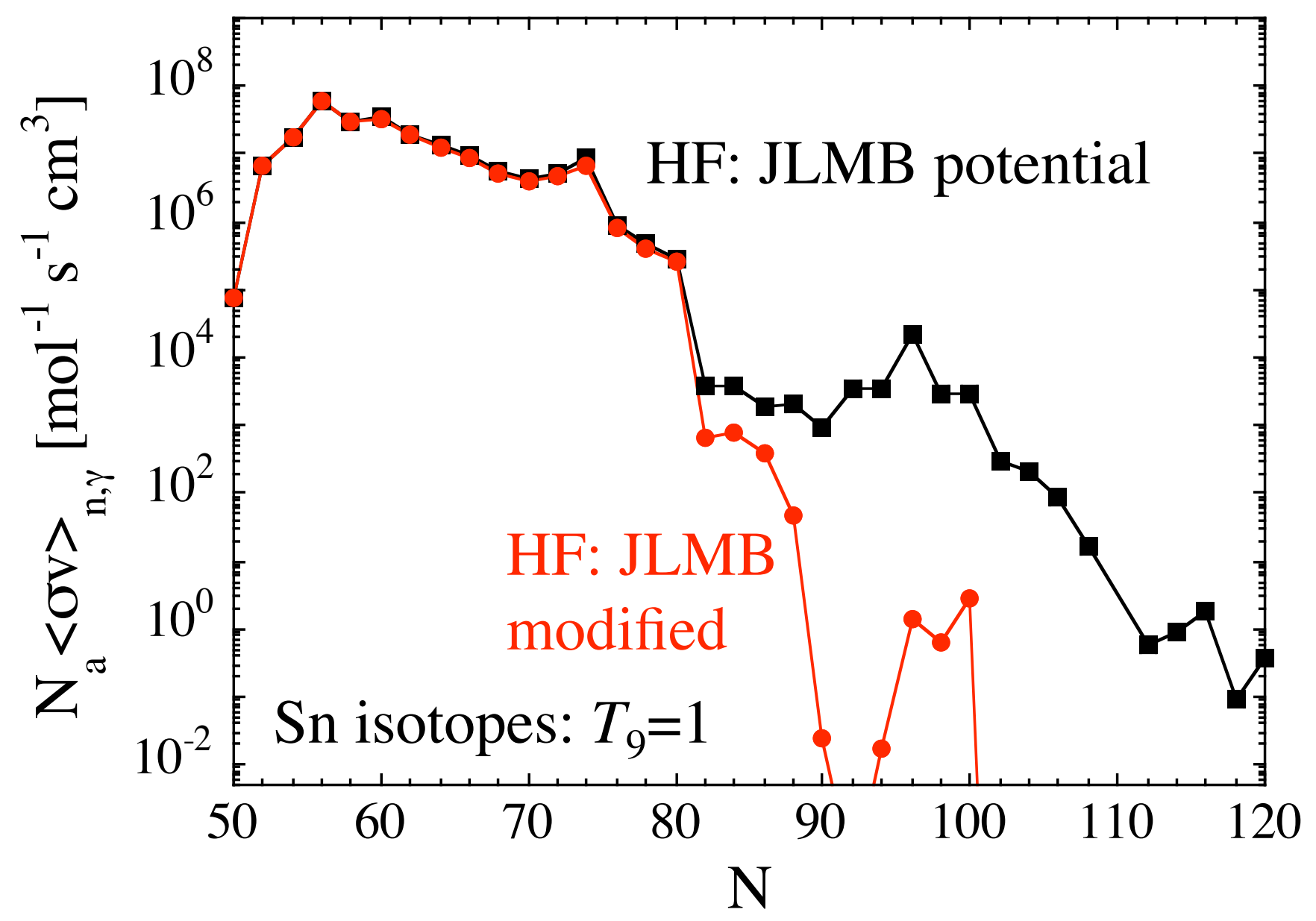




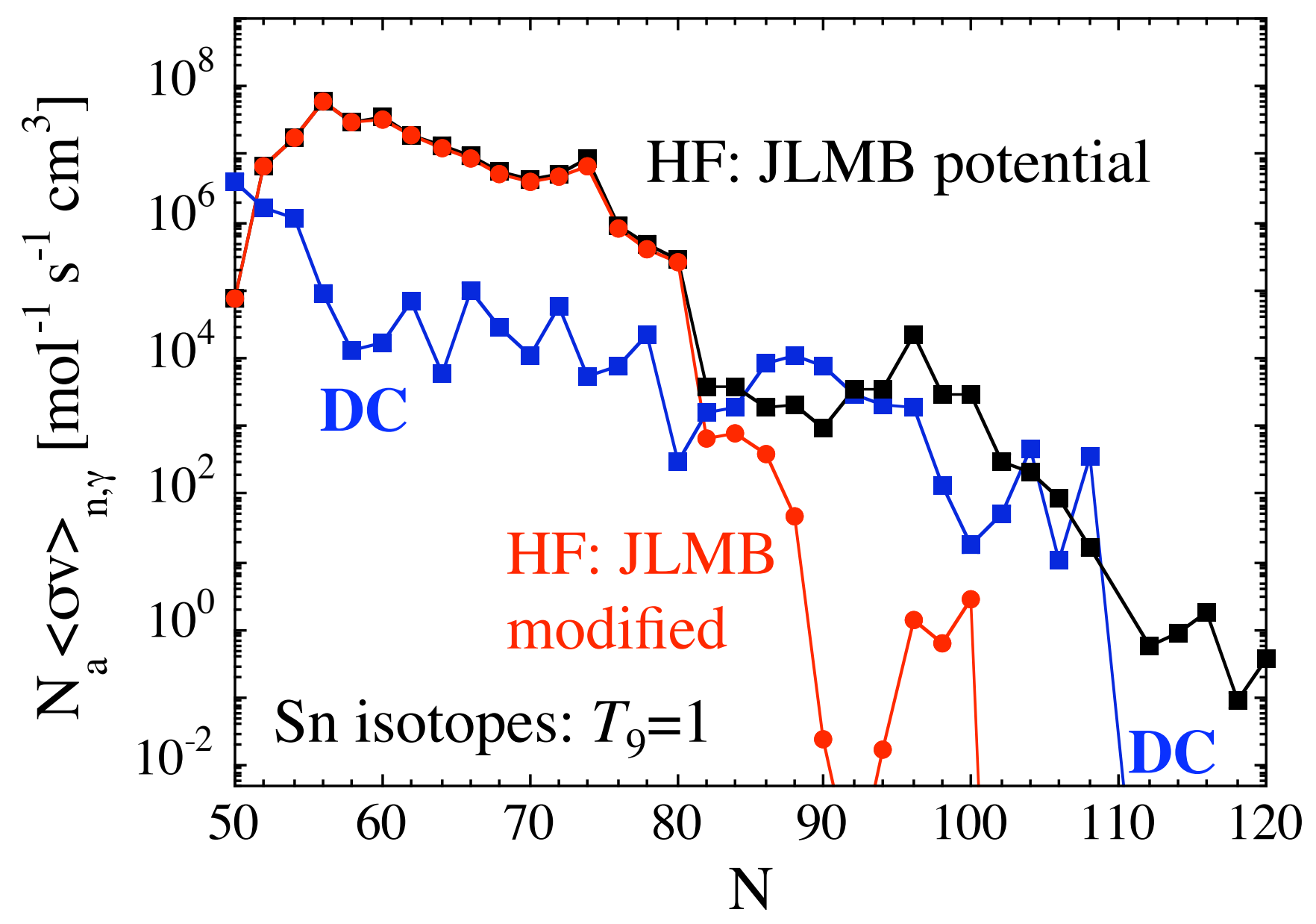




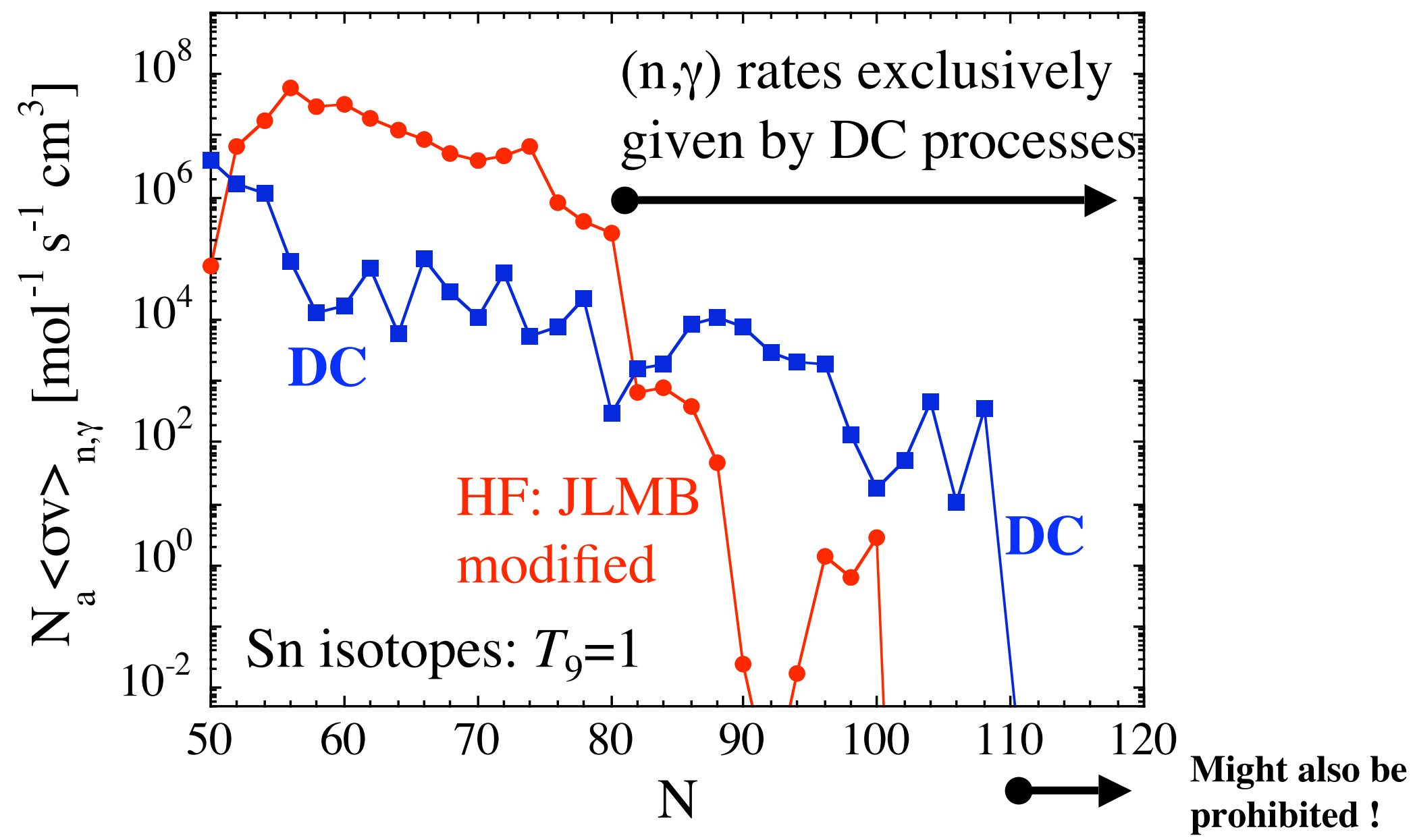

The dominant mechanism (Eq - Pre-eq - DC) responsible for n-capture of exotic n-rich nuclei remains an open question! 


\section{Comparison of the Statistical and Direct Captures}

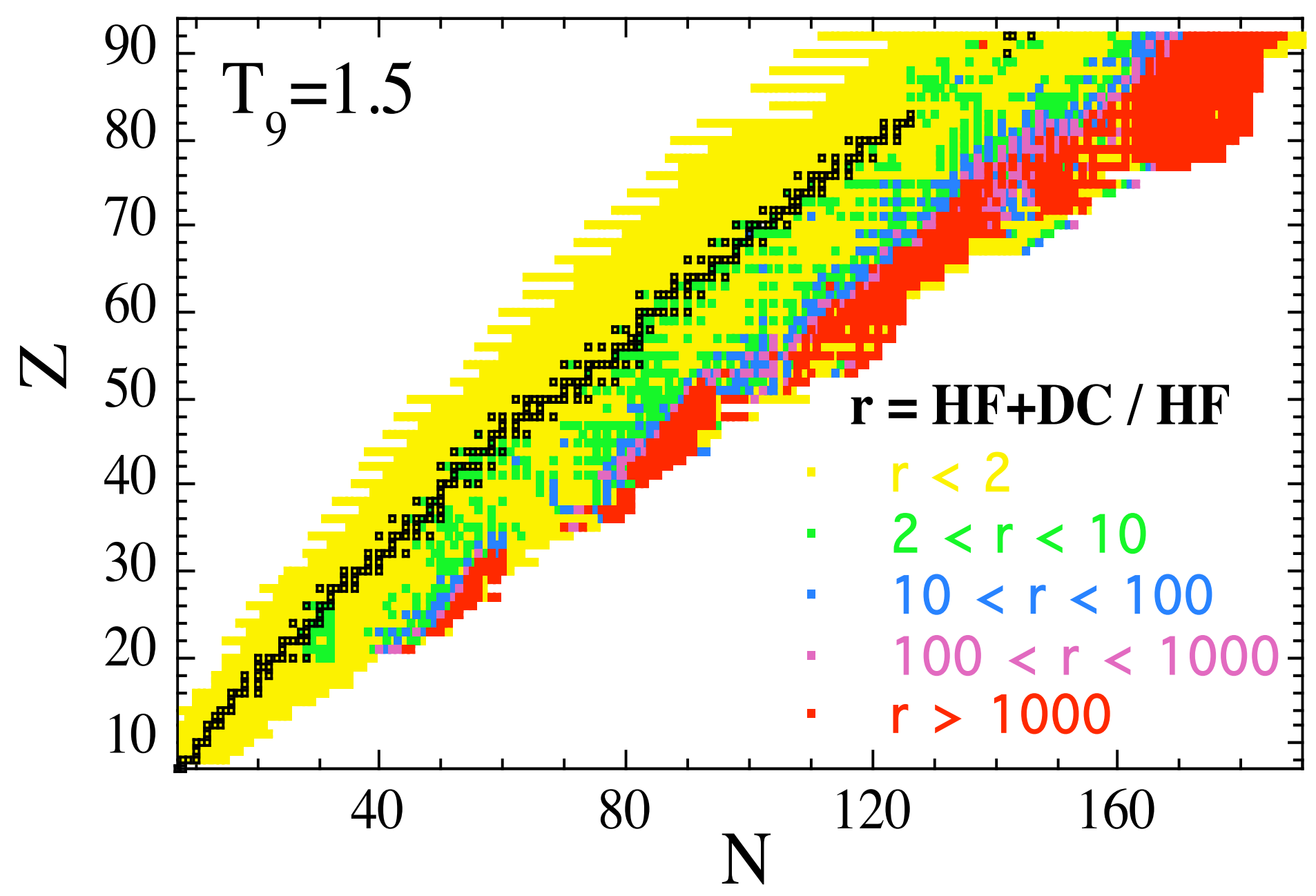




\section{Direct Captures rates}

For many nuclei with low $S_{n}$, the DC rates can become negligible: the selection rule forbids the E1-type transition to the GS or any of the available excited levels.

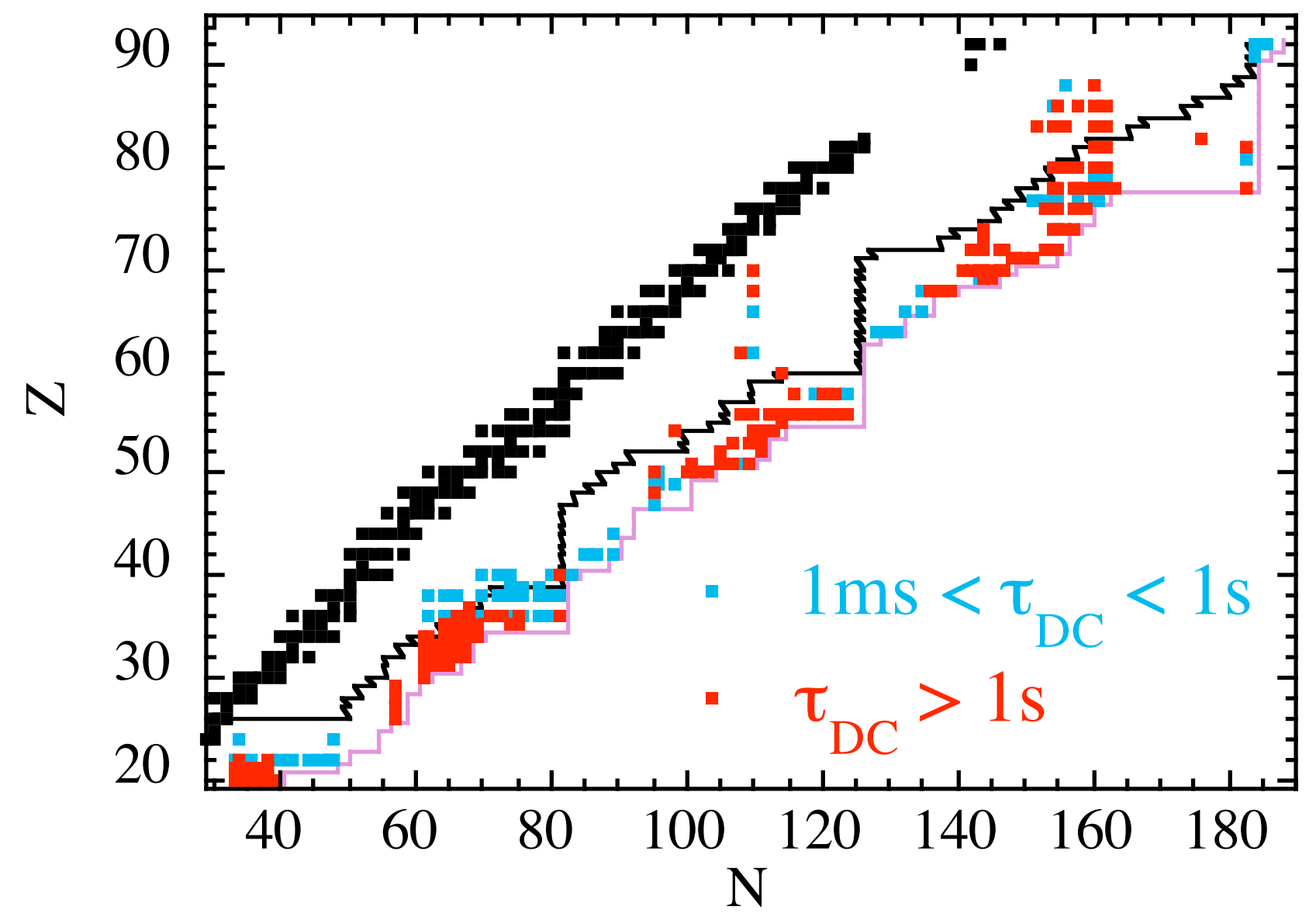

Nuclides with a half-life against neutron DC larger than 1s or ranging between $1 \mathrm{~ms}$ and $1 \mathrm{~s}$ for $\mathrm{N}_{\mathrm{n}}=10^{27} \mathrm{~cm}^{-3}$ and $\mathrm{T}_{9}=1.5$. 


\section{Nuclear Ingredients for cross section calculations}




\section{Nuclear Ingredients for HF cross section calculations}

\section{Ground \& Excited state properties}

- Ground state mass, equilibrium deformation, density distribution, shell energy, pairing energy, spl scheme, etc...

- Excited spectrum $(E, J, \pi) \quad$ - Nuclear Level Densities $\rho(E, J, \pi)$

- Partial Level Densities $\omega\left(\mathrm{p}_{\pi}, \mathrm{h}_{\pi}, \mathrm{p}_{v}, \mathrm{~h}_{v}, \mathrm{E}_{\mathrm{x}}\right)$

- Energy surfaces - Fission barrier

\section{Interaction properties}

- Nucleon-nucleus optical potential

- Alpha-nucleus interaction potential

- $\gamma$-strength function: Giant Resonance Properties

- Fission dynamics (neutron-induced, spontaneous fission)

$\longrightarrow$ Nuclear Ingredients from

(1) direct experimental data

(2) indirect (model-dep) exp. data

(3) theoretical models 


\title{
Astrophysics Applications
}

\begin{abstract}
Exotic nuclei
Many nuclei (thousands: sph-def; even-odd)

Many properties (GS, strong, electromag., weak)

Energies below the Coulomb barrier (charged-p)
\end{abstract}




\section{Challenges in nuclear models}

(at least for nuclear astrophysics)

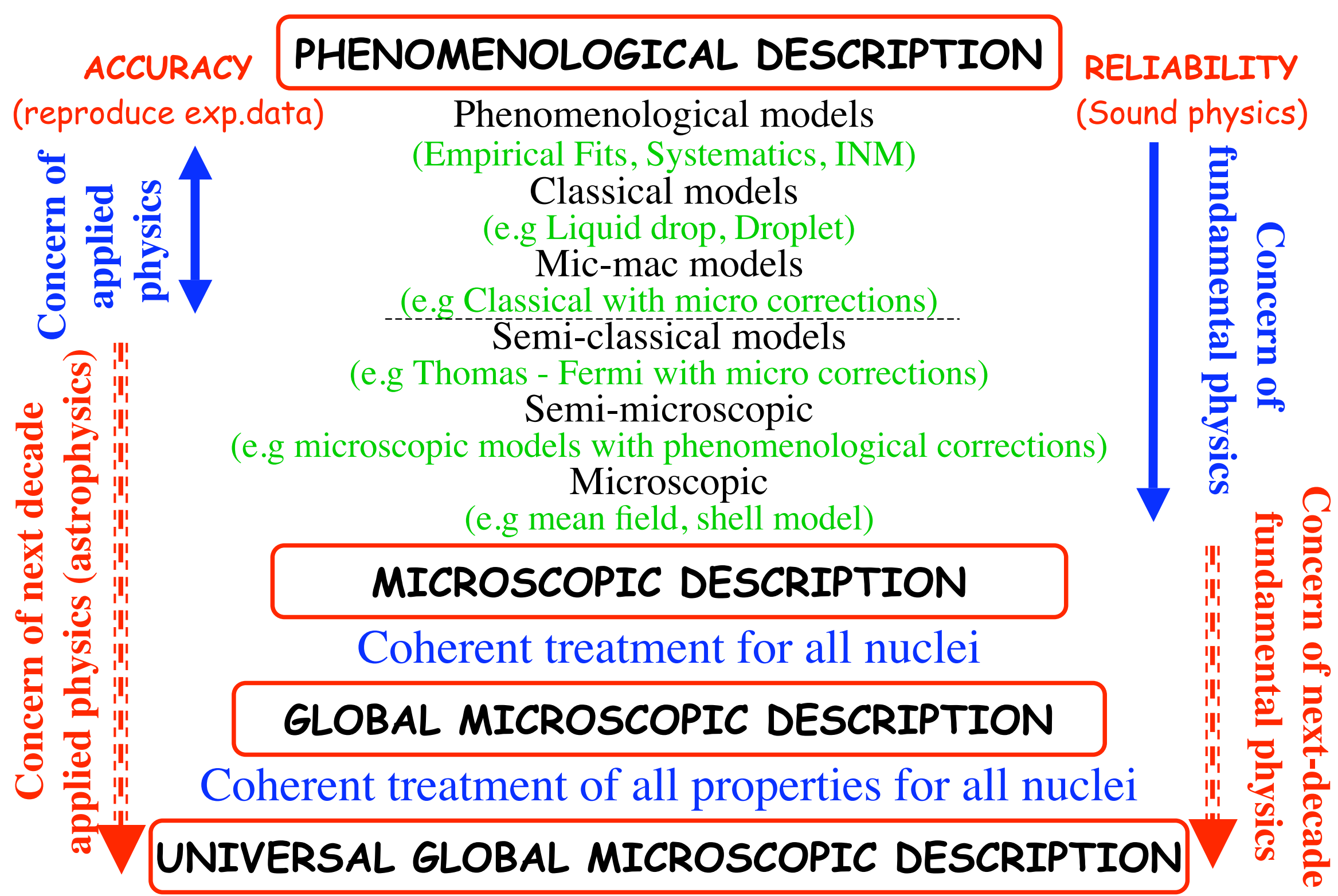


Global Approaches to Strong, Weak and Electromagnetic Interactions

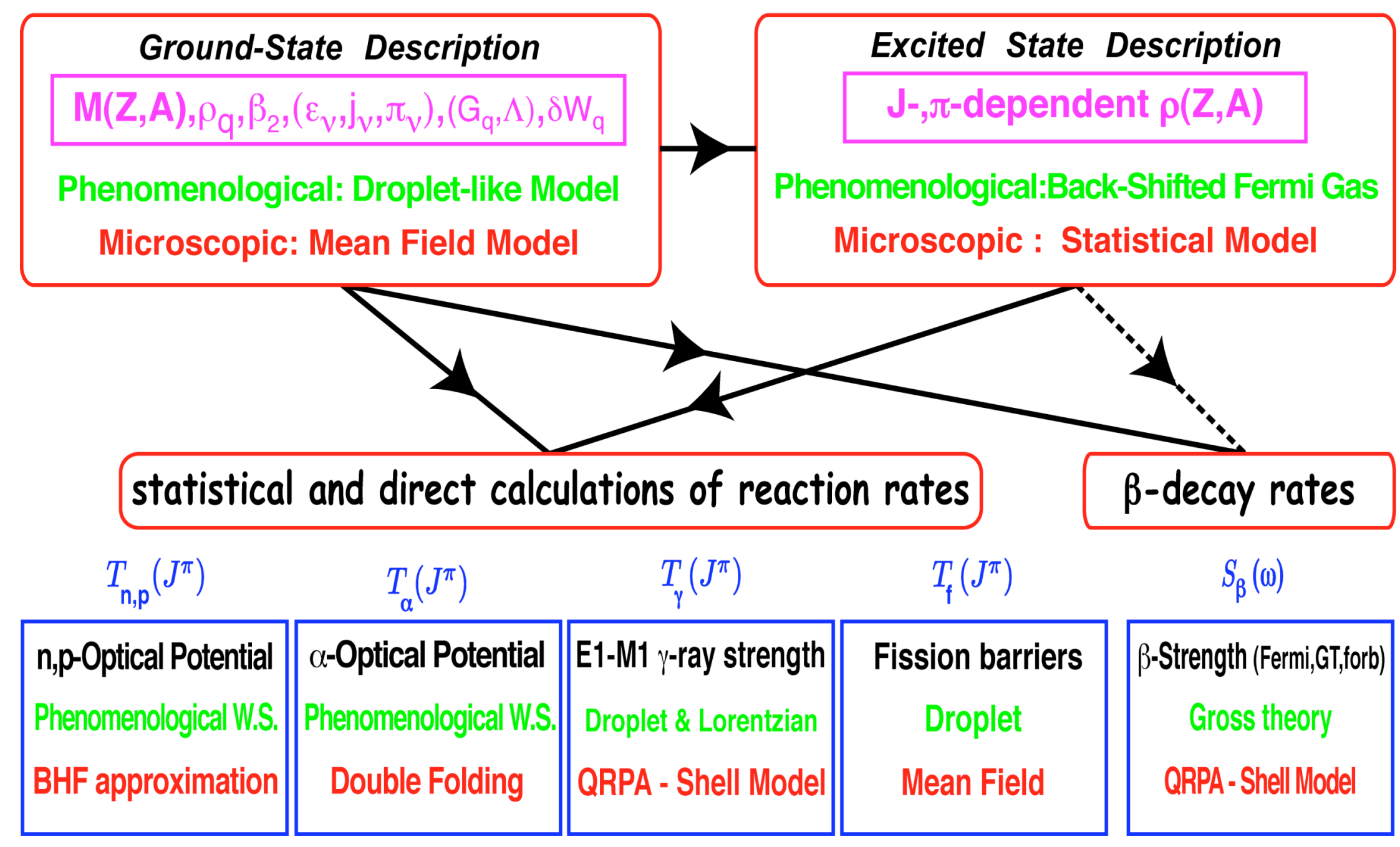

--> Still large uncertainties for exotic nuclei: $(n, \gamma)$ rates: factor $\sim 10^{2}-10^{6}$ $\beta$-decay rates: factor $\sim 10(?)$ 
Direct or indirect observables entering nuclear reaction models

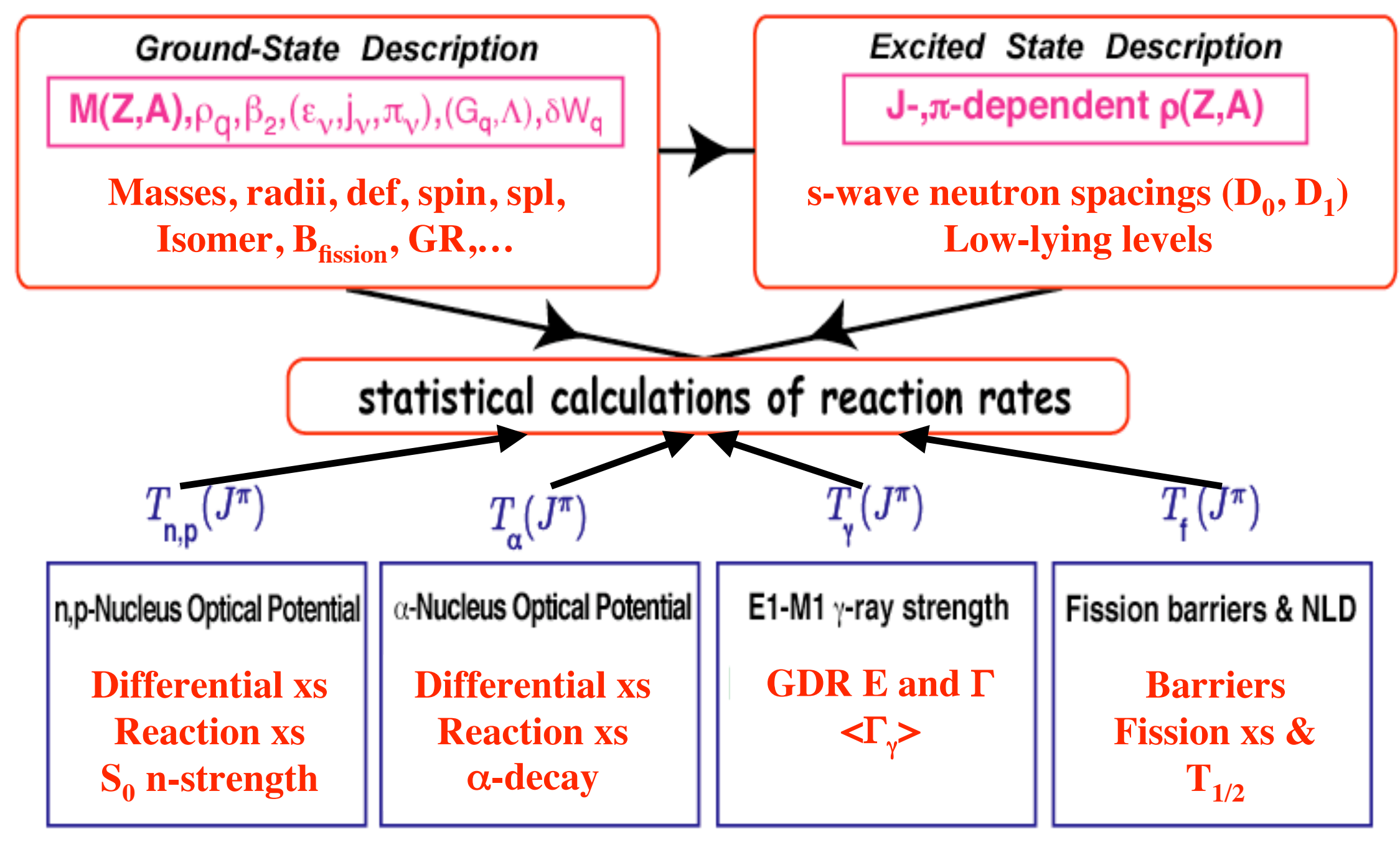


Need for a regularly updated libraries of experimental data and corresponding evaluated input parameters:

Fundamental

- for accurate cross section (and rate) calculations

- to improve systematics of phenomelogical models

- to determine the best set of parameters for theoretical models

- to test/validate global microscopic models (“accurate" mass models, NLD models, GDR models, ...)

Extension of the systematics to unstable nuclei (masses, radii, ...) Still many properties on stable nuclei are missing !! 


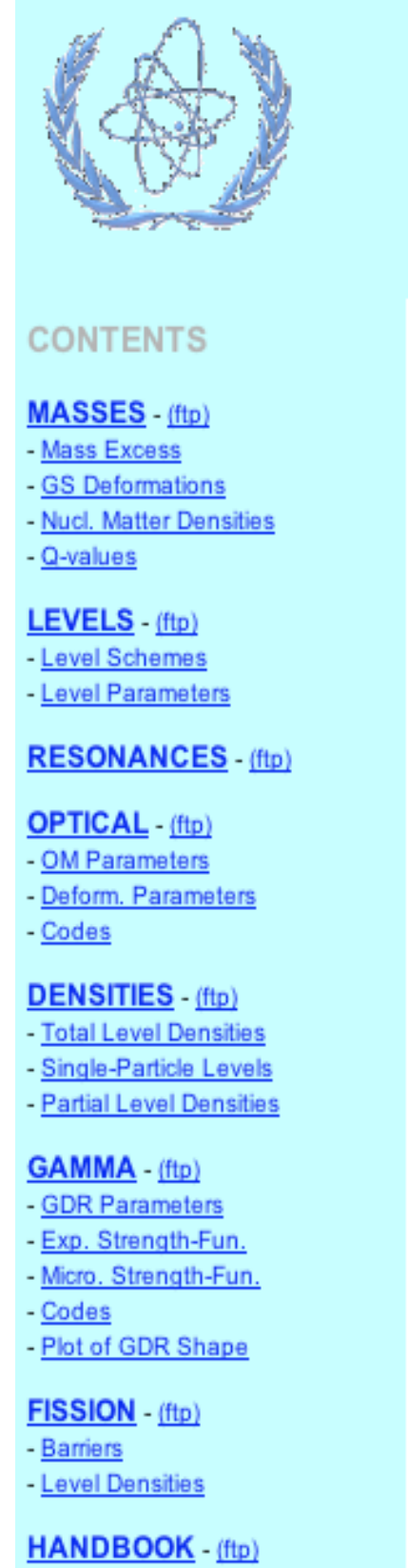

\section{Reference Input Parameter Library}

Related links: NDS-home $\underline{\text { CD-ROMs }}$ RIPL-1 ENSDF NuDat EMPIRE-II

\section{Coordinated by the IAEA Nuclear Data Section}

Release Date: April 20, 2003

RIPL-2 library contains input parameters for theoretical calculations of nuclear reactions involving light particles such as n, p, d, t, 3- $\mathrm{He}, 4-\mathrm{He}$, and gammas at incident energies up to about $100 \mathrm{MeV}$. The library contains nuclear masses, deformations, matter densities, discrete levels and decay schemes, spacings of neutron resonances, optical model potentials, level density parameters, Giant Resonance parameters, gamma-ray strength-functions, and fission barriers. It also includes extensive database of level densities, gamma-ray strength-functions and fission barriers calculated with microscopic approaches. Several computer codes are provided in order to facilitate use of the library.

RIPL-2 has been developed under an international project coordinated by the IAEA Nuclear Data Section as a continuation of the RIPL-1 project concluded in 1997. The original scope of RIPL-2 was to test and validate RIPL-1 database. In the course of work most of the recommended files were extended and many new were added. On the other hand, a number of so called 'other' files from RIPL-1 are not included in RIPL-2. Testing of these files was not at the level typical for the RIPL-2 files but they may still be a valuable source of additional information. Therefore, RIPL-1 remains available although use of the RIPL-2 data is generally recommended.

RIPL-2 data are organized into segments, which can be accessed through the Contents of RIPL-2 or through the navigation bar on the left. The (ftp) links next to segment names provide direct (ftp-like) access to the RIPL-2 directories. Entire segments (tarred and gzipped) can be downloaded by clicking on a file with a proper segment name and .tgz extension (e.g., masses.tgz). These files are placed in their respective RIPL-2 directories.

\section{Participants}
Institute of Isotope and Surface Chemistry Chemical Research Cente P.O. Box 77, H-1525 Budapest, Hungary E-mail: belgya@alpha0.iki.kfki.hu

\section{O. Bersillon}
Service de Physique et Techniques Nucleaires Centre d'Etudes Nucleaires de Bruyeres-le-Chatel B.P. 12, F-91680 Bruyeres-le-Chatel, France E-mail: olivier.bersillon@cea.fr

R. Capote Noy Centro de Estudios Aplicados al Desarollo Nuclear Calle 30 No. 502 e/5ta y $7 \mathrm{ma}$

$$
\text { Miramar, Playa }
$$

11300 Ciudad de la Habana, Cuba E-mail: rcapote@ceaden.edu.cu

Etc

\section{T. Fukahori}

Nuclear Data Center

Japan Atomic Energy Research Institute

Tokai-mura, Naka-gun

Ibaraki-ken 319-1195, Japan

E-mail: fukahori@ndc.tokai.jaeri.go.jp 


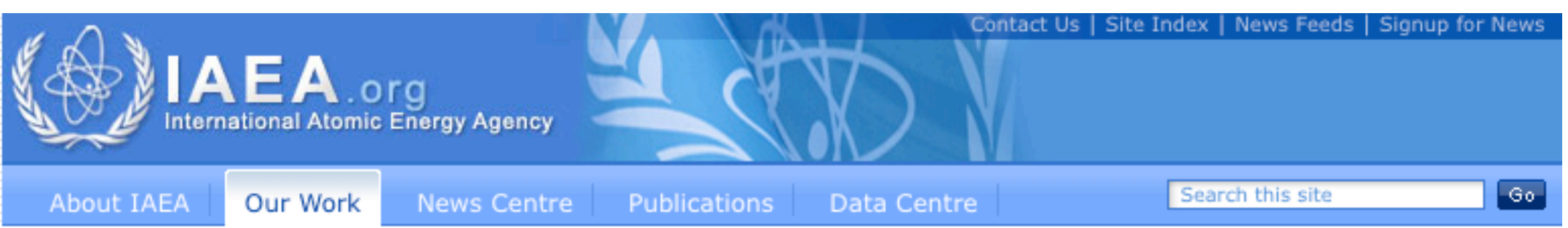

You are in: * Home * Nuclear Sciences and Applications \& Physical \& Chemical Sciences * Nuclear Data

\begin{tabular}{|c|}
\hline $\begin{array}{l}\text { Division of Physical } \\
\text { and Chemical } \\
\text { Sciences }\end{array}$ \\
\hline $\begin{array}{l}\text { Industrial Applications } \\
\text { and Chemistry }\end{array}$ \\
\hline Isotope Hydrology \\
\hline Nuclear Data \\
\hline Physics \\
\hline Hydrology Laboratory \\
\hline
\end{tabular}

\section{Nuclear Data Section}

Parameters for Calculation of Nuclear Reactions of Relevance to Energy and Non-Energy Nuclear Applications

\section{CRP on Reference Input Parameter Library: Phase III}

Please refer to the published RIPL paper:

"RIPL - Reference Input Parameter Library for Calculation of Nuclear Reactions and Nuclear Data Evaluations",

Nuclear Data Sheets 110 (2009) 3107-3214

R. Capote, M. Herman, P. Oblozinsky, P.G. Young, S. Goriely, T. Belgya, A.V.

Ignatyuk, A.J. Koning, S. Hilaire, V.A. Plujko, M. Avrigeanu, O. Bersillon, M.B.

Chadwick, T. Fukahori, Zhigang Ge, Yinlu Han, S. Kailas, J. Kopecky, V.M. Maslov,

G. Reffo, M. Sin, E.Sh. Soukhovitskii and P. Talou.

Retrieval web page will be available in January 2010 .

DATA FILES PRODUCED, 14 December 2009

- 1. Masses (Coordinator: S. Goriely)

- 2. Levels (Coordinators: T. Belgya and R.Capote)

- 3. Resonances (Coordinator: A. Ignatyuk)

- 4. Optical model (Coordinators: A. Koning and R.Capote)

- 5. Level densities (Coordinator: S. Hilaire)

- 6. Gamma-ray strength function (Coordinator: V. Plujko)

- 7. Fission (Coordinator: S. Goriely) Codes

Etc

- Codes: SCAT2000, ECIS03, OPTMAN, PFNS-LosAlamos-Mode

\section{Mission \& Role}

About Us

Coordinated Research Projects

Meetings

Publications

\section{Nuclear Data}

Services

Atomic \& Molecular

Data 


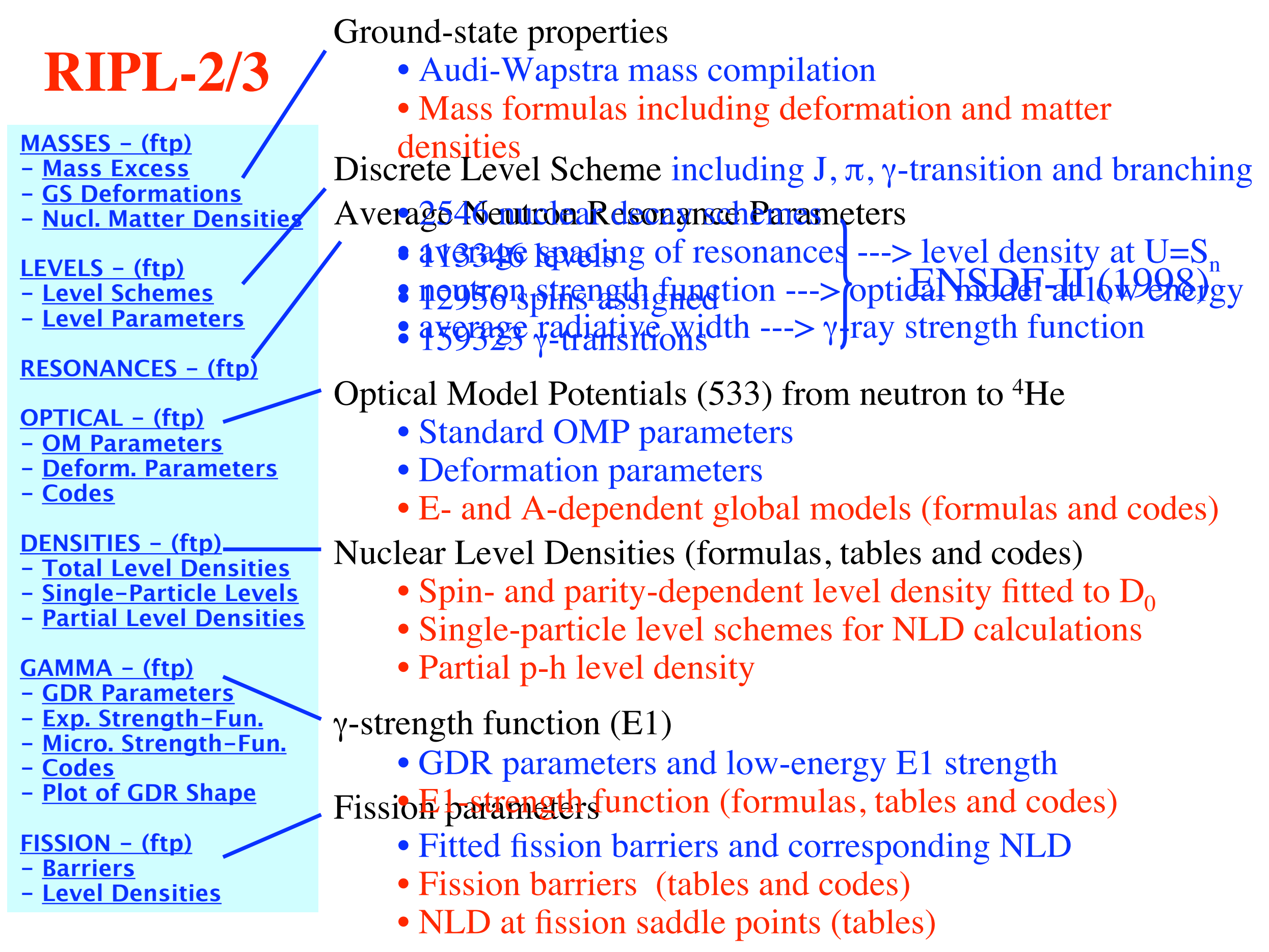


Nuclear structure properties 


\section{Global mass models}

$\underline{\text { Reliability Accuracy }}$

- Macroscopic-Microscopic Approaches

Liquid drop model (Myers \& Swiateki 1966)

Droplet model (Hilf et al. 1976)

FRDM model (Moller et al. 1995)

KUTY model (Koura et al. 2000)

- Approximation to Microscopic models

Shell model (Duflo \& Zuker 1995)

ETFSI model (Aboussir et al. 1995)

- Mean Field Model

Hartree-Fock-BCS model

Hartree-Fock-Bogolyubov model

EDF, RHB, Shell model

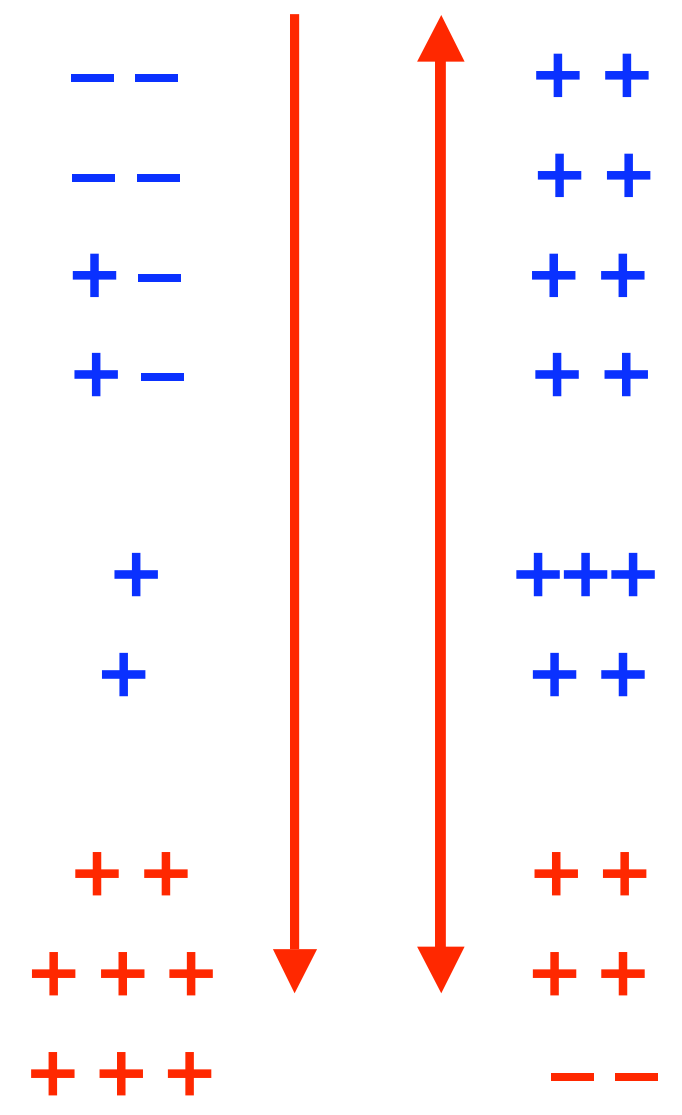

Typical deviations for the best mass formulas:

$\operatorname{rms}(M)=600-700 \mathrm{keV}$ on $2149(\mathrm{Z} \geq 8)$ experimental masses 


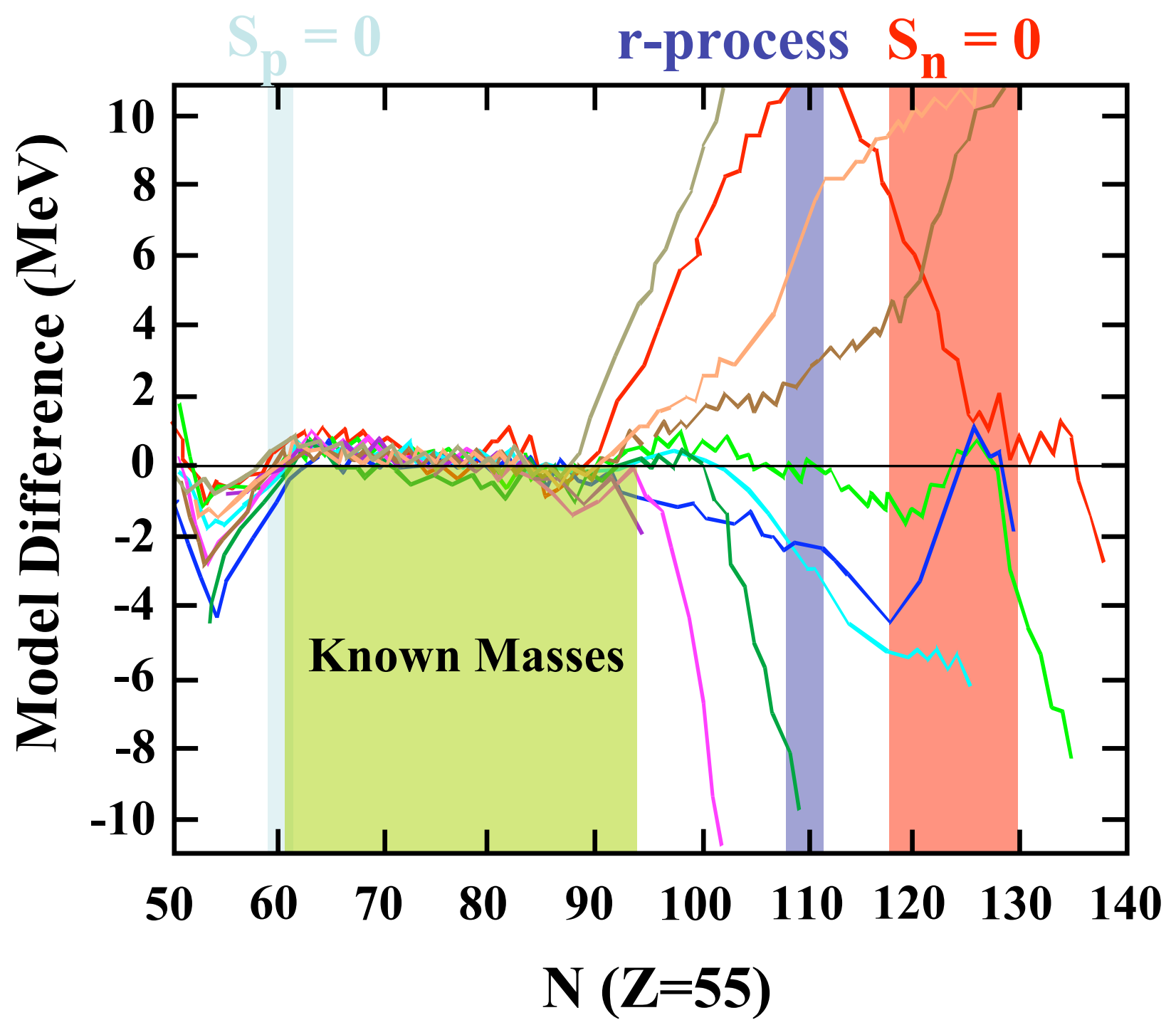


Uncertainties in the prediction of masses far away from the experimentally known region

Two identical "droplet models" but with two different parametrizations

Hilf et al. (1976) versus von Groote et al. (1976)

rms deviation on exp masses $670 \mathrm{keV}$ (1976 data) - $950 \mathrm{keV}$ (2003 data)

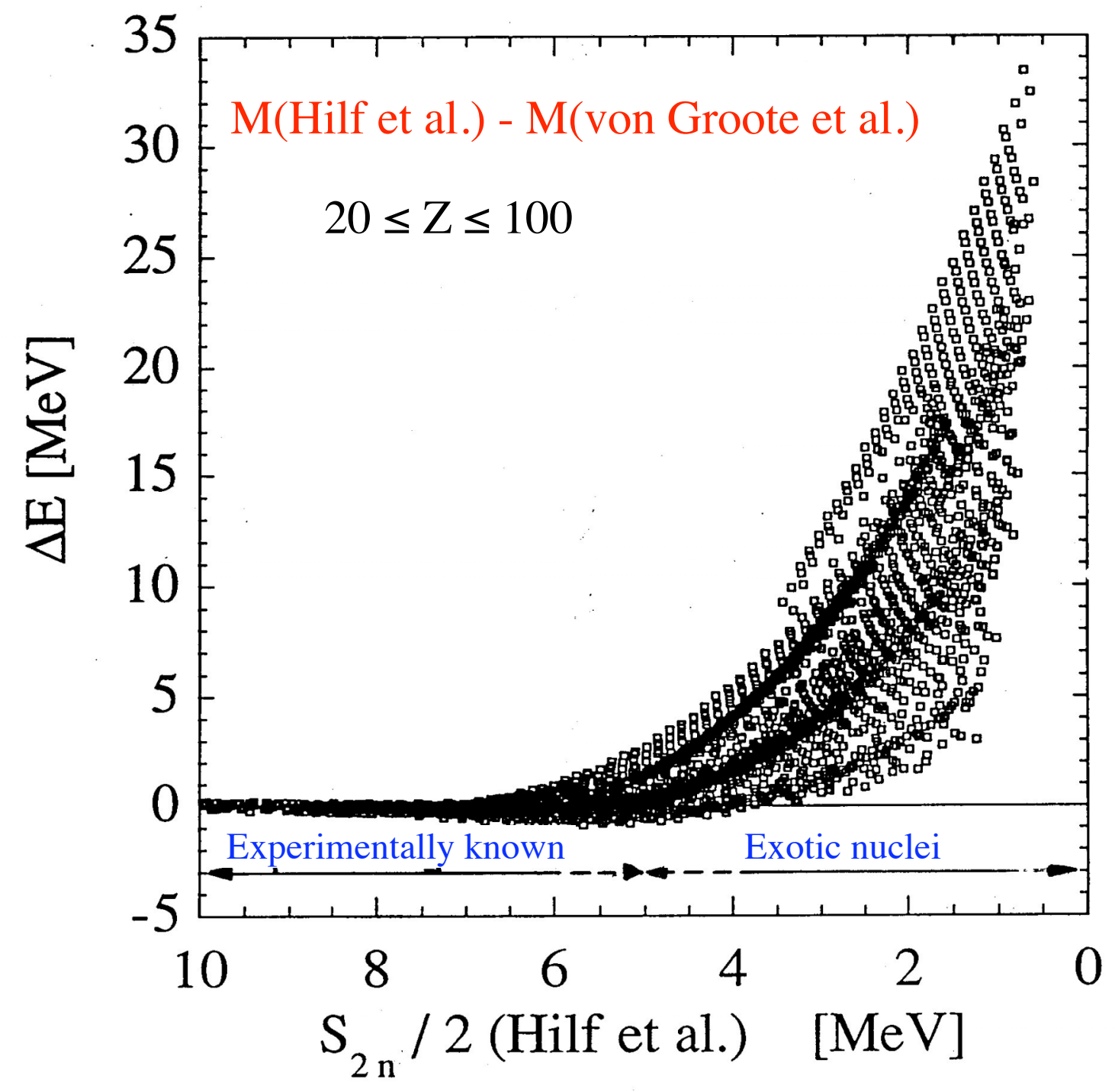




\section{Nuclear mass models}

Nuclear mass models provide all basic nuclear ingredients:

Mass excess (Q-values), deformation, GS spin and parity

but also single-particle levels, pairing strength, density distributions, ... in the GS as well as non-equilibrium (e.g fission path) configuration

Building blocks for the prediction of ingredients of relevance in the determination of nuclear reaction rates and $\beta$-decay rates, such as

- nuclear level densities

- $\gamma$-ray strengths

- fission probabilities

- etc ...

as well as for the nuclear/neutron matter Equation of State (NEUTRON STARS)

The criteria to qualify a mass model should NOT be restricted to the rms deviation wrt to exp. masses, but also include (in particular when universality is aimed at)

- the quality of the underlying physics (sound, coherent, "microscopic",...)

- all the observables of relevance in the specific (astrophysics) applications 


\section{Observables considered}

- 2149 experimental masses from Audi et al. (2003)

- 782 exp. charge radii, calculated at each iteration

- Symmetric nuclear matter properties

- $m^{*} \sim 0.6-0.8$ (BHF, GQR) \& $m^{*}{ }_{\mathrm{n}}(\beta)>m_{\mathrm{p}}{ }_{\mathrm{p}}(\beta)$

- $K \sim 230$ - $240 \mathrm{MeV}$ (breathing mode)

- $E_{p o t}$ from BHF calc. \& in $4(S, T)$ channels

- Landau parameters $F_{0}(S, T), F_{1}(S, T)$

- stability condition: $F_{l}{ }^{S T}>-(2 l+1)$

- empirical $g_{0} \sim 0 ; g_{0} \sim 0.9-1.2$

- sum rules $S_{1} \sim 0 ; S_{2} \sim 0$

- Pairing gap (with/out medium effects)

-Neutron matter properties

- $J \sim 29-32 \mathrm{MeV}$

- $E_{n} / A$ from realistic BHF-like calculations

- Pairing gap

- Stability of neutron matter at all polarizations

-Giant resonances

- ISGMR, IVGDR, ISGQR

-Additional properties

- Nuclear Level Density (pairing-sensitive)

- Isomers \& Fission barriers (scan large deformations)

- Properties of the lowest 2+ levels (519 e-e nuclei)

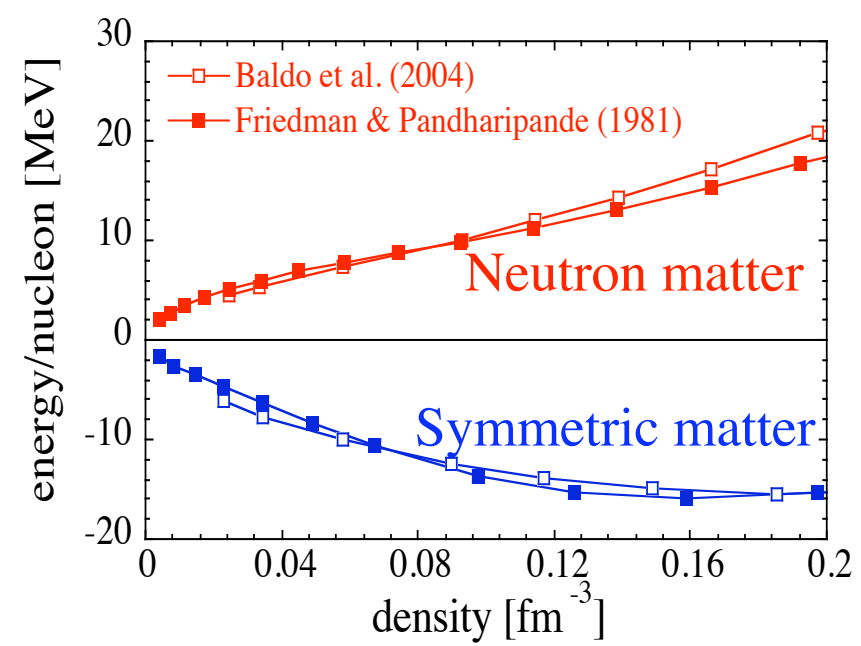

- Moment of inertia in superfluid nuclei (backbending)

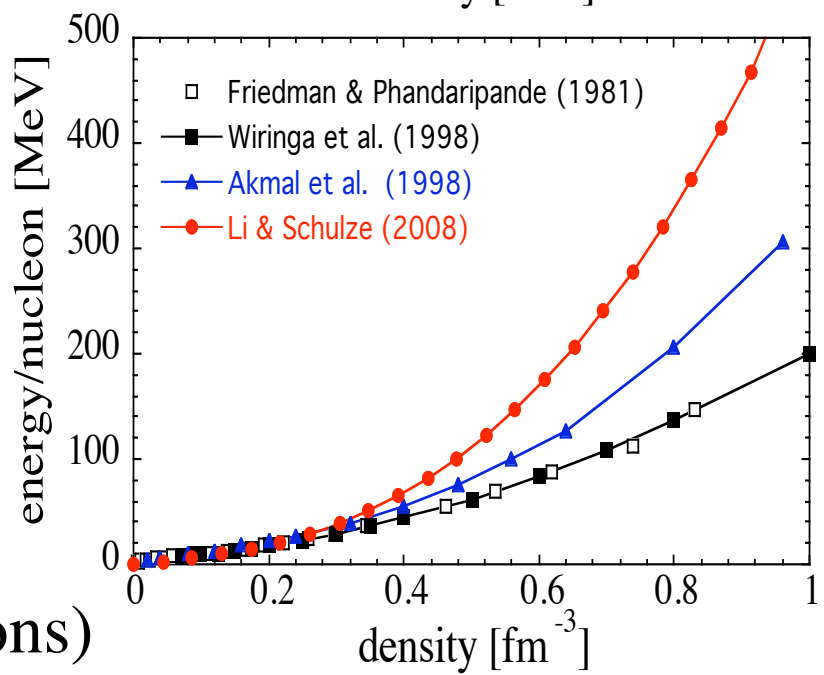




\section{HFB mass model}

\section{Adjustement of a Skyrme force to all (2149) experimental masses within the Hartree-Fock-Bogolyubov approach}

Conventional Skyrme force (10 p.) and $\delta$-pairing force $(4$ p.) to reproduce exp. masses

$$
\operatorname{rms}(\mathrm{M})=600-750 \mathrm{keV} \text { on } 2149(\mathrm{Z} \geq 8) \text { experimental masses (Audi et al, 2003) }
$$

To be compared with

- FRDM predictions: $\operatorname{rms}(\mathrm{M})=676 \mathrm{keV}(2149 \mathrm{Z} \geq 8$ nuclei $)$

- Previous HF predictions:

Traditional Skyrme forces: rms(M) >> $2 \mathrm{MeV}(120$ e-e sph)

Ex. Oak Ridge "Mass Table" based on HFB with SLy4

$$
\operatorname{rms}(\mathrm{M})=4.7 \mathrm{MeV} \text { on } 570 \text { e-e sph+def nuclei }
$$

Still some corrections that are not treated microscopically

-Wigner correction for $\mathrm{N} \sim \mathrm{Z}$ nuclei

- treatment of odd nuclei (blocking --> renormalization of pairing)

- Collective corrections (rot-vib): cranking model 


\section{Hartree-Fock-Bogolyubov model predictions}

The long road in the HFB mass model development

HFB-1-2 : $\quad$ Possible to fit all 2149 exp masses $Z \geq 8$

$\sigma_{\text {rms }}(2149$ nuc)

HFB-3:

Volume versus surface pairing

$659 \mathrm{keV}$

HFB-4-5:

Nuclear matter EoS: $M^{*}=0.92$

$635 \mathrm{keV}$

HFB-6-7:

Nuclear matter EoS: $M^{*}=0.80$

HFB-8:

Introduction of number projection

HFB-9:

Neutron matter EoS - $J=30 \mathrm{MeV}$

$660 \mathrm{keV}$

$657 \mathrm{keV}$

HFB-10-13: Low pairing \& NLD

$635 \mathrm{keV}$

$733 \mathrm{keV}$

$717 \mathrm{keV} \sim$

HFB-14:

Collective correction and Fission $B_{f}$

$729 \mathrm{keV} \sim$

HFB-15: Including Coulomb Correlations

HFB-16: with Neutron Matter pairing

$678 \mathrm{keV}$

HFB-17:

with Neutron \& Nuclear Matter pairing

$632 \mathrm{keV}$

$581 \mathrm{keV}$

HFB-18-21:

Non-Std Skyrme ( $\mathrm{t}_{4}-\mathrm{t}_{5}$ terms) - Fully stable

$577 \mathrm{keV}$

$\rightarrow$ Maximum Constraints on both Nuclei and Infinite Nuclear Matter But also fission barriers, shape isomers, NLD, GR 


\section{HFB18-21: Stiffness of the neutron matter energy density}

Low-density regime

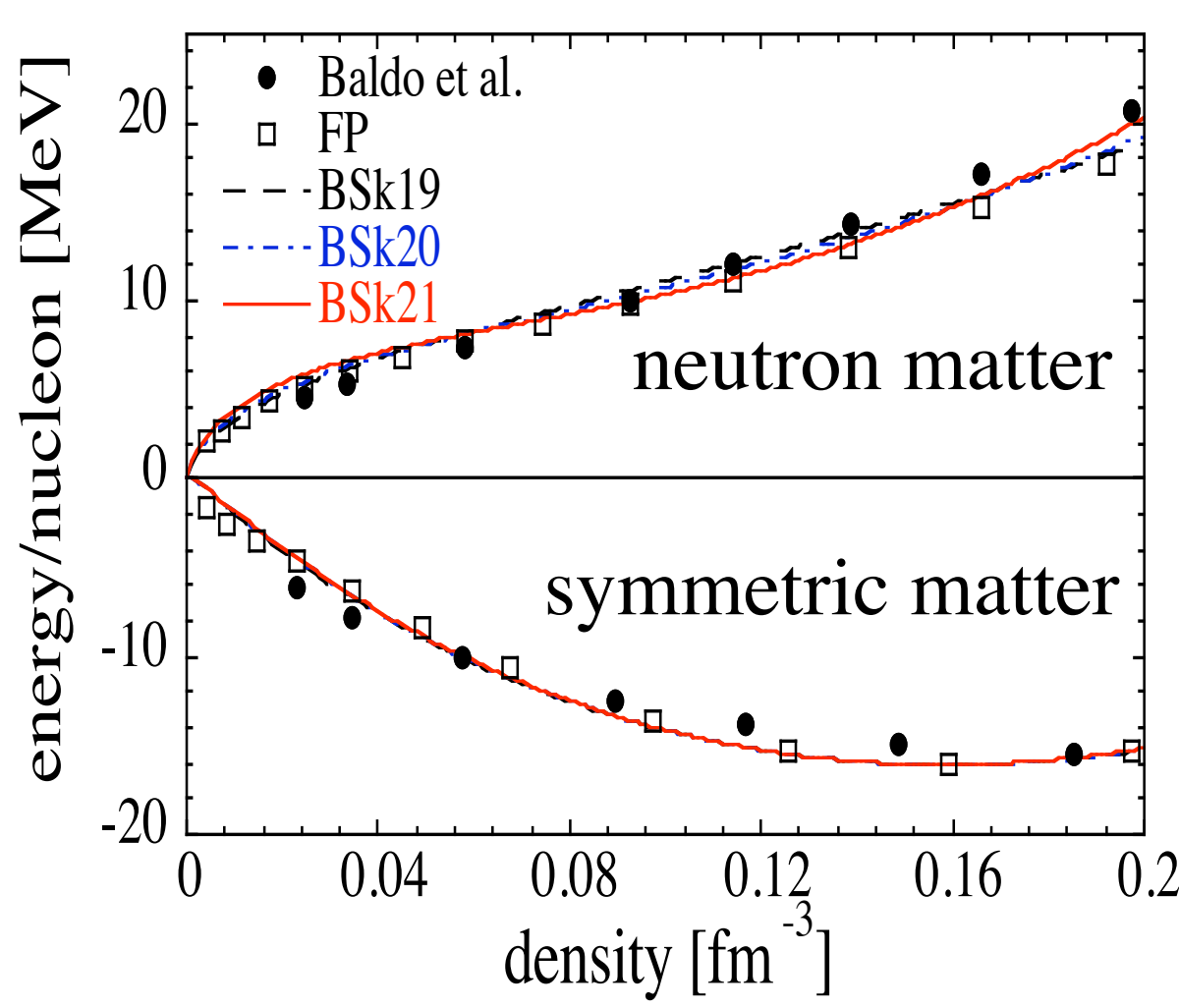

High-density regime

(neutron matter)

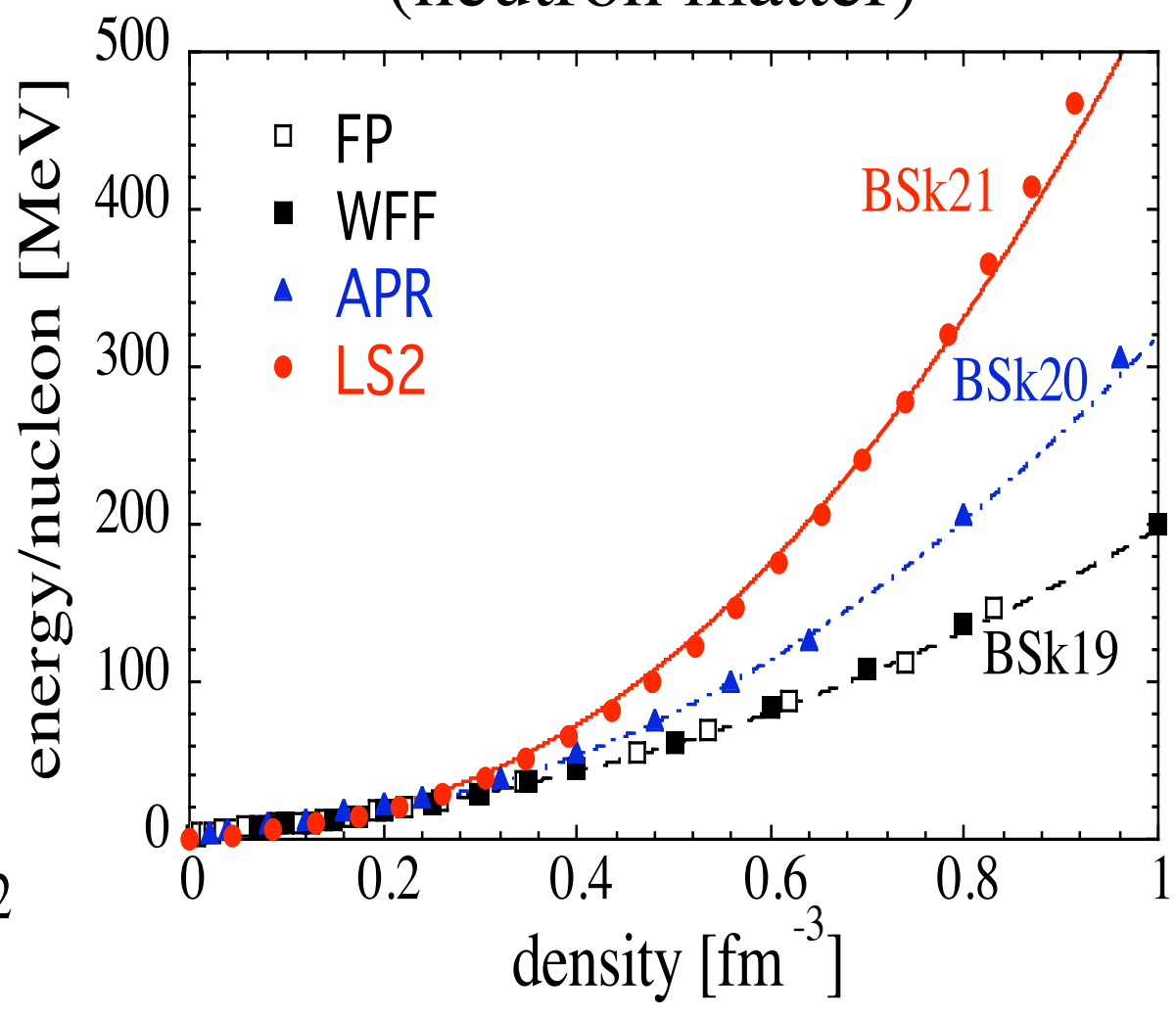




\section{Comparison with experimental masses}

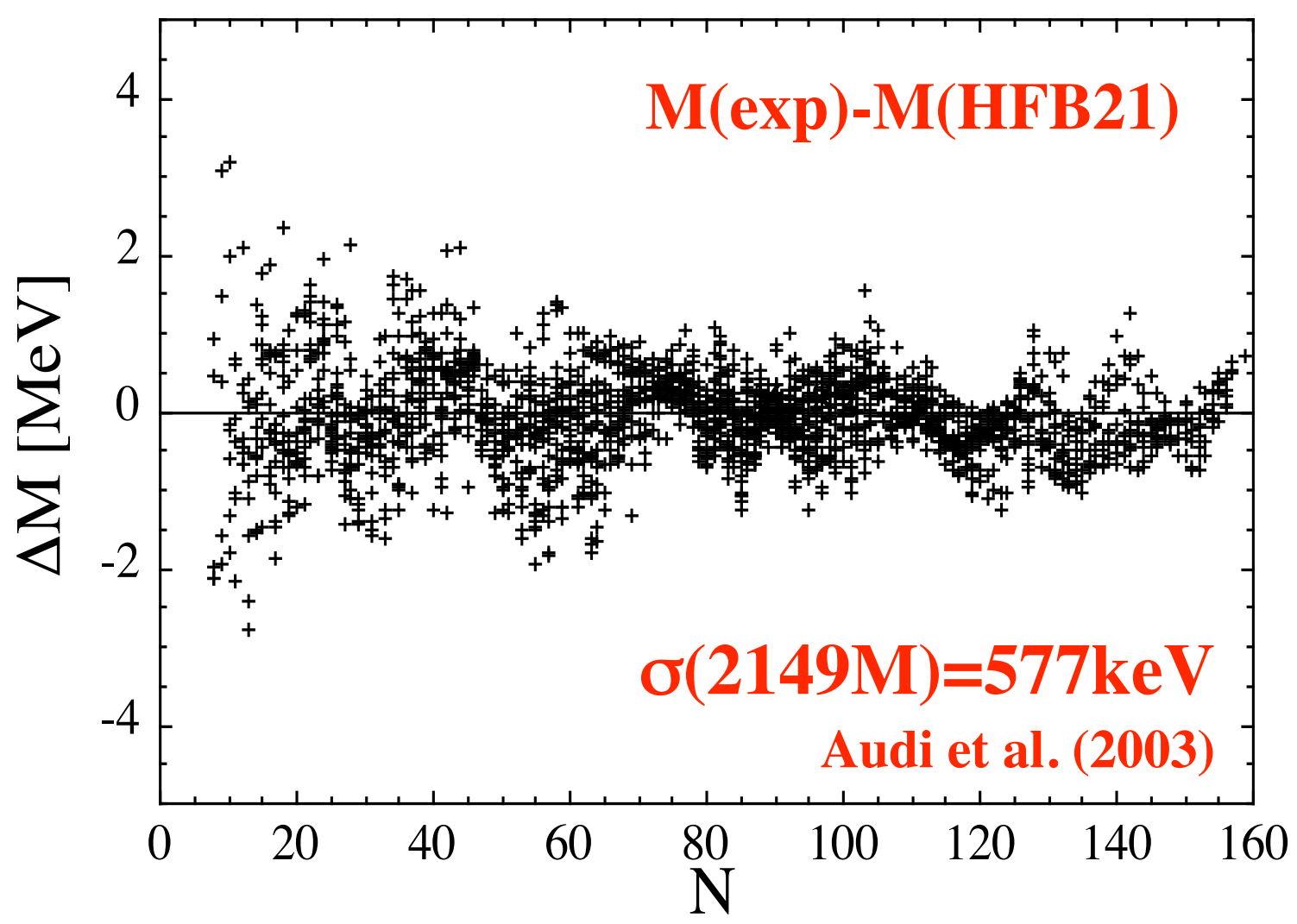

434 masses (36 $\leq \mathrm{Z} \leq 85$, p-rich) at GSI (2005)

119 masses $(28 \leq Z \leq 46$, n-rich) at JYFLTRAP (2009)
$\sigma(H F B 20) \quad \sigma(H F B 21) \quad \sigma($ FRDM $)$ $397 \mathrm{keV} \quad 388 \mathrm{keV} \quad 429 \mathrm{keV}$ $453 \mathrm{keV} \quad 625 \mathrm{keV} \quad 694 \mathrm{keV}$ 
Some examples for nuclear structure properties of interest for applications

Charge radii for 782 nuclei

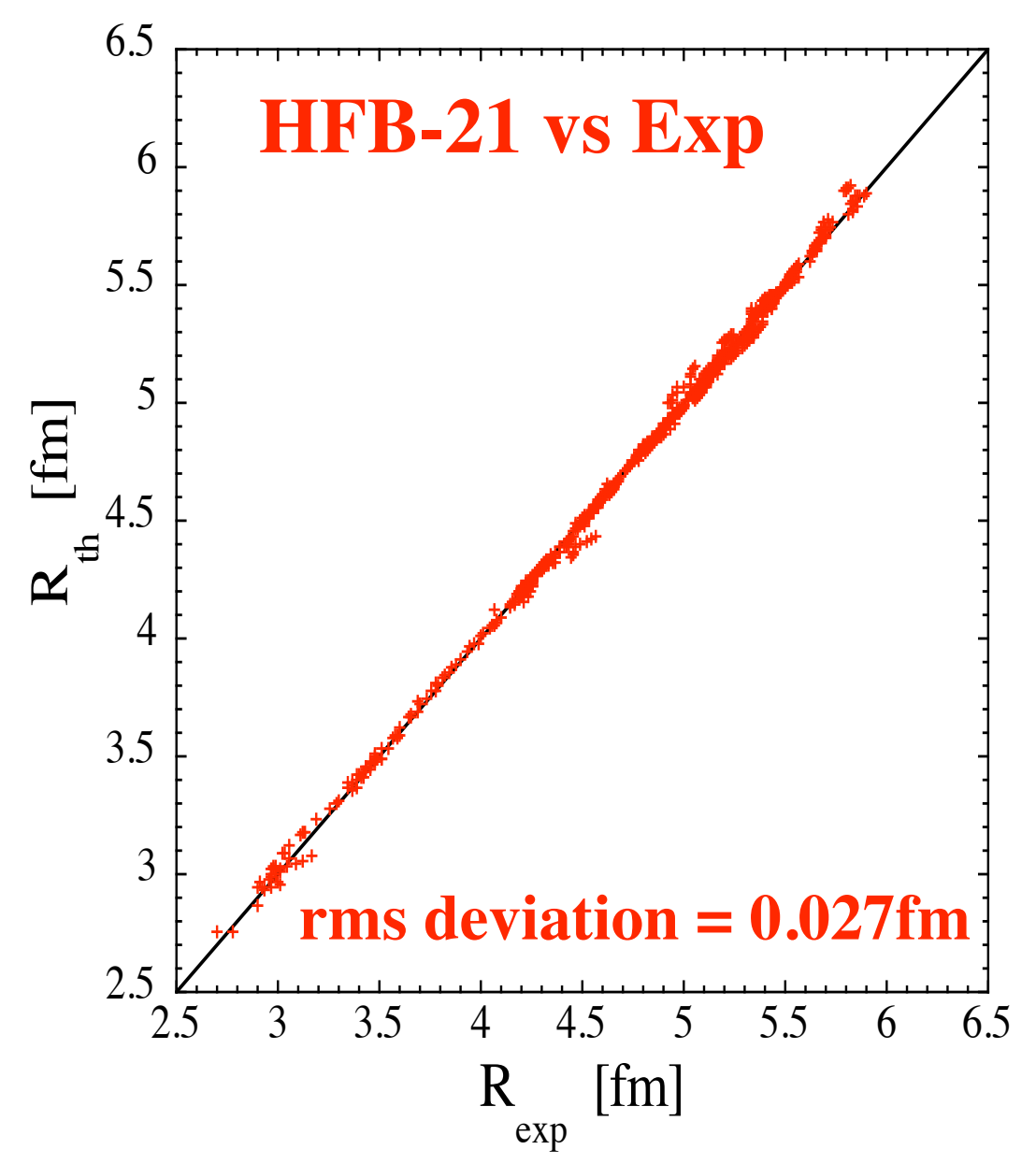

Charge distribution of ${ }^{208} \mathrm{~Pb}$

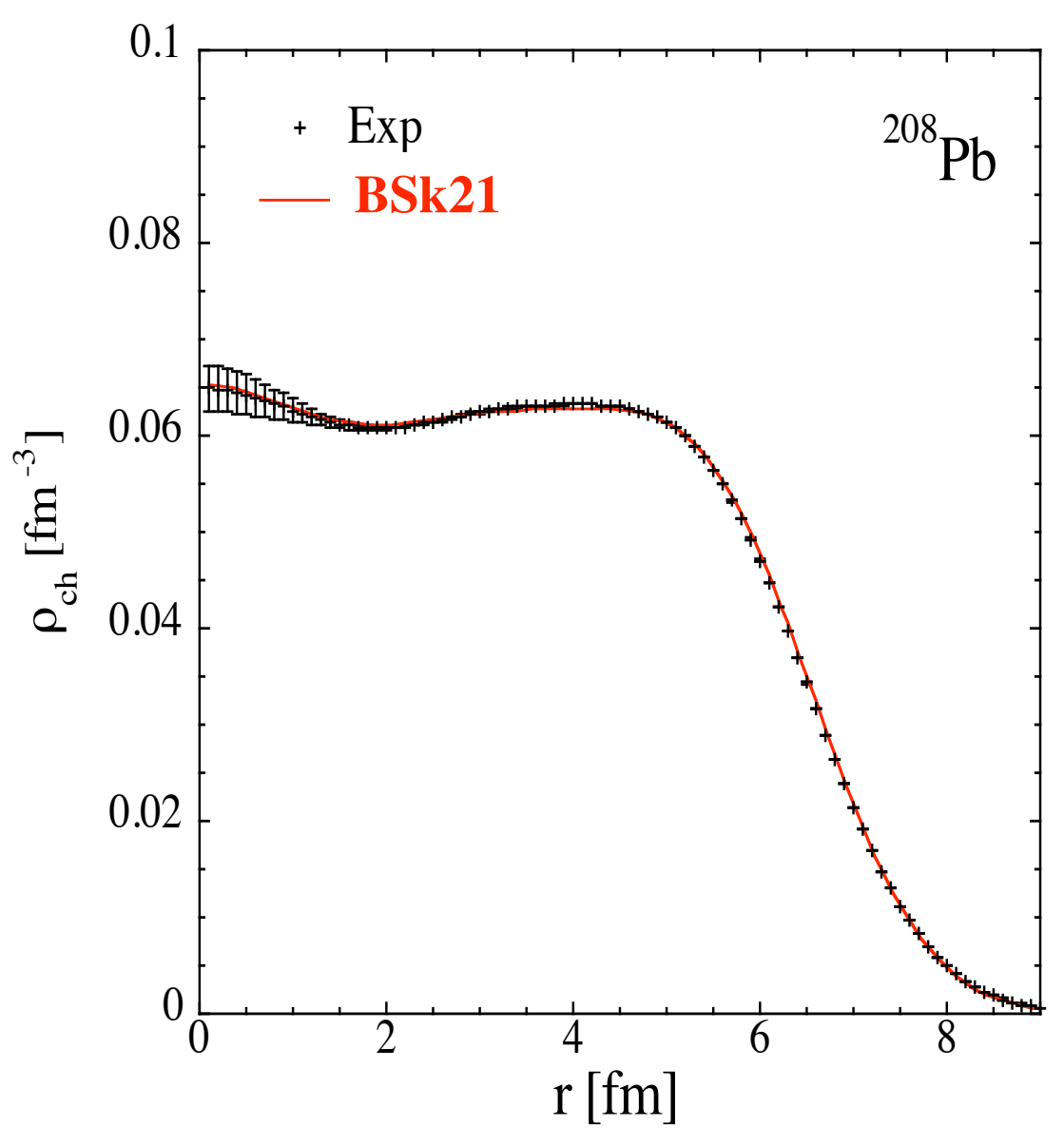


Prediction of GS spins and parities from the single-particle level scheme in the simple "last-filled orbit" approximation

For odd-A nuclei

Spherical nuclei $\left(\beta_{2} \leq 0.05\right)$ : $91 \%$ (82/90) spins correctly predicted

Deformed nuclei $\left(\beta_{2}>0.16\right)$ : 41\% (294/717) spins correctly predicted

For all odd-A and odd-odd nuclei (using Nordheim's rule)

Total of $\mathbf{1 5 8 2}$ nuclei (experimental $J^{\pi}$ from RIPL-3 database)

Spherical spl scheme for $\beta_{2} \leq 0.16$

Deformed spl scheme for $\beta_{2}>0.16$

$47 \%(740 / 1582)$ spins correctly predicted

$72 \%(1138 / 1582)$ parities correctly predicted

Full HFB-17-21 mass tables including predicted GS

$J^{\pi}$ for 8508 nuclei with $8 \leq \mathrm{Z} \leq 110$ 
$\mathrm{S}_{2 \mathrm{n}}$ surfaces from microscopic calculations affected by numerical noise (resolution of Schrodinger equations, determination of equilibrium deformation, optimized wave function, perturbative rotational correction, ...)
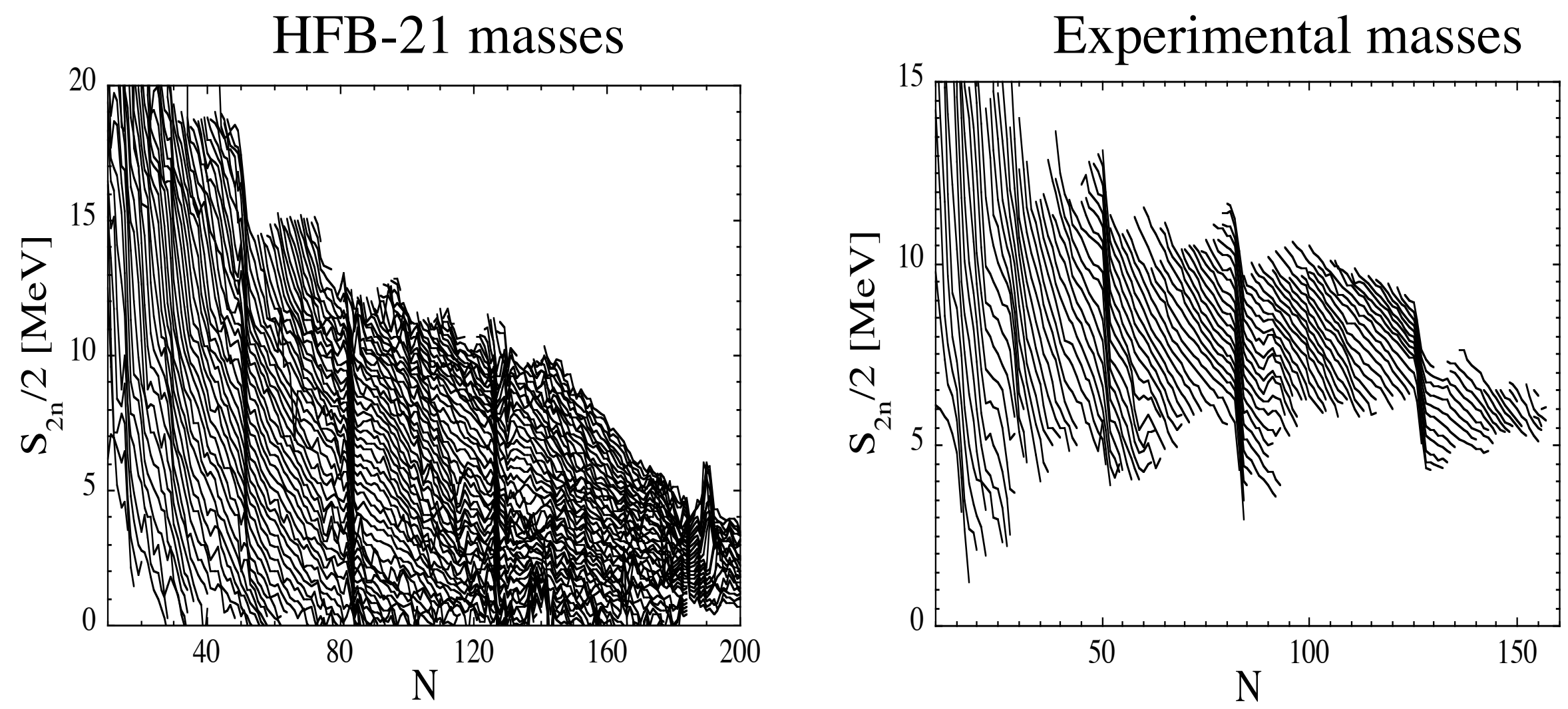

for practical applications, the mass surface should preferentially be smoothed ... but without affecting the underlying physics 


\section{Garvey-Kelson relations between nuclear masses}

$\mathbf{Z}$

The GK relations take advantage of the cancellation to first order of the most important interactions

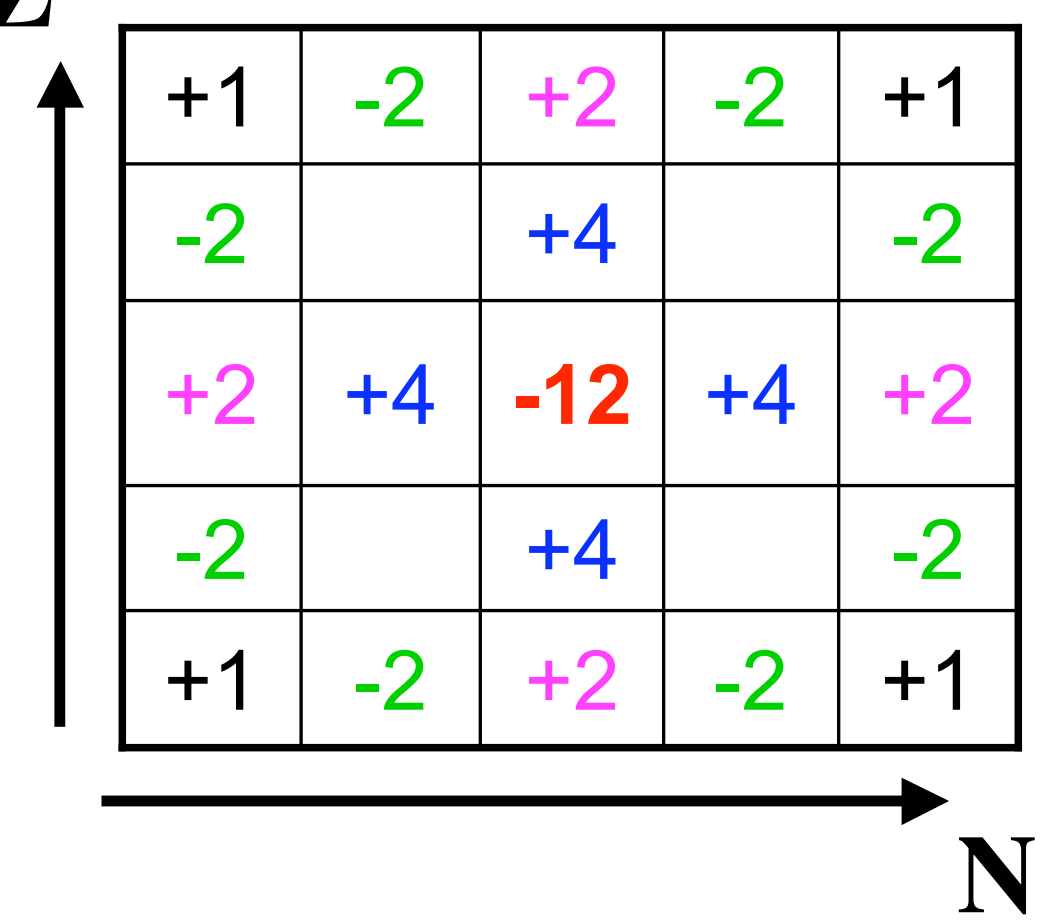

--> possibility to use an iterative procedure based on GK relations to correct the masses at iteration $i$ from the masses at iteration $i-1$, i.e to smooth the mass surface i.e to filter model noise

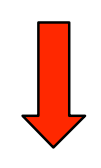

21-mass relation verified for exp. masses with an rms accuracy $\sim 90 \mathrm{keV}$

$$
\begin{aligned}
& M_{i}(Z, N)= \\
& \frac{1}{12}\left[M_{i-1}(Z+2, N-2)+M_{i-1}(Z+2, N+2)\right. \\
& +M_{i}(Z-2, N-2)+M_{i}(Z-2, N+2) \\
& -2 M_{i-1}(Z+2, N-1)-2 M_{i-1}(Z+2, N+1) \\
& -2 M_{i-1}(Z+1, N-2)-2 M_{i-1}(Z+1, N+2) \\
& -2 M_{i}(Z-1, N-2)-2 M_{i}(Z-1, N+2) \\
& -2 M_{i}(Z-2, N-1)-2 M_{i}(Z-2, N+1) \\
& +2 M_{i-1}(Z, N+2)+2 M_{i-1}(Z+2, N) \\
& +2 M_{i}(Z, N-2)+2 M_{i}(Z-2, N) \\
& +4 M_{i-1}(Z, N+1)+4 M_{i-1}(Z+1, N) \\
& \left.+4 M_{i}(Z, N-1)+4 M_{i}(Z-1, N)\right]
\end{aligned}
$$


Smoothing of the HFB masses on the basis of the GK relations (independent of experimental masses)

HFB-21

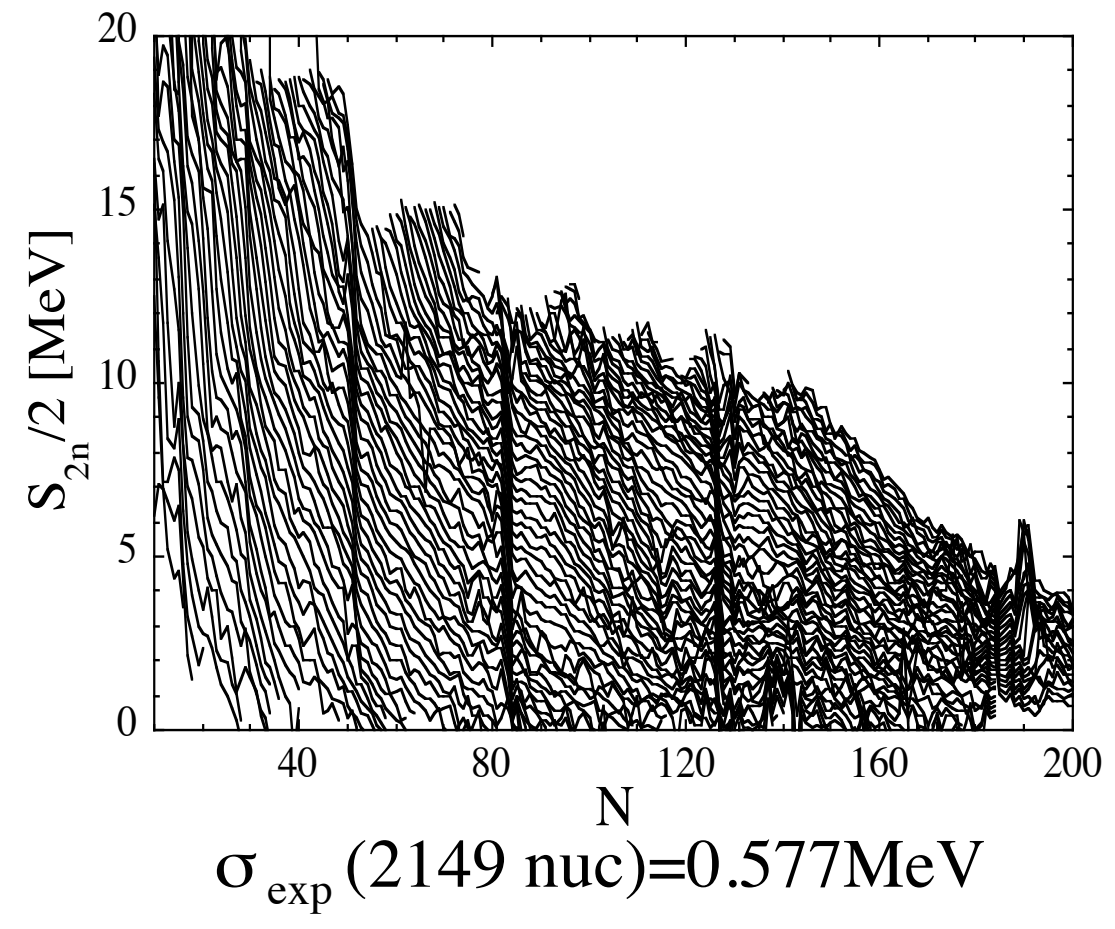

HFB-21 after noise-filtering

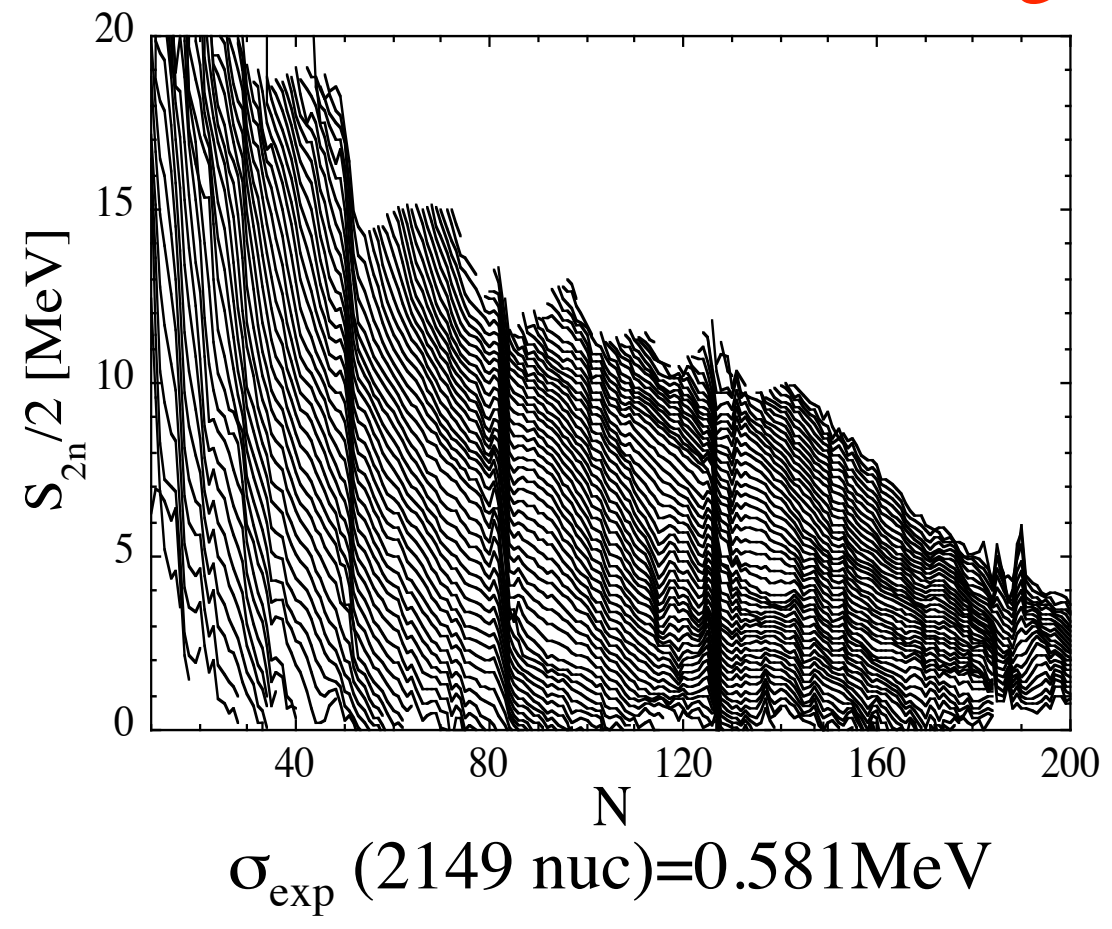

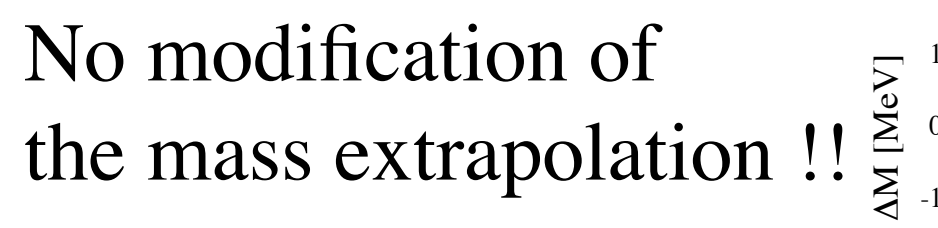

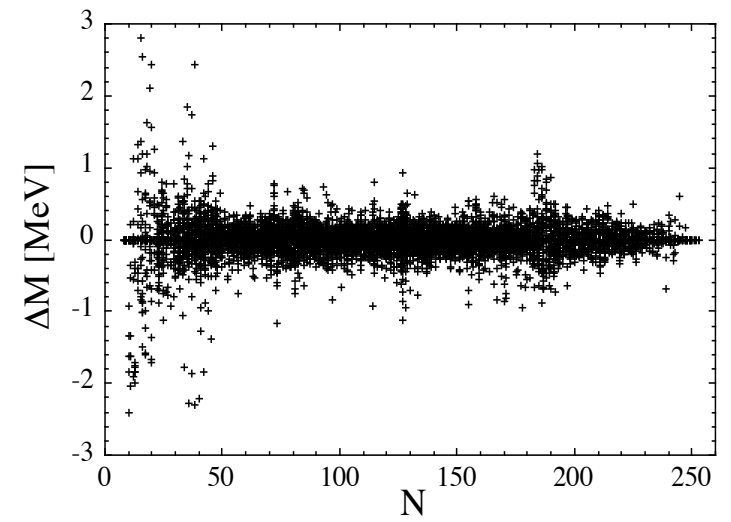




\section{Shell effects}

(of particular interest for r-process applications)

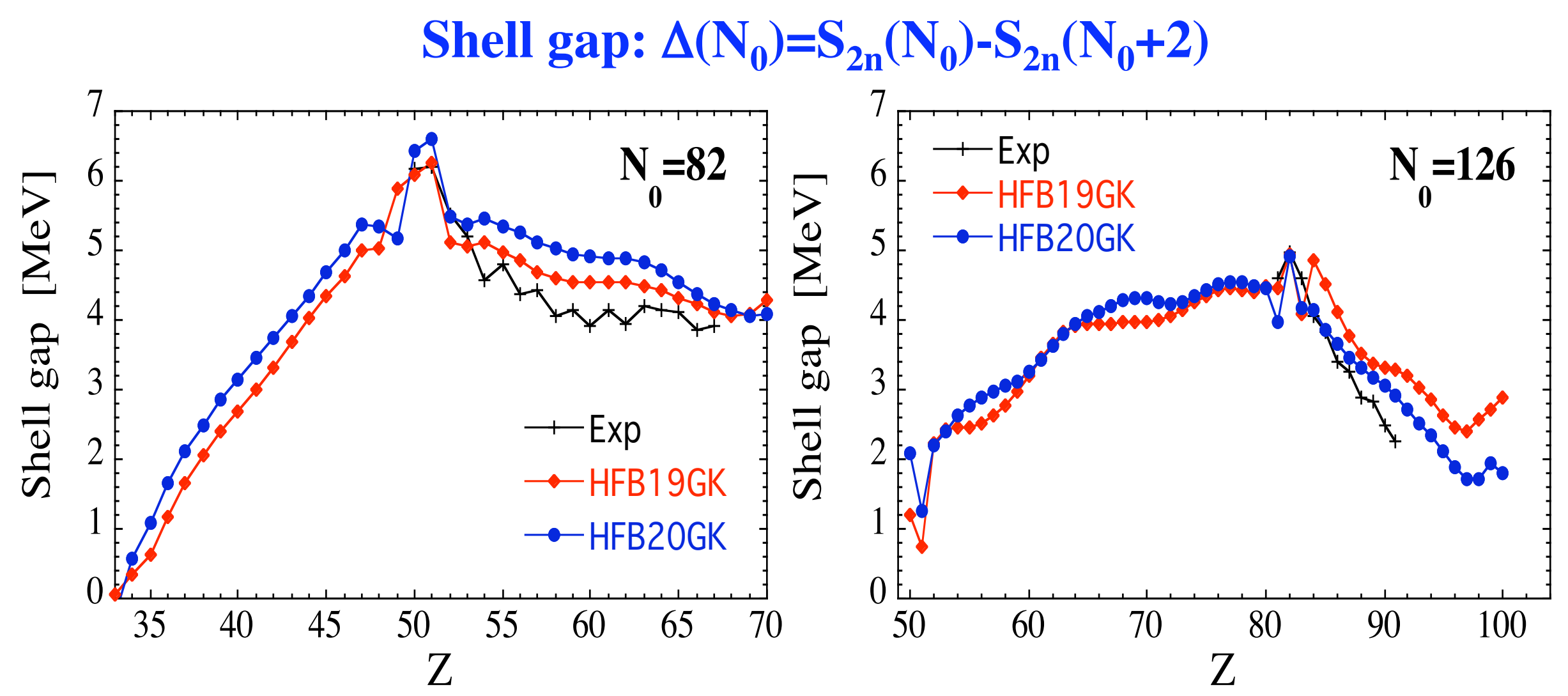


Skyrme-HFB mass models: a first step towards "microscopic" models for practical applications

... but there is obviously still room for many improvements:

- Pairing interaction (contact force, cut-off dependence)

- Improved treatment of odd nuclei

- Phenomenological Wigner correction

- Finite-range forces of Gogny-type

- Correlation effects beyond mean field

- Etc... 
A new generation of mass models

\author{
Gogny-HFB mass table \\ beyond mean field !
}


Beyond the mean field, the total binding energy is estimated from

$$
E_{\text {tot }}=E_{H F B}-E_{Q u a d}
$$

where $\bullet E_{H F B}$ : deformed HFB binding energy obtained with a finite range standard Gogny-type force

$$
\begin{aligned}
V(1,2)= & \sum_{j=1,2} e^{-\frac{\left(\vec{r}_{1}-\vec{r}_{2}\right)^{2}}{\mu_{j}^{2}}}\left(W_{j}+B_{j} P_{\sigma}-H_{j} P_{\tau}-M_{j} P_{\sigma} P_{\tau}\right) \\
& +t_{0}\left(1+x_{0} P_{\sigma}\right) \delta\left(\vec{r}_{1}-\vec{r}_{2}\right)\left[\rho\left(\frac{\vec{r}_{1}+\vec{r}_{2}}{2}\right)\right]^{\alpha} \\
& +i W_{L S} \bar{\nabla}_{12} \delta\left(\vec{r}_{1}-\vec{r}_{2}\right) \times \vec{\nabla}_{12} \cdot\left(\vec{\sigma}_{1}+\vec{\sigma}_{2}\right) .
\end{aligned}
$$

- $E_{\text {Quad }}:$ quadrupolar correction energy determined with the same Gogny force (no "double counting") in the framework of the GCM+GOA model for the five collective quadrupole coordinates, i.e. rotation, quadrupole vibration and coupling between these collective modes (axial and triaxial quadrupole deformations included)

Girod, Berger, Libert, Delaroche 


\section{First Gogny-HFB mass formula (D1M force)}

2149 Masses: $\varepsilon=0.126 \mathrm{MeV} \sigma=0.798 \mathrm{MeV}$ with coherent $E_{Q u a d} \& E_{H F B}$ !

707 Radii: $\varepsilon=-0.008 \mathrm{fm} \sigma=0.031 \mathrm{fm}$ (with Q corrections)
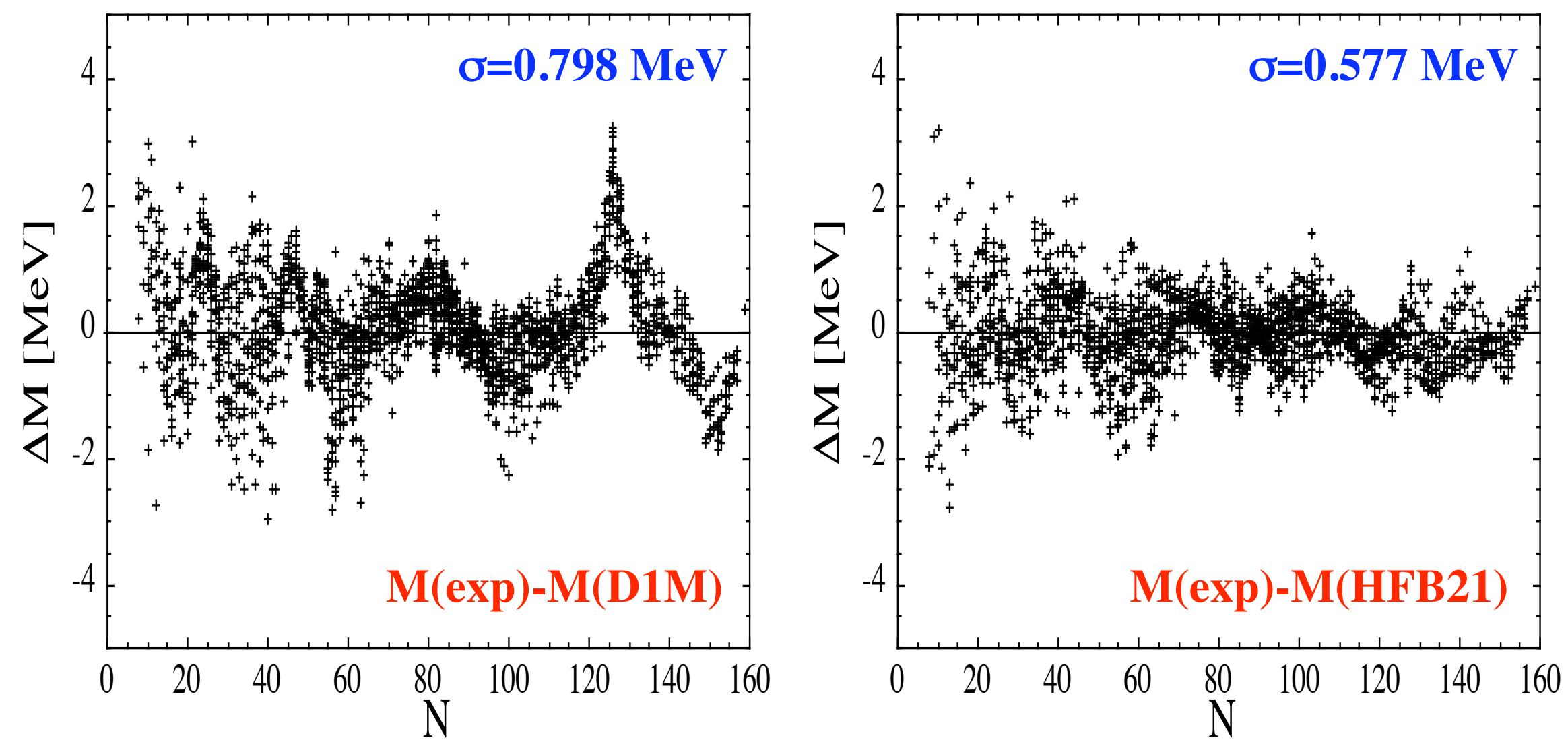

--> It is possible to adjust a Gogny force to reproduce all exp masses accurately 


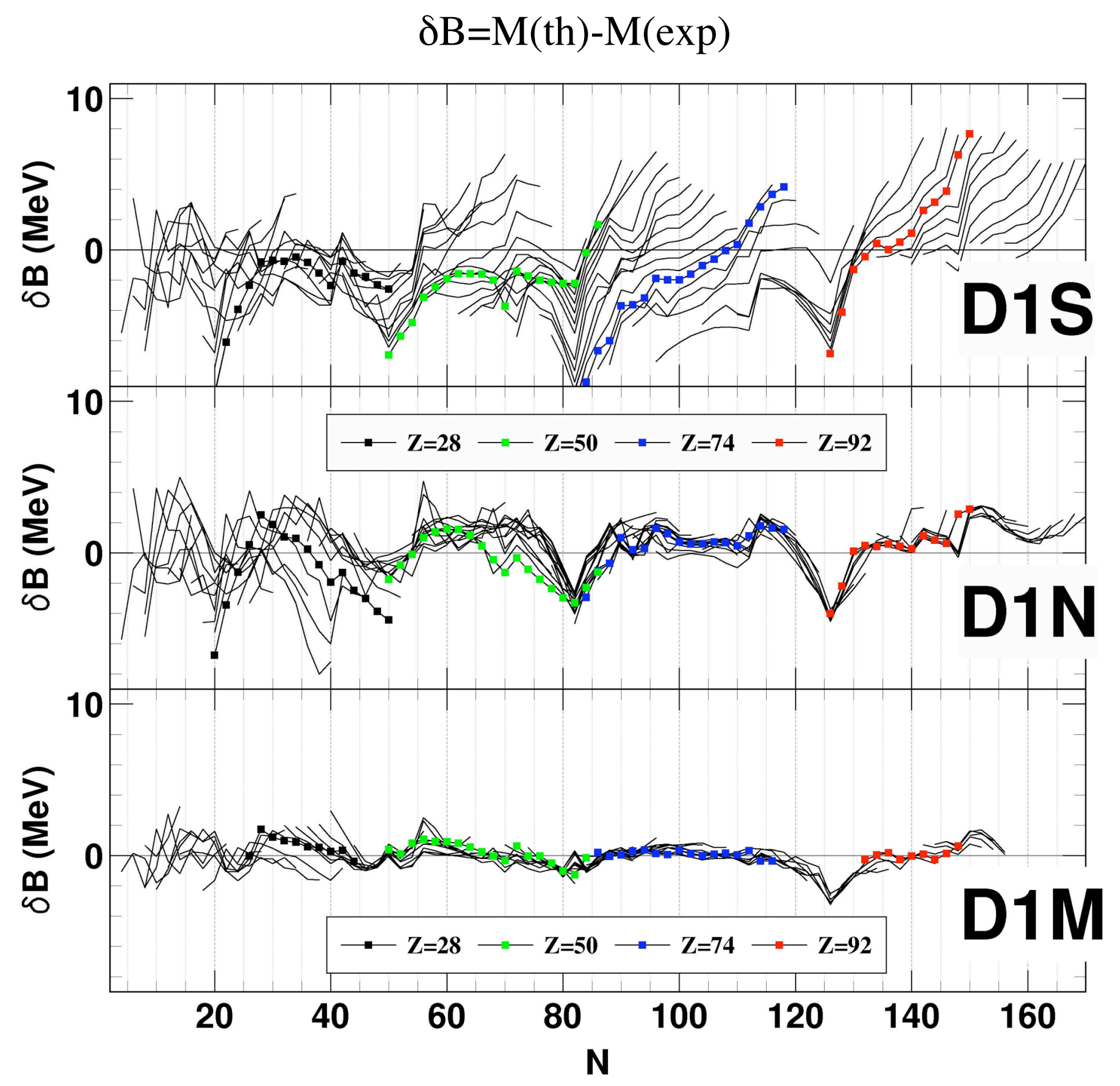


Comparison between Skyrme-HFB, Gogny-HFB and FRDM masses
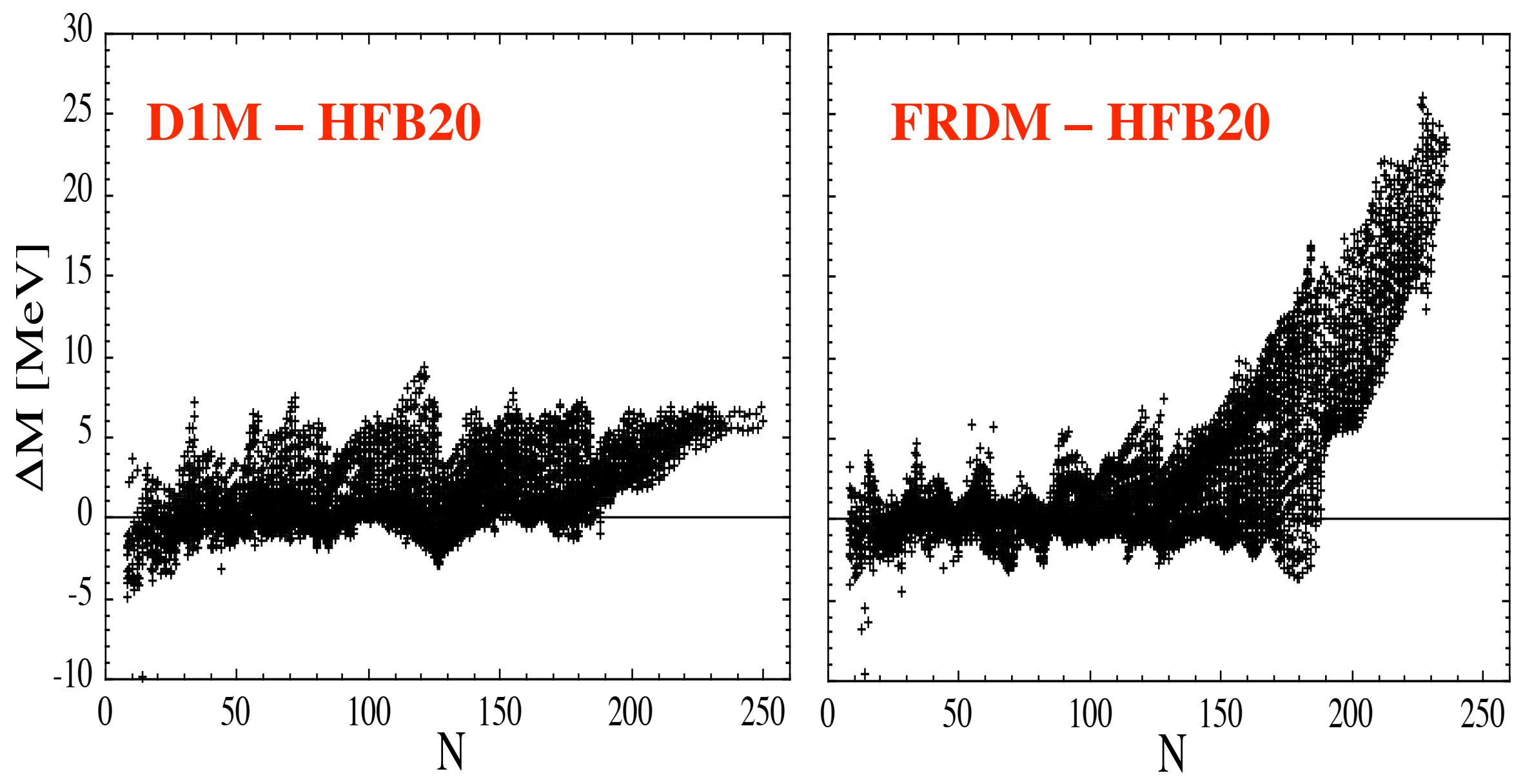
Impact of nuclear masses on the $(\mathrm{n}, \gamma)$ reaction rate at $T=10^{9} \mathrm{~K}$ $(\sim$ cross section around $100 \mathrm{keV}$ - Calculation within the HF reaction model)

Sn isotopes

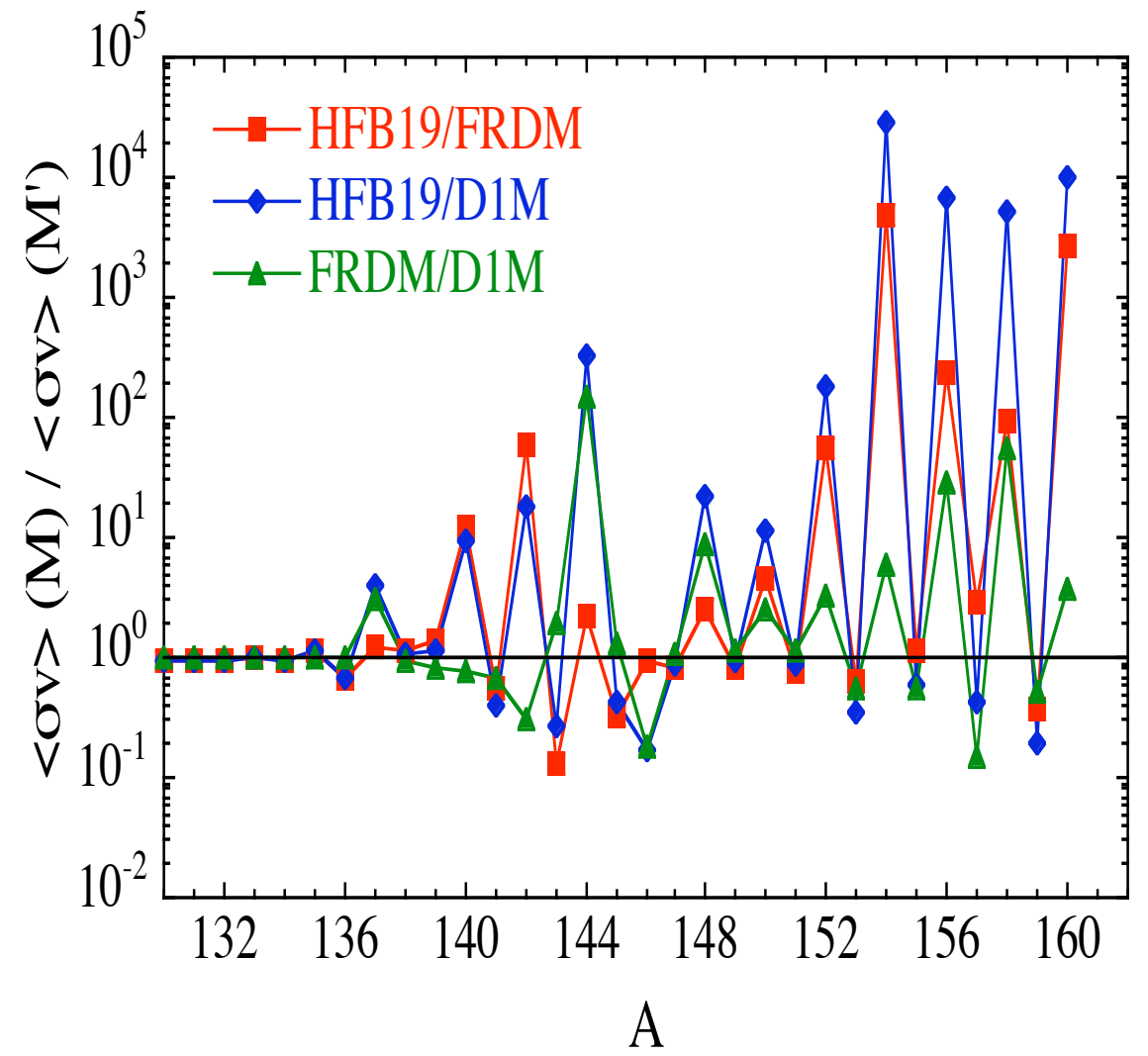

$\mathrm{Pb}$ isotopes

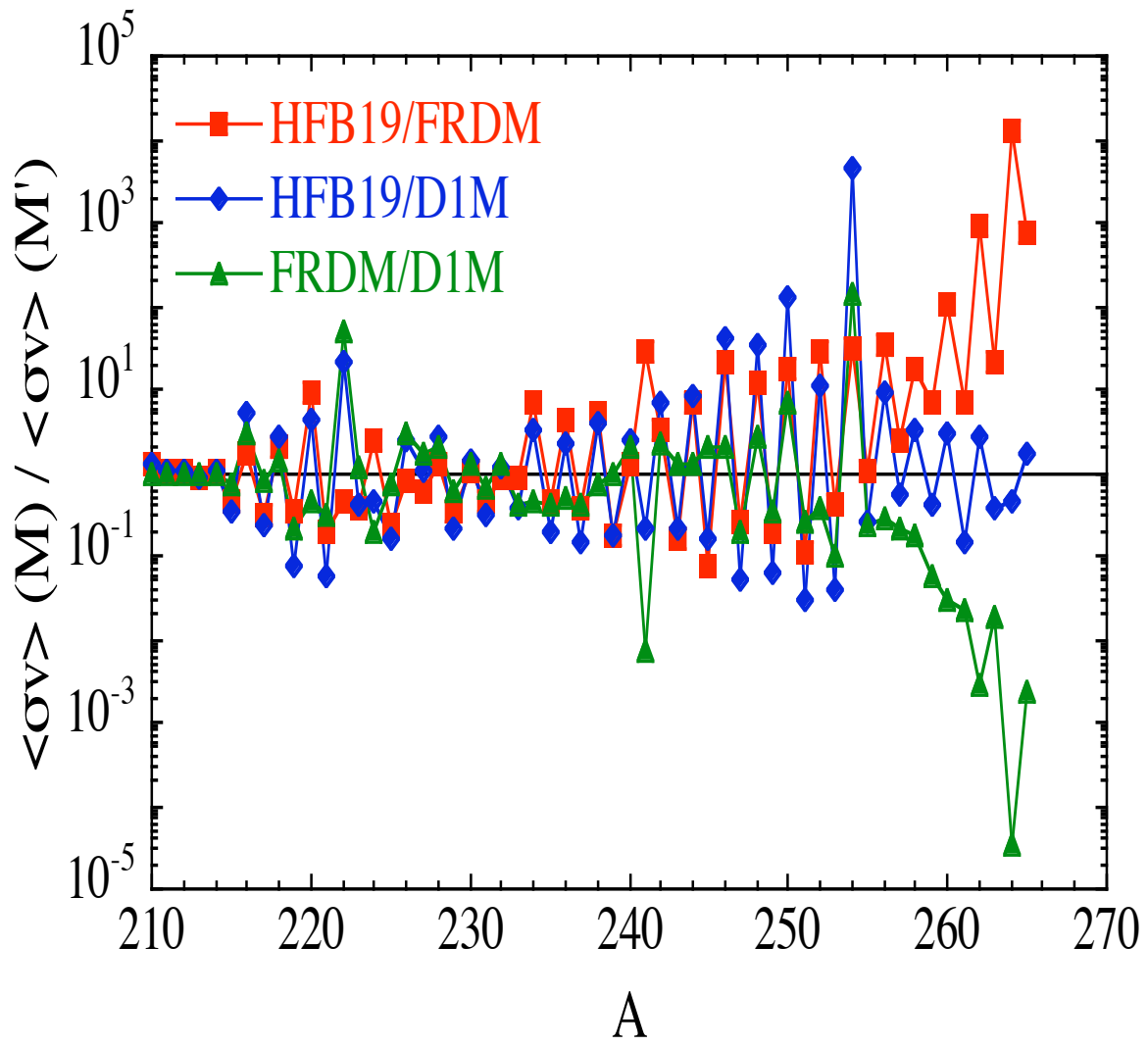


$\mathrm{r}$-process in supernova $v$-driven wind

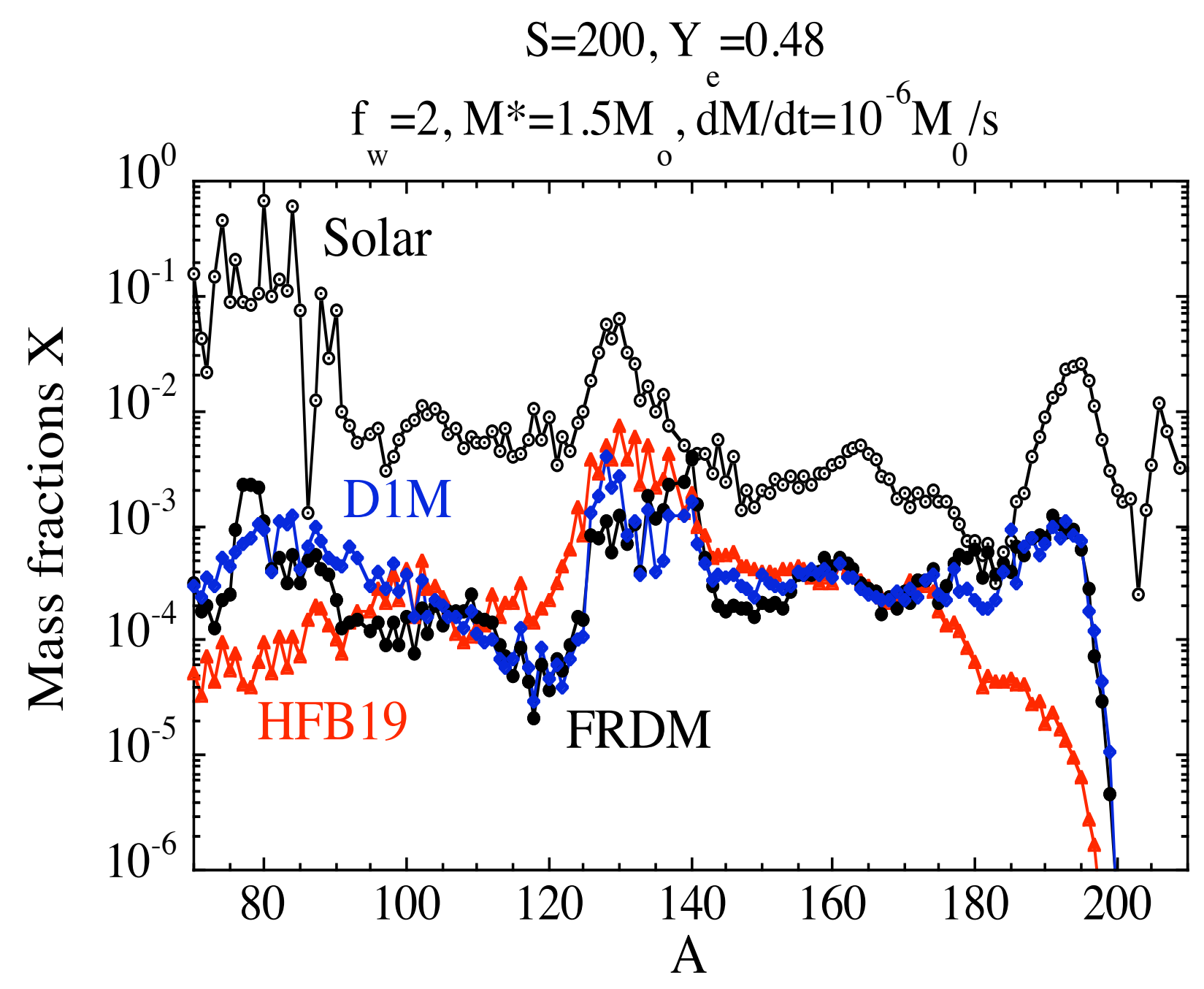




\section{Decompression of Neutron Star matter}

\section{different nuclear ingredients in the nuclear reaction model}
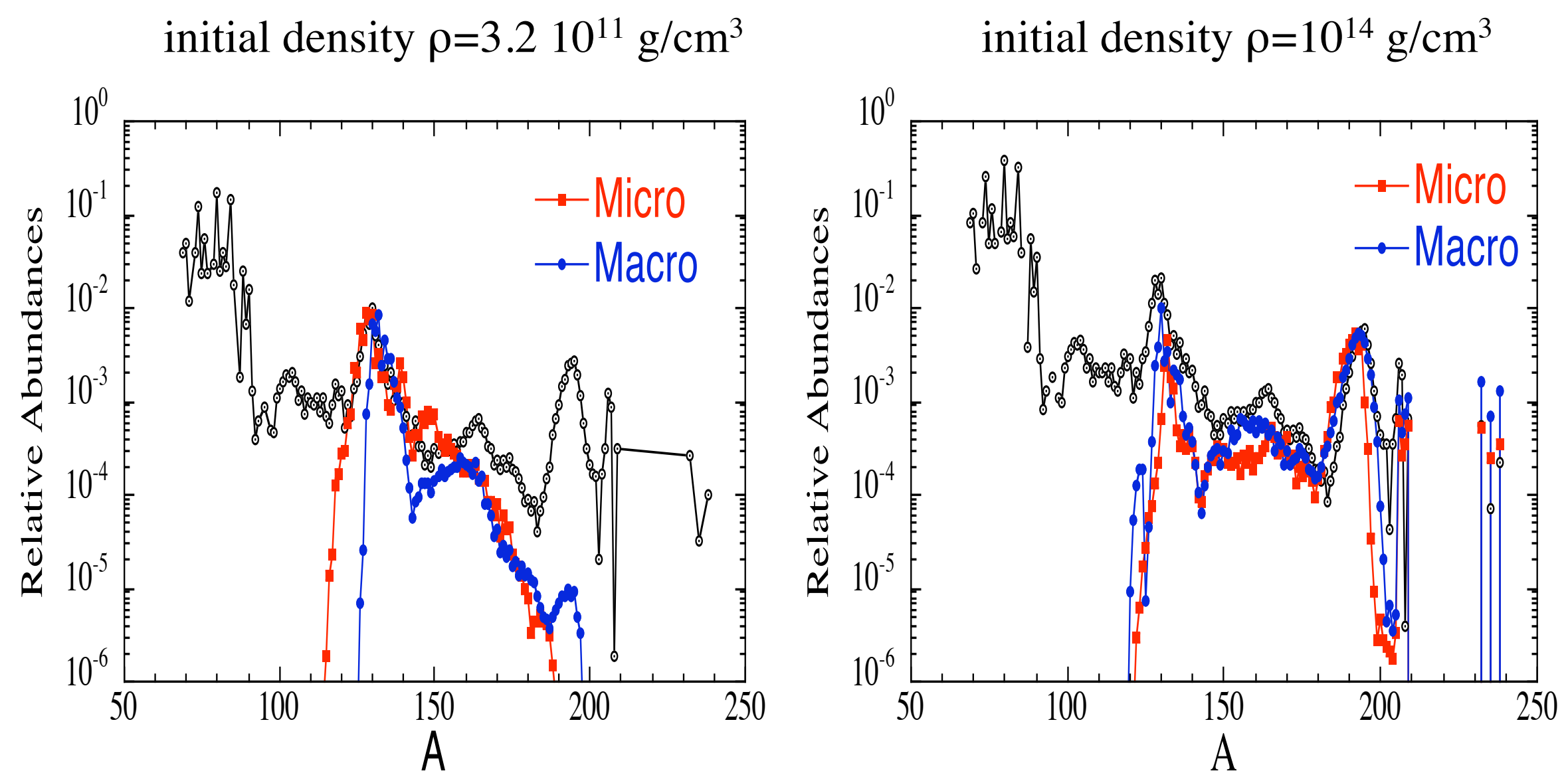

(same $\beta$-decay \& fission rates) 


\section{$\gamma$-ray strength function}




\section{$\gamma$-ray strength function}

Global models available for $\gamma$-ray strength functions:

- Classical Approaches

\section{$\underline{\text { Reliability Accuracy }}$}

Lorentzian model \& Liquid Drop vibration

Lorentzian model with E-dependent width

- Semi-Classical Approaches

Lorentzian model with E- \& T-dependent width

(GLO, EGLO, MLO, GFL, Therm. Pole App., Hybrid, ...)

Semi-Microscopic Model

HFB+QRPA model

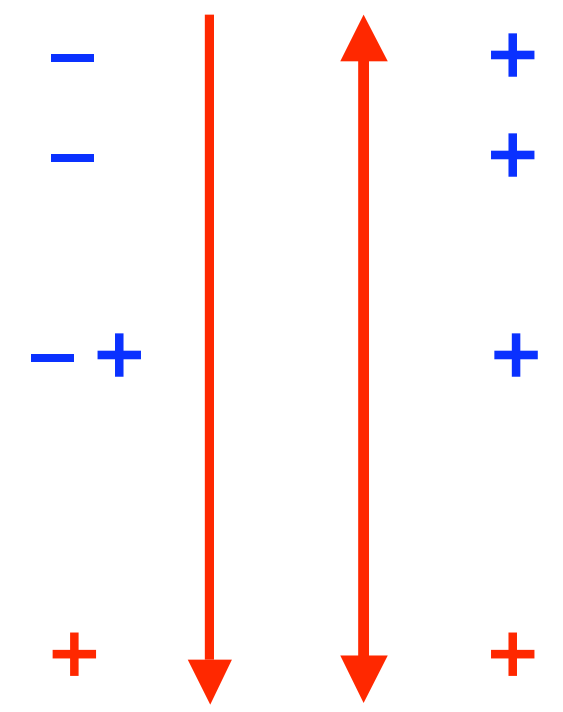

Experimental constraints

- 84 photoabsorption data,

- 50 low-energy strengths from resolved resonances or thermal capture measur.

- $(\gamma, \mathrm{n}),\left(\gamma, \gamma^{\prime}\right)$ experiments

- $\left({ }^{3} \mathrm{He}, \alpha \gamma\right)$ experiments (NLD-model dependent) 


\section{The Lorentzian approach to the $\gamma$-strength function}

- Standard Lorentzian

- Lorentzian with E-dependent width (e.g McCullagh et al. 1981) $\quad \Gamma=\Gamma_{0}\left(\frac{E}{E_{0}}\right)^{1 / 2}$

- Generalized Lorentzian with T- and E-dep. width (e.g Kopecky \& Uhl 1990)

The E- and T-dependent width is essentially derived from the theory of Fermi liquids (e.g Kadmenski et al. 1982) and also suggested by exp. ARC data

$$
\Gamma=\frac{\Gamma_{0}}{E_{0}^{2}}\left(E^{2}+4 \pi^{2} T^{2}\right)
$$

At the basis of the GLO, EGLO, MLO, GFL, Hybrid, ... modele ${ }^{-1}$

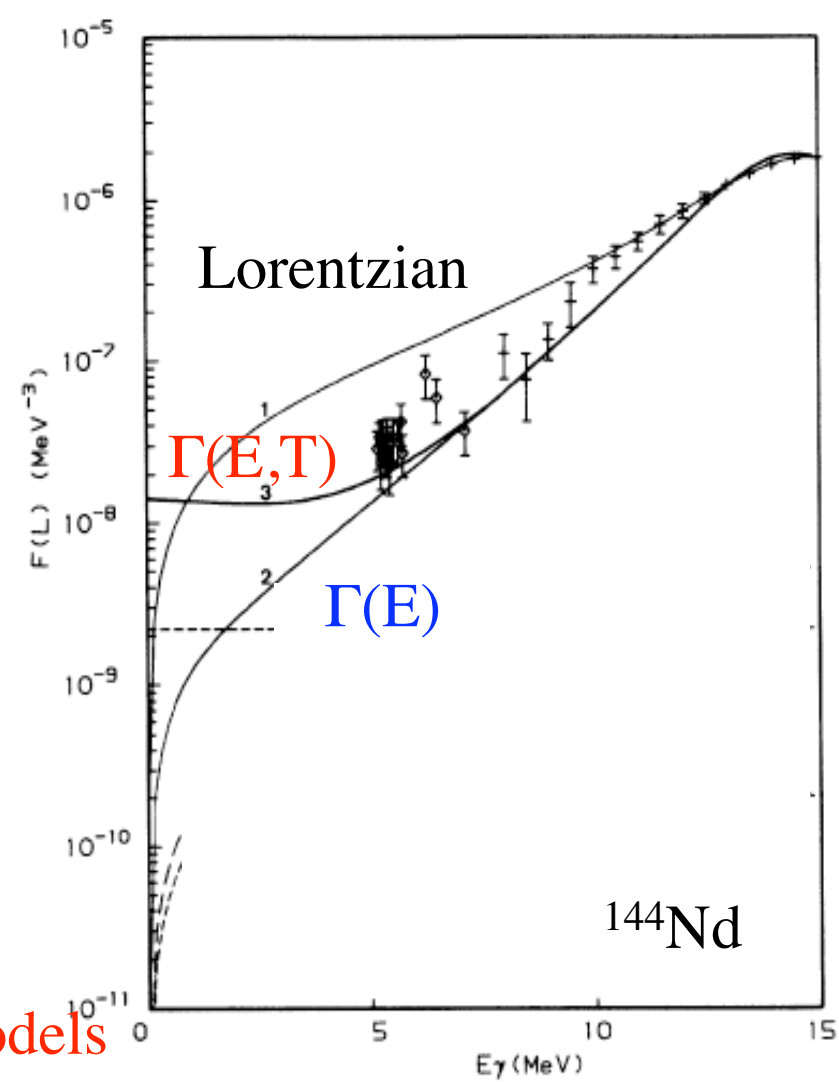

But not many exp. data at low energy to confirm this behaviour Kopecky \& Uhl (1990) 


\section{QRPA $\gamma$-ray strength function}

1. QRPA estimate of the E1-strength distribution based on the Skyrme force

- HFBCS with SLy4 force

- $\quad \mathrm{HFB}$ with BSk7 force

$\longrightarrow$ Comparison of exp. GDR and QRPA centroid energies

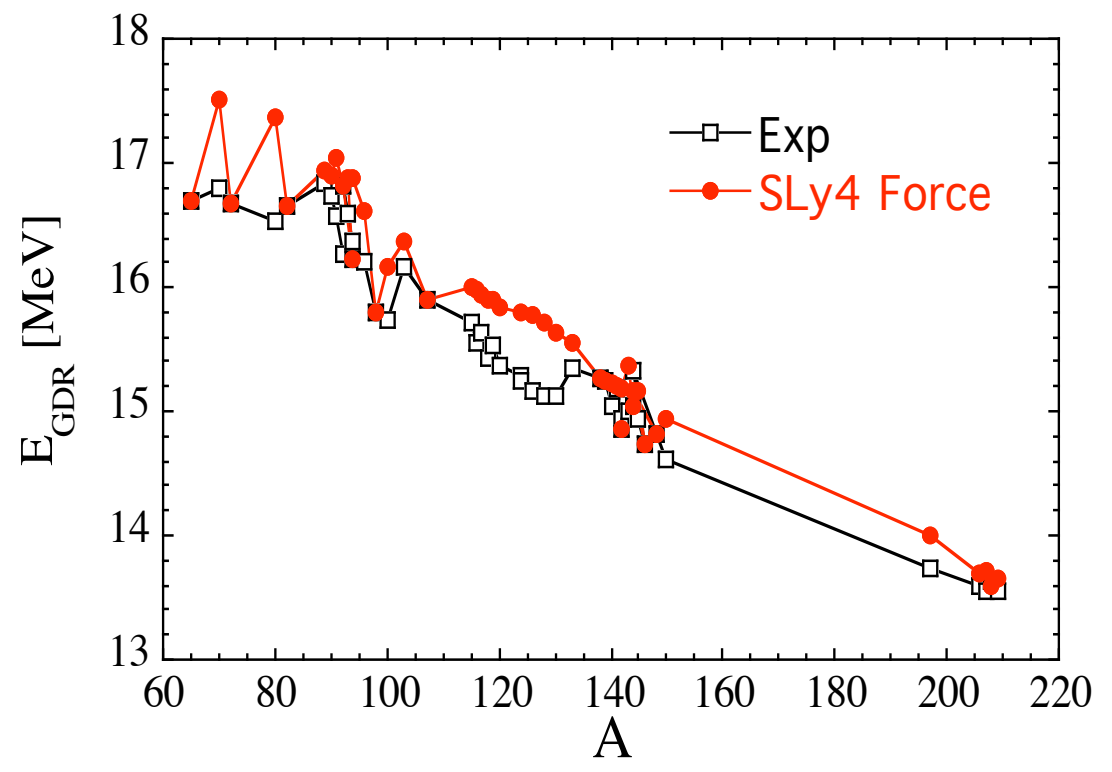

2. Empirical damping of the collective motions: broadening of the E1-strength distribution based on a folding procedure to reproduce photoabsorption and average resonance capture (ARC) data

3. Empirical corrections for deformation effects: splitting of each QRPA strength into two peaks

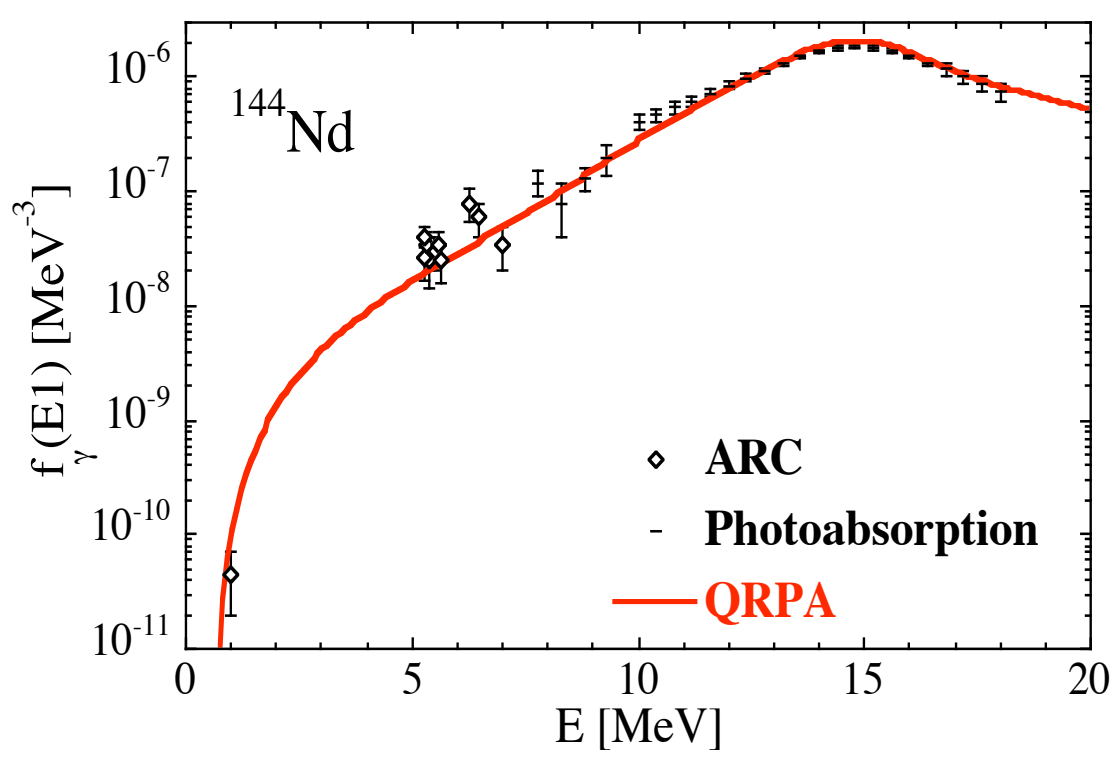




\section{HFB+QRPA prediction of photoabsorption cross section}

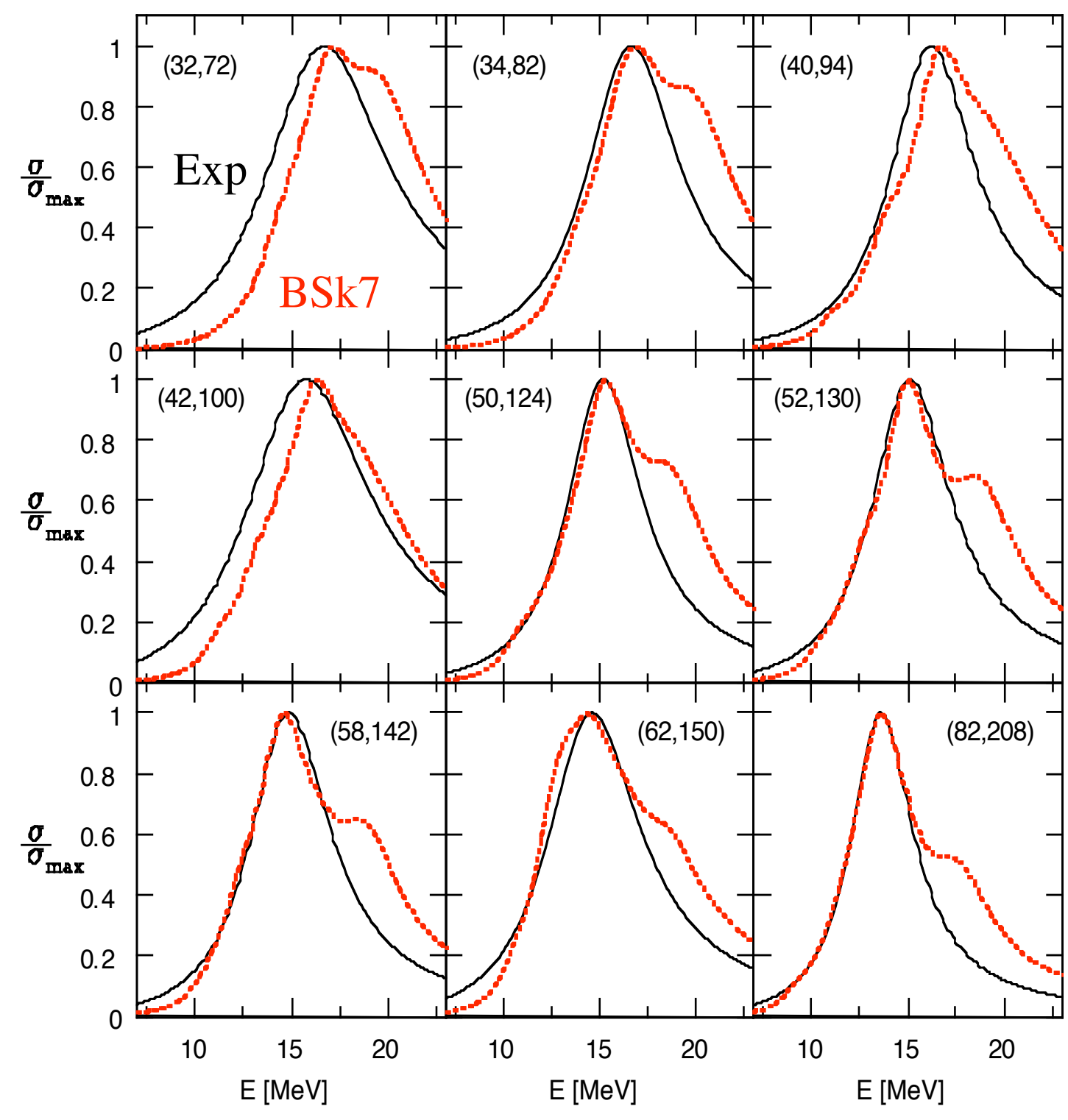




\section{Comparison with experimental data}

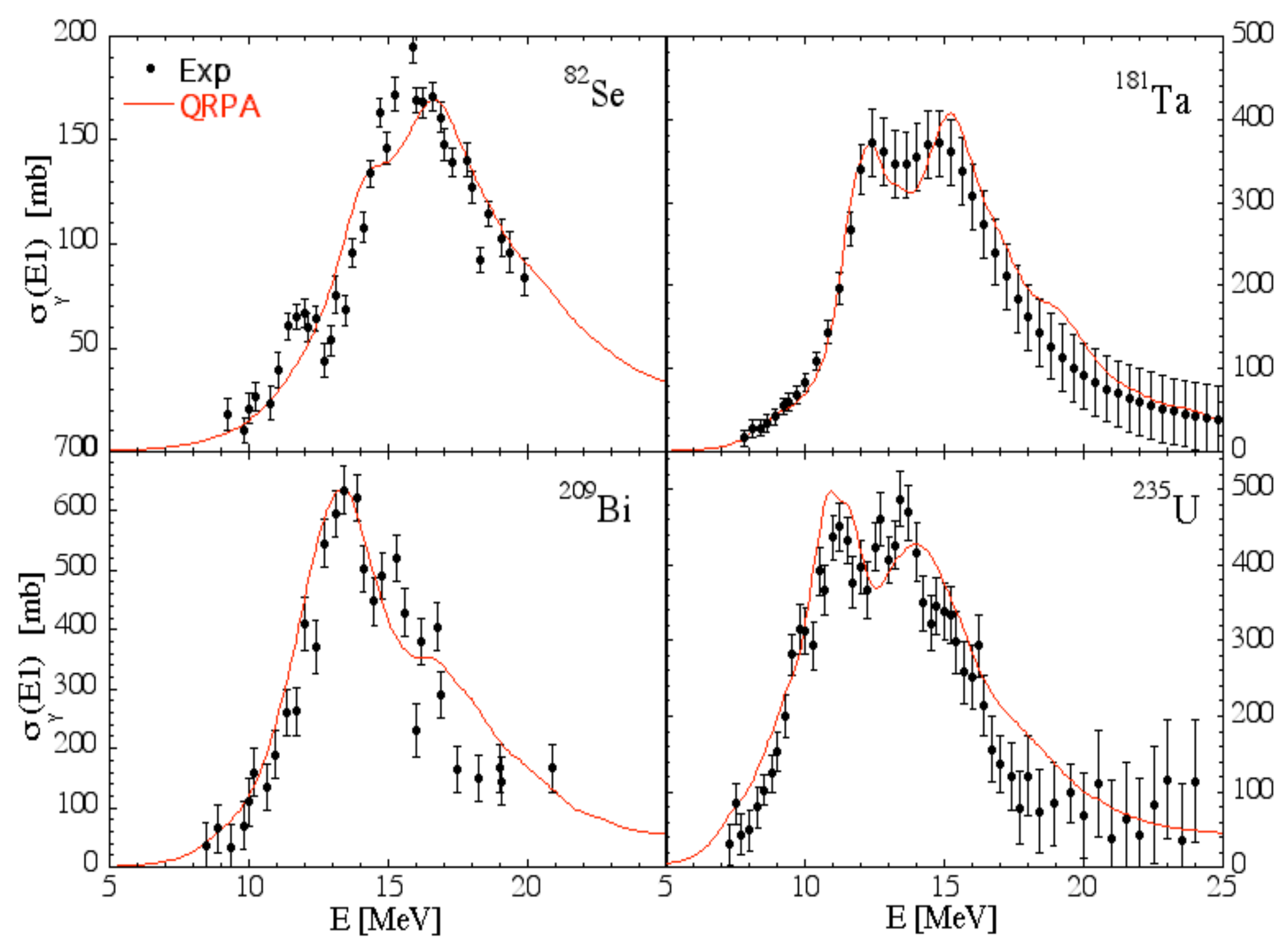




\section{Prediction of E1 strength function}

\section{Low-energy tail of the E1 strength function}

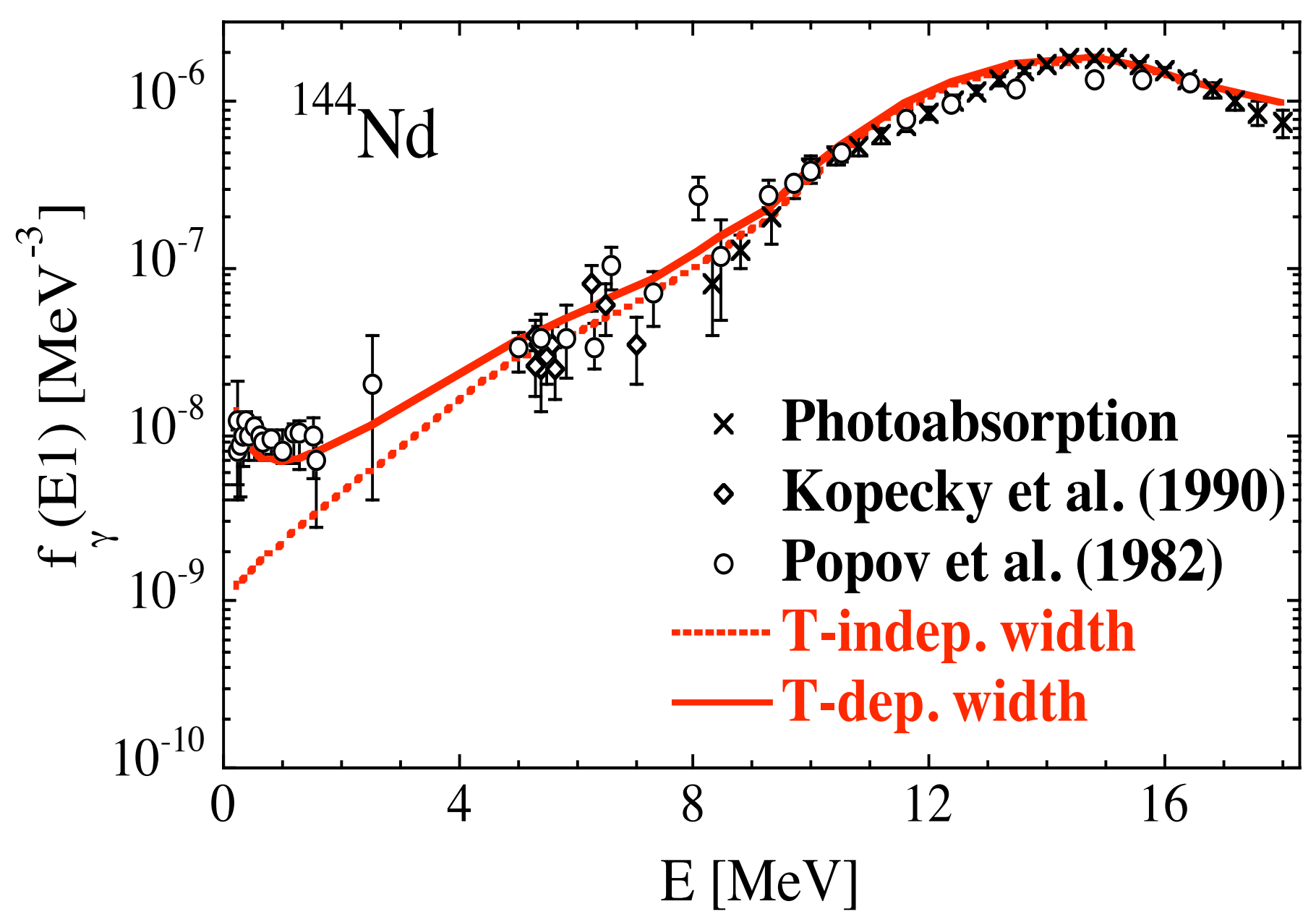




\section{Prediction of E1 strength function}

Resolved-resonance and thermal capture measurements, RIPL2 (2004)

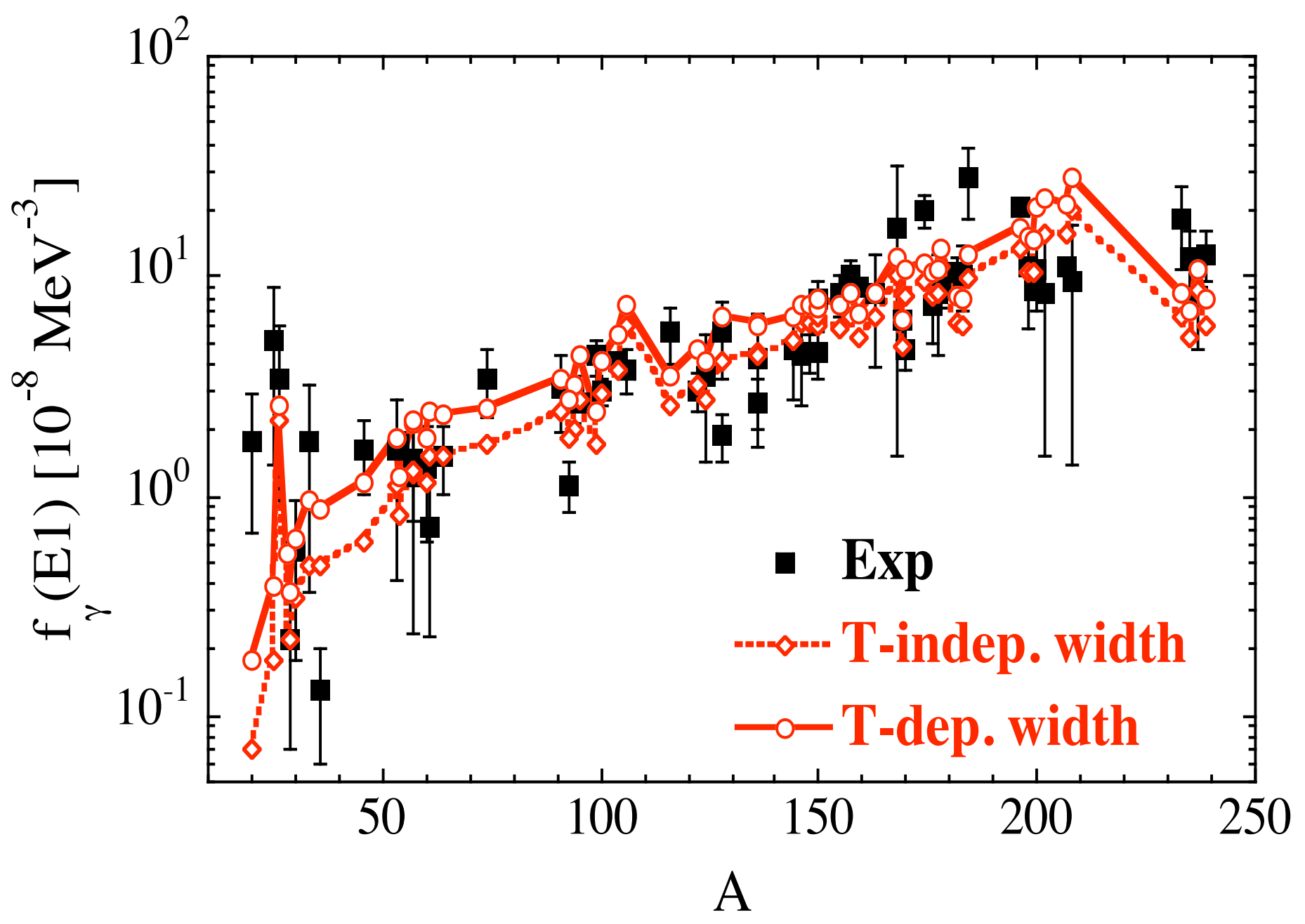


${ }^{90-96} \operatorname{Zr}(\mathrm{n}, \gamma) \&(\gamma, \mathrm{n})$

cross sections

$\gamma$-ray strength constrained on $(\gamma, n)$ xs

E1 HFB+QRPA + M1 GR versus

LORENTZIAN

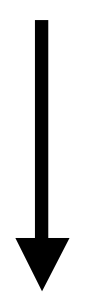

Prediction for short-lived ${ }^{95} \mathrm{Zr}$

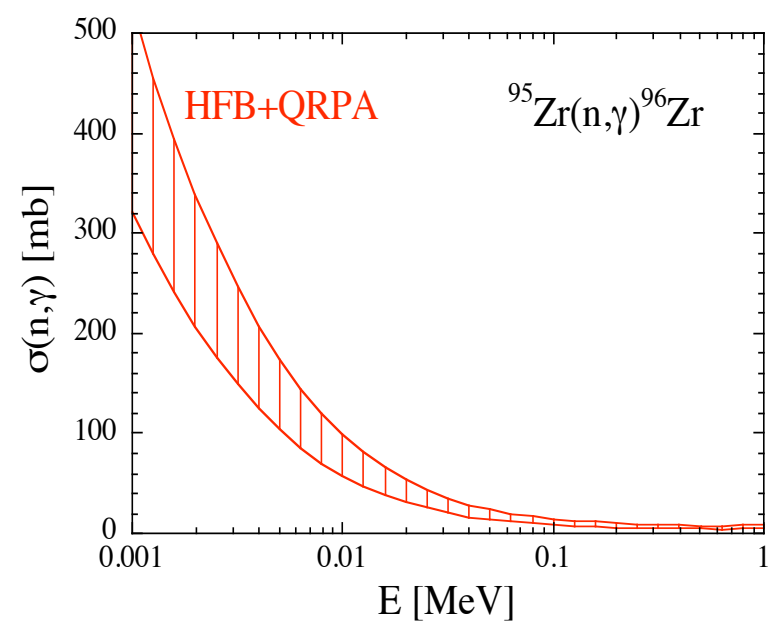

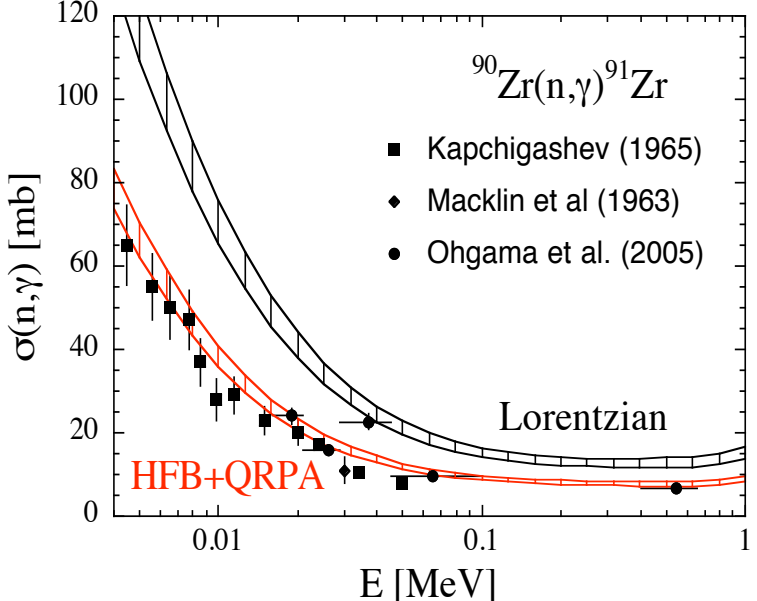
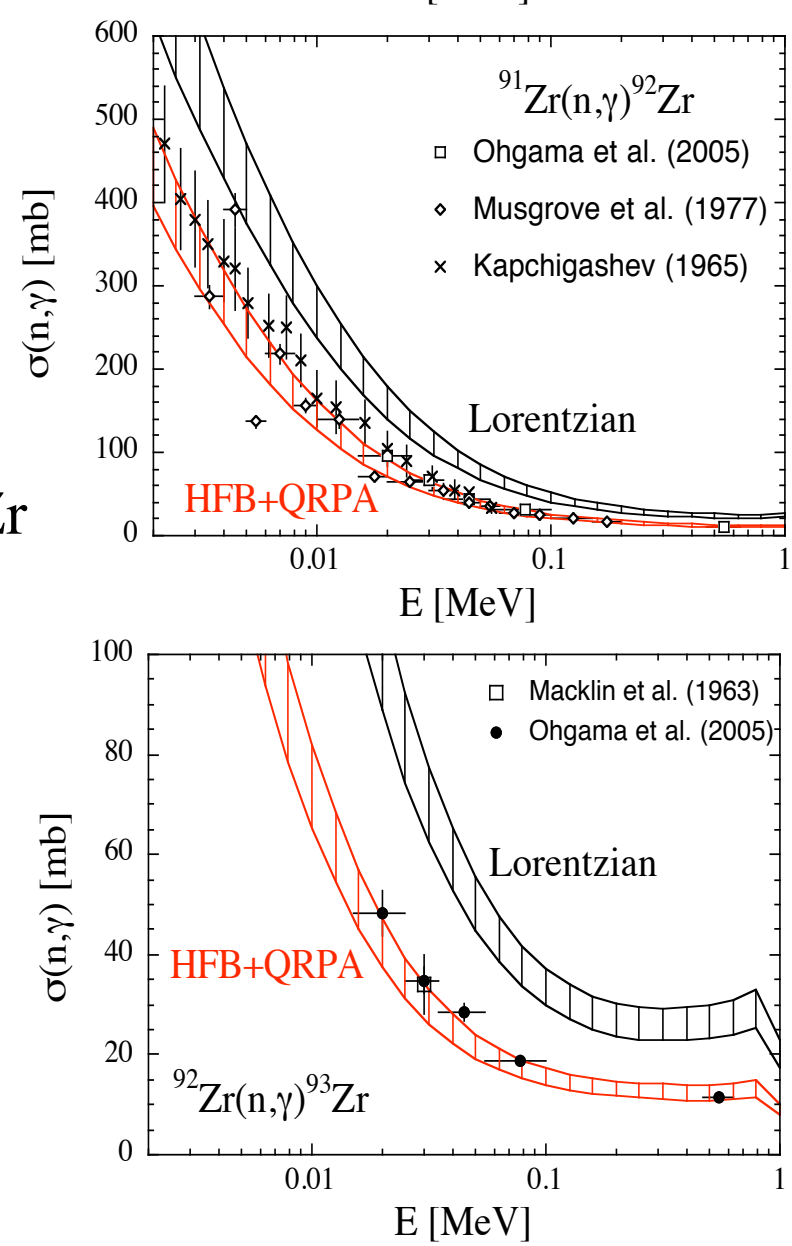
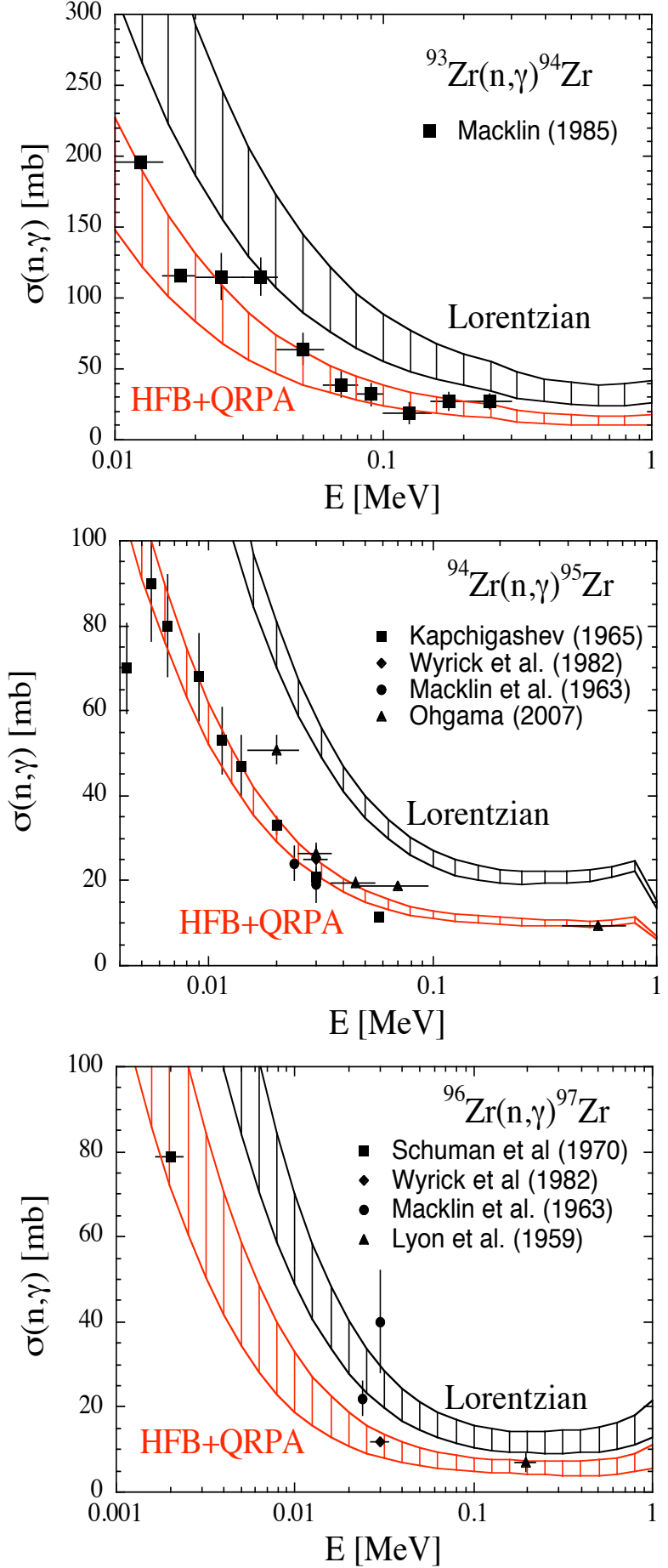


\section{Far away from stability}

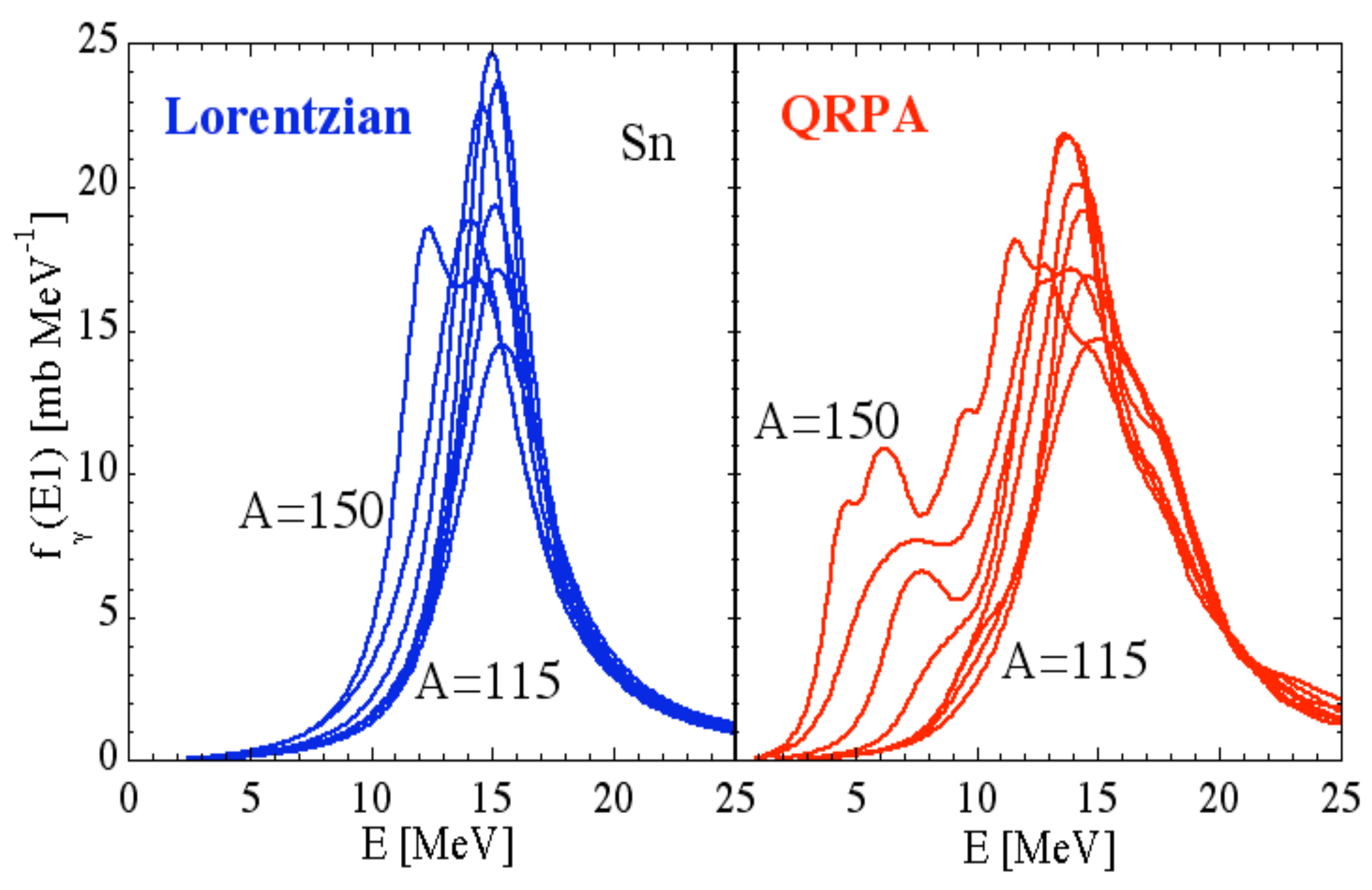


E1 strength models

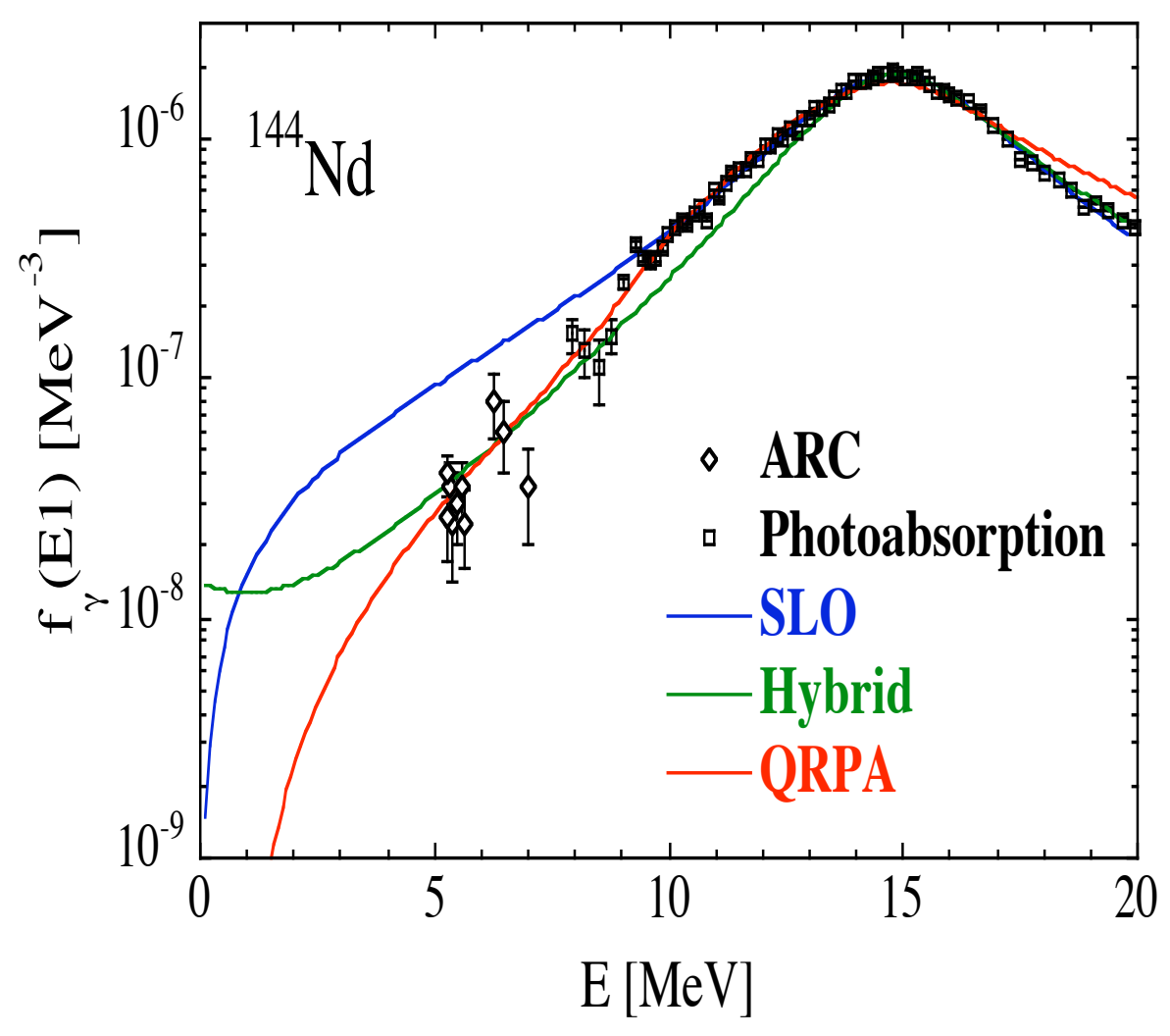

Radiative n-capture rate at $\mathrm{T}=10^{9} \mathrm{~K}$

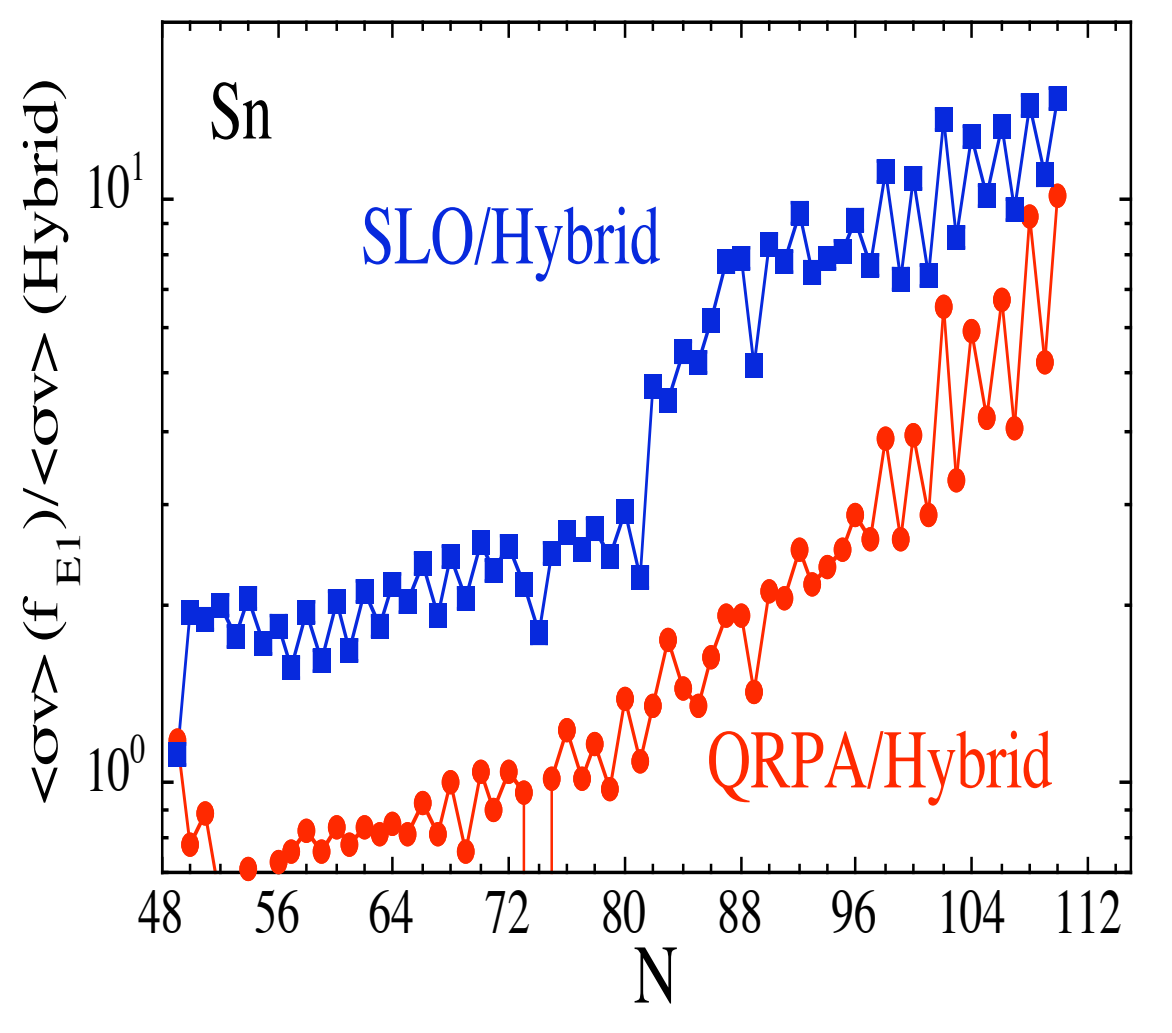

$\sim$ factor of 2 in the n-deficient region

$\sim$ factor of 10 in the $n$-rich region 


\section{Impact on the radiative neutron capture by exotic nuclei}

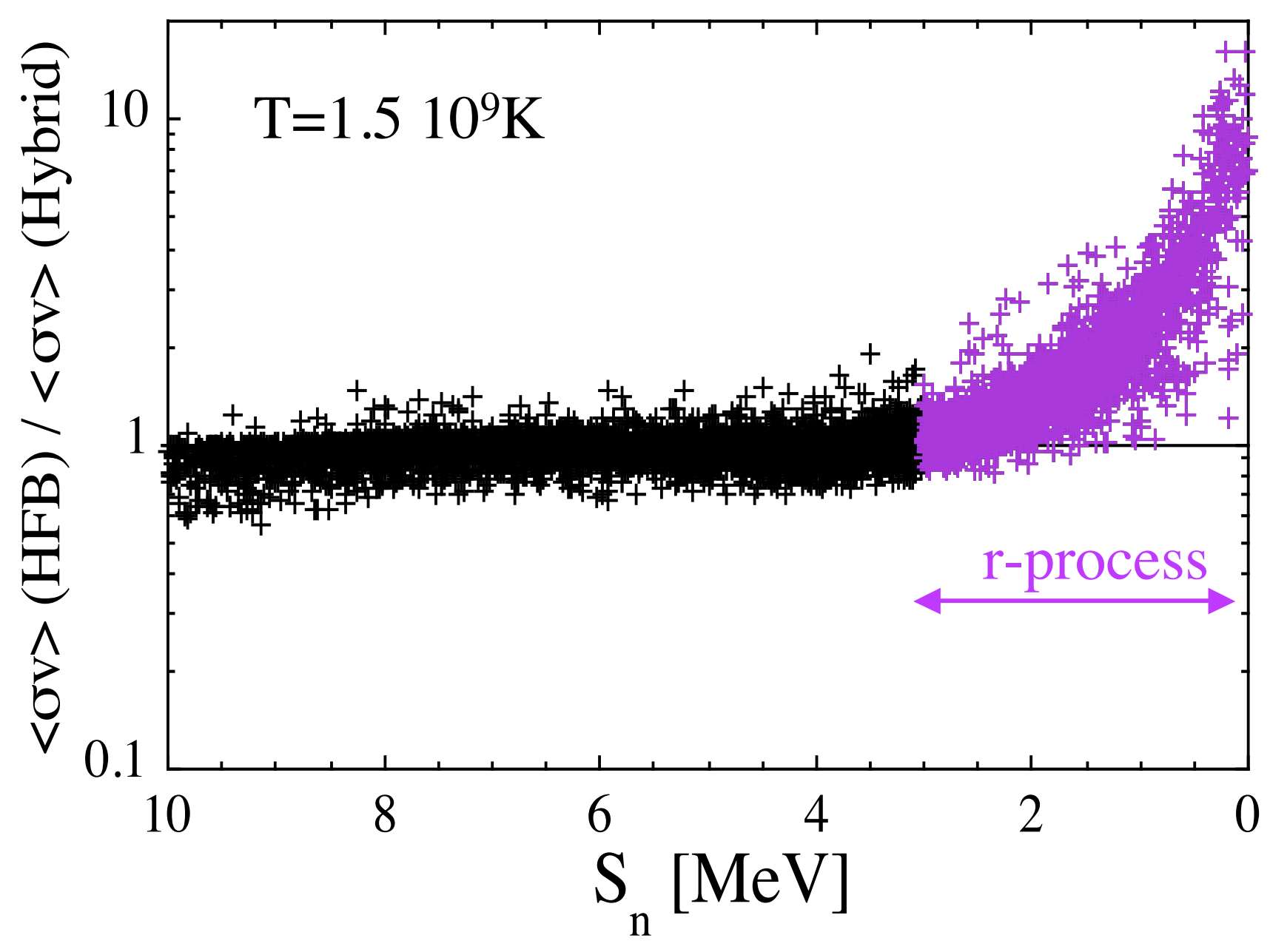


Self-consistent microscopic theories taking into account the single-particle continuum and phonon coupling ( $1 \mathrm{p} 1 \mathrm{~h} \times$ phonon and $2 \mathrm{p} 2 \mathrm{~h} \times$ phonon)

DTBA: Discrete Time Blocking Approximation

Avdeyenkov et al.

- Natural spreading of the strength

- Even more strength at low-energy wrt RPA
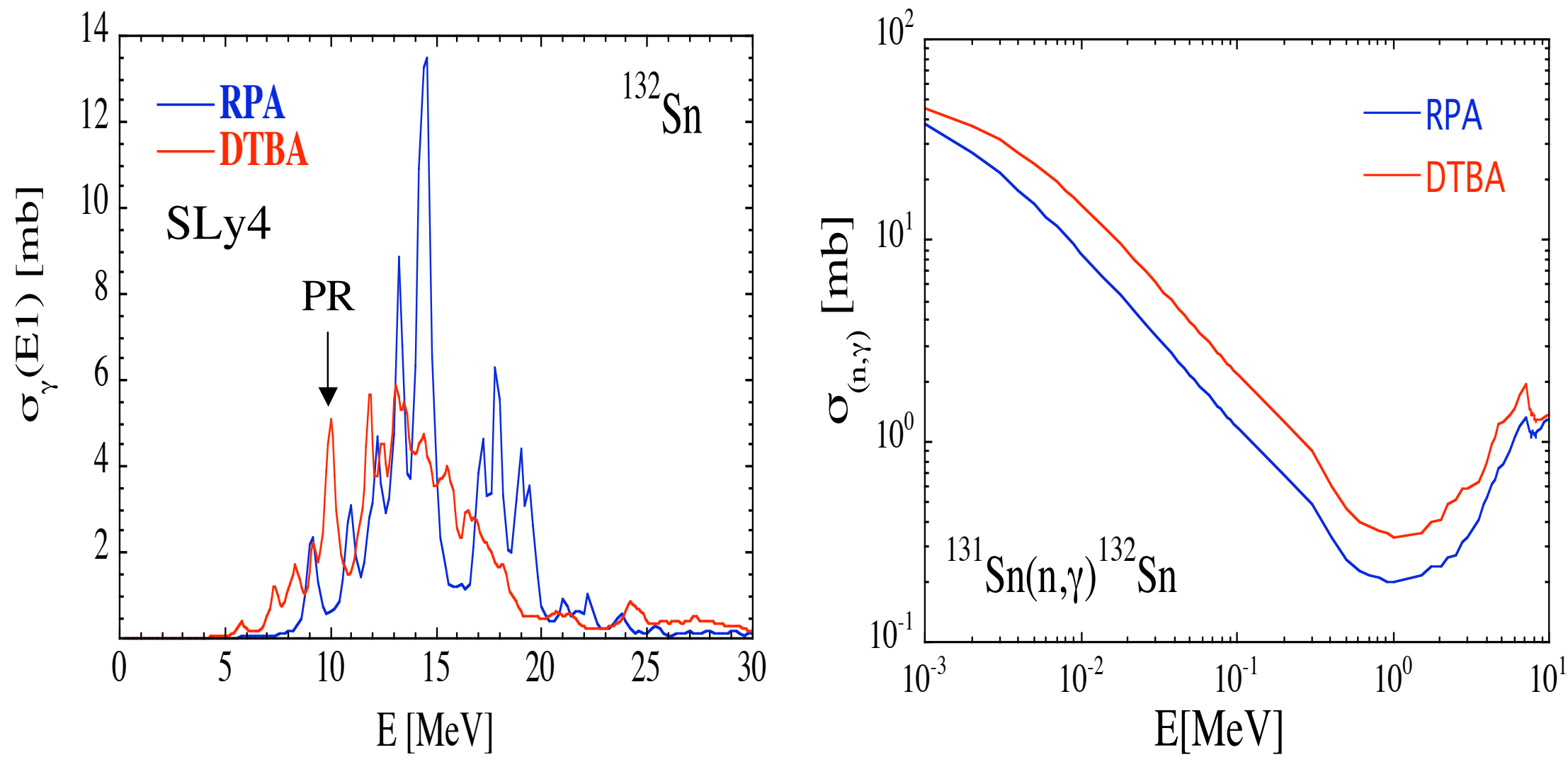

$\begin{array}{lll}\text { Exp: } & E=16.1 \mathrm{MeV} & \Gamma=4.7 \mathrm{MeV} \\ \text { DTBA: } & \mathrm{E}=14.3 \mathrm{MeV} & \Gamma=3.7 \mathrm{MeV}\end{array}$




\section{Far away from stability}
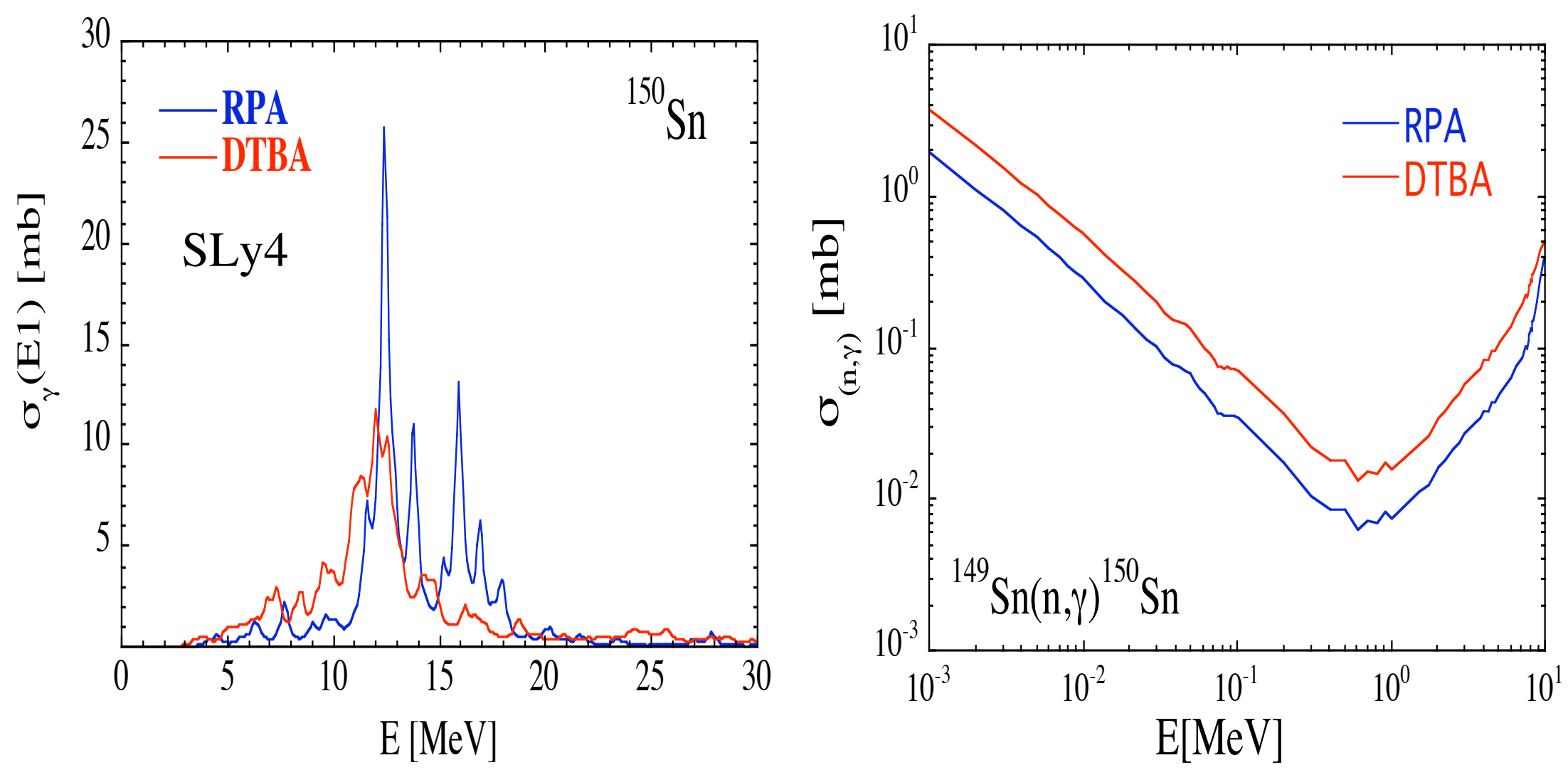

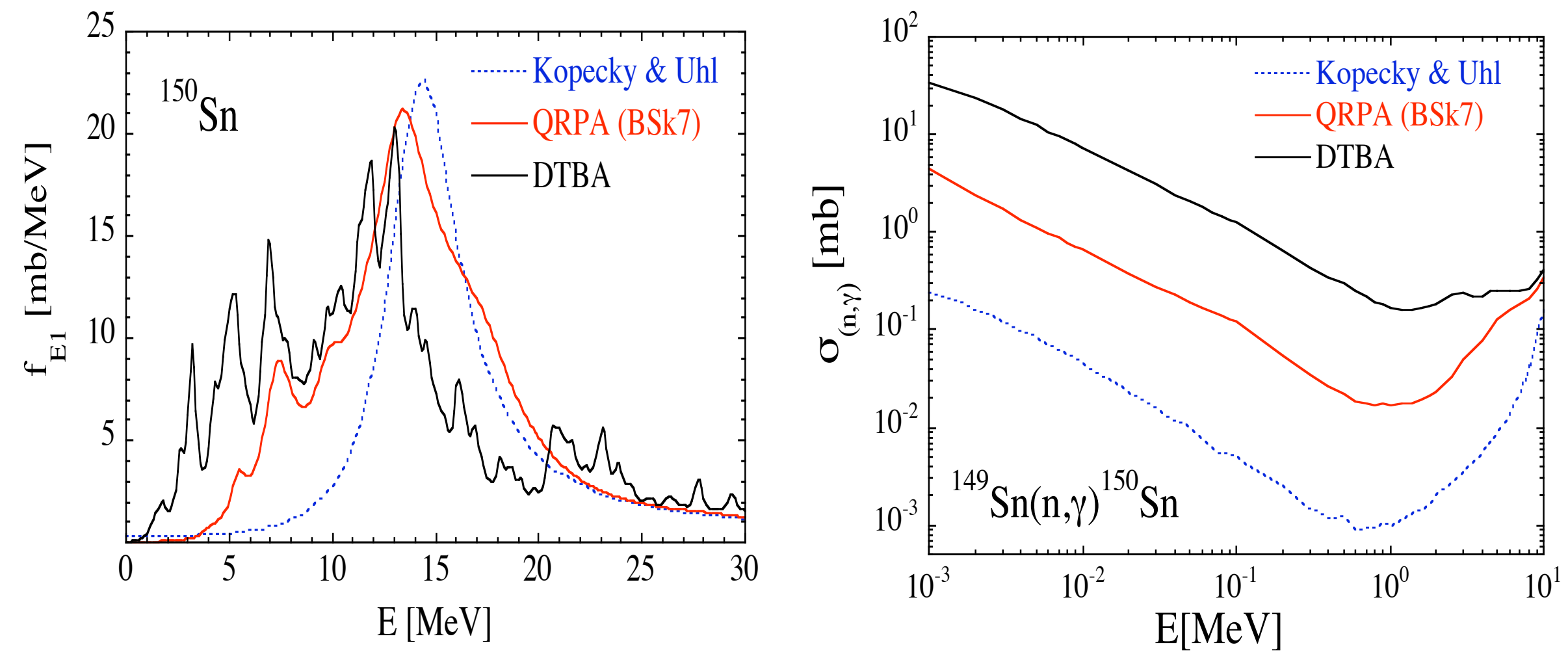


\section{Relativistic QRPA estimates including particle-phonon coupling}

Litvinova et al 2009

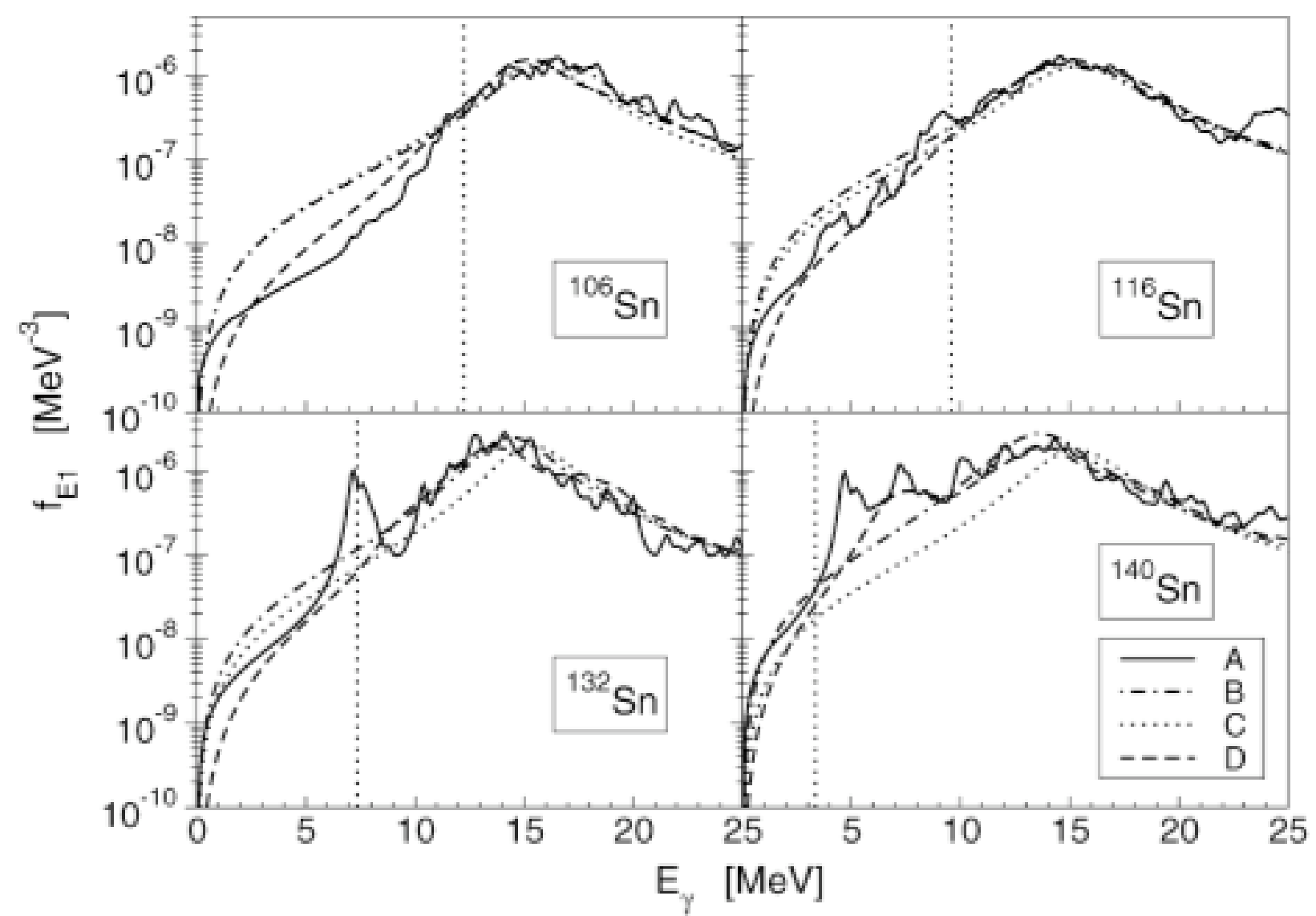

A - RQTBA(Relativistic Quasi-Particle Time Blocking Approximation): Litvinova et al 2009

B - Lorentzian fitted to RQTBA (with E-dependent width)

C - Lorentzian from systematics (with E-dependent width)

D - HFBCS + QRPA (SLy4) 
The low-energy upbend structure observed experimentally in Oslo particle- $\gamma$ coincidence in the $\left({ }^{3} \mathrm{He}, \alpha \gamma\right) \&\left({ }^{3} \mathrm{He},{ }^{3} \mathrm{He}\right.$ ' $\left.\gamma\right)$ reactions

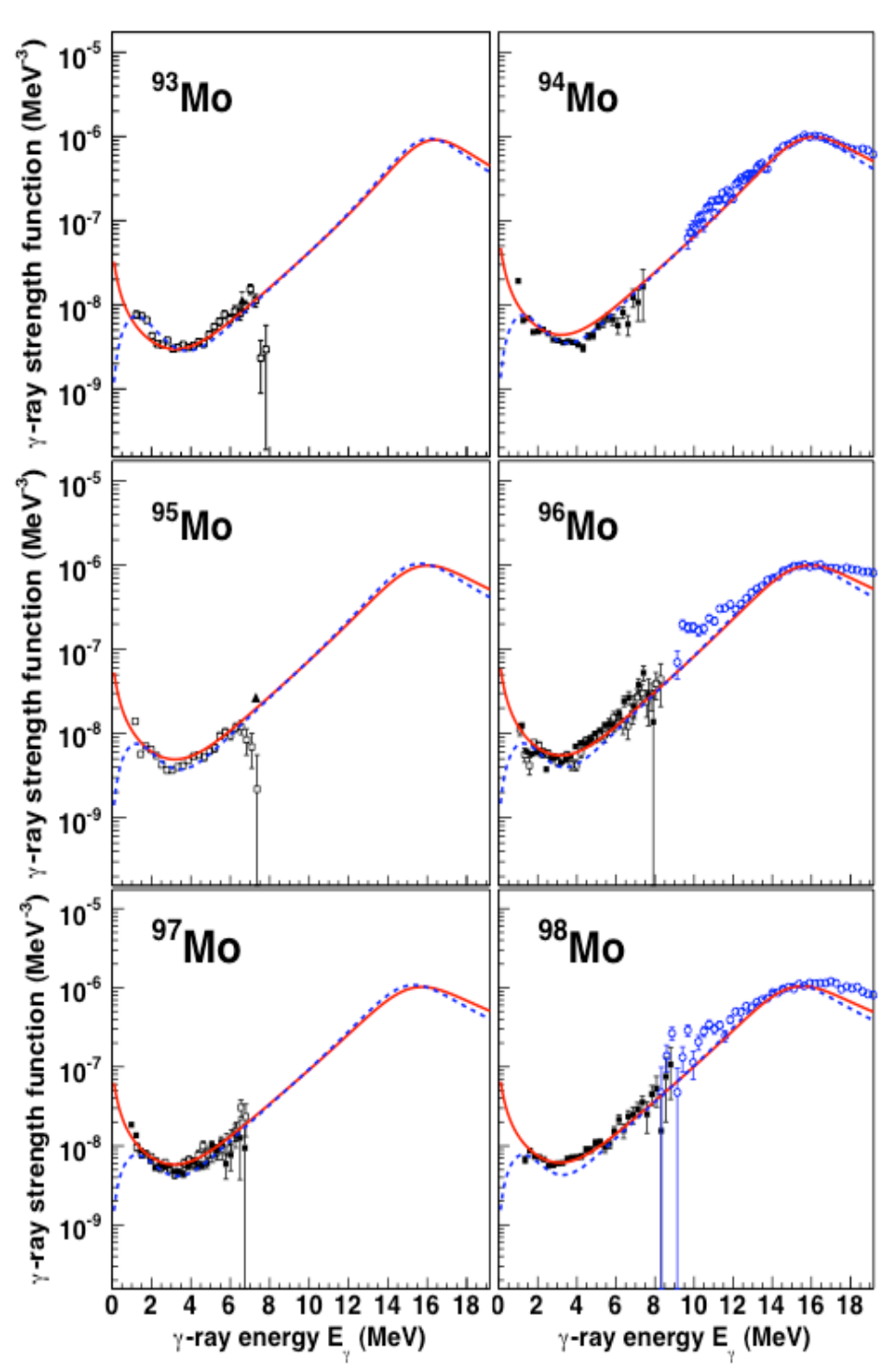

A.-C. Larsen et al. (2009)
Upbend observed for ${ }^{44,45} \mathrm{Sc},{ }^{50,51} \mathrm{~V},{ }^{56,57} \mathrm{Fe}$, ${ }^{93-98} \mathrm{Mo}$, but not for $\mathrm{Sn}, \mathrm{Sm}, \mathrm{Dy}, \mathrm{Er}$ or $\mathrm{Yb}$

Assuming an E1 character, the upbend can be described by a simple phenomenological formula:

Generalized Lorentzian with E-, T-dep. width $f_{E 1}\left(E_{\gamma}\right)=8.68 \times 10^{-8} \frac{\sigma_{0} E_{\gamma} \Gamma\left(E_{\gamma}\right) \Gamma_{0}}{\left.\left(E_{\gamma}^{2}-E_{0}^{2}\right)^{2}+\Gamma_{0} \Gamma\left(E_{\gamma}\right) E_{\gamma}^{2}\right)}$ with $\Gamma\left(E_{\gamma}\right)=\frac{\Gamma_{0}}{E_{0}^{2}}\left[E_{\gamma}^{2}+4 \pi^{2} T_{f}^{2} \frac{E_{0}^{2}}{E_{\gamma}^{2}}\right]$

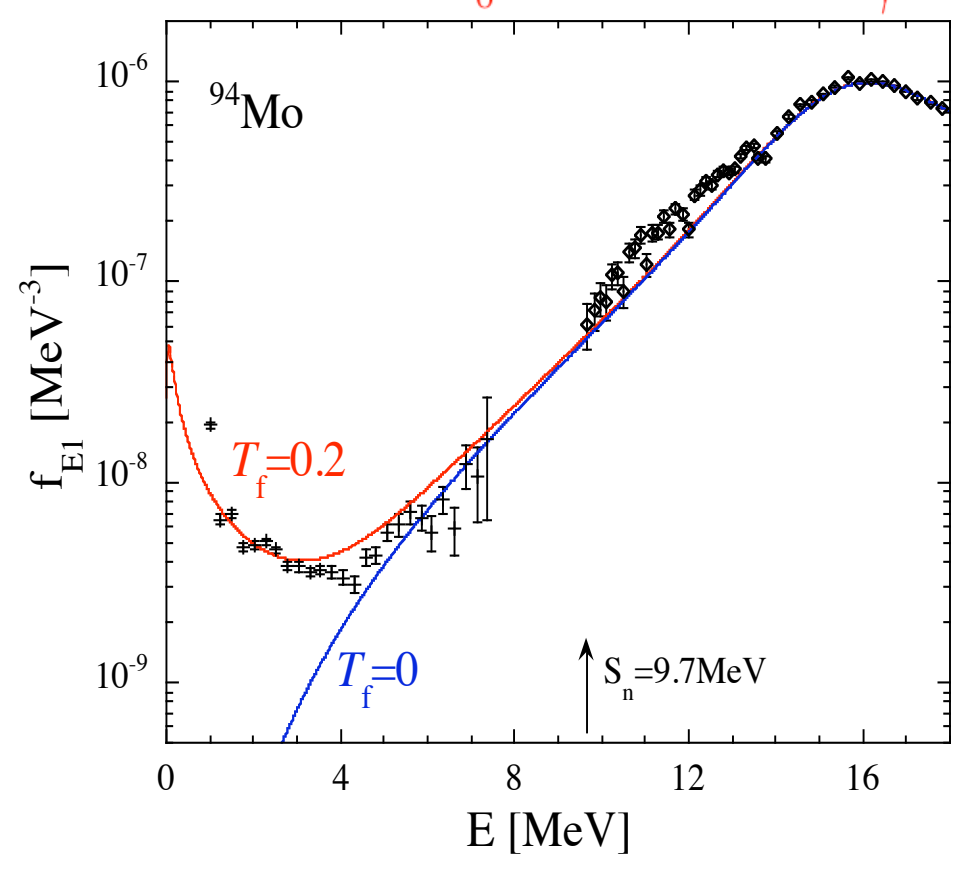




\section{Impact of the upbend pattern on the radiative n-capture rate}

Comparison of the standard GLO with GLO including upbend

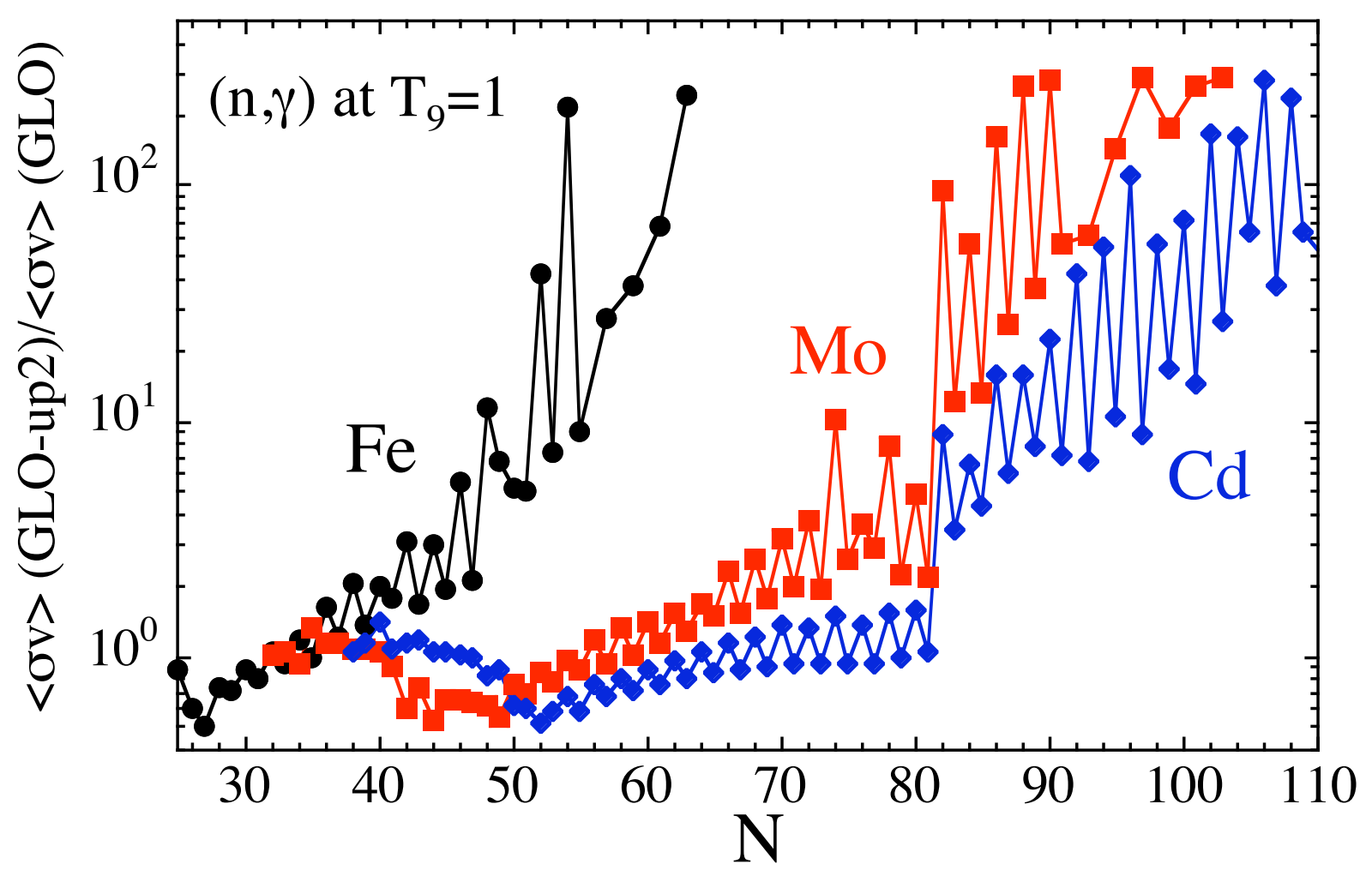

Small impact on the stable nuclei ( $\sim$ factor of 2 at most)

Large impact on exotic n-rich nuclei $\left(\mathrm{N}>\mathrm{N}_{\mathrm{mag}}\right.$ : up to a factor $\left.\sim 100\right)$

--> The upbend structure, but if true, its impact is far from being negligible 


\section{Nuclear Level Densities}




\section{Nuclear Level Densities}

Global models available for nuclear level densities:

- Macroscopic-Microscopic Approaches

\section{Reliability Accuracy}

Back-Shifted Fermi Gas model

Shell-dependent BSFG model with(out) coll. Enh.

Generalized Superfluid Model

- Semi-Microscopic Model

Statistical Model

Combinatorial Model

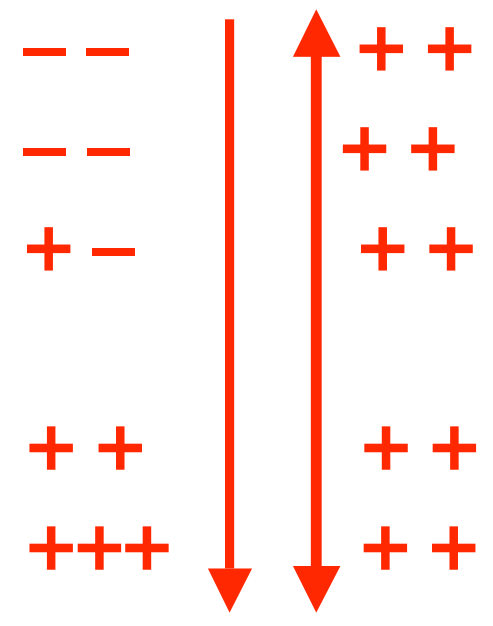

Extensive literature on microscopic models but not much of practical use for nuclear applications

Experimental constraints:

- $\sim 295$ s-wave neutron spacing at $\mathrm{U}=\mathrm{S}_{\mathrm{n}}$

- low-lying states for 1200 nuclei,

- Many model-dependent data exist [e.g Oslo data from $\left({ }^{3} \mathrm{He}, \alpha \gamma\right)$ and $\left.\left({ }^{3} \mathrm{He},{ }^{3} \mathrm{He}{ }^{\prime} \gamma\right)\right]$ 


\section{The Fermi Gas Model}

$\rho(U, J, \pi)=\frac{1}{2} \frac{\sqrt{\pi}}{12} \frac{\exp (2 \sqrt{\mathrm{aU}})}{\mathrm{a}^{1 / 4} \mathrm{U}^{5 / 4}} \frac{2 \mathrm{~J}+1}{2 \sqrt{2 \pi} \sigma_{\mathrm{M}}^{3}} \exp \left[-\frac{(\mathrm{J}+1 / 2)^{2}}{2 \sigma_{\mathrm{M}}^{2}}\right]$ with $\sigma_{\mathrm{M}}^{2}=I_{\text {rig }} \sqrt{\frac{\mathrm{U}}{\mathrm{a}}} \quad\left(I_{\text {rig }}=9.6510^{-3} \mathrm{r}_{0}{ }^{2} \mathrm{~A}^{5 / 3}\right.$ in $\hbar^{2} \mathrm{MeV}^{-1}$ units $)$

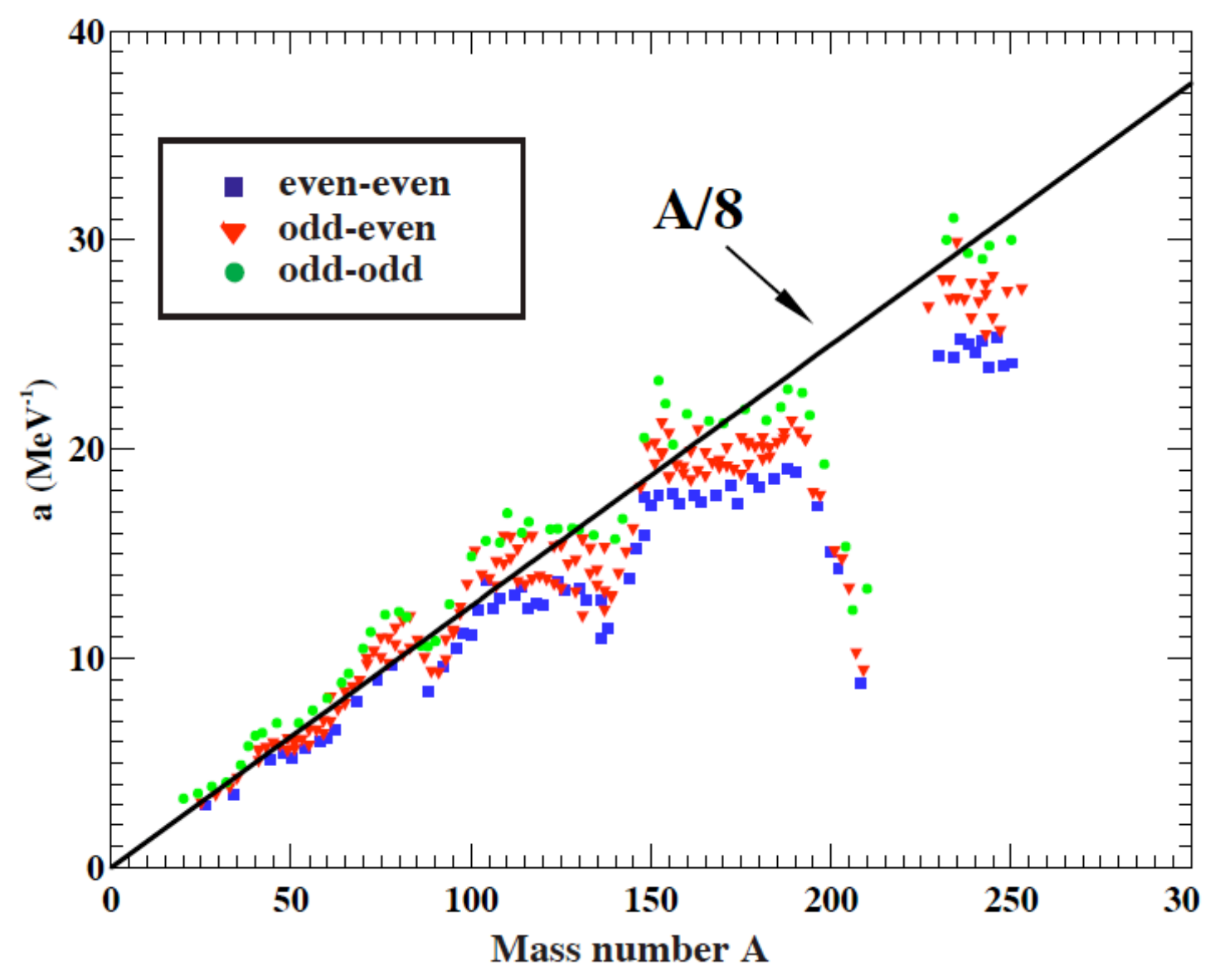


Correction for pairing correlations

One replaces $\mathbf{U}$ by $\mathbf{U}^{*}=\mathbf{U}-\chi \Delta_{0}$ with $\Delta_{0}=\frac{\mathbf{1 2}}{\sqrt{\mathbf{A}}}$ and $\chi=0,1$ or 2 for odd-odd, odd-even or even-even nuclei ( $\Delta_{0}=$ average energy necessary to break a pair of nucleons)

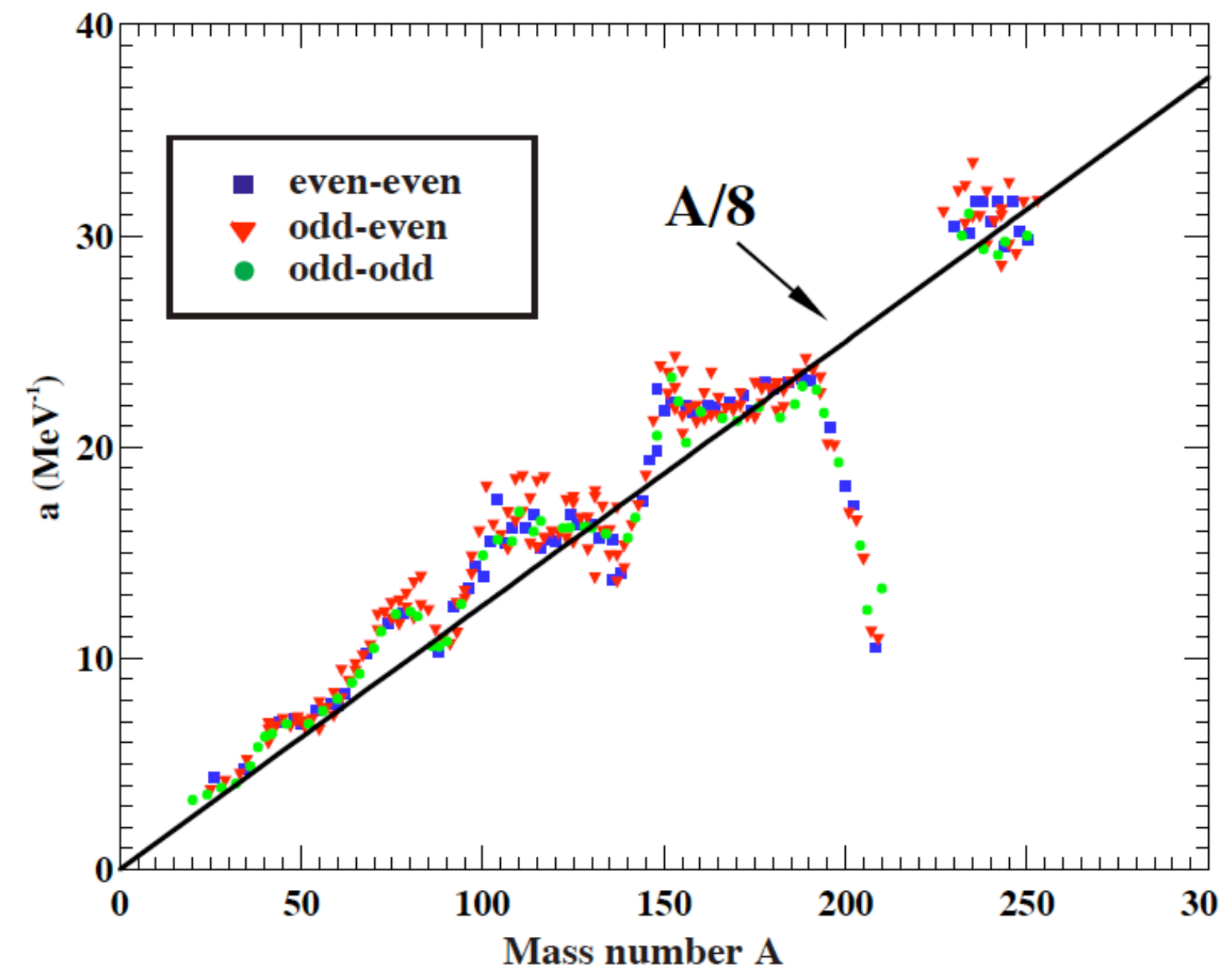


The Back-Shifted Fermi Gas model with U-dependent shell effect

$$
\mathbf{a}(\mathbf{N}, \mathbf{Z}, \mathbf{U})=\tilde{\mathbf{a}}(\mathbf{A})\left[1+\delta \mathbf{W}(\mathbf{N}, \mathbf{Z}) \frac{1-\exp (-\gamma \mathbf{U})}{\mathbf{U}}\right]
$$

$$
\text { with } \tilde{\mathbf{a}}(\mathbf{A})=\alpha \mathbf{A}+\beta \mathbf{A}^{2} \text { and } \delta \mathbf{W}(\mathbf{N}, \mathbf{Z})=\mathbf{M}_{\exp }-\mathbf{M}_{l d}
$$
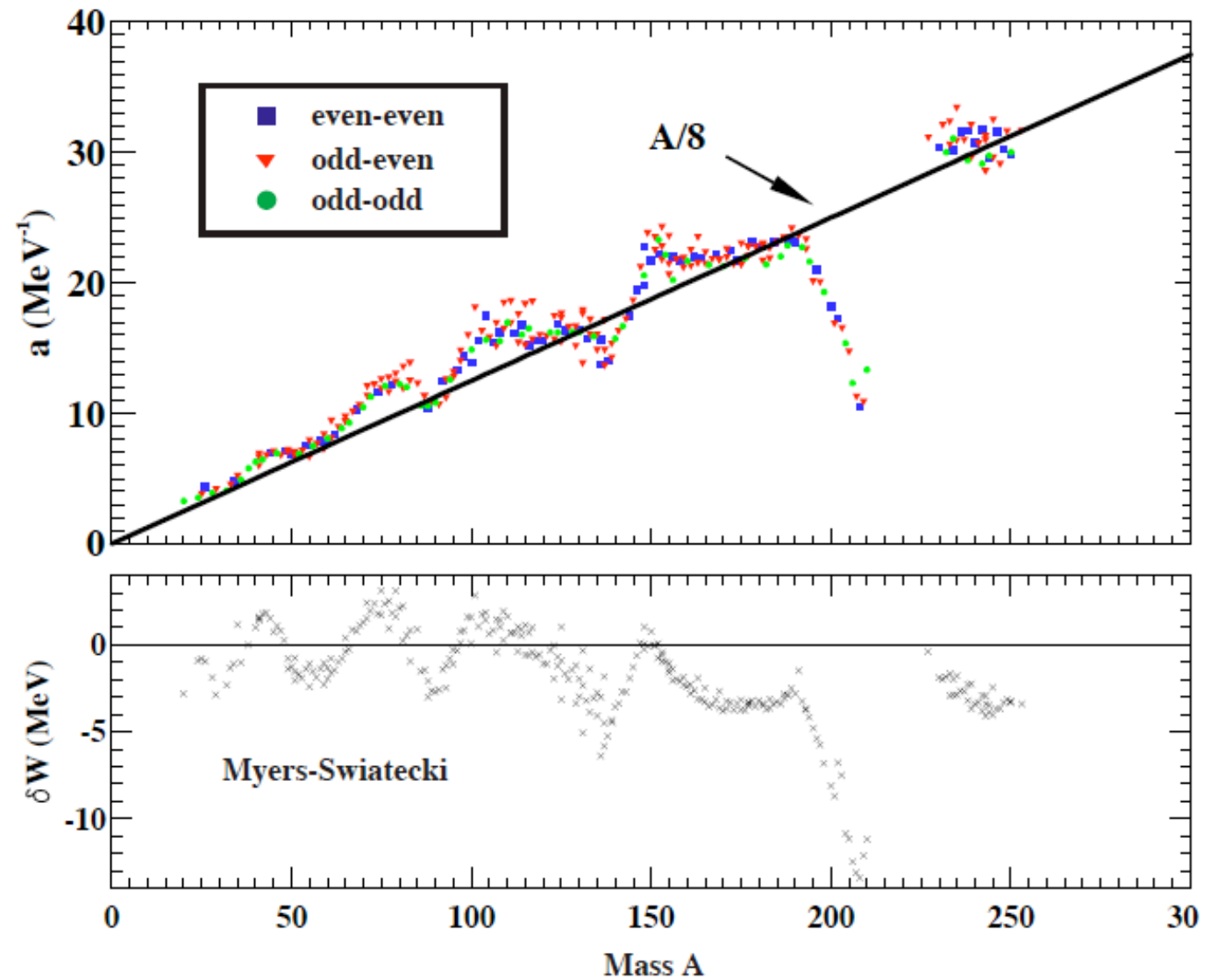


\section{Global microscopic NLD formula}

- NLD formula within the statistical (partition function) method based on the HFBCS (MSk7) ground-state properties

- Single particle level scheme

- Ground-state deformation parameters and energy

- Pairing strength (though renormalized consistently)

- Microscopic NLD formula includes

- Shell correction inherent in the mean field s.p. level scheme

- Pairing correction (in the constant-G approximation) with blocking effects

- Spin-dependence with microscopic shell and pairing effects

- Deformation effects included in

- the single-particle level scheme

- the collective contribution of the rotational band on top of each intrinsic state

- disappearance of deformation effects at increasing excitation energies

$\rightarrow$ Reliability: Accuracy:
Exact solution the analytical formulas tries to mimic Competitive with parametrized formulas in reproducing experimental data 


\section{Comparison with experimental neutron resonance spacings}
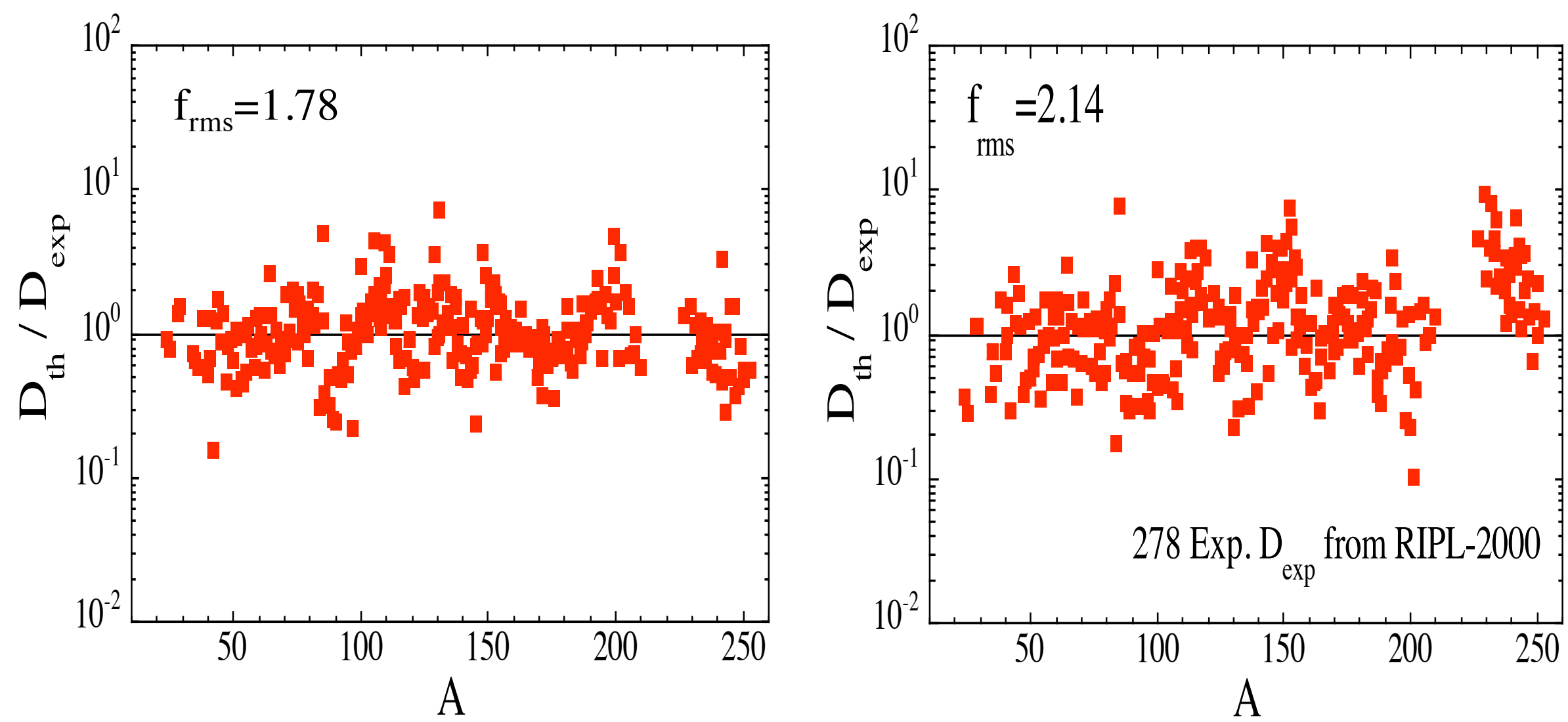


\section{Comparison of NLD predictions}

Microscopic NLD formula based on HF-BCS

VS

Analytical shell-corrected Back-Shifted Fermi Gas

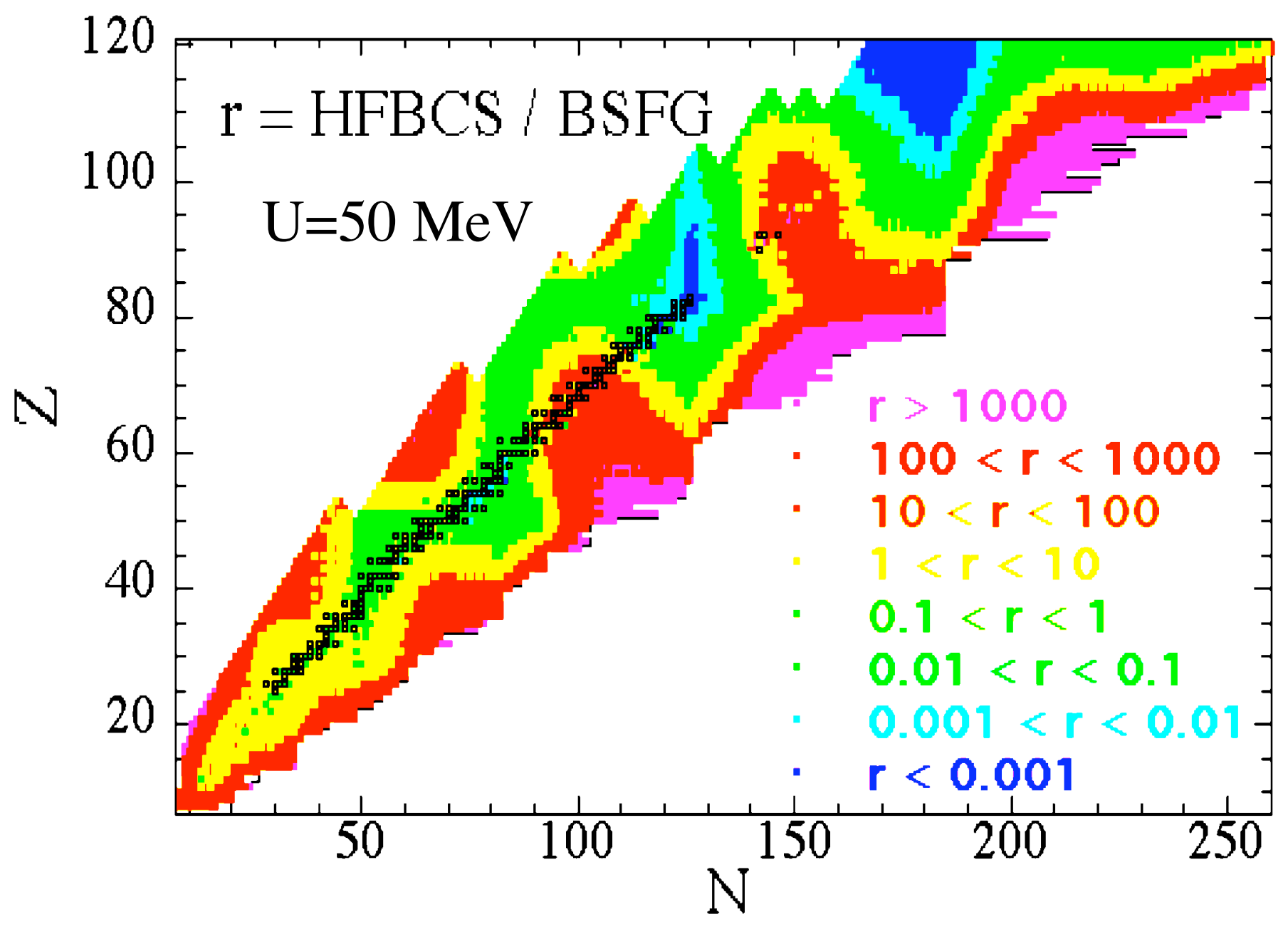




\section{Global combinatorial NLD formula}

Level density estimate is a counting problem: $\quad \rho(U)=\mathrm{d} N(U) / \mathrm{d} U$

$N(U)$ is the number of ways to distribute the nucleons among the available levels for a fixed excitation energy $U$

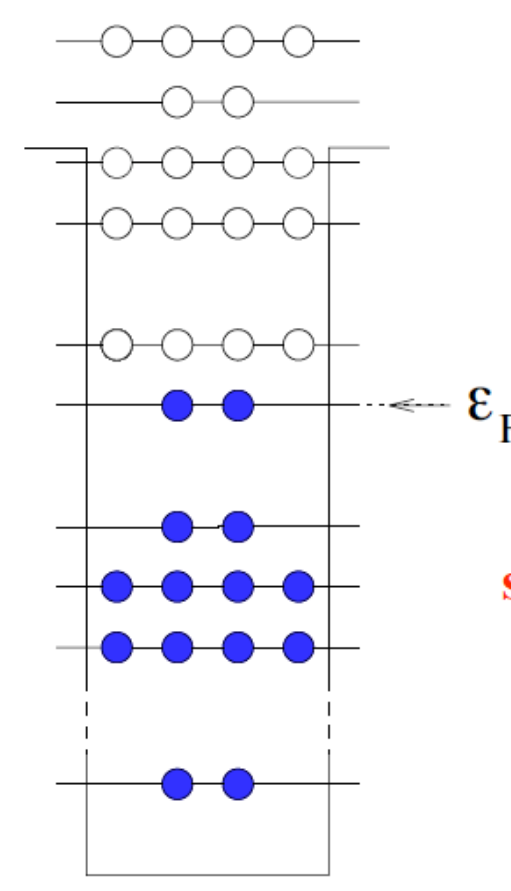

ground state

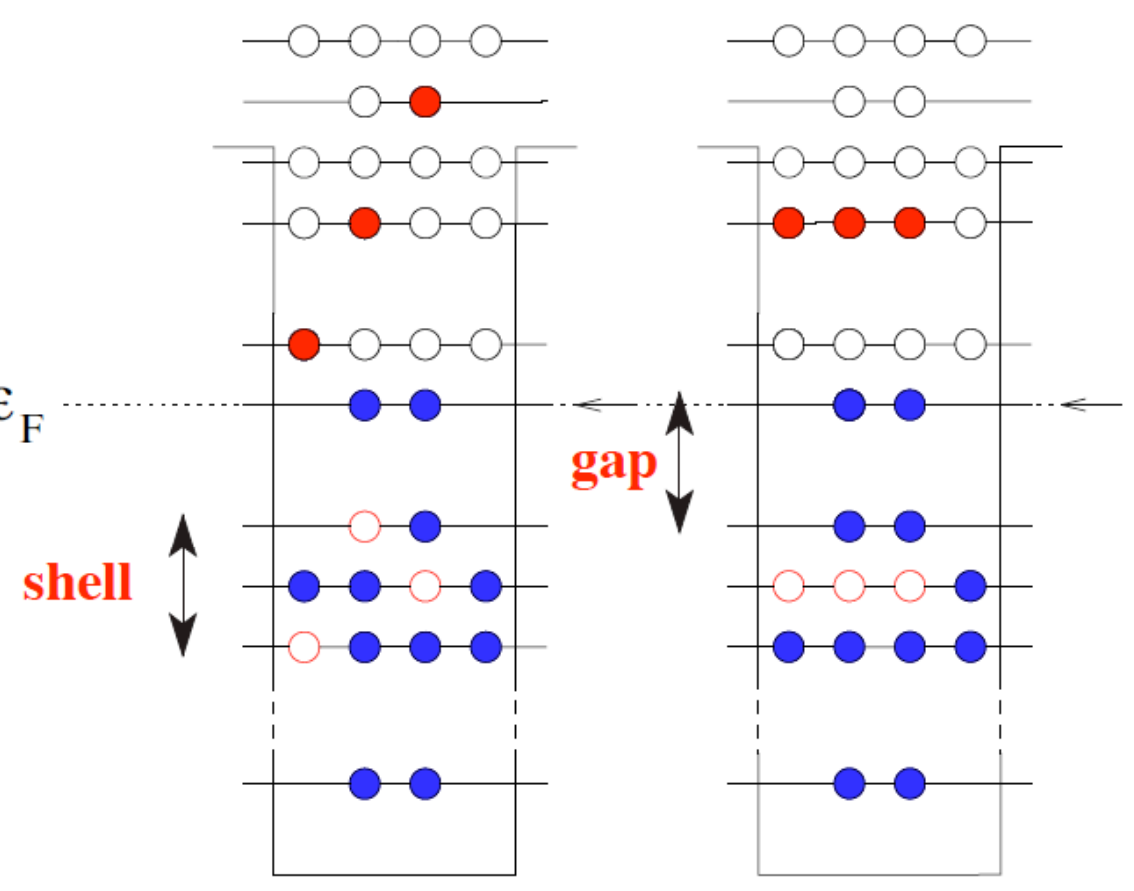

excited levels

(3 particles - 3 holes) 


\section{Global combinatorial NLD formula}

- Ground-state properties obtained within HFB with the BSk14 Skyrme force (force fitted to 2149 exp. nuclear masses with $\sigma=0.730 \mathrm{MeV}$ )

- Single particle level scheme

- Pairing strength (consistency between BSk14 and experimental pairing gaps)

- NLD formula within the combinatorial method (Hilaire 2006)

- Parity, angular momentum, pairing correlations, shell effect and rotational and vibrational enhancement treated explicitely and coherently

- Inclusion of phenomenological corrections for disappearance of deformation effects at increasing excitation energies

Global combinatorial calculations of practical use in applications

- Particle-hole as well as total parity-, spin- and E-dependent NLD

- Deviation from the statistical limit at low energies (discrete counting) 


\section{Comparison with experimental neutron resonance spacings}

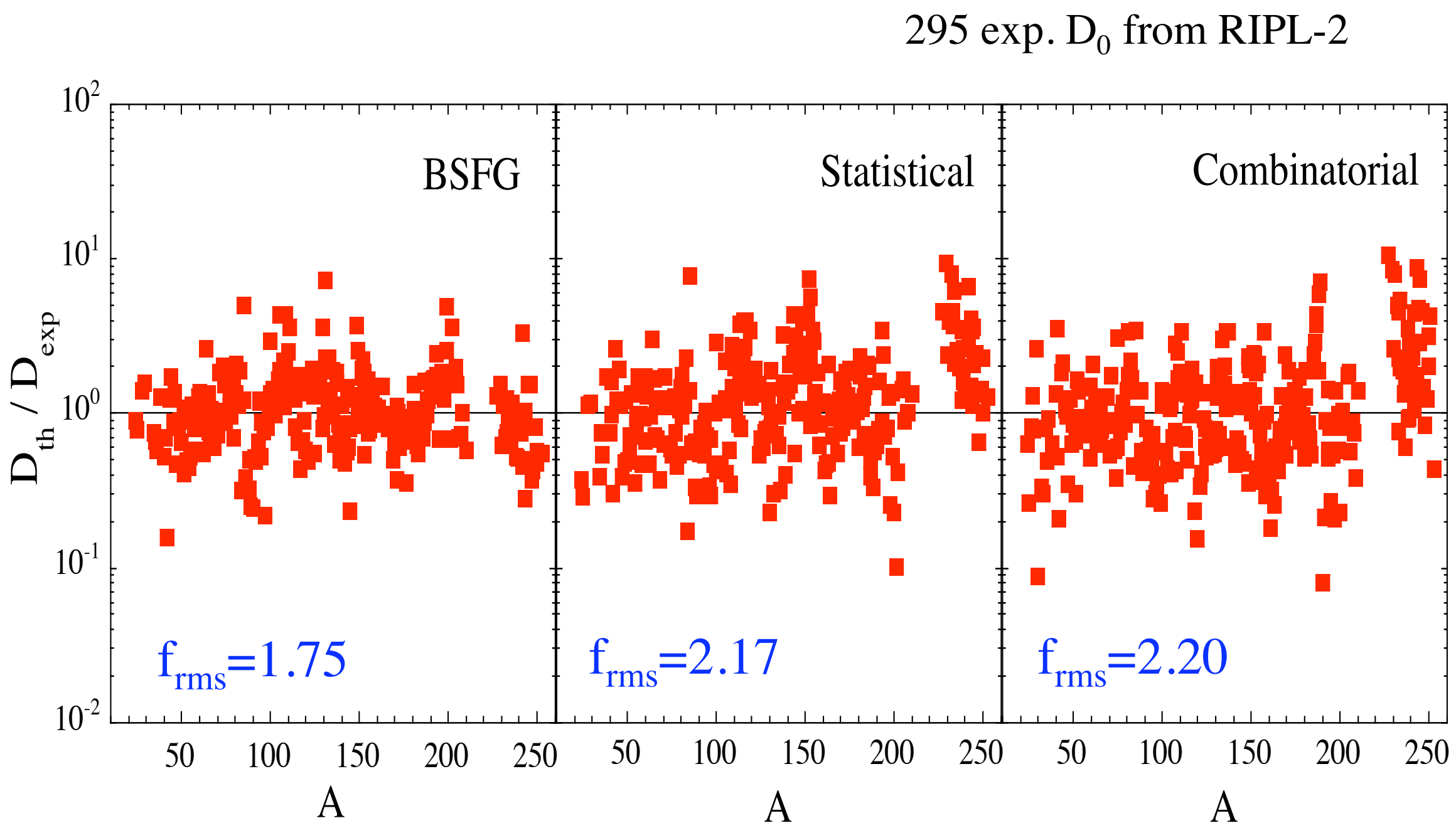




\section{Spin dependence}

Non-statististical spin dependence in the combinatorial level densities

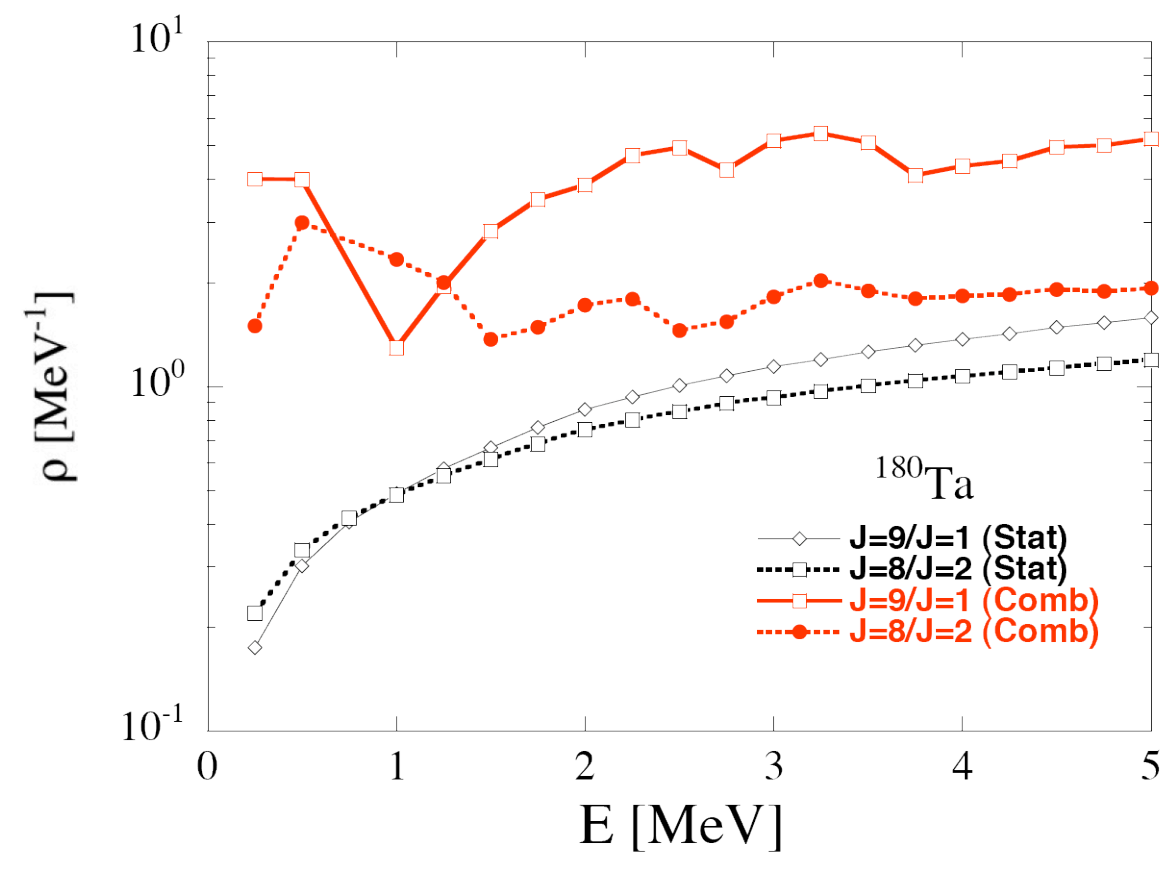

Impact on the photoneutron cross sections for the isomeric state ${ }^{180} \mathrm{Ta}^{\mathrm{m}}$

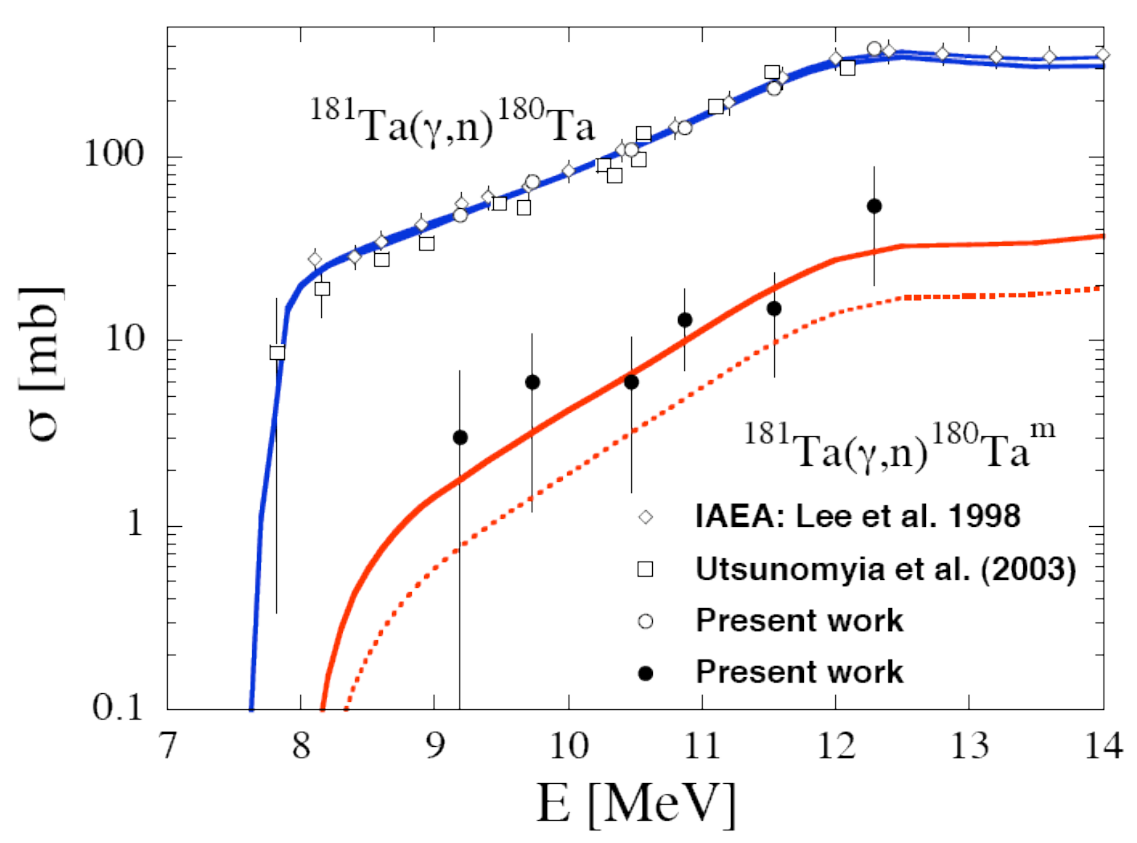




\section{Parity-spin dependence}

Simple (spin-independent) approaches can not account for the complexity of the parity dependence.
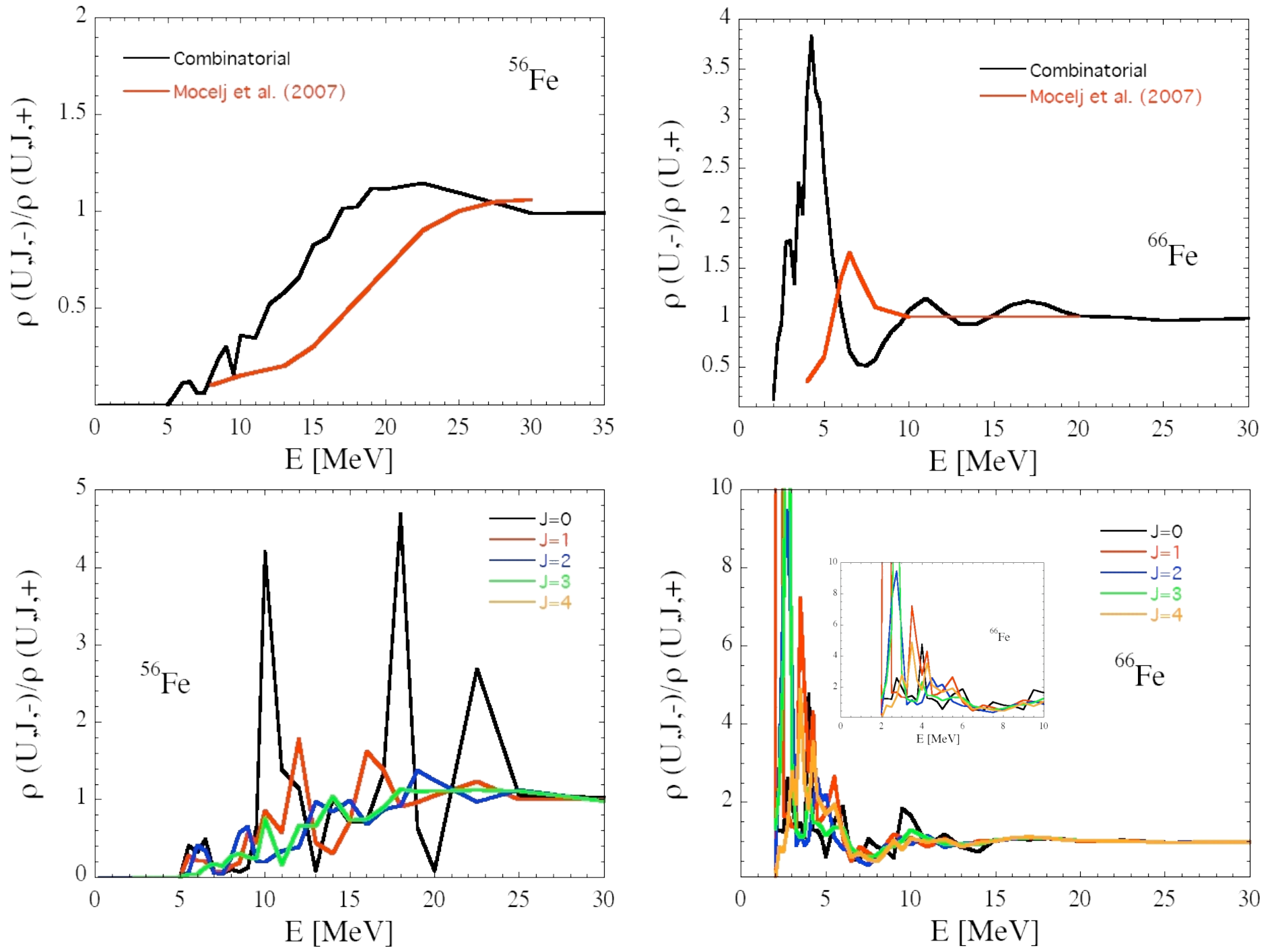
Impact of different NLD models on the radiative n-capture rate
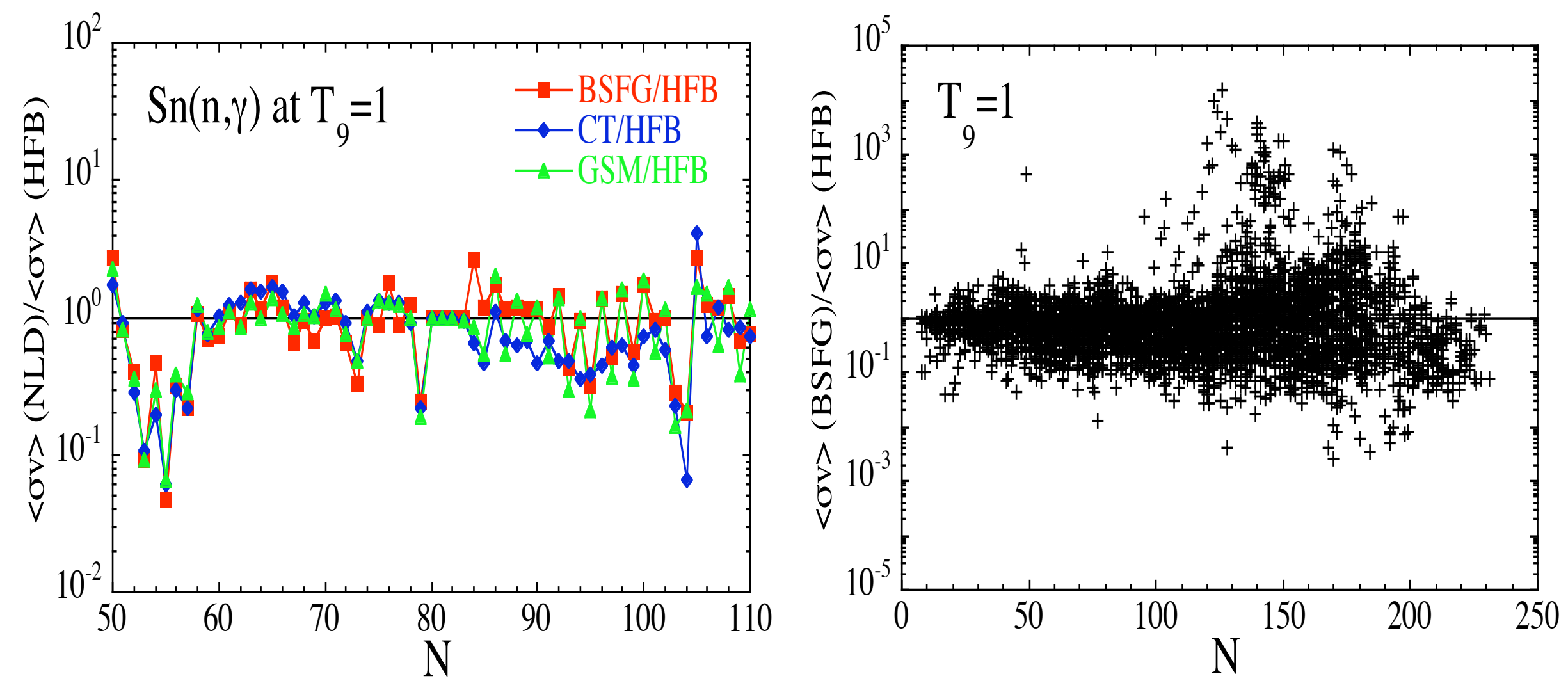

P.S. BSFG, CT and GSM models are shell-dependent 


\section{Relevance of the Combinatorial approach for the DC cross section}

Unknown levels estimated with a Combinatorial NLD:

- 1 neutron $\mathrm{p}$-h excitations $\left(C^{2} S=1\right)$

- full intrinsic (all n-p ph excitations) NLD with an average $\left\langle C^{2} S\right\rangle=0.5$

$$
\sigma^{D C}(E)=\sum_{f=0}^{x} C_{f}^{2} S_{f} \sigma_{f}^{D C}(E)+\sum_{E_{f}, J_{f}, \pi_{f}}\left\langle C^{2} S\right\rangle \mathcal{N}\left(E_{f}, J_{f}, \pi_{f}\right) \sigma_{f}^{D C}(E)
$$

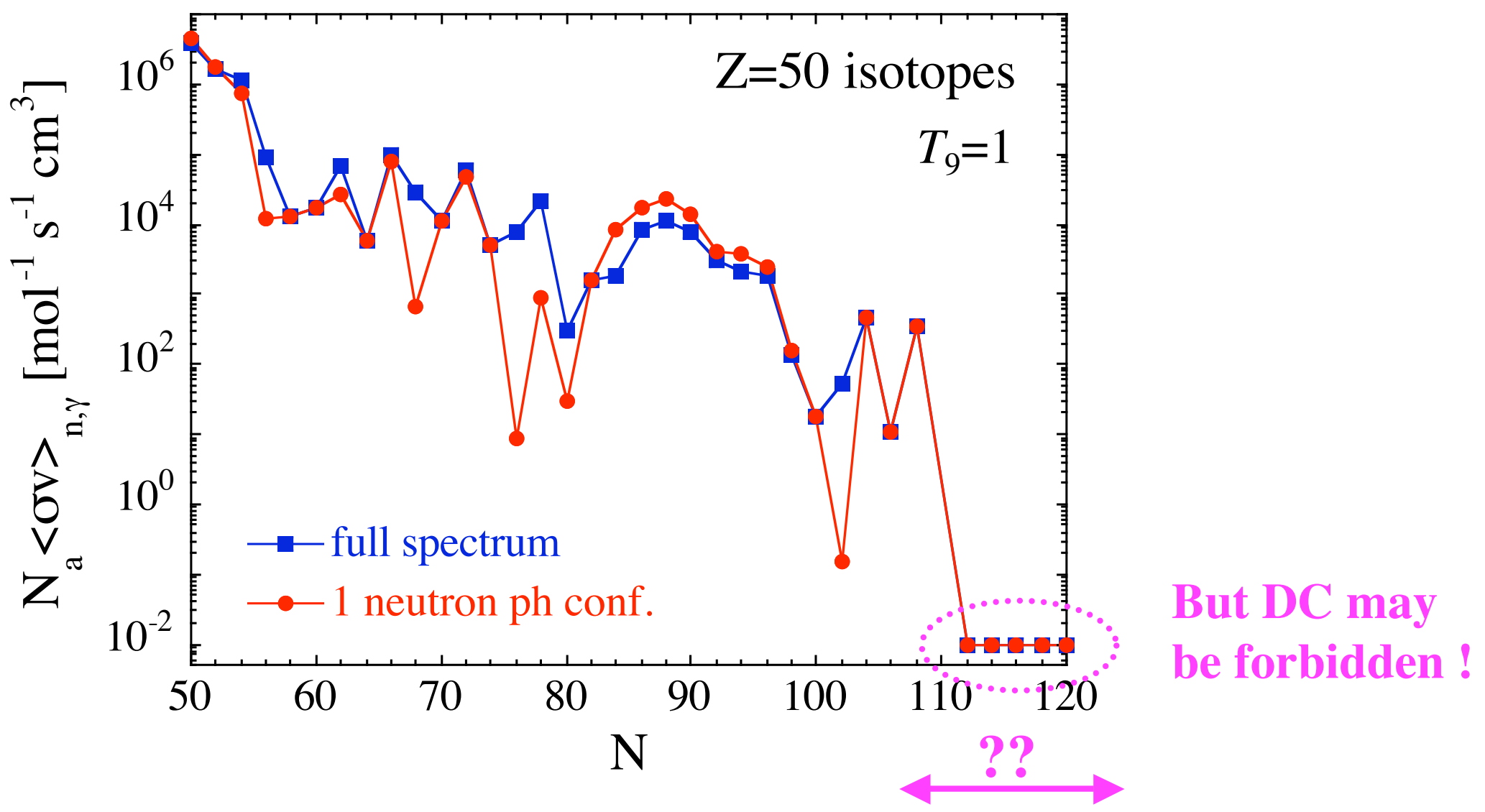




\section{The optical model potential}




\section{Optical Model Potential}

Global models available for optical model potentials:

- Phenomenological approaches

Reliability Accuracy

- Semi-Microscopic Model

Woods-Saxon potential

Volume \& Surface WS potential

Coupled-channel WS potential with dispersive rel.

Folding potential

BHF-type potential

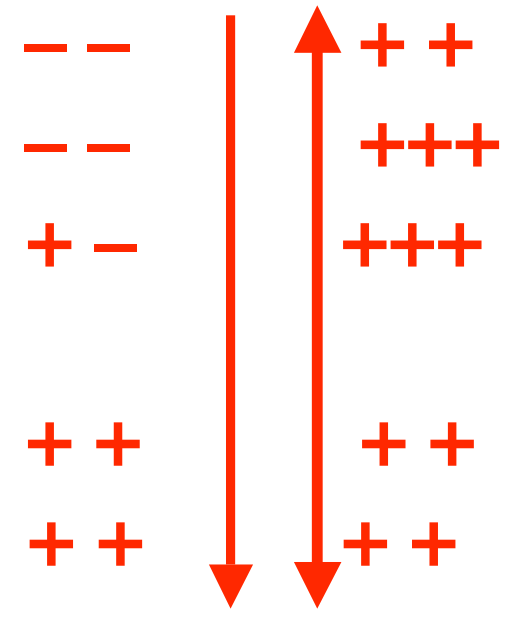

Most of reaction rate calculations for astrophysics applications use the spherical JLM-type potential 


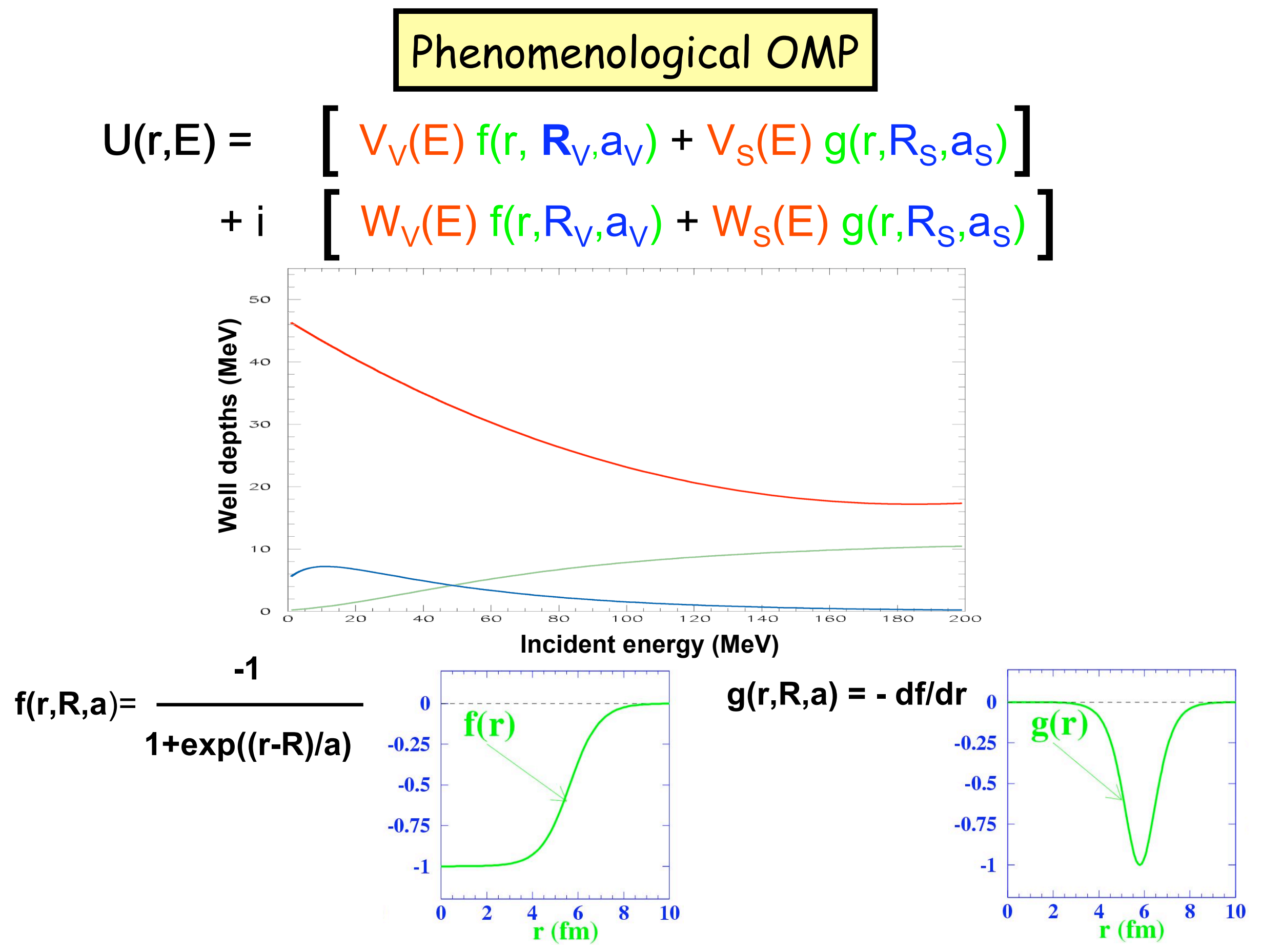




\section{Phenomenological OMP}

$\sim 20$ adjusted parameters Very accurate (1\%)

relatively weak predictive power far away from stability

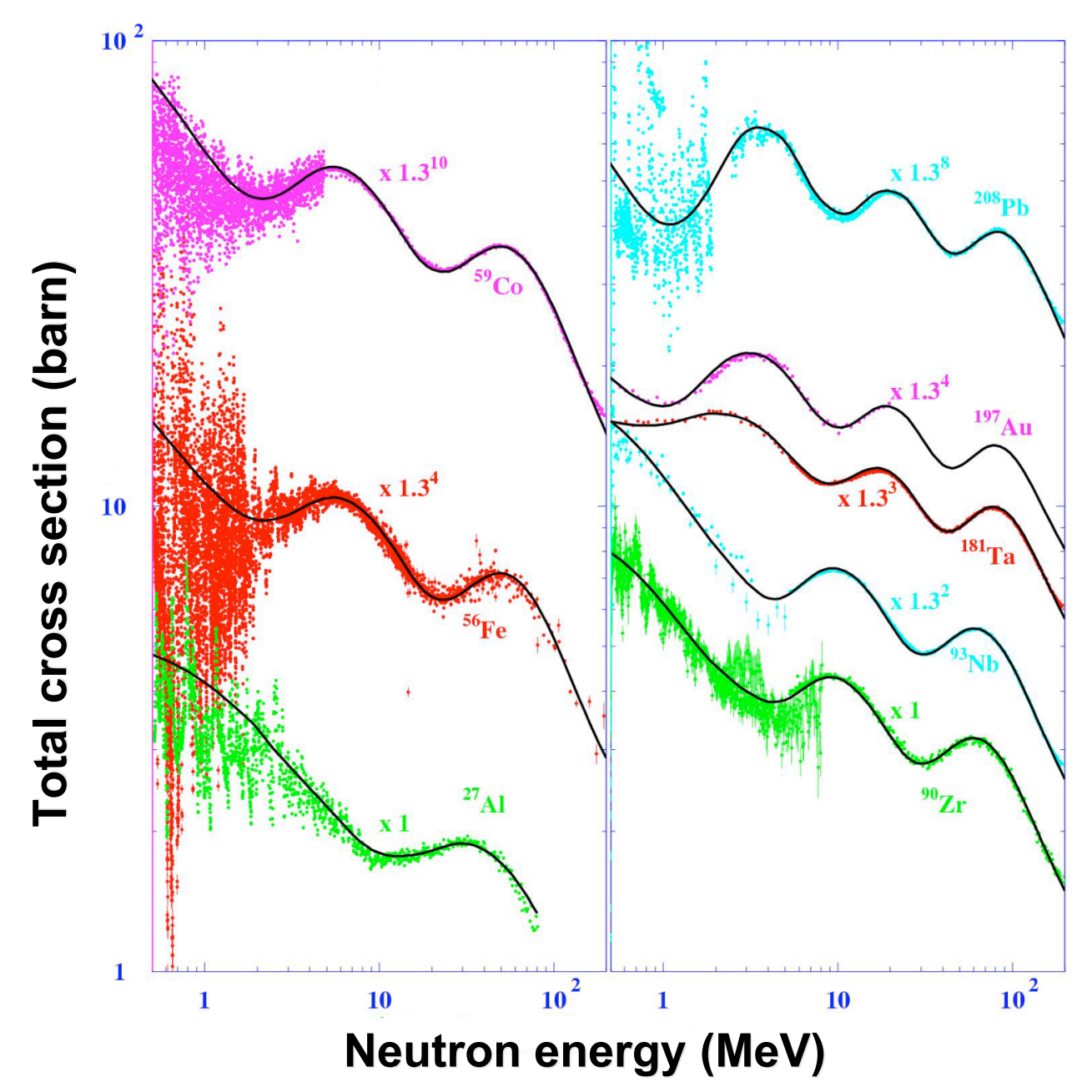




\section{Semi-microscopic OMP}

\section{Optical potential $=\quad \begin{gathered}\text { Effective } \\ \text { Interaction }\end{gathered}$}

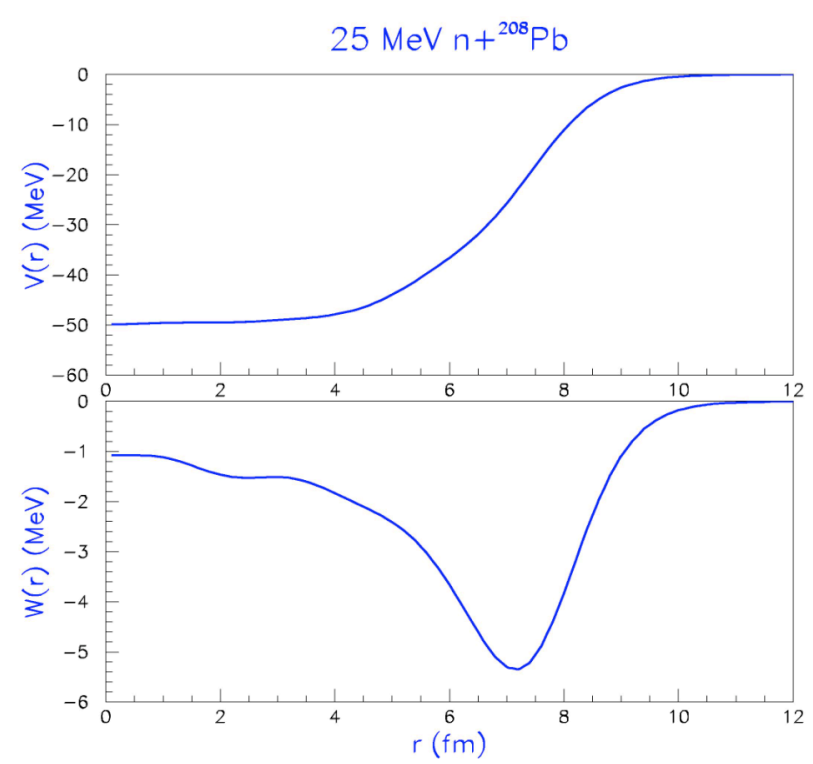

$$
\mathrm{U}(r, E)
$$

Depends on the nucleus

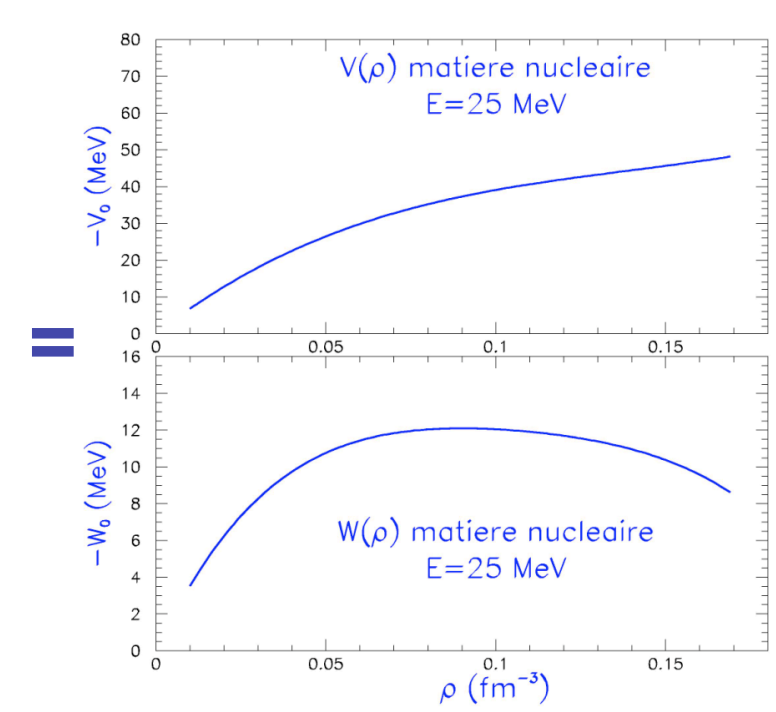

$=\frac{\mathrm{U}\left(\rho\left(r^{\mathrm{s}}\right), \mathrm{E}\right)}{\rho\left(\mathrm{r}^{\mathrm{P}}\right)}$

Independent of the nucleus $\otimes \quad$ Radial densities

$\otimes$

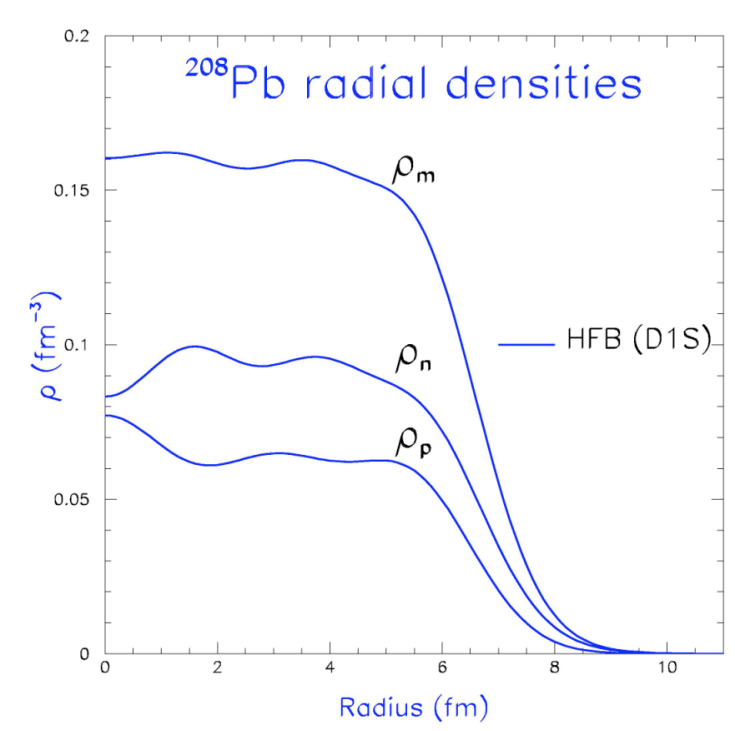

$\otimes$

$$
\rho(r)
$$

Depends on the nucleus 


\section{Semi-microscopic OMP}

Jeukenne-Lejeune-Mahaux (JLM) potential

- Real part:

- Imaginary part:
$V(r, E)=\left[V_{0}(r, E) \pm\right.$ $W(r, E)=\left[W_{0}(r, E) \pm\left(\rho_{n}-\rho_{p}\right) /\left(\rho_{n}+\rho_{p}\right) W_{1}(r, E)\right]$ - protons

- No adjustable parameters

- Based on nuclear structure properties

$\Rightarrow$ usable for any nucleus

- Less precise than the phenomenological approach

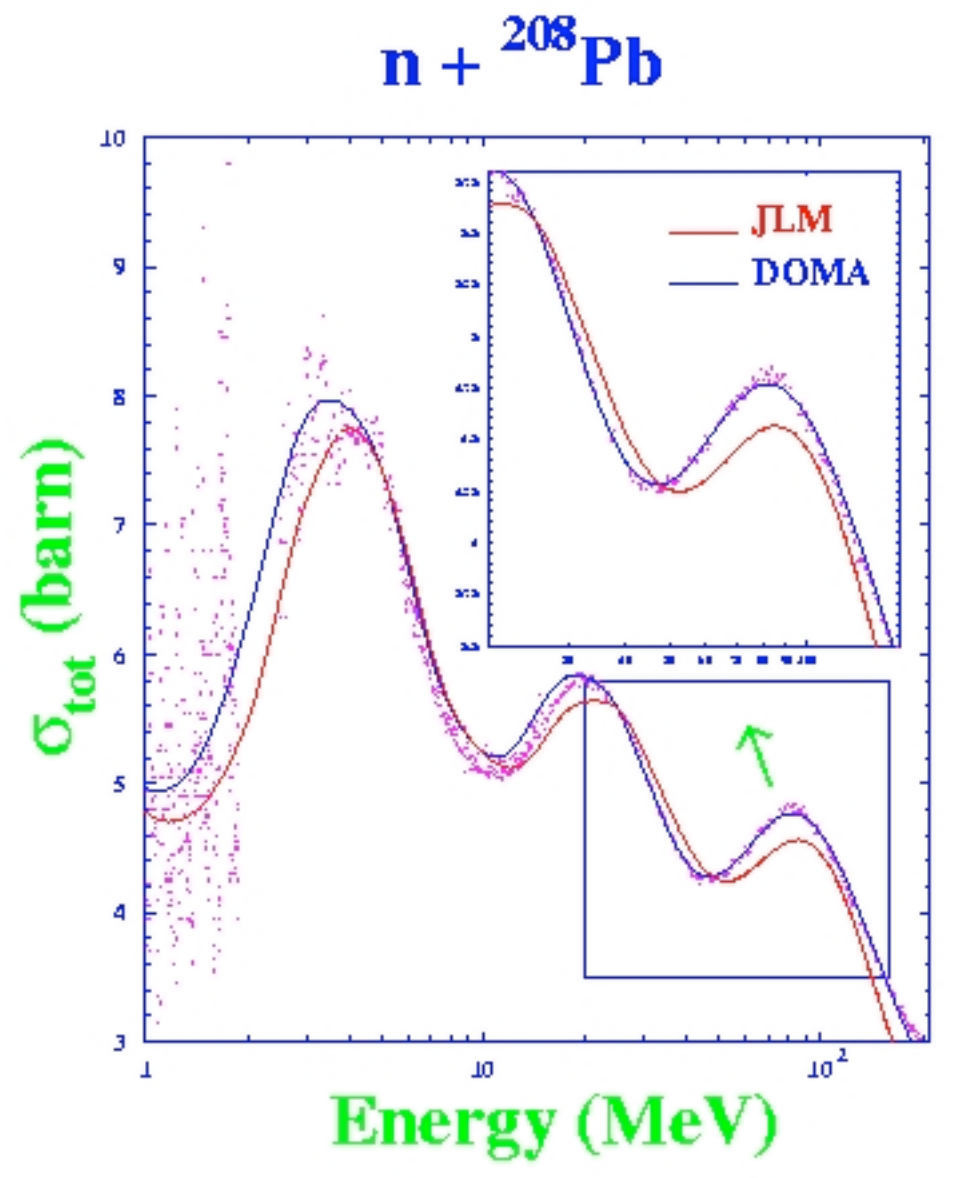




\section{The JLM-Bruyères (JLMB) potential}

Renormalization of the JLM potential in a Lane-consistent potential (isospin symmetric)

- Real part: $\mathrm{V}(\mathrm{r}, \mathrm{E})=\lambda_{\mathrm{v}}\left[\mathrm{V}_{0}(\mathrm{r}, \mathrm{E}) \pm \lambda_{\mathrm{v} 1}\left(\rho_{\mathrm{n}}-\rho_{\mathrm{p}}\right) /\left(\rho_{\mathrm{n}}+\rho_{\mathrm{p}}\right) \mathrm{V}_{1}(\mathrm{r}, \mathrm{E})\right]$

+ neutrons

- Imaginary part: $W(r, E)=\lambda_{w}\left[W_{0}(r, E) \pm \lambda_{w 1}\left(\rho_{n}-\rho_{p}\right) /\left(\rho_{n}+\rho_{p}\right) W(r, E)\right]$

- protons

Renormalization on $(n, n),(p, p)$ elastic scattering and $(p, n)$ QE scattering from GS to IAS as well as reaction data $(40 \leq \mathrm{A} \leq 209 ; \mathrm{E}=1 \mathrm{keV}-200 \mathrm{MeV}) \rightarrow$ JLM-Bruyeres $(\mathrm{JLMB})$ OMP
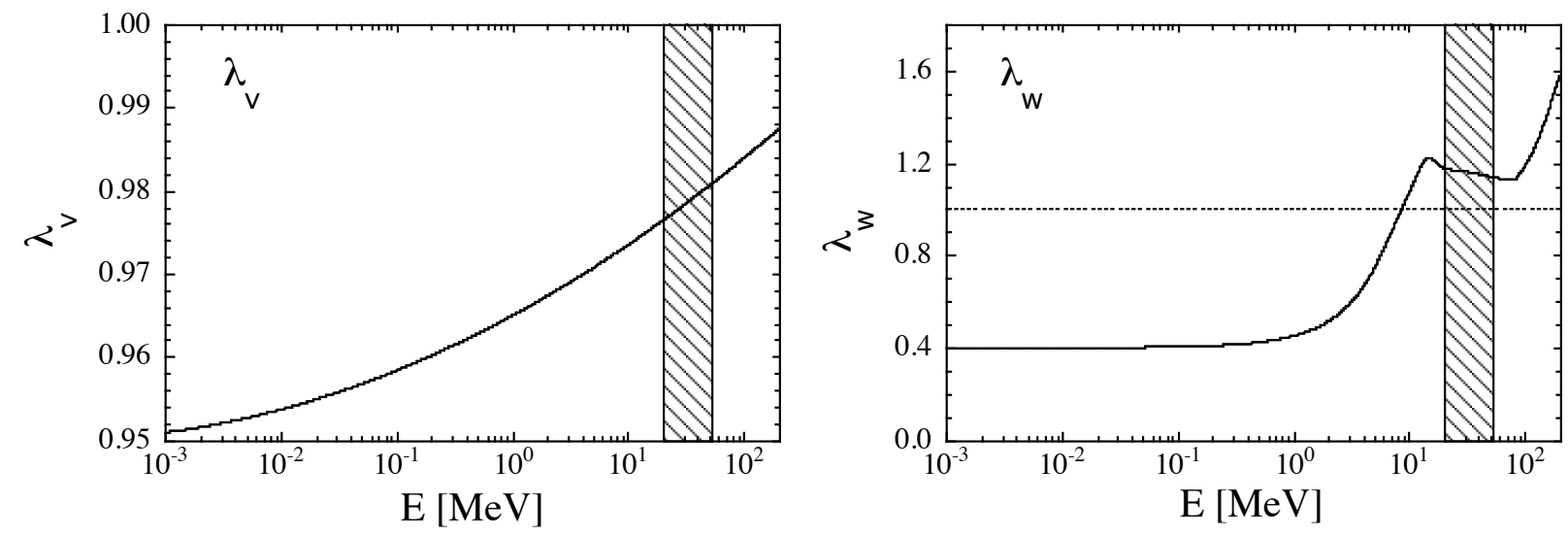

Energy region $20-50 \mathrm{MeV}$ of highest confidence: uncertainties $\sim 1.5 \%$
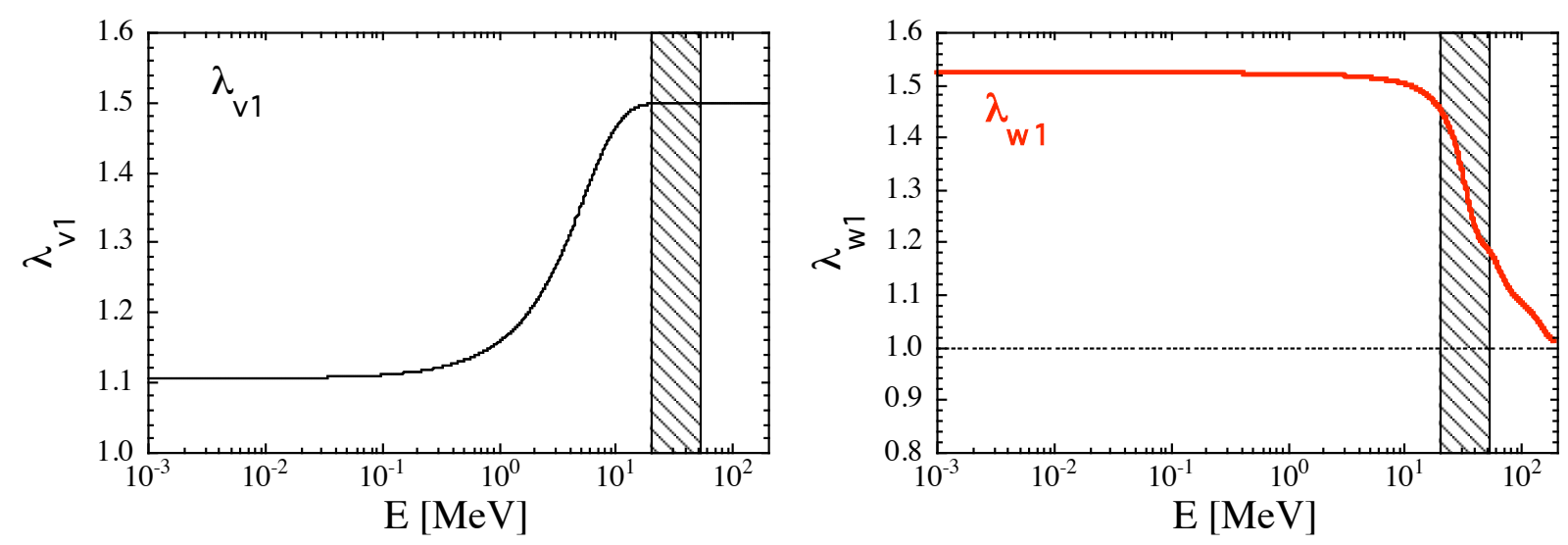


\section{Semi-microscopic OMP}

Unique description of elastic scattering $(n, n),(p, p)$ et $(p, n)$
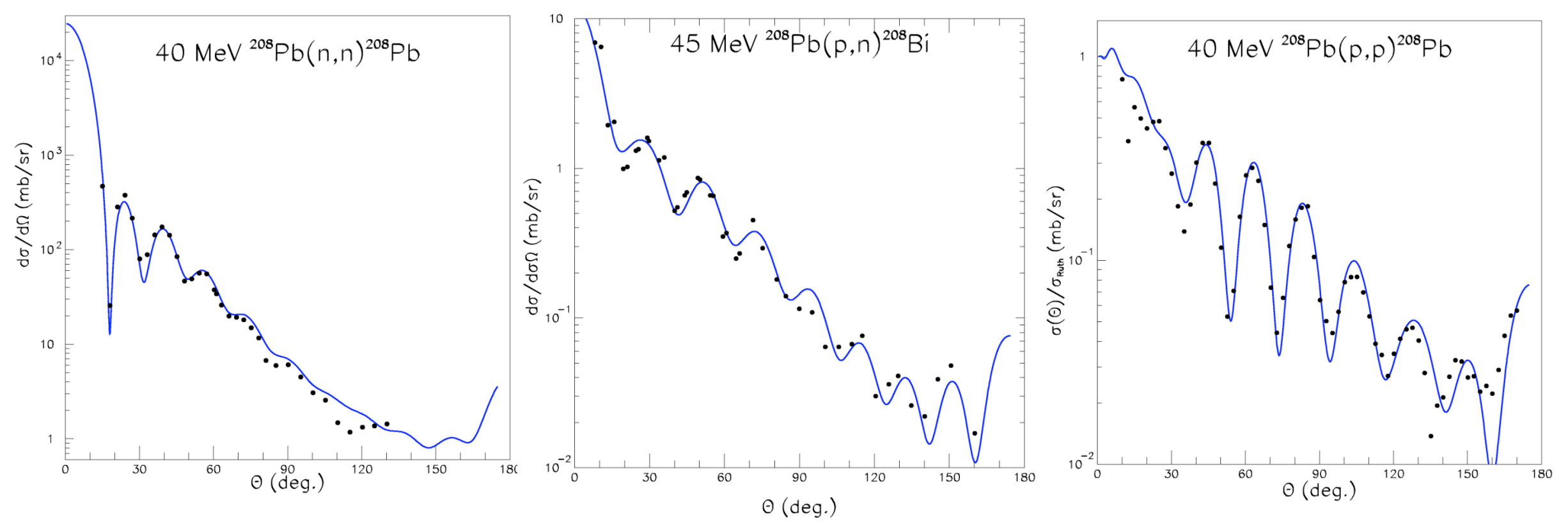


\section{Semi-microscopic OMP}

Enables to perform predictions for very exotic nuclei for which There exist no experimental data
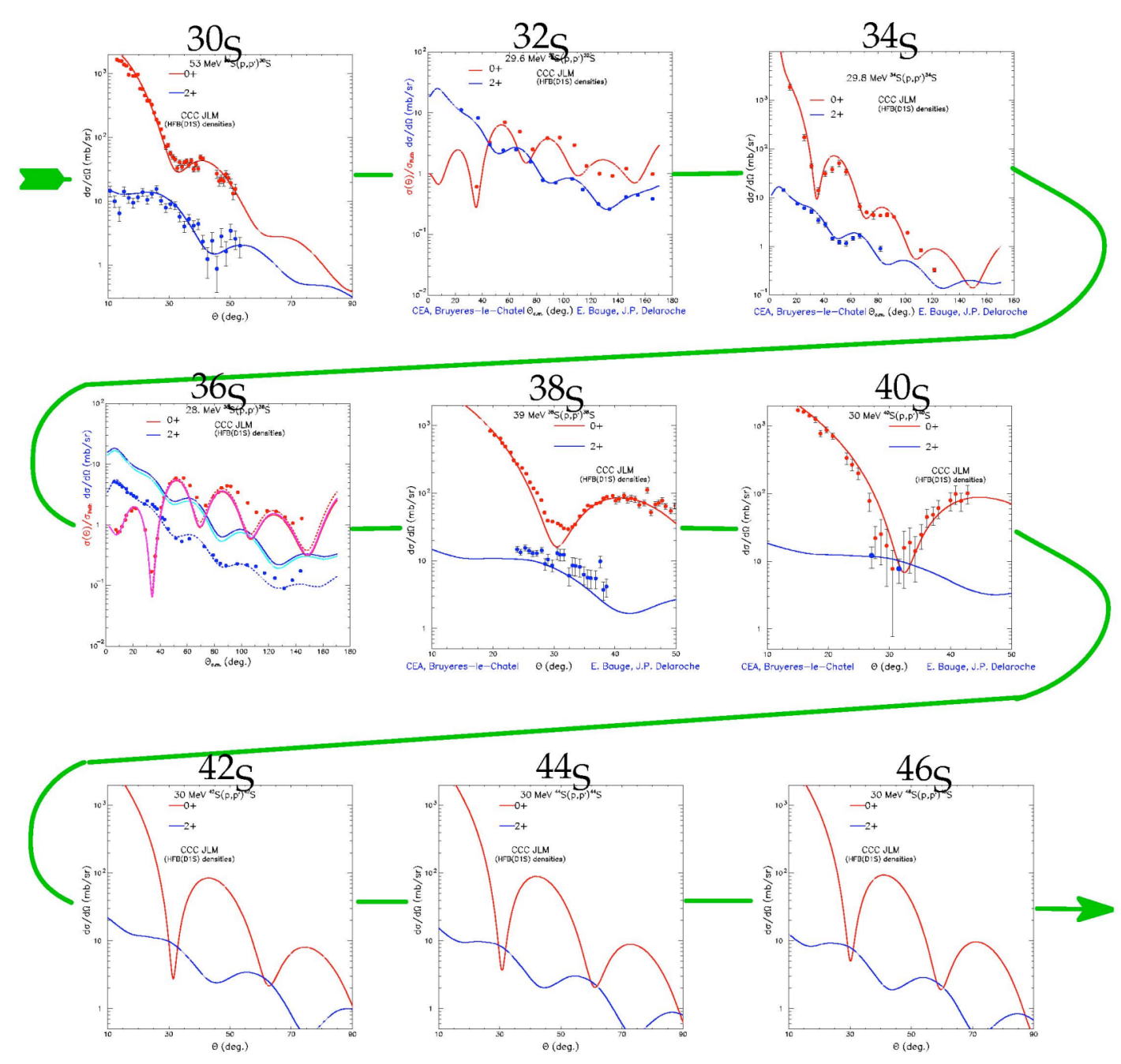


\section{The isovector imaginary neutron potential}

Renormalization of the JLM potential in a Lane-consistent potential (isospin symmetric)

- Real part: $\quad \mathrm{V}(\mathrm{r}, \mathrm{E})=\lambda_{\mathrm{v}}\left[\mathrm{V}_{0}(\mathrm{r}, \mathrm{E}) \pm \lambda_{\mathrm{v} 1}\left(\rho_{\mathrm{n}}-\rho_{\mathrm{p}}\right) /\left(\rho_{\mathrm{n}}+\rho_{\mathrm{p}}\right) \mathrm{V}_{1}(\mathrm{r}, \mathrm{E})\right]$

+ neutrons

- Imaginary part: $W(r, E)=\lambda_{w}\left[W_{0}(r, E) \pm \lambda_{w 1}\left(\rho_{n}-\rho_{p}\right) /\left(\rho_{n}+\rho_{p}\right) W W_{1}(r, E)\right]$

- protons

Renormalization on $(n, n),(p, p)$ elastic scattering and $(p, n)$ QE scattering from GS to IAS as well as reaction data $(40 \leq A \leq 209 ; E=1 \mathrm{keV}-200 \mathrm{MeV}) \rightarrow$ JLM-Bruyere (JLMB) OMP
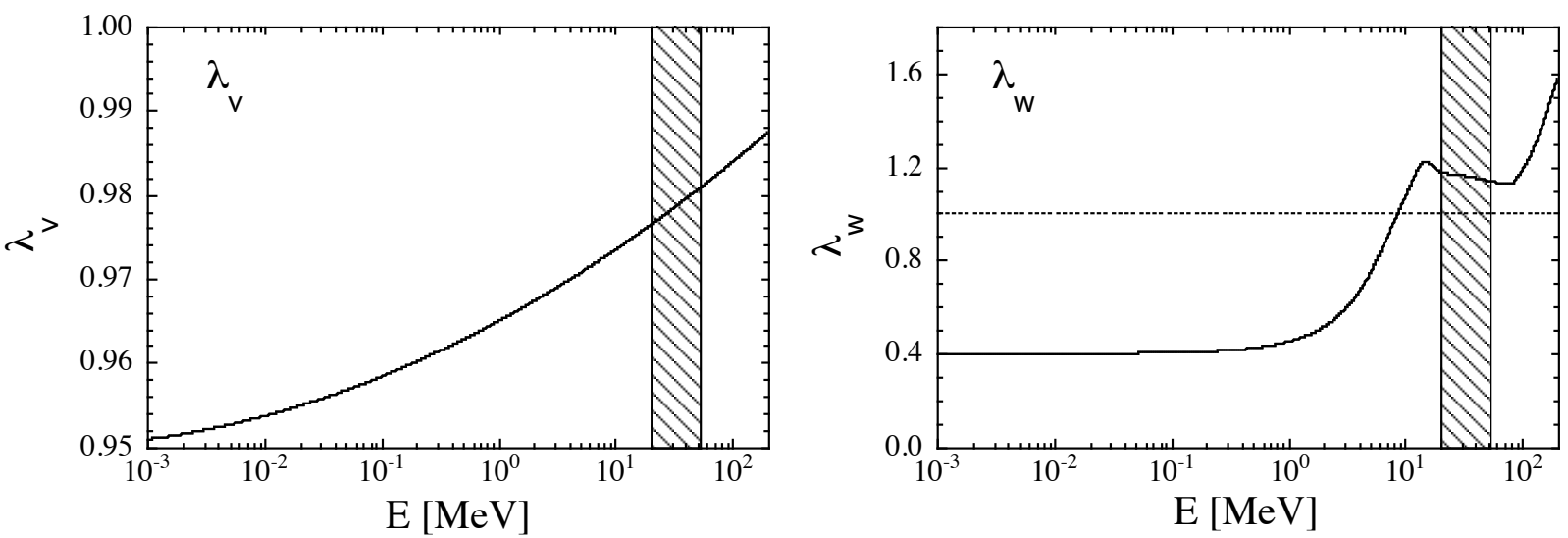

Energy region 20-50 MeV of highest confidence: uncertainties $\sim 1.5 \%$
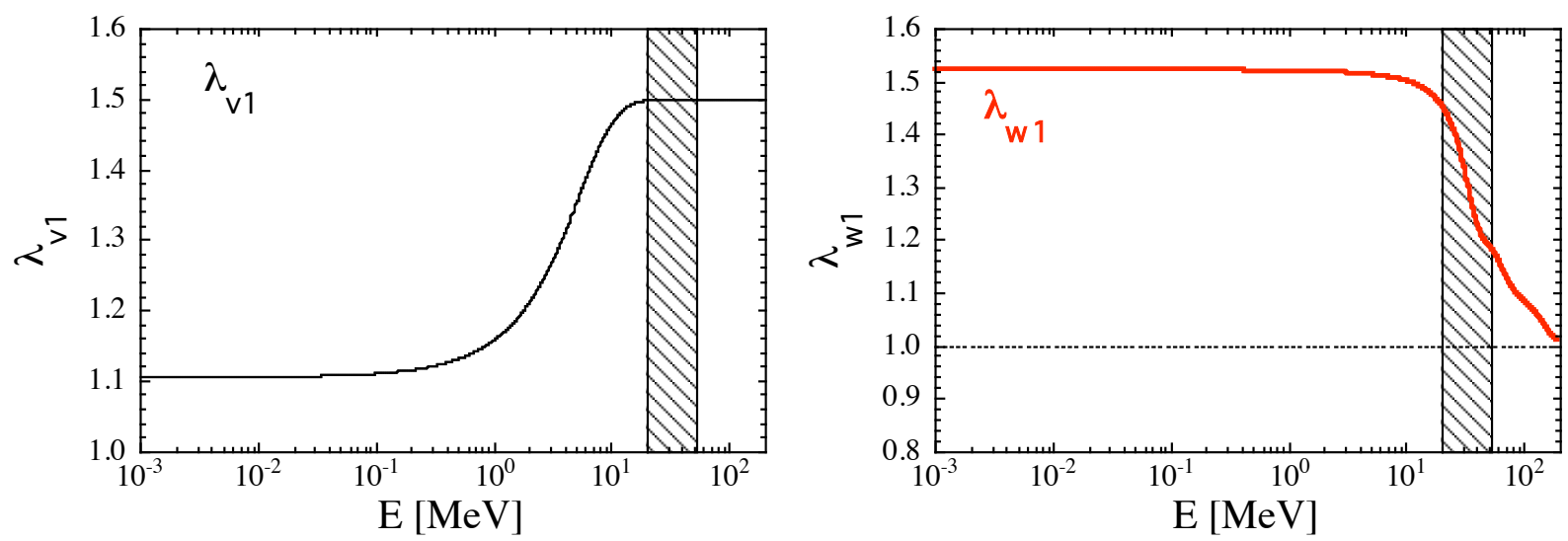

$$
\lambda_{\mathrm{w} 1}=\mathbf{1 . 5 2}
$$

at low energies but without constraint compared to $\lambda_{\mathrm{w} 1}=1$ for JLM 
Some low-energy data can be used to constrain the isovector imaginary potential:

the neutron strength function $S_{0}$ and $S_{1}$
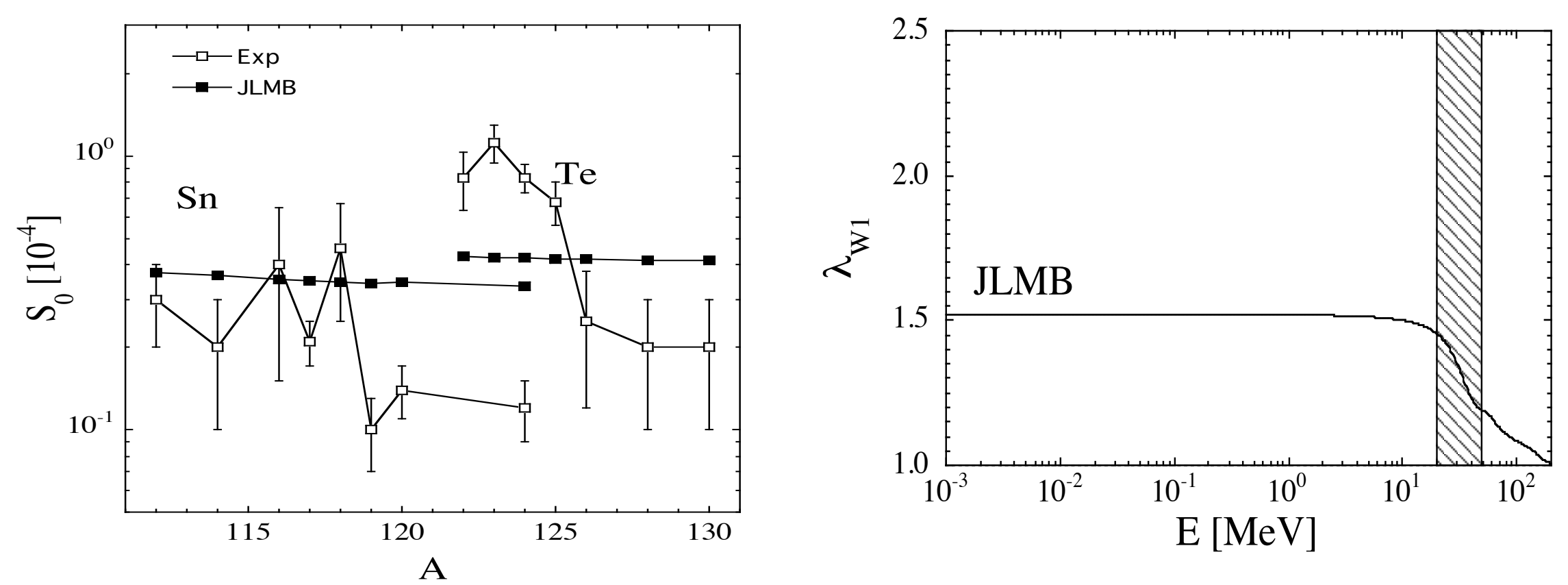
Some low-energy data can be used to constrain the isovector imaginary potential:

\section{the neutron strength function $S_{0}$ and $S_{1}$}

Re-renormalization of the isovector imaginary potential on $S$-wave neutron strength data
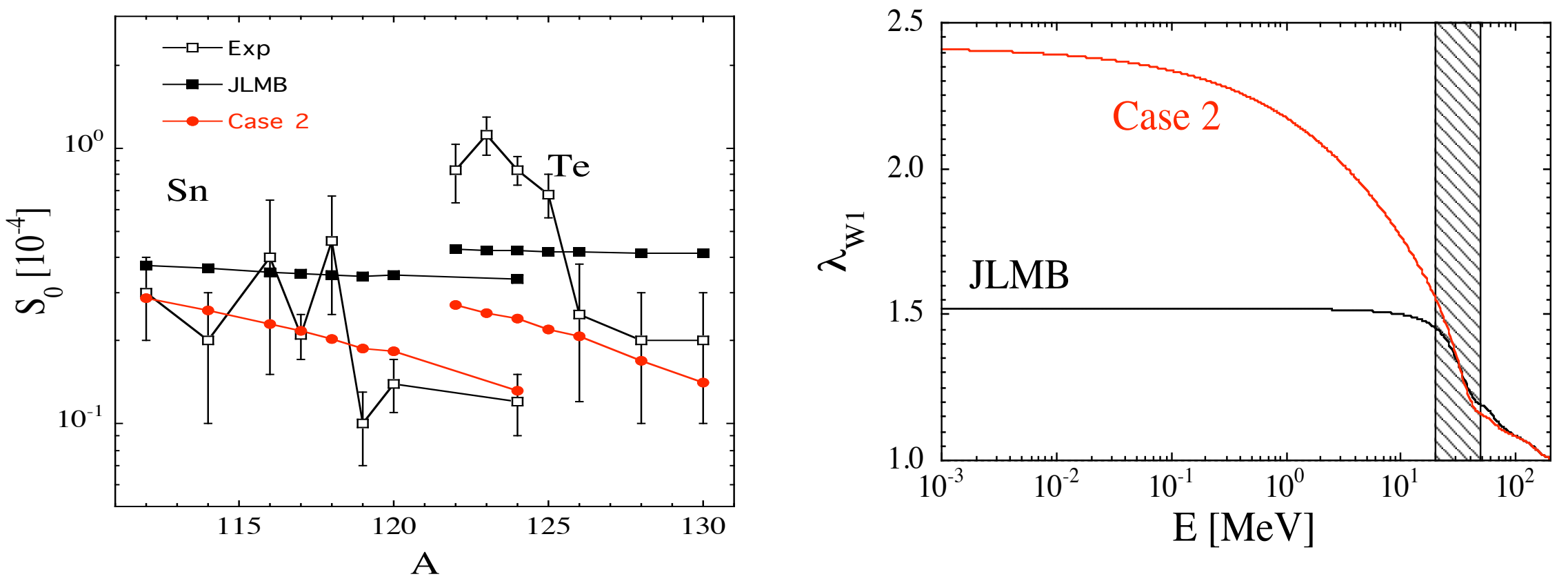
Some low-energy data can be used to constrain the isovector imaginary potential:

the neutron strength function $S_{0}$ and $S_{1}$

Re-renormalization of the isovector imaginary potential on $S$-wave neutron strength data

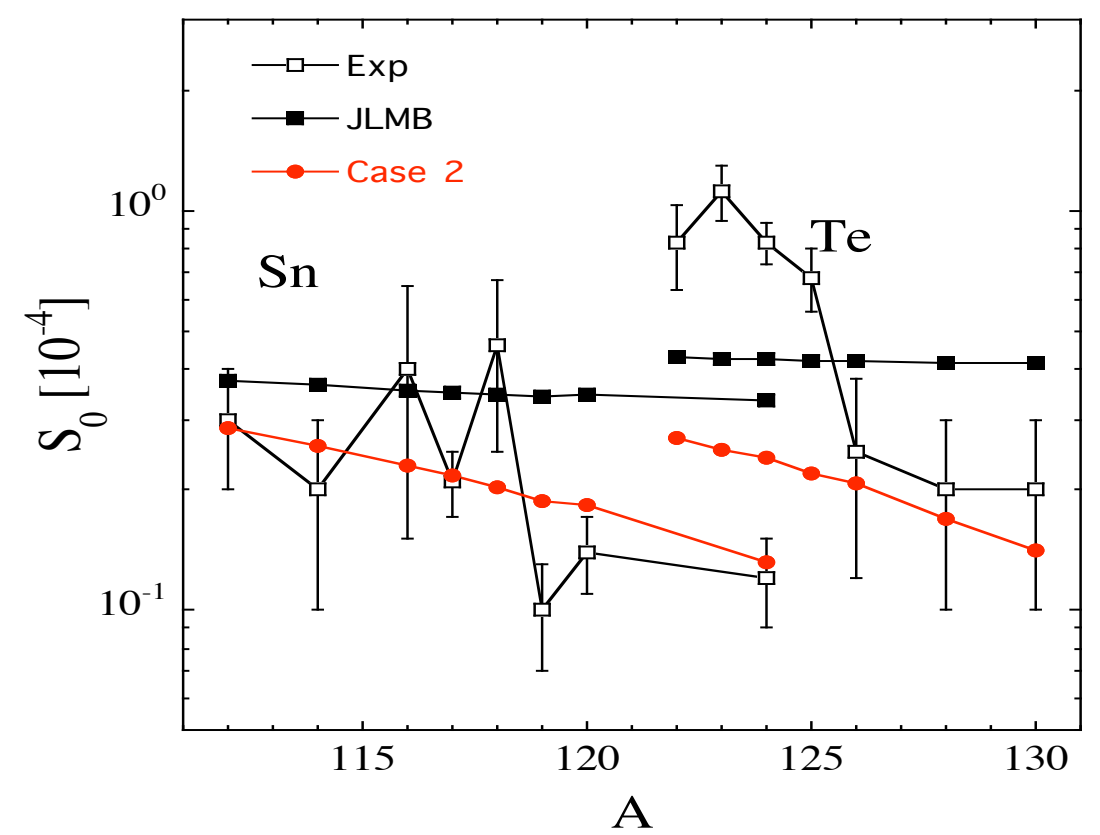

Impact on radiative neutron capture:

large reduction of the radiative capture by exotic n-rich nuclei

--> very sensitive to the isospin dependence of the S-wave neutron strength
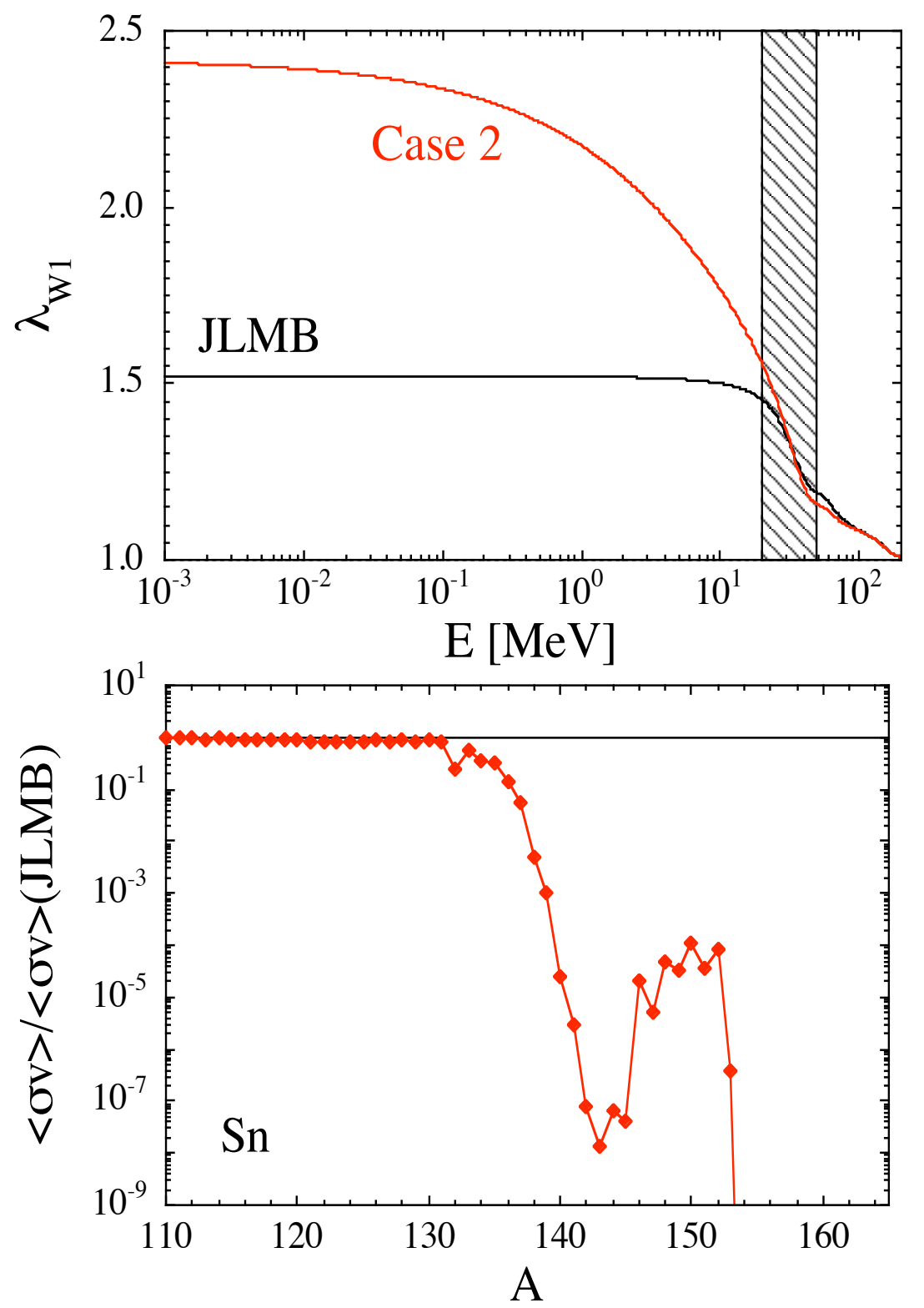
Drastic impact on the resonant capture by exotic neutron-rich nuclei

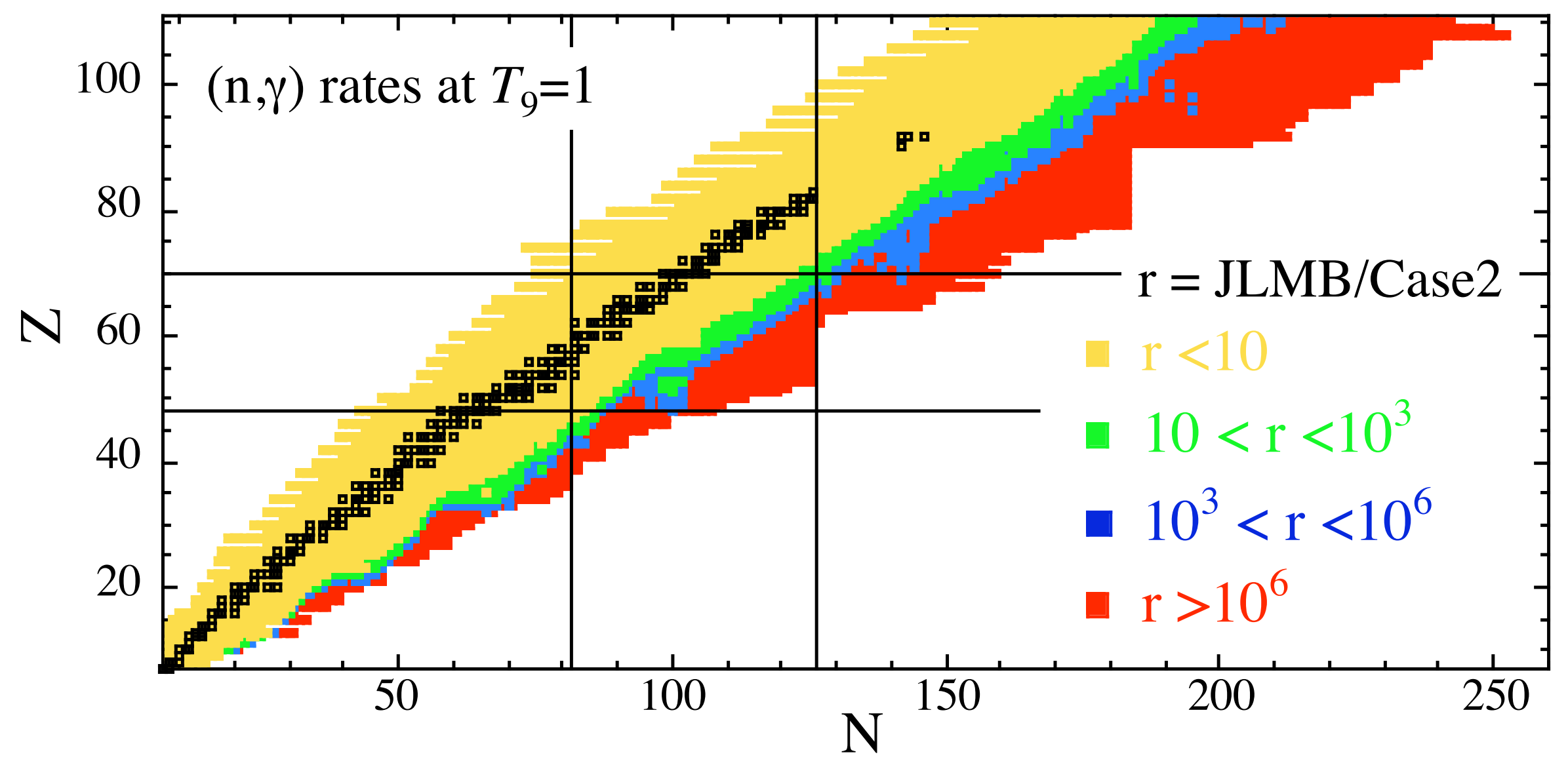

In that case, n-capture by exotic nuclei is dominated by the direct capture mechanism --> all calculations (nuclear \& astro) need to be revisited. 
TALYS predictions of the radiative neutron capture rates

Reaction rates at $\mathrm{T}_{8}=3.5(\mathrm{kT} \sim 30 \mathrm{keV})$

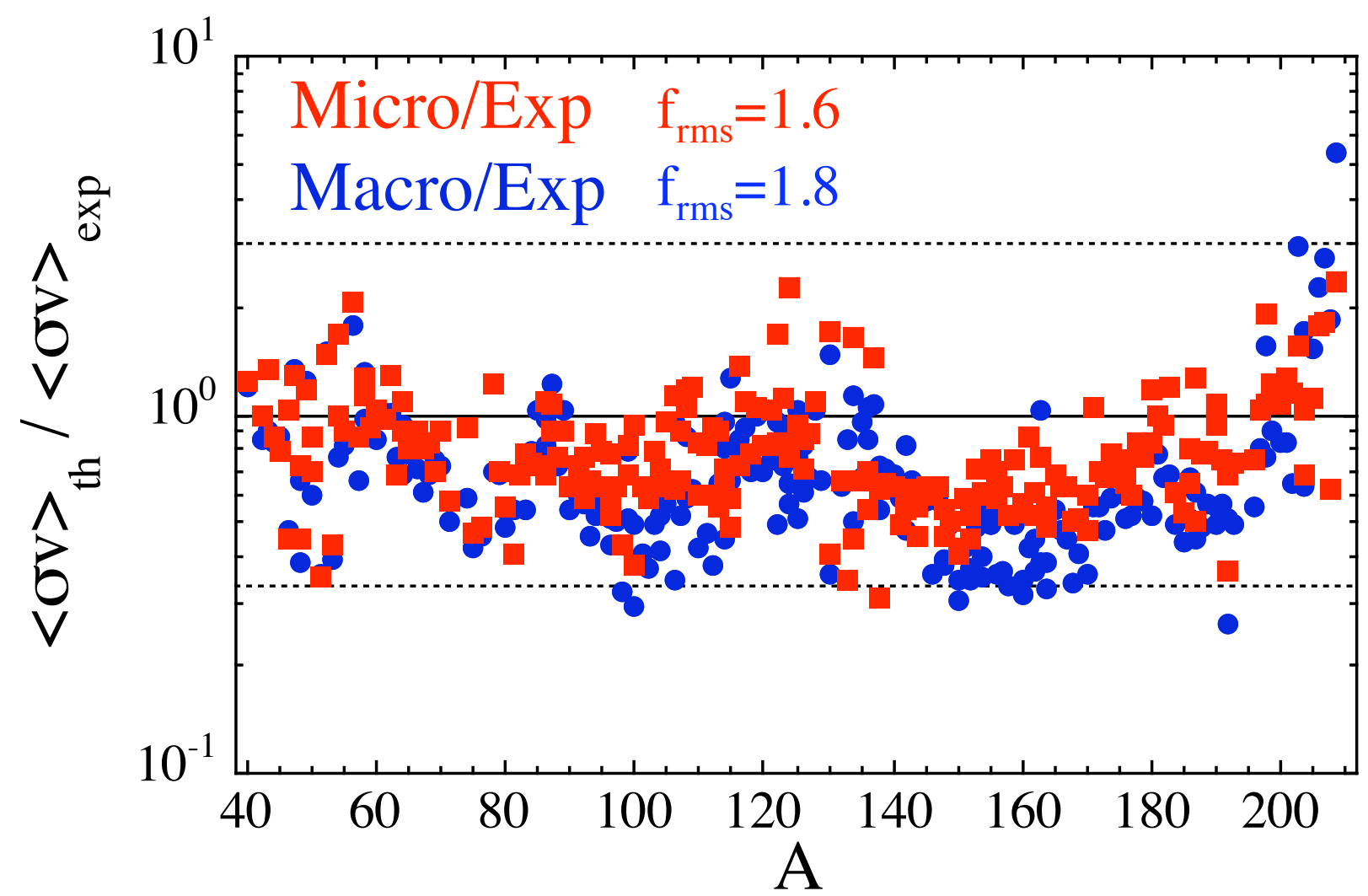

Ground -state properties: Nuclear Level Densities: E1-strength functions: Nucleon-Nucleus OMP:
«MACROSCOPIC » FRDM (Moller et al 95) BSFG (RIPL-2)

Lorentzian (Kopecky-Uhl 1990) WS-type (Koning et al., 2003)
«MICROSCOPIC » HFB-14 (S.G. et al. 2007) HFB Combinatorial (Hilaire et al, 2008) HFBCS+ QRPA (S.G. \& Khan 2003) WS-type (Koning et al., 2003) 
Uncertainties in the prediction of the radiative neutron capture rate

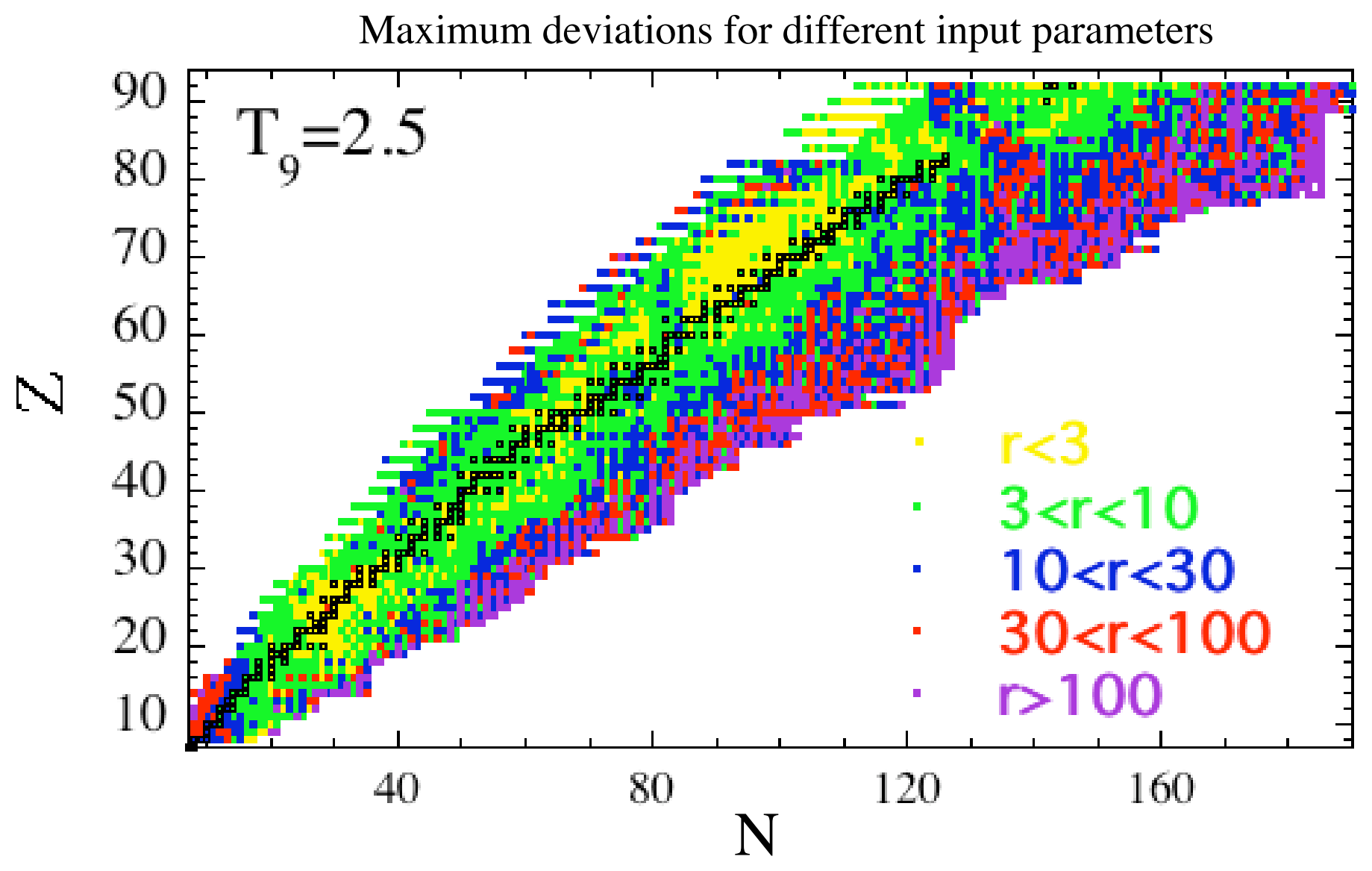

But still much larger deviation if use is made of specific nuclear ingredients (e.g JLMB) 
Uncertainties in the prediction of the radiative proton capture rate

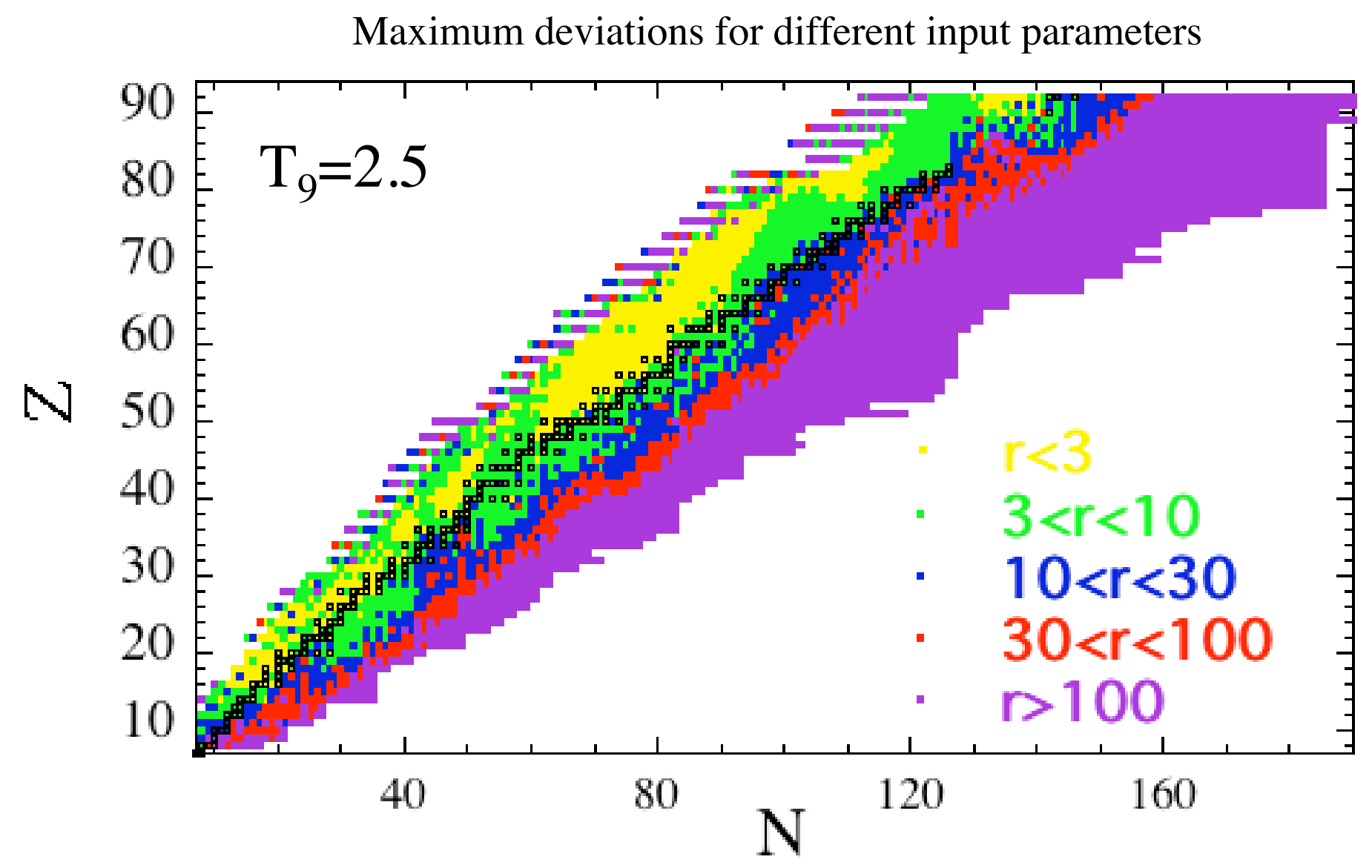


Uncertainties in the prediction of the radiative $\alpha$-capture rate

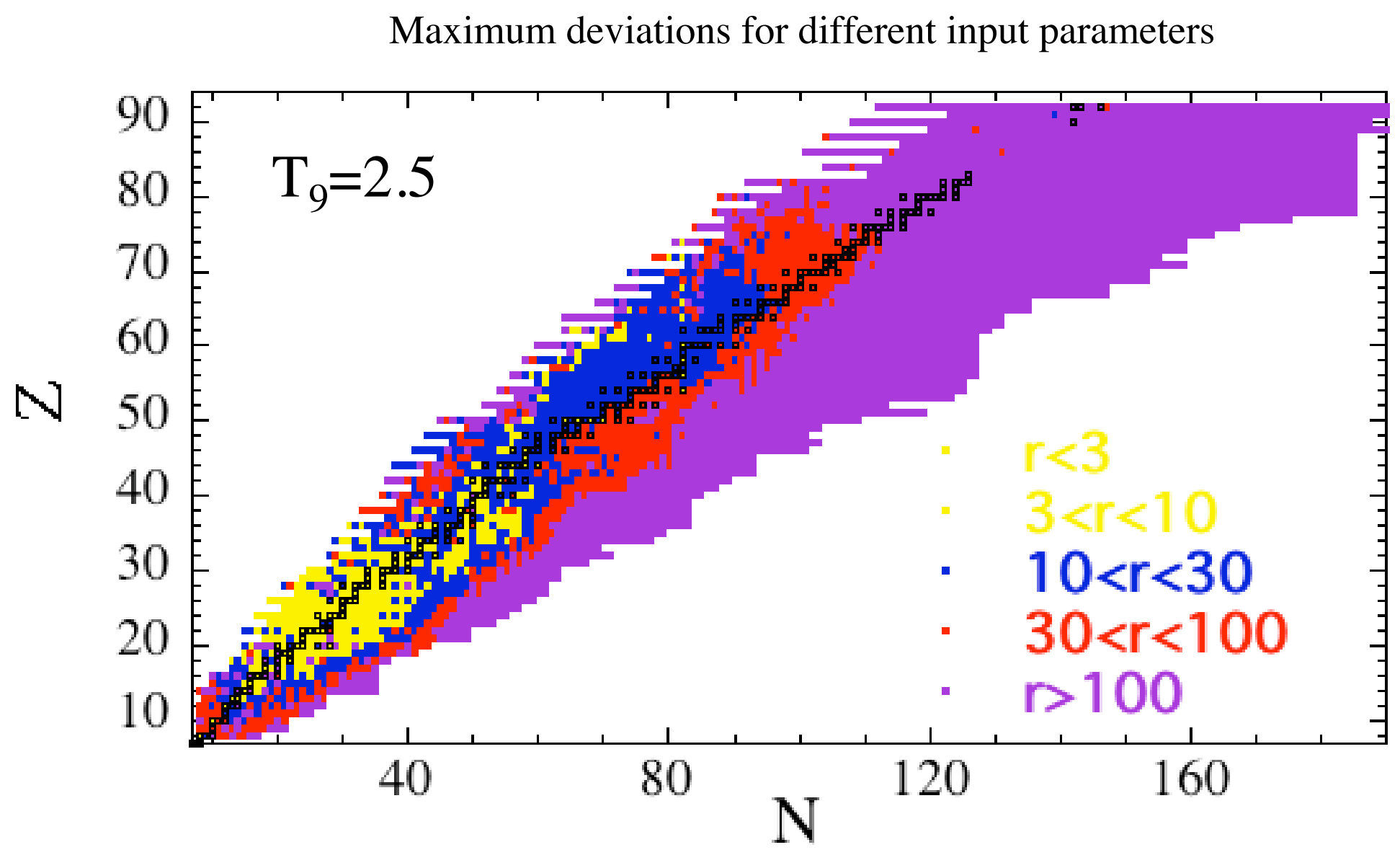




\section{Fission}




\section{Global models of fission barriers}

- Macroscopic-Microscopic Approaches

LDM model (Howard \& Moller 1980)

TF + FRDM shell corr. (Myers \& Swiatecki, 1996)

FRLDM (Moller, 2008)

- Approximation to Microscopic models

ETFSI model (Rayet, Pearson et al. 1995)

- Mean Field Model

HF-BCS model

HFB model 


\section{Nuclear Fission}

A charged liquid drop will only be stable against small distortions if the decrease in the Coulomb energy is smaller than the increase in surface energy.

$$
B(Z, A)=a_{V} A-a_{S} A^{2 / 3}-a_{\text {coul }} Z^{2} A^{-1 / 3}-a_{\text {sym }}\left(\frac{N-Z}{A}\right)^{2} A+\delta
$$

The coulomb energy is proportional to $Z^{2} / R$ while the surface energy is proportional to $R^{2}$. To characterize fission within the liquid drop approach, the fissility parameter is introduced

$$
x=\frac{E_{\text {coul }}(\beta=0)}{2 E_{S}(\beta=0)}=\frac{a_{\text {coul }}}{2 a_{S}} \frac{Z^{2}}{A} \quad \begin{aligned}
& \text { if } x<1-->\text { stable against fission } \\
& \text { if } x>1-->\text { unstable against fission }
\end{aligned}
$$

The liquid drop model only predicts one barrier. Shell effects give rise to the double-humped picture

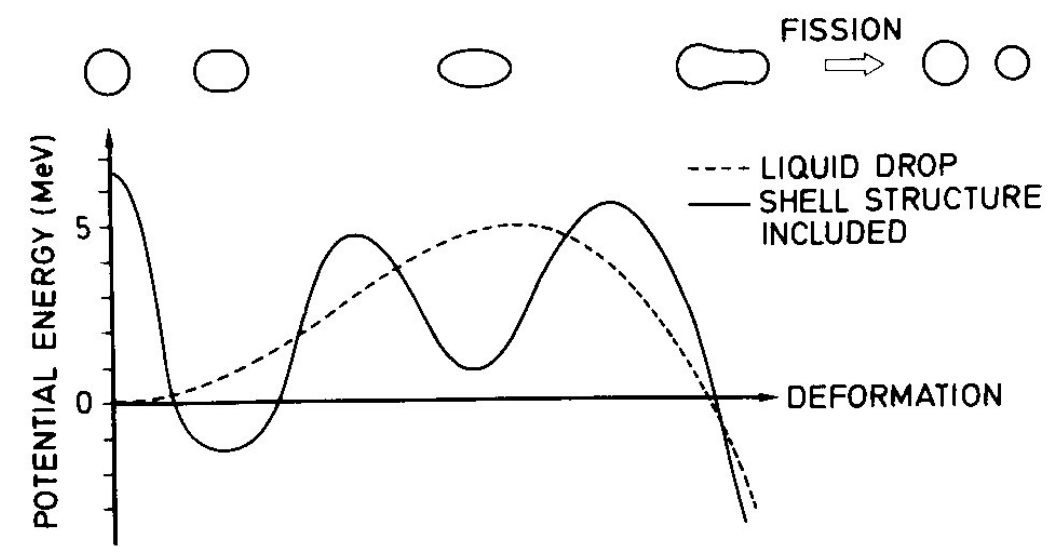

The same methods as those developed to predict mass formulas can be used to estimate fission barriers in the deformation plane. Macroscopic as well microscopic approaches have been developed. 
The major difficulty is the proper description of the nuclear deformation along the fission path.

The fission path, including the various maxima and minima along the most "suitable", i.e energetically favourable, (static) path from the equilibrium deformation to the scission point.

It remains a complex multi-dimensional problem. In addition, the fission process of interest is always a dynamical process, making the prediction of fission probability extremely difficult.
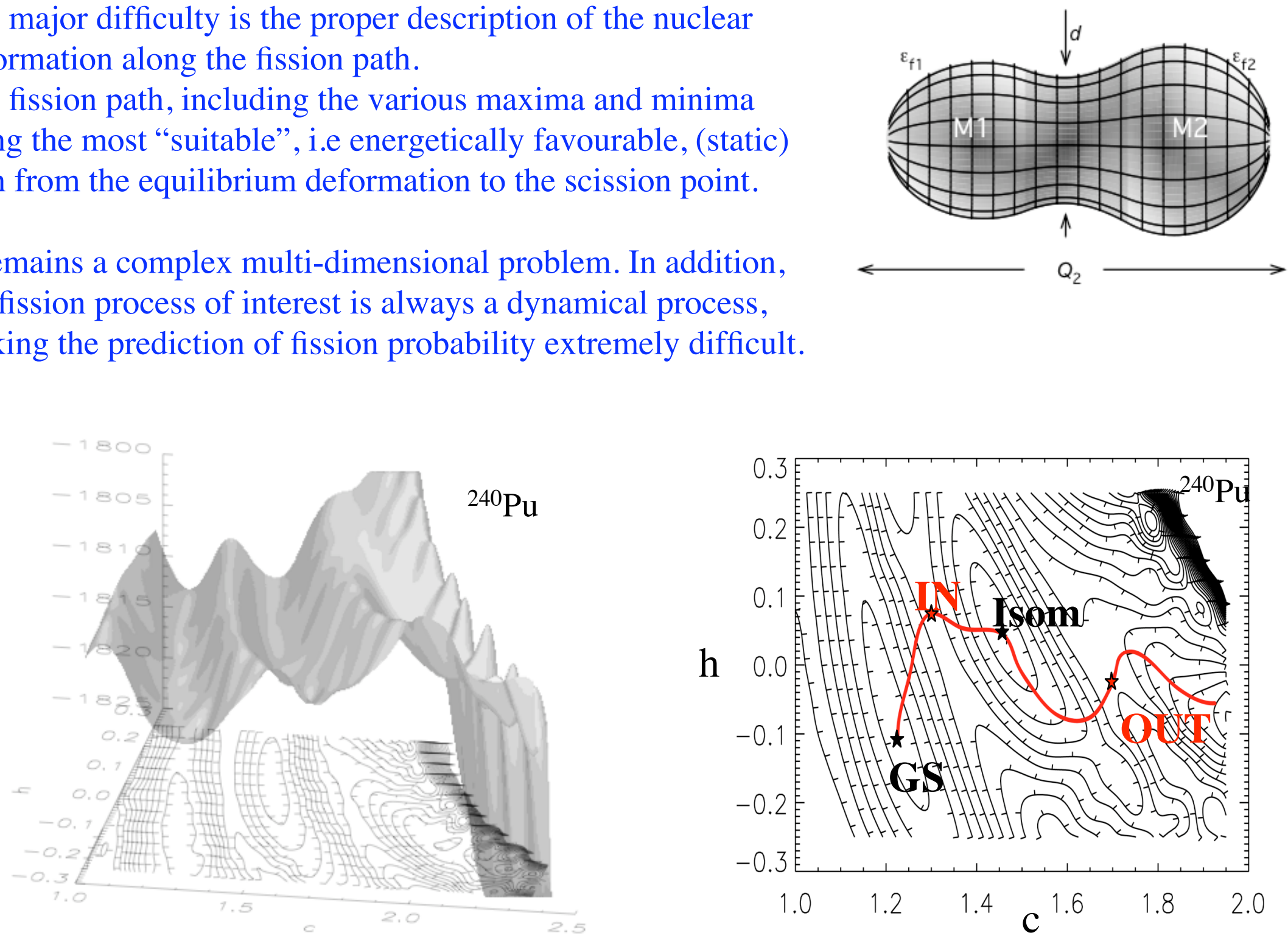


\section{Ingredients of relevance to estimate fission properties}

$$
T(E, J, \pi)=\int_{0}^{E} P(E-\varepsilon) \rho(\varepsilon, J, \pi) d \varepsilon\left\{\begin{array}{l}
P(E)=\frac{1}{1+\exp (2 K)} \\
K= \pm \int_{a}^{b}\left[2 \mu(E-V(\beta)) / \hbar^{2}\right]^{1 / 2} d \beta
\end{array}\right.
$$

Hill-Wheeler approximation: $\quad P^{H W}=\frac{1}{1+\exp \left[2 \pi\left(V_{0}-E\right) / \hbar \omega\right]}$

Fundamental ingredients:

$\left.\begin{array}{l}\text { - Fission barrier heights } \\ \text { - Fission barrier widths }\end{array}\right\}$ Fission path

- Nuclear Level Densities at saddle points

\section{MAJOR CHALLENGE: COHERENT PREDICTIONS OF ALL INPUTS}

Only experimentally-based systematics or phenomenological models are used Clear lack of sound models to predict the barrier height \& width and NLD for unknown nuclei 
Three fission modes play an important role in nucleosynthesis (essentially the r-process) applications:

- spontaneous fission: strongly dependent on the fission barrier height

- neutron-induced fission: strongly dependent on $S_{n}-B_{f}$ (for neutron kinetic energy of $\sim \mathrm{keV} \sim k T$ )

- $\beta$-delayed fission, i.e fission following a $\beta$-decay: strongly dependent of $Q_{\beta}-B_{f}$ and $Q_{\beta}-B_{f}-S_{n}$

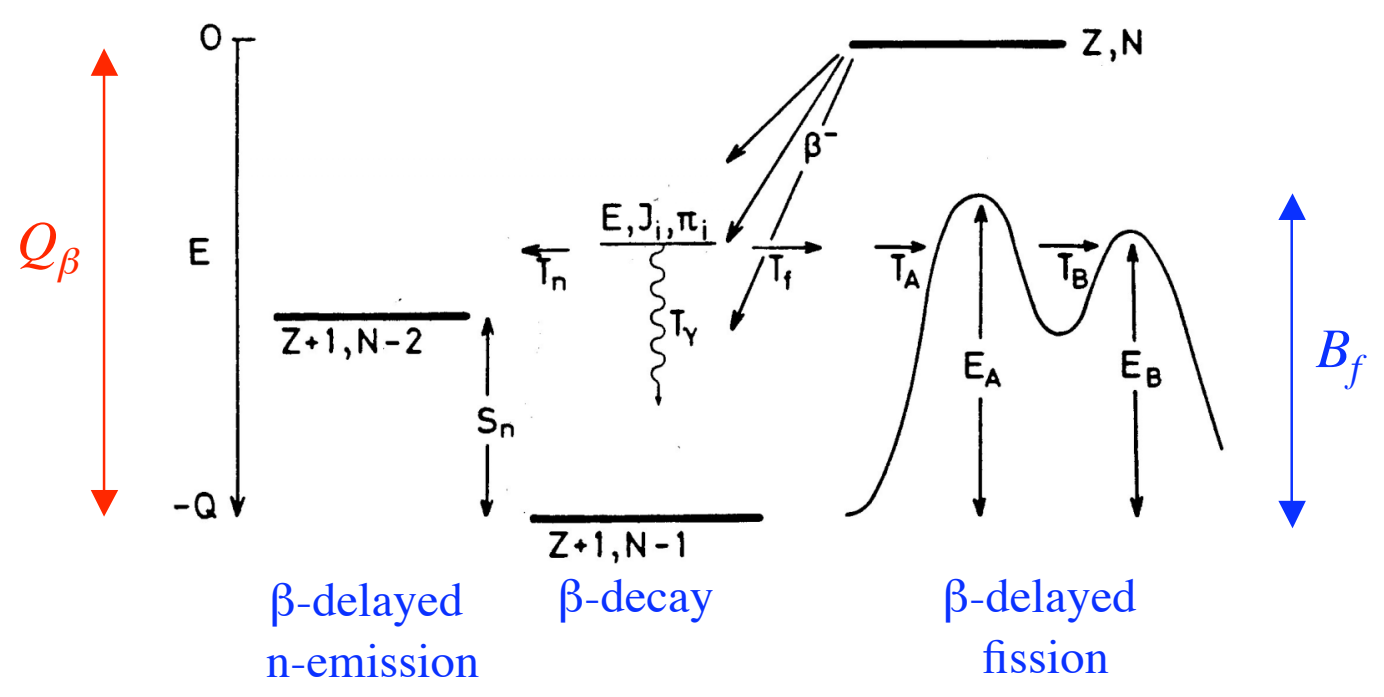

If it is already difficult to estimate reliably and accurately nuclear masses, and consequently neutron separation energies as well as $\beta$-decay $Q$-values, it remains extremely uncertain to estimate fission barriers (height, width, full path) for experimentally unknown nuclei. Only a few attempts have been made so fart to estimate fission properties of exotic nuclei, within the macroscopic-miroscopic (droplet-like model with shell and pairing corrections) as well as the mean-field Hartree-Fock (HF) method and one of its approximation known as the Extended Thomas-Fermi plus Strutinsky Integral (ETFSI). 


\section{ETFSI predictions of fission barriers}

Comparison of ETFSI predictions with "empirical" barriers

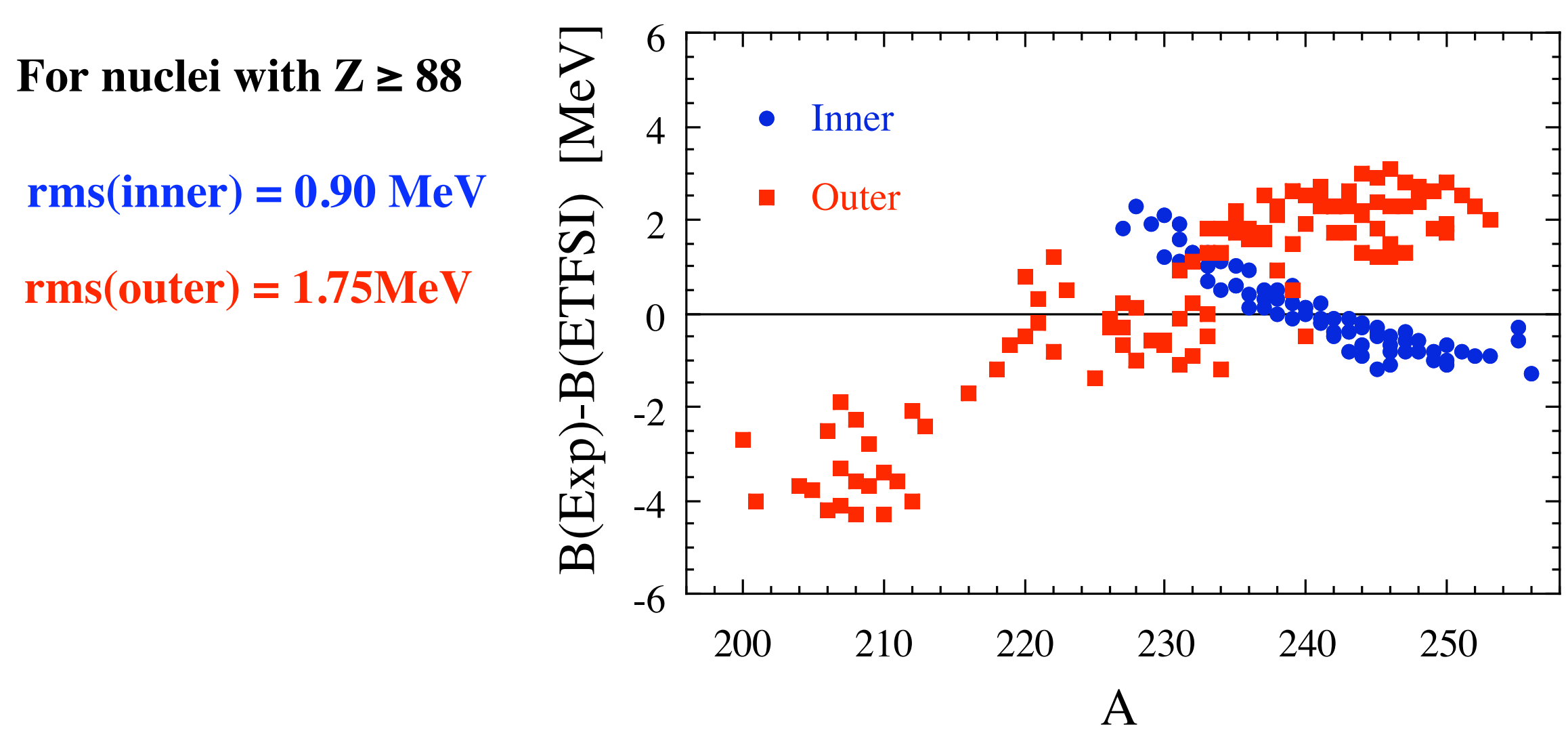




\section{FRLDM predictions of fission barriers}

Comparison of FRLDM predictions with "empirical" barriers

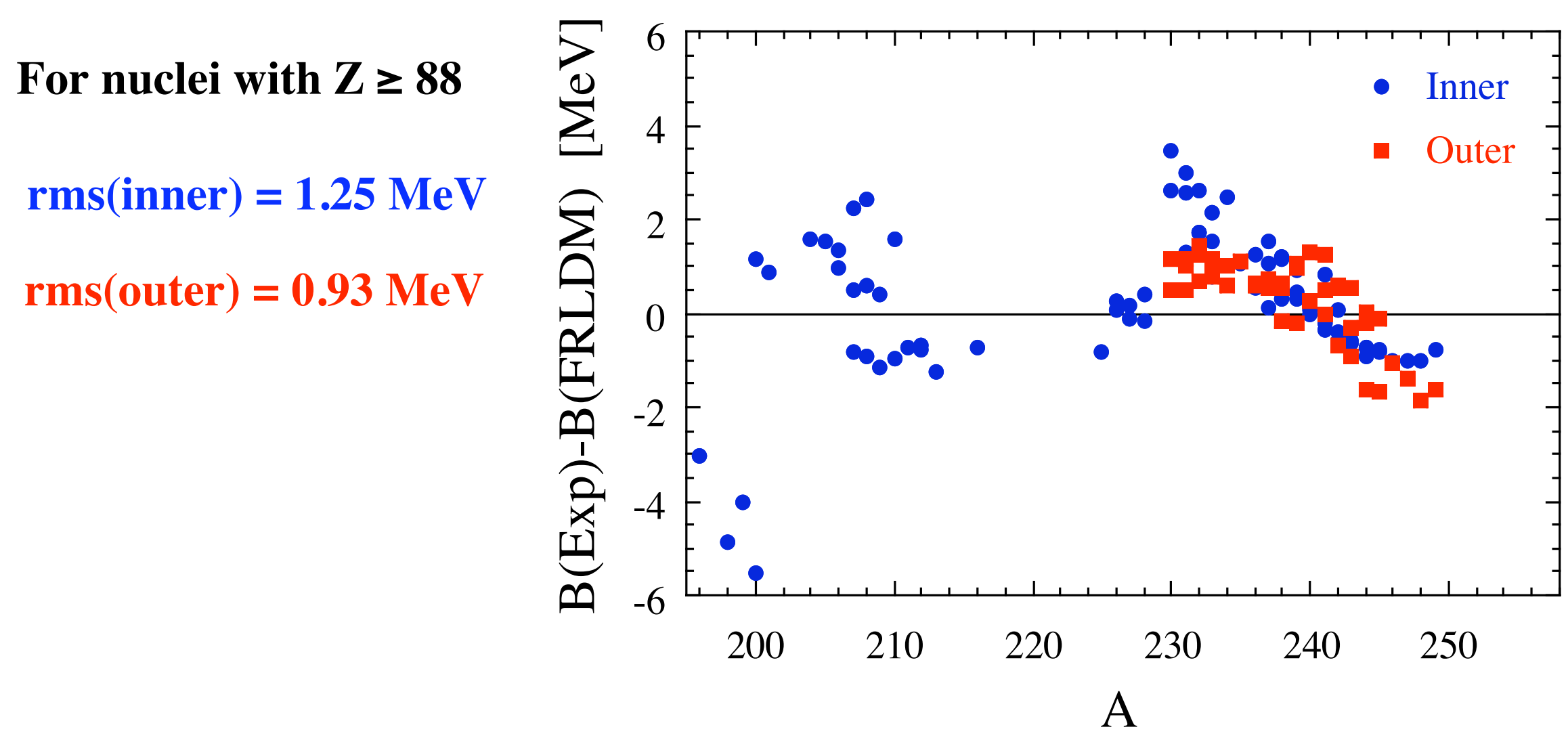




\section{HFB predictions of fission barriers}

Comparison of HFB-14 predictions with RIPL-2 "empirical” barriers

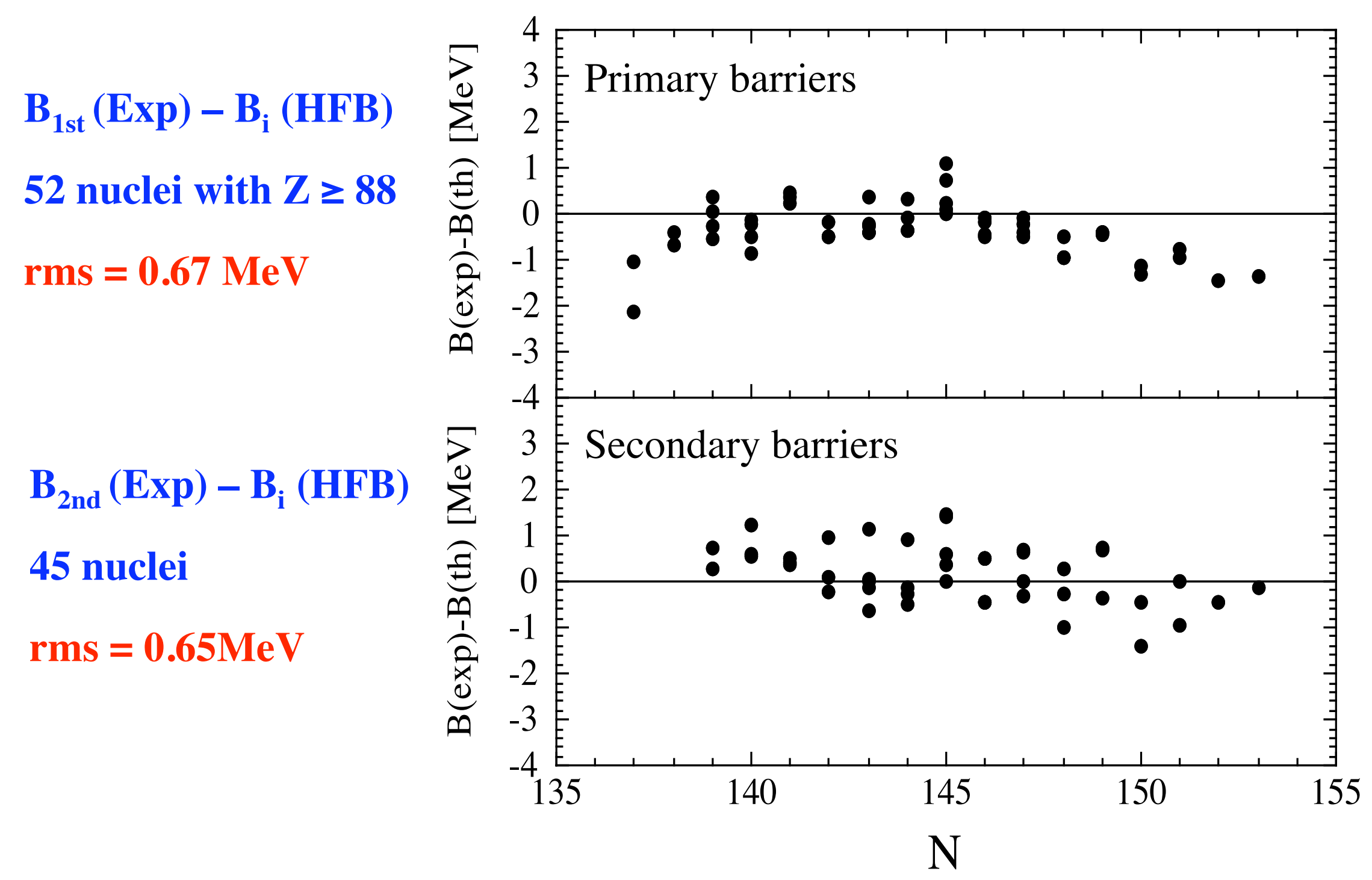


Comparaison of "empirical” (RIPL-3) primary barriers with model predictions

45 primary barriers $Z \geq 90$ from RIPL-3 compilation

$$
\begin{aligned}
& \operatorname{rms}(\text { HFB-14) }=0.60 \mathrm{MeV} \\
& \operatorname{rms}(\text { FRLDM })=0.81 \mathrm{MeV} \\
& \operatorname{rms}(\text { ETFSI })=0.57 \mathrm{MeV}
\end{aligned}
$$

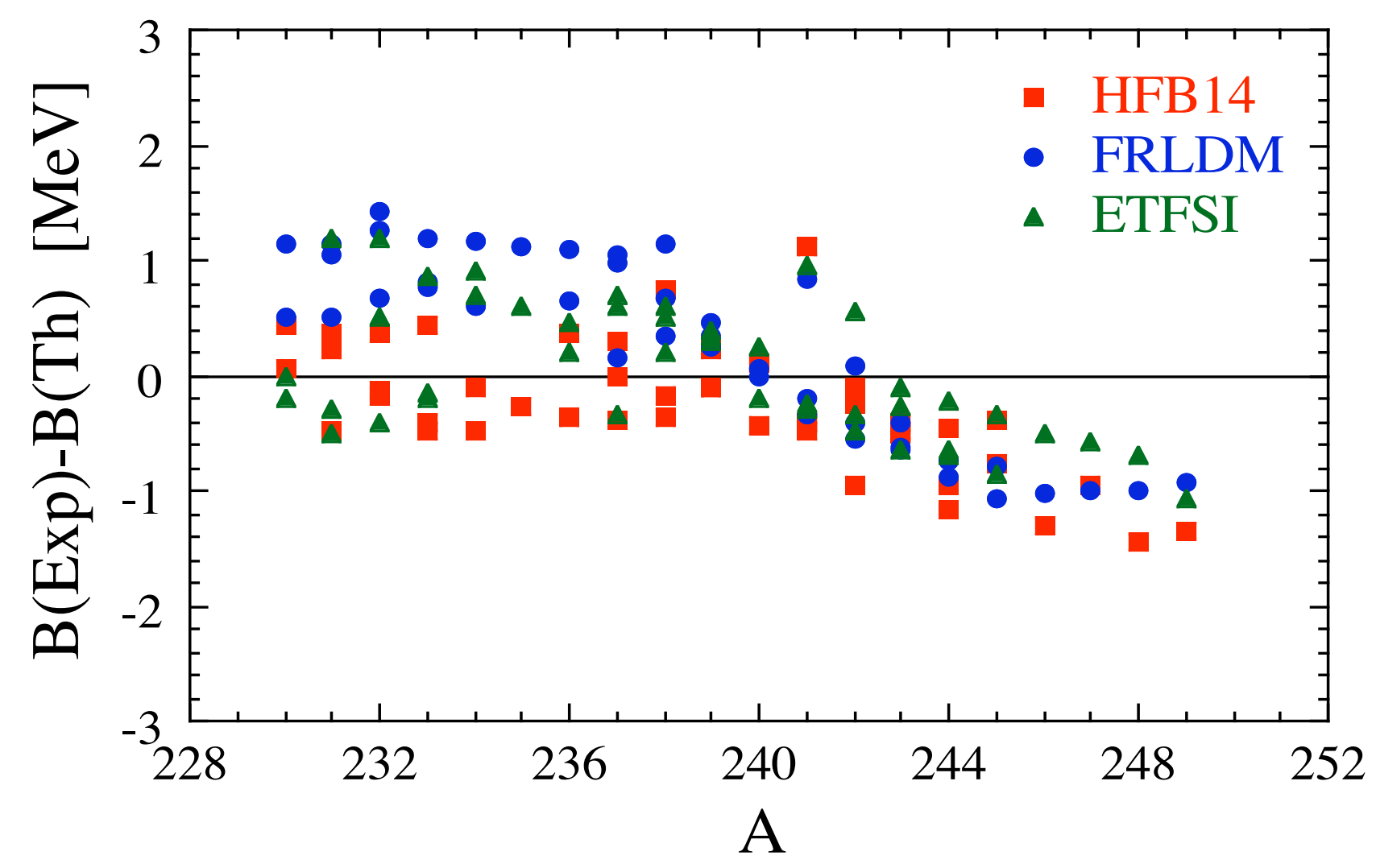




\section{Global predictions of fission barriers}

1000 HFB fission paths $(90 \leq \mathrm{Z} \leq 102)$ (publicly available at www-astro.ulb.ac.be)

(as well as coherently determined combinatorial NLD at saddle points)

\section{Close to $\beta$-stability}

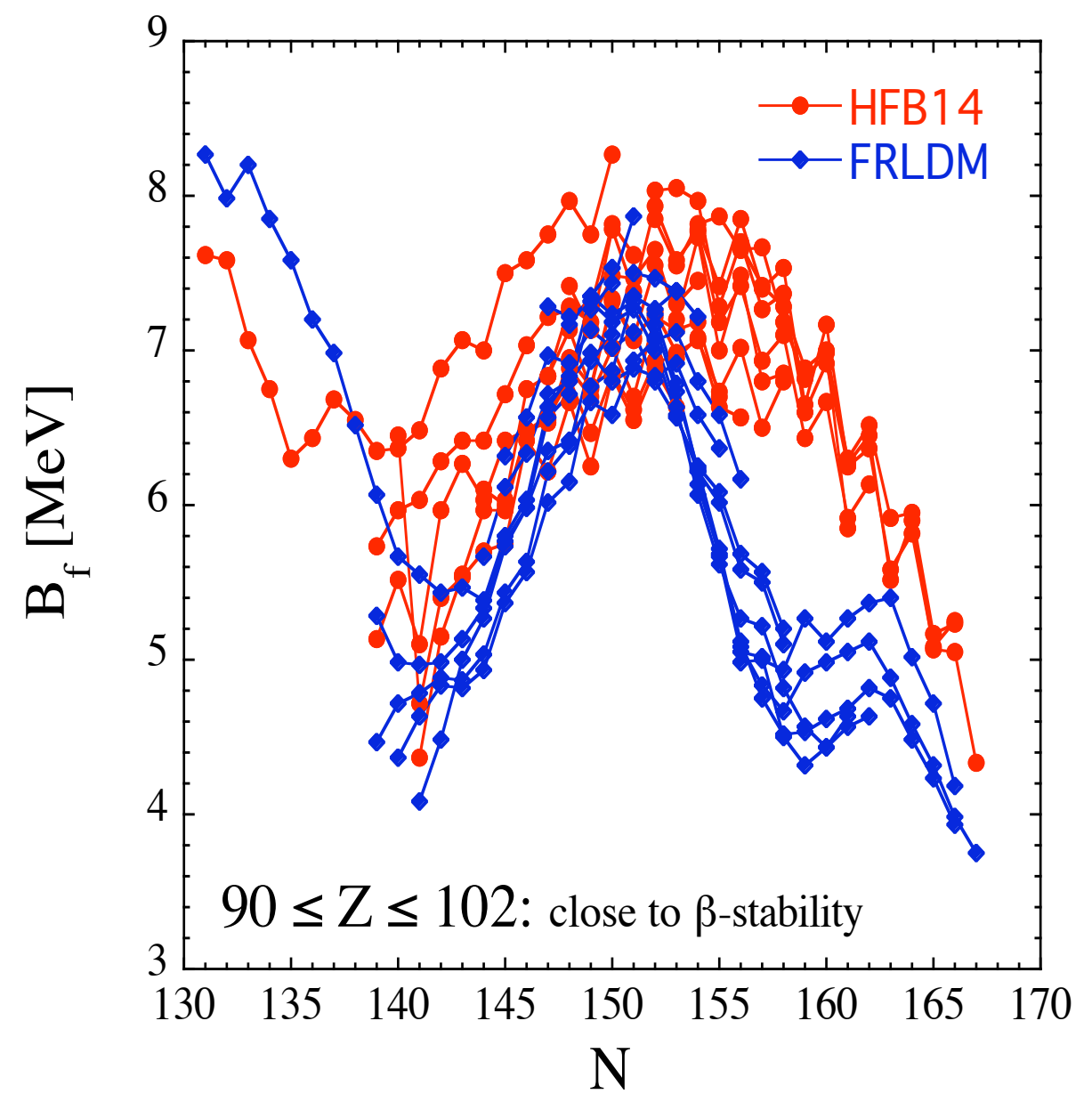

\section{Far away from $\beta$-stability}

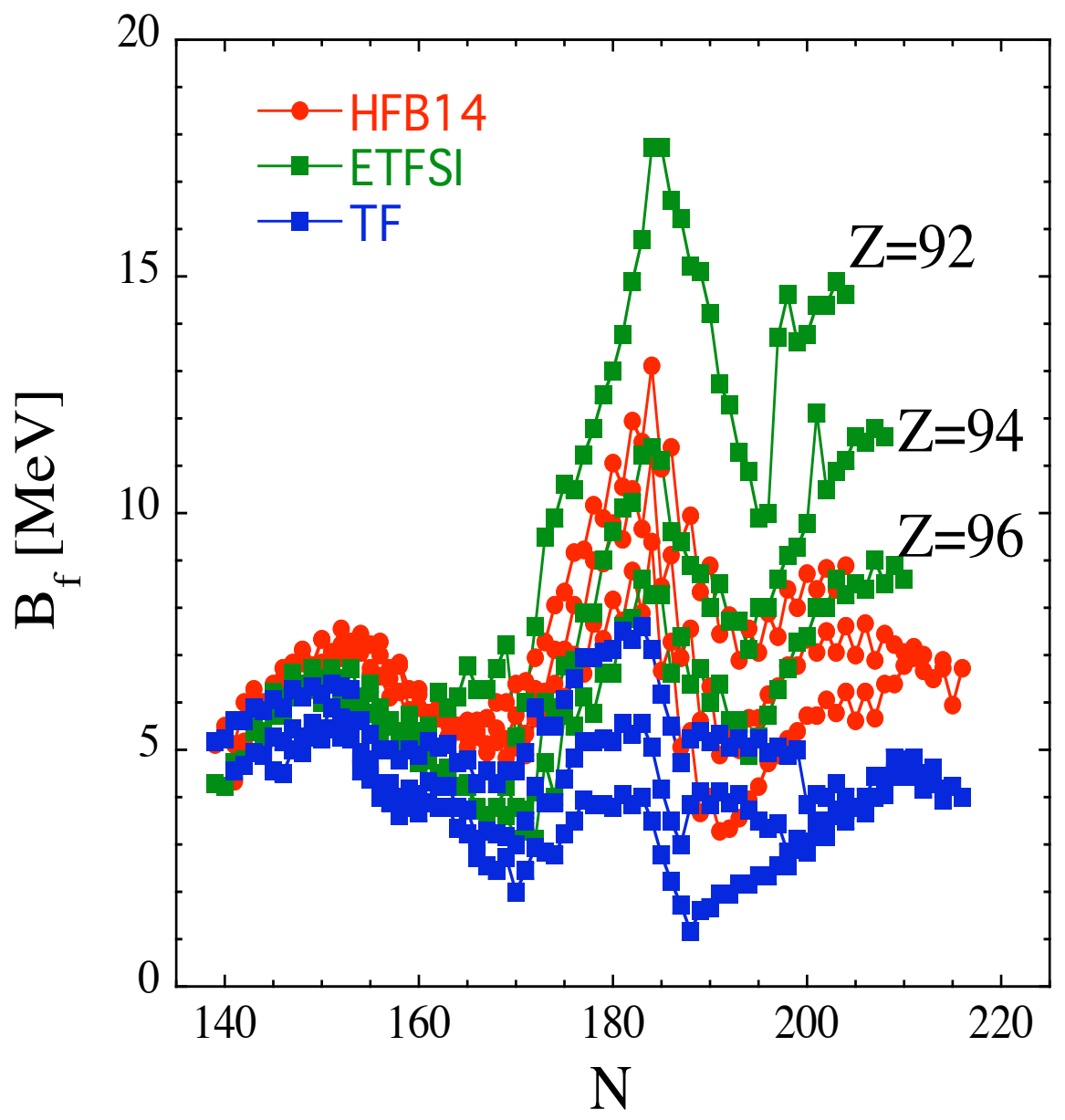


Extended Thomas-Fermi plus Strutinsky Integral (ETFSI) Fission Barriers

a compilation of 2300 microscopic $\mathrm{B}_{\mathrm{f}}: 78 \leq \mathrm{Z} \leq 120$

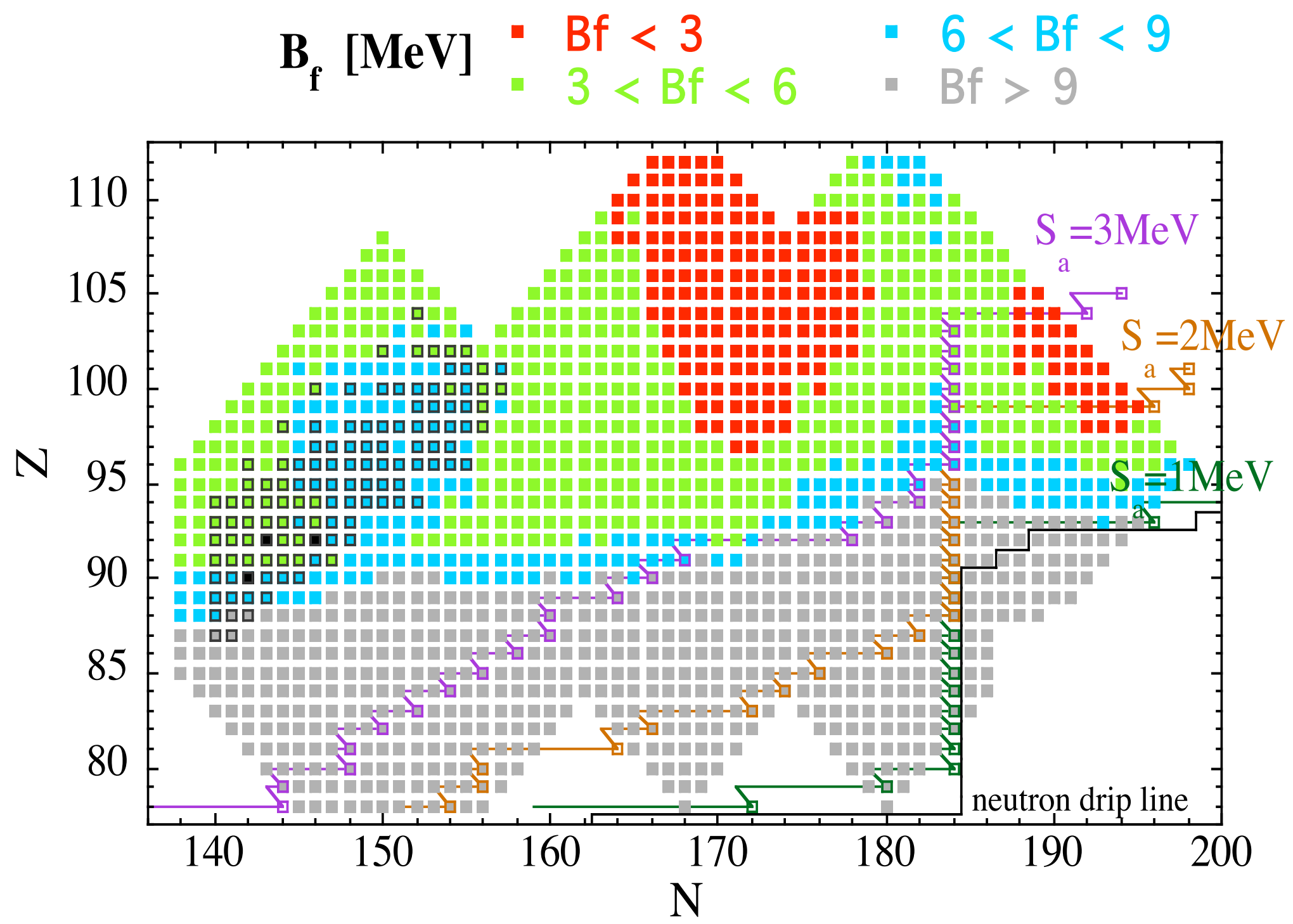


But calculations of fission probabilities require more than just barrier height !

Still no systematics for barrier width ....

(except from HFB-14 which also gives full fission path)

--> Usually assumed to be constant !?!

To what extent can the fission path be described by

a single- or a double-humped barrier (smoothly joined inverted parabolas)? 
HFB fission path: projection of the HFB-14 static path along the quadrupole deformation parameter $\beta_{2}$

$\mathrm{U}$ isotopes

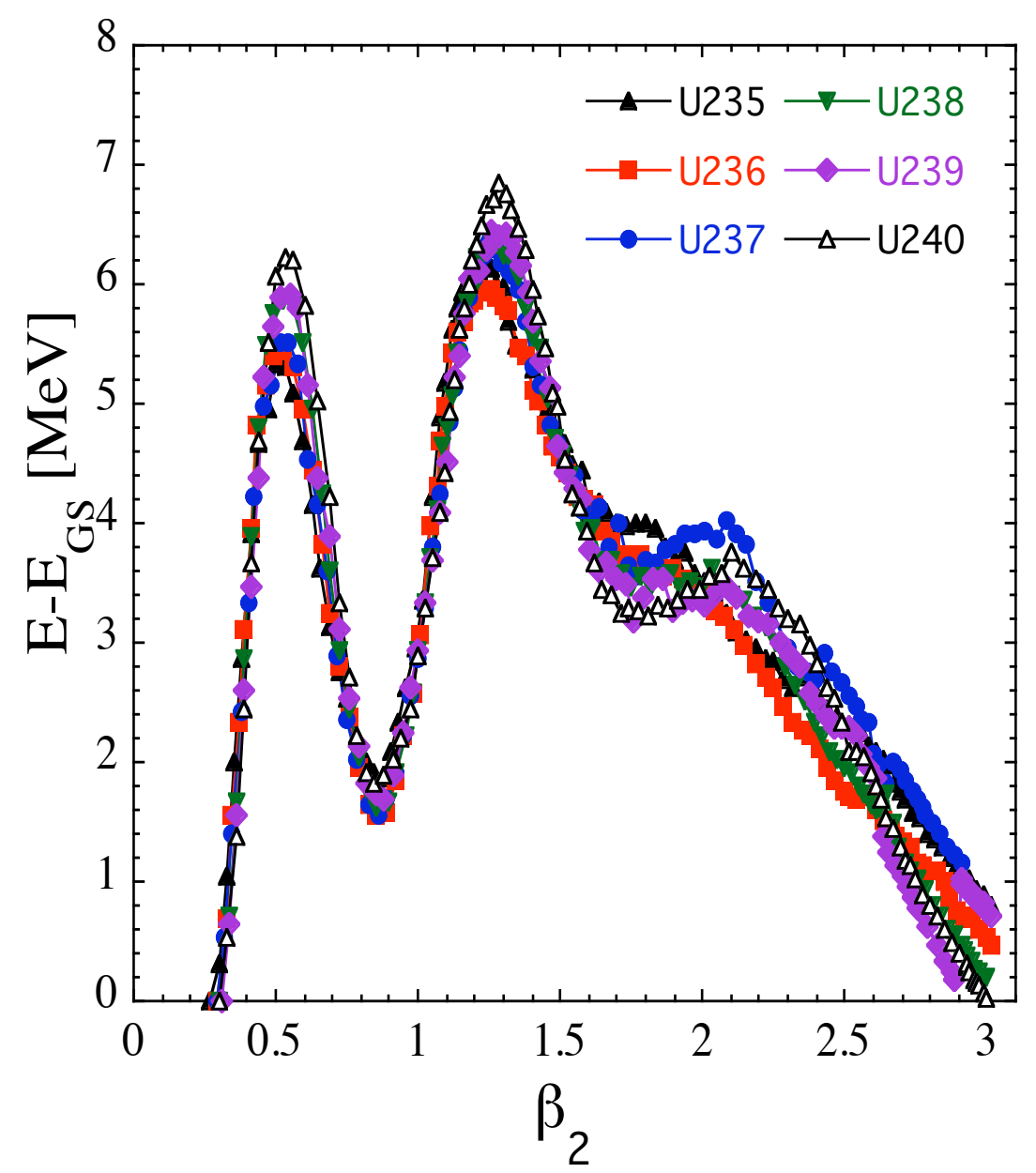

$\mathrm{Cm}$ isotopes

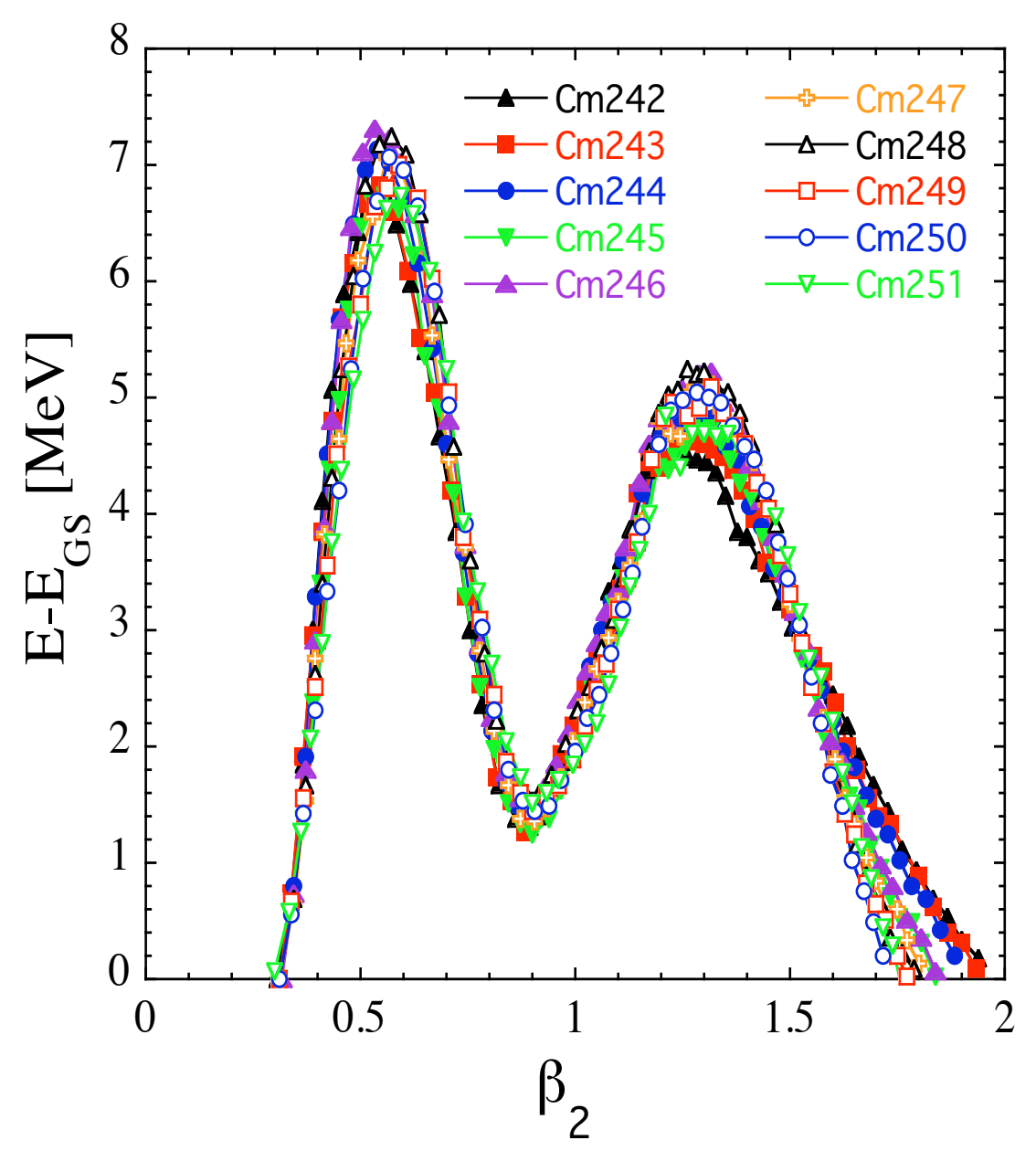




\section{The $\mathrm{Cm}$ isotopes in the n-rich region}

$$
252 \leq \mathrm{A} \leq 264
$$

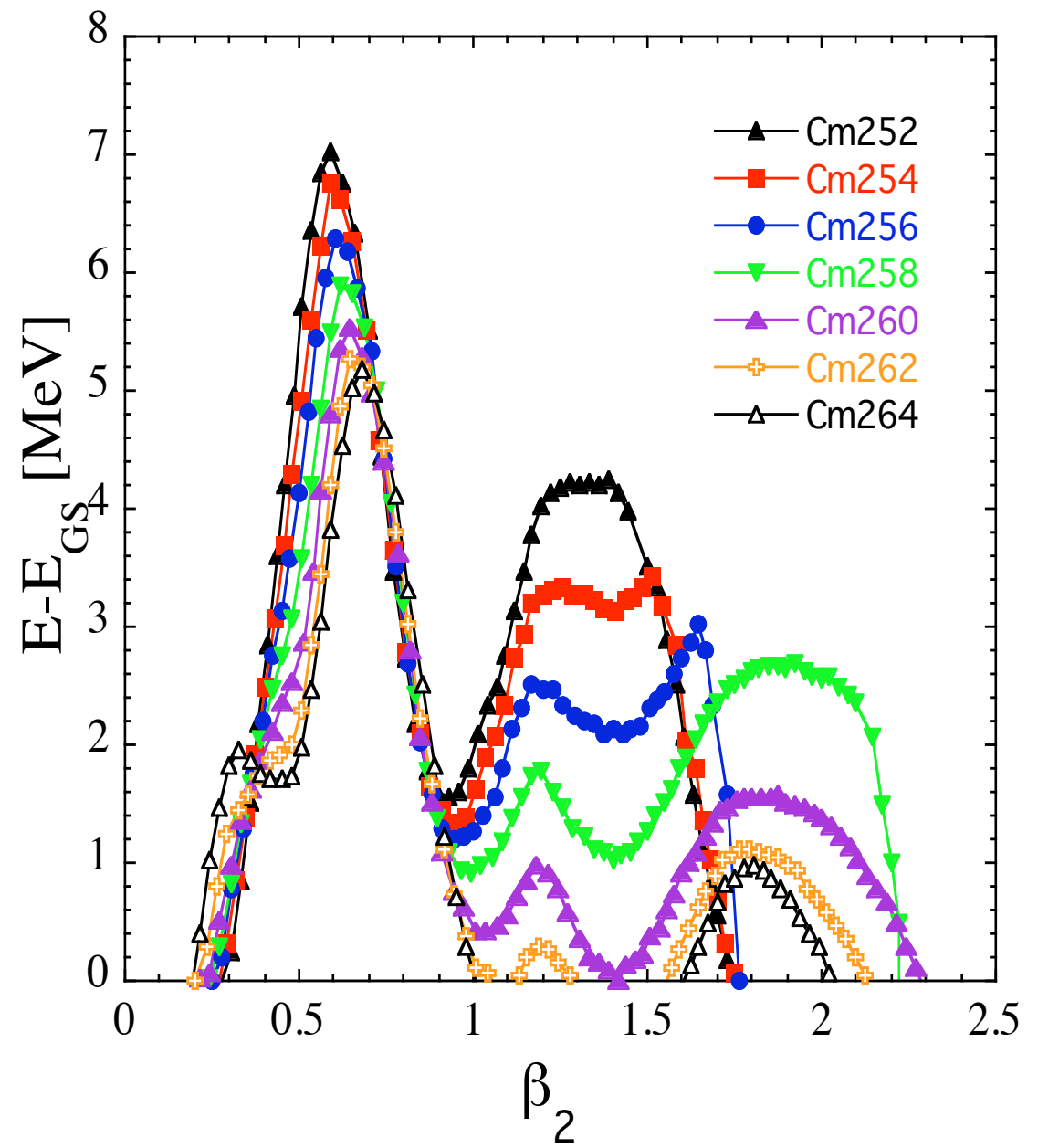

$$
270 \leq \mathrm{A} \leq 280
$$

${ }^{280} \mathrm{Cm}: \mathrm{N}=184$ shell closure

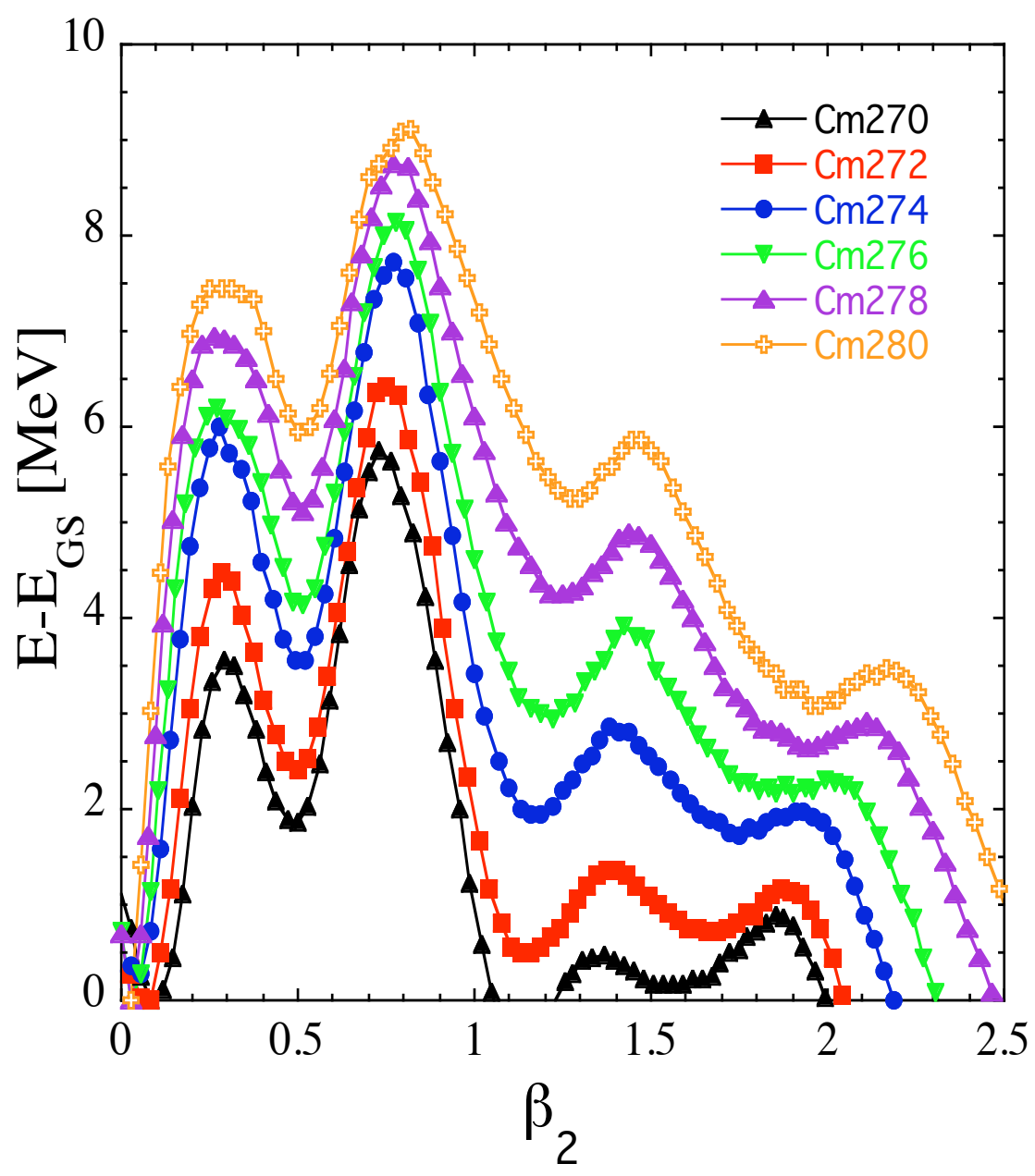


Determination of the barrier heigths and widths of the n-rich $\mathrm{Cm}$ isotopes
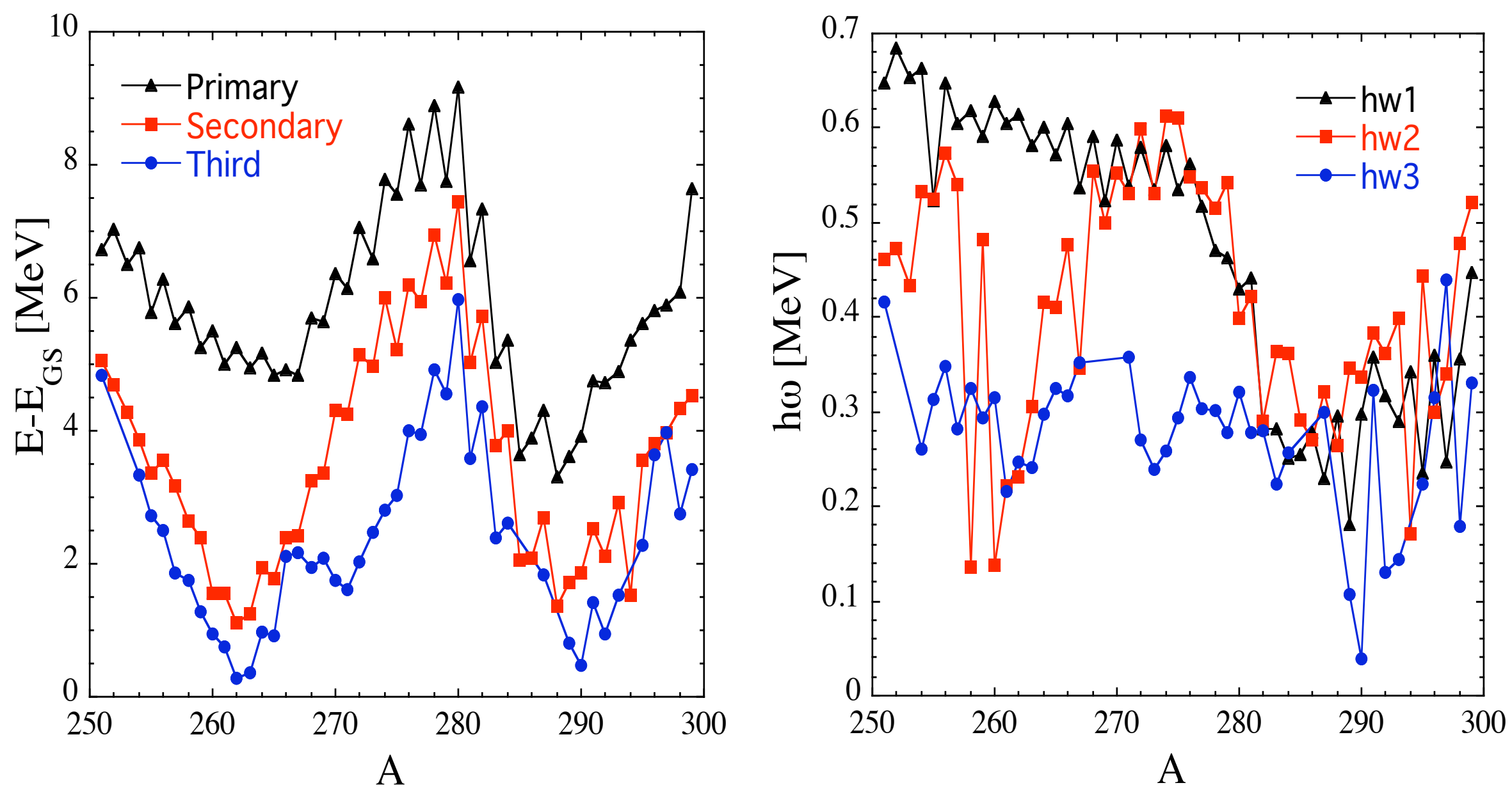
$(\mathrm{n}, \mathrm{f})$ reaction rates of $\mathrm{Pu}$ isotopes at $T=10^{9} \mathrm{~K}$

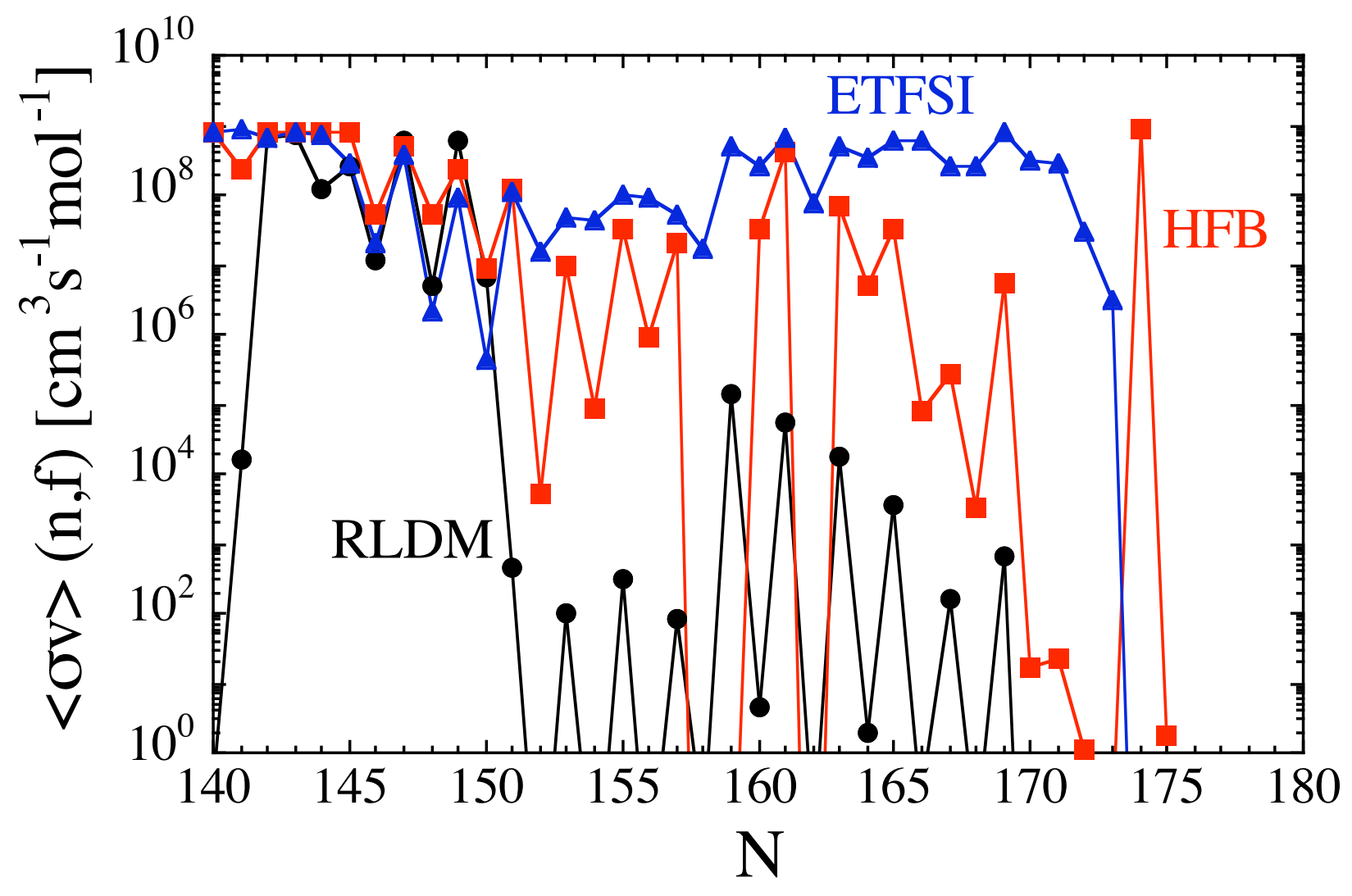

Still a lot of work required 


\section{Nuclear level densities at the saddle points}

HFB model constrained on Q,O,H moments provide at each deformation (and at saddle points) all nuclear properties needed to estimate the NLD

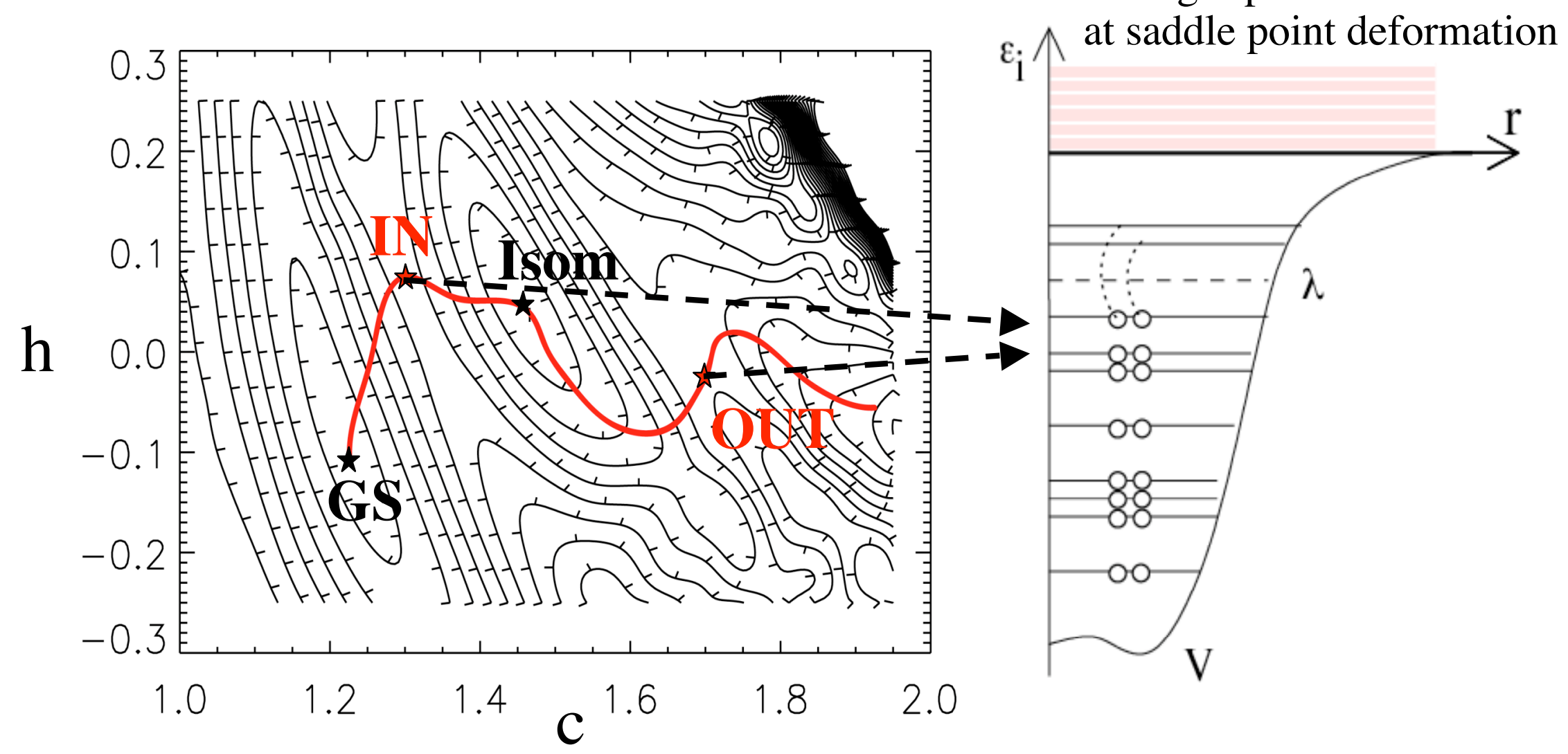

NLD traditionnally estimated from a highly-parametrized BSFG formula to reproducte $\sigma(\mathrm{n}, \mathrm{f})$ Possibility to estimate NLD at the saddle point within the HFB+Comb model 


\section{Nuclear Level Density at Saddle Points}

- Fission Barriers and saddle point deformations $(\mathrm{Q}, \mathrm{O}, \mathrm{H})$ determined within HFB method

- Nuclear properties (spl, pairing) at the inner and outer saddle points with constrained HFB model

- NLD in the framework of the microscopic combinatorial model based on HFB single-particle level and pairing predictions at the HFB saddle points

All ingredients described on the basis of the

same Skyrme effective interaction (BSk14) at GS and Saddle Points

$\longrightarrow$ NLD in a table format at inner and outer saddle points for about 1000 nuclei $(90 \leq \mathrm{Z} \leq 102)$

For inner barrier, usually predicted to be triaxial: $\quad \rho_{\text {triax }}=\sqrt{\frac{\pi}{2} \sigma_{\perp}} \times \rho_{\text {Comb }}$

For outer barrier, usually predicted to be left-right asymmetric: $\quad \rho_{\text {asym }}=2 \times \rho_{\text {Comb }}$ 
Prediction of the NLD at the fission saddle point and shape isomer
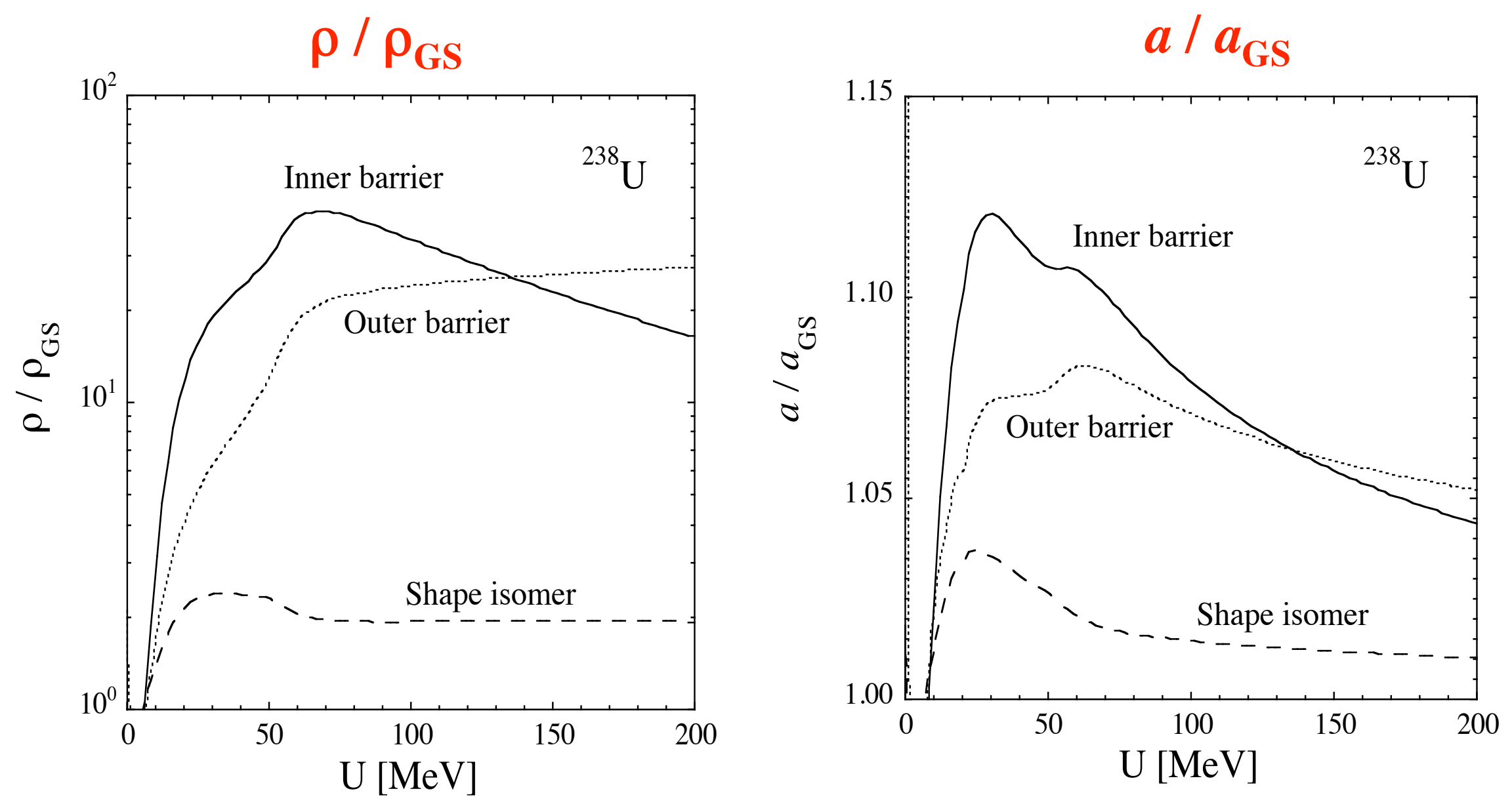

Equivalent $a$-parameter deduced from the Fermi-gas: $\rho(U)=\frac{\sqrt{\pi}}{12 a^{1 / 4} U^{5 / 4}} \mathrm{e}^{2 \sqrt{a U}}$ 
Prediction of $(n, f)$ cross sections with HFB fission paths \& HFB+Comb NLD
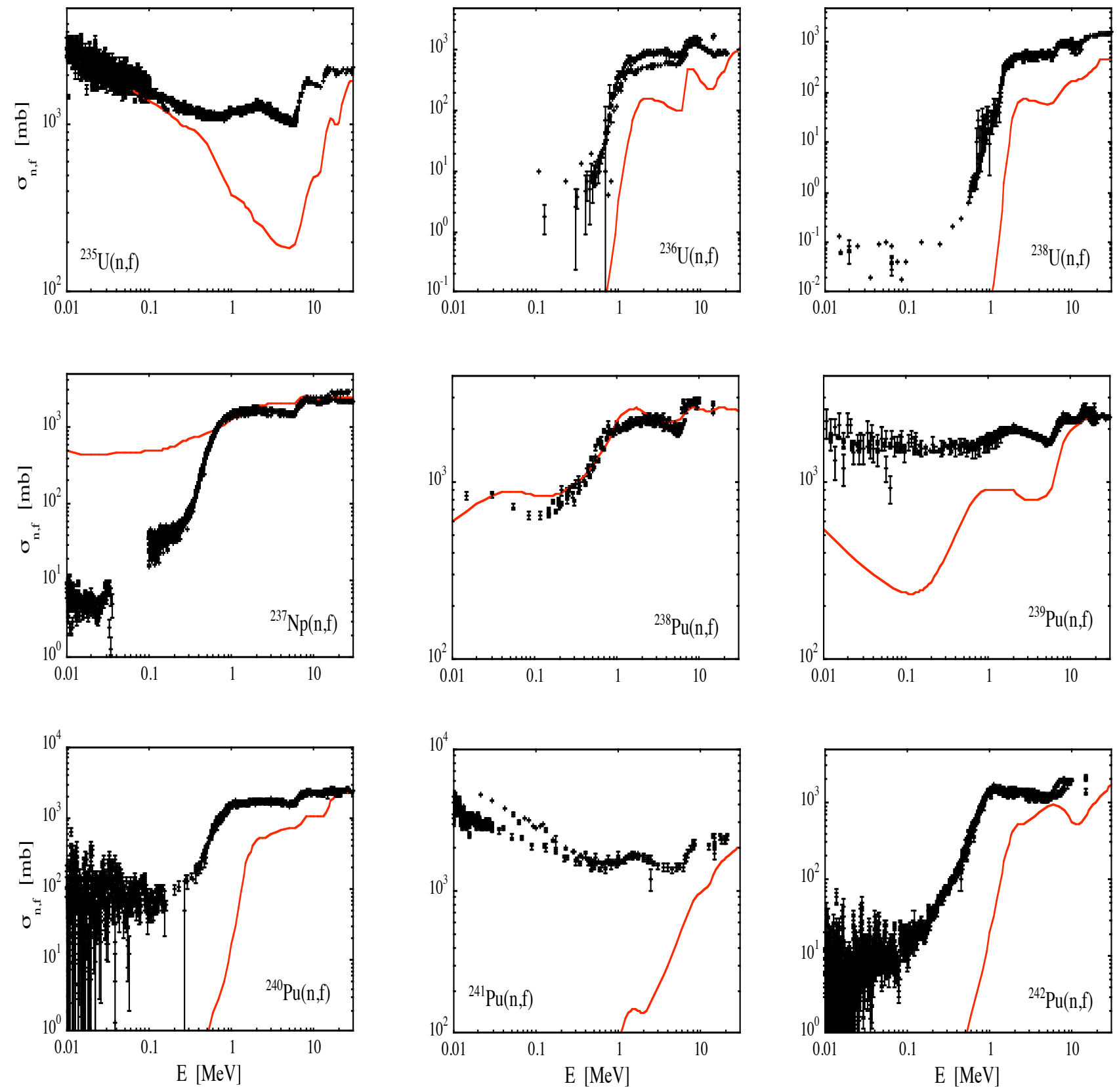
With renormalized HFB fission paths (individual or systematics)
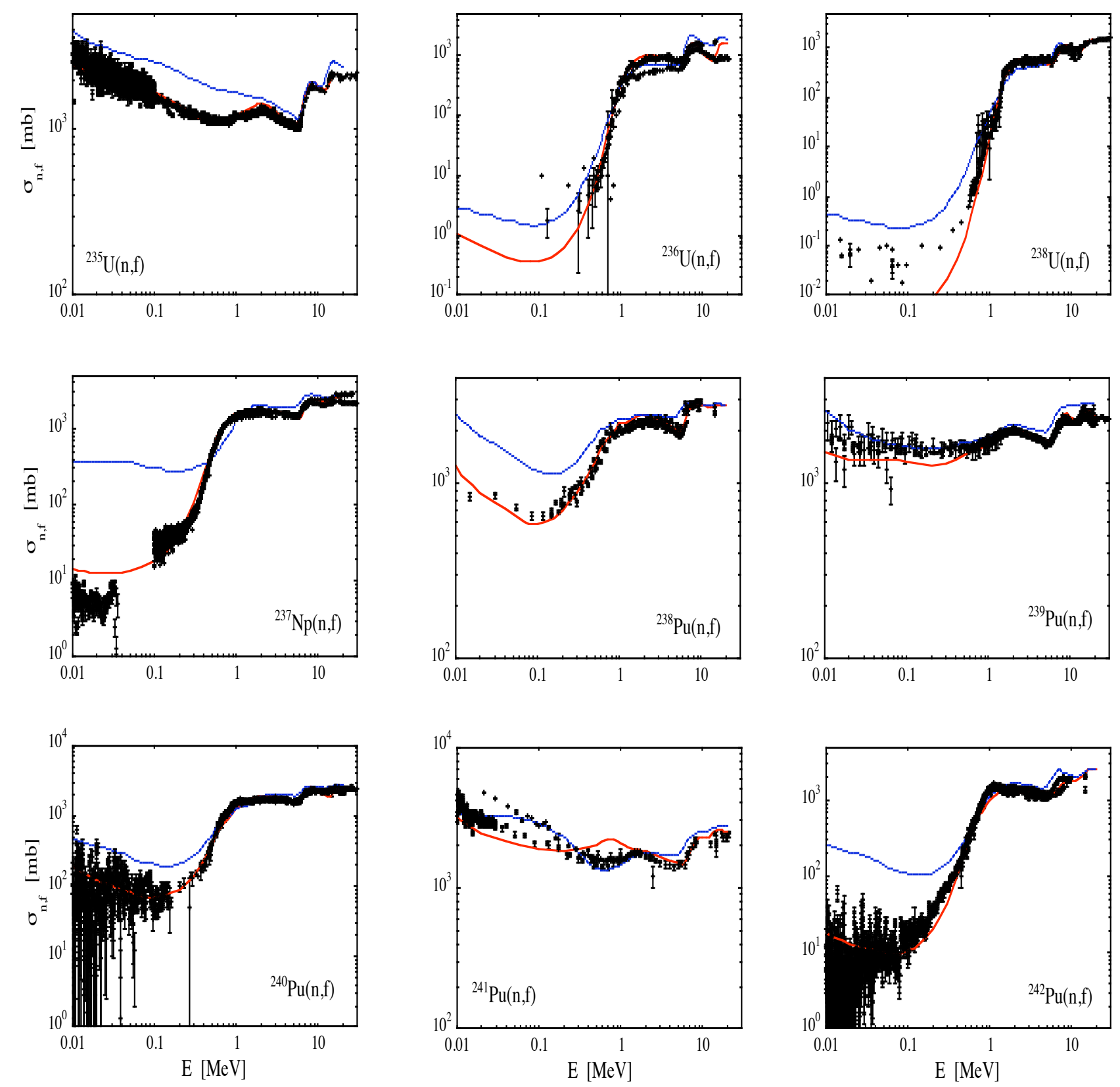

(1 unique set) 


\section{Fission still requires a lot of work}

- still not capable of predicting fission paths (barrier height at best 20\%) and NLD at saddle points

- very uncertain cross section for nuclei close to valley of $\beta$ stability

- even worse for $n$-induced, spontaneous and $\beta$-delayed fission rates as well as fission fragment distribution for exotic n-rich nuclei up to the n-drip line 


\section{Conclusions}

Nucleosynthesis applications involving exotic n- or p-rich nuclei is still characterized by many more open questions than answers

- astrophysics site: astrophysics problem, specific modeling (explosion hydrodynamics, EoS, v-physics, ...)

- nuclear physics of exotic nuclei: it is the role of nuclear physicist to provide the best possible nuclear ingredients based on Accurate, global and as microscopic as possible models

- nuclear reaction mechanisms (Equilibrium, Pre-Eq., DC)

- nuclear $\beta$-decay rates (Allowed \& Forbidden Transition for spherical but also deformed nuclei)

- nuclear structure properties (masses, deformation, densities,...)

- interaction (strong, weak, electromagnetic) properties: still many open questions when dealing with exotic n-rich nuclei

A lot of Theoretical as well as Experimental works still needed 